Final Report

FHWA/IN/JTRP-2001/11

\title{
Design Guidelines for Deploying Closed Loop Systems
}

\author{
By \\ Andrew Nichols \\ Graduate Research Assistant \\ Darcy Bullock \\ Associate Professor \\ School of Civil Engineering \\ Purdue University \\ Joint Transportation Research Program \\ Project No: C-36- 17YY \\ File No: 8-4-51 \\ SPR- 2390 \\ Conducted in Cooperation with the \\ Indiana Department of Transportation \\ and the \\ U.S. Department of Transportation \\ Federal Highway Administration
}

The contents of this report reflect the views of the authors, who are responsible for the facts and the accuracy of the data presented herein. The contents do not necessarily reflect the official views or policies of the Indiana Department of Transportation or the Federal Highway Administration at the time of publication. The report does not constitute a standard, specification, or regulation.

\author{
Purdue University \\ West Lafayette, IN 47907 \\ November 2001
}




\begin{tabular}{|c|c|c|}
\hline $\begin{array}{l}\text { 1. Report No. } \\
\text { FHWA/IN/JTRP-2001/11 }\end{array}$ & 2. Government Accession No. & 3. Recipient's Catalog No. \\
\hline \multirow{2}{*}{\multicolumn{2}{|c|}{$\begin{array}{l}\text { 4. Title and Subtitle } \\
\text { Design Guidelines for Deploying Closed Loop Systems }\end{array}$}} & $\begin{array}{l}\text { 5. } \quad \text { Report Date } \\
\text { November } 2001\end{array}$ \\
\hline & & 6. Performing Organization Code \\
\hline \multicolumn{2}{|l|}{$\begin{array}{l}\text { 7. Author(s) } \\
\text { Andrew Nichols and Darcy Bullock }\end{array}$} & $\begin{array}{l}\text { 8. Performing Organization Report No. } \\
\text { FHWA/IN/JTRP-2001/11 }\end{array}$ \\
\hline \multirow{2}{*}{\multicolumn{2}{|c|}{$\begin{array}{l}\text { 9. Performing Organization Name and Address } \\
\text { Joint Transportation Research Program } \\
1284 \text { Civil Engineering Building } \\
\text { Purdue University } \\
\text { West Lafayette, Indiana } 47907-1284\end{array}$}} & 10. Work Unit No. \\
\hline & & $\begin{array}{l}\text { 11. Contract or Grant No. } \\
\text { SPR- } 2390\end{array}$ \\
\hline \multirow{2}{*}{\multicolumn{2}{|c|}{$\begin{array}{l}\text { 12. Sponsoring Agency Name and Address } \\
\text { Indiana Department of Transportation } \\
\text { State Office Building } \\
100 \text { North Senate Avenue } \\
\text { Indianapolis. IN } 46204\end{array}$}} & $\begin{array}{c}\text { 13. Type of Report and Period Covered } \\
\text { Final Report }\end{array}$ \\
\hline & & 14. Sponsoring Agency Code \\
\hline
\end{tabular}

\section{Supplementary Notes}

Prepared in cooperation with the Indiana Department of Transportation and Federal Highway Administration.

\section{Abstract}

Closed loop systems are becoming the preferred method of operating coordinated traffic signal systems. Some of the benefits of a closed loop system include remote controller access, synchronization of controller time clocks, and orderly transition between coordination plans. With ensured time clock synchronization, the coordination of traffic signals becomes more reliable, which leads to optimal vehicle progression through the system. However, due to the complexity and relative infrequent implementation in more rural districts of closed loop systems, it is very difficult to obtain a successful deployment. This manual provides a step-by-step procedure for designing the parameters, implementing, testing, and field tuning closed loop systems. The procedure is described for an example closed loop system consisting of five intersections in West Lafayette, Indiana.

The guidelines for the design of signal timing parameters will serve as a manual for all Indiana Department of Transportation districts. Detailed configuration procedures are provided for Econlite and Peek controllers, as well as their respective management software packages. Also included in the implementation procedure are instructions for interconnecting the controllers and establishing communication between the controllers. The testing procedure described in this manual is a hardware-in-the-loop simulation, which uses a controller interface device (CID) in conjunction with CORSIM software. Field tuning practices are documented for detectors, timing parameters, and coordination plans.

\section{Key Words}

Traffic signal controller, closed loop system, signal timing calculations, hardware-in-the-loop simulation, field tuning, implementation.

\section{Distribution Statement}

No restrictions. This document is available to the public through the National Technical Information Service, Springfield, VA 22161

\begin{tabular}{|c|c|c|c|}
\hline $\begin{array}{c}\text { 19. Security Classif. (of this report) } \\
\text { Unclassified }\end{array}$ & $\begin{array}{c}\text { 20. Security Classif. (of this page) } \\
\text { Unclassified }\end{array}$ & 22. Price \\
\hline
\end{tabular}




\section{TECHNICAL Summary}

INDOT Research

Technology Transfer and Project Implementation Information

TRB Subject Code: 54-5 Traffic Control Devices

Publication No.: FHWA/IN/JTRP-2001/11, SPR-2390

November 2001

Final Report

\section{Design Guidelines for Deploying Closed Loop Systems}

\section{Introduction}

There are over 200 traffic signal systems consisting of about 1,000 traffic signals that are owned and maintained by the Indiana Department of Transportation (INDOT). These closed loop systems are coordinated by interconnection or by time. With this many signals, INDOT is looking for ways to improve consistency and decrease the amount of time spent designing, implementing, and maintaining the signal controllers. Currently, the determination of system parameters is unique to each district and there are no guidelines or procedures to follow. An objective of drafting this manual is to establish a traffic signal control design procedure for all INDOT districts to follow for deploying closed loop signal systems. Currently, the determination of system parameters is unique to each district and there are no guidelines or procedures to follow. This manual documents in writing a synthesis of best practices currently used by various districts. The manual will also serve as an educational tool for new INDOT employees and consultants.

\section{Findings}

The first part of this manual documents the general process for determining parameters for traffic signals and traffic signal systems. The important aspects of the design were determined to be the timing parameters, detectors, time-ofday scheduling, and coordination for systems. The second part of this manual provides a detailed description of how to configure two vendors' traffic signal controllers to deploy a closed loop system. A closed loop system typically consists of a series of intersections that are interconnected and programmed to provide progression along an arterial. The final part of the manual deals with testing the controllers in a lab environment before they are implemented in the field and tuning them after they are implemented. The testing is accomplished by performing a hardware-in-the-loop simulation using a controller interface device and a simulation program such as CORSIM. Tuning decisions can be made by looking at the performance of the hardware-in-the-loop simulation or by viewing the real time performance.

\section{Implementation}

This manual will serve as a reference for those who are experienced in the traffic signal field and as a training tool for those who have never programmed controllers. Employee turnover rates are very high in government work with frequent promotions and transfers. Instead of a current engineer teaching the new employee, this manual will guide them through the process and the engineer can supplement the manual with any information specific to that district. This tool will help facilitate the deployment of new systems so that more time can be spent improving the performance of existing systems. 


\section{Contacts}

For more information:

Prof. Darcy Bullock

Principal Investigator

School of Civil Engineering

Purdue University

West Lafayette IN 47907

Phone: (765) 494-2226

Fax: (765) 496-1105
Indiana Department of Transportation

Division of Research

1205 Montgomery Street

P.O. Box 2279

West Lafayette, IN 47906

Phone: (765) 463-1521

Fax: (765) 497-1665

\section{Purdue University}

Joint Transportation Research Program

School of Civil Engineering

West Lafayette, IN 47907-1284

Phone: (765) 494-9310

Fax: (765) 496-1105 


\section{Table of Contents}

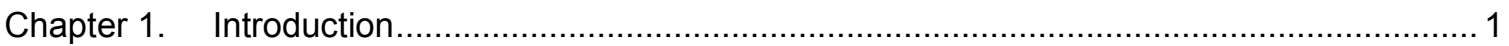

Chapter 2. Closed Loop System Design Procedures …..................................................... 4

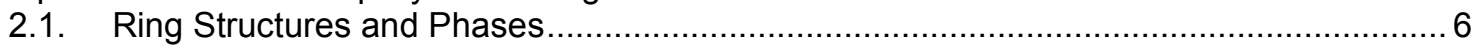

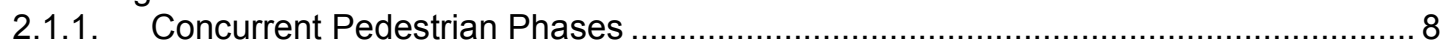

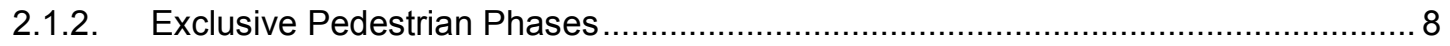

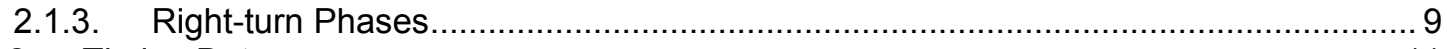

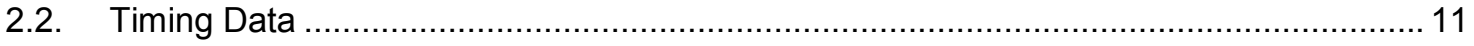

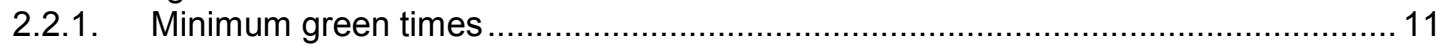

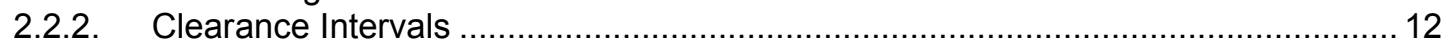

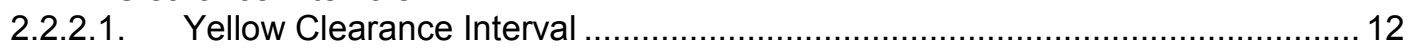

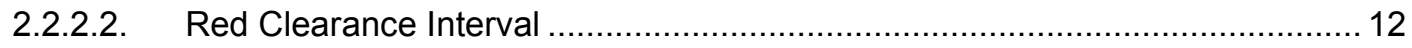

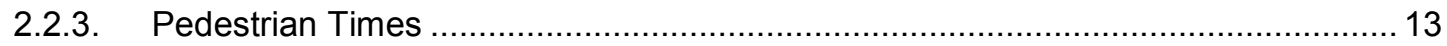

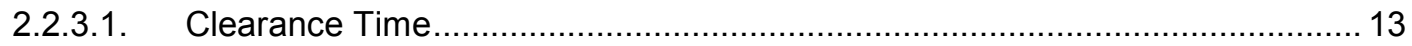

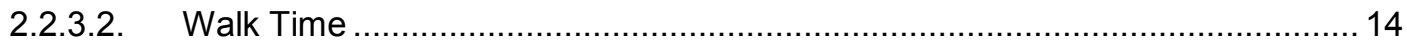

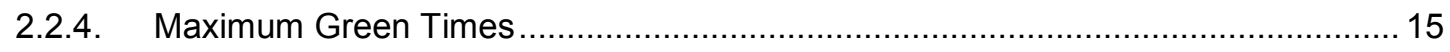

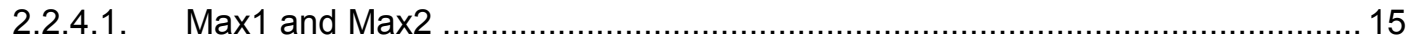

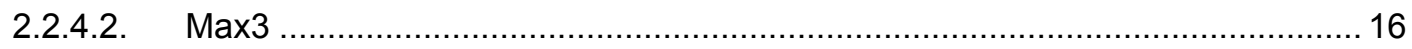

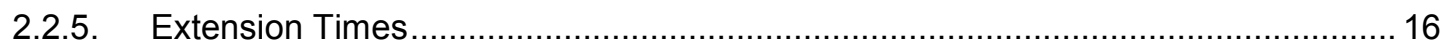

2.2.5.1. Vehicle Extension for Stopbar Detection .................................................... 16

2.2.5.2. Vehicle Extension for Advance Detection ..................................................... 18

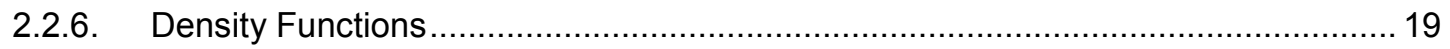

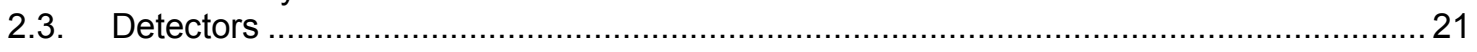

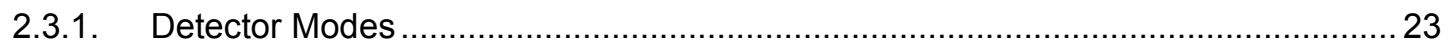

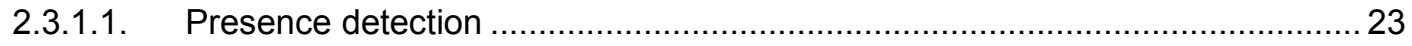

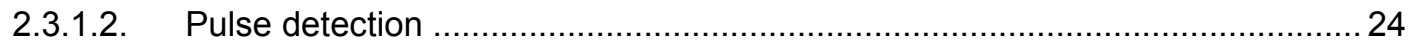

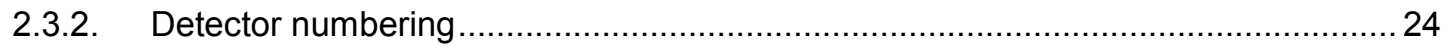

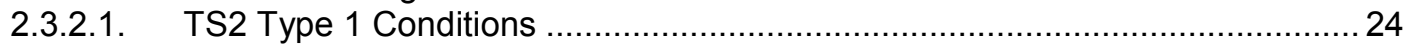

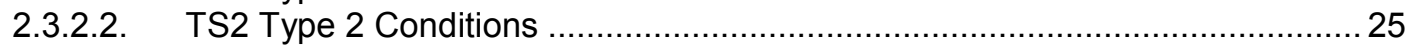

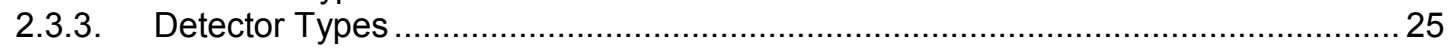

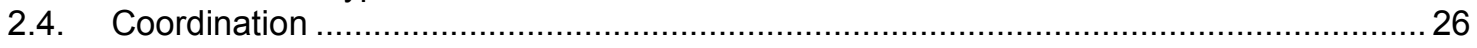

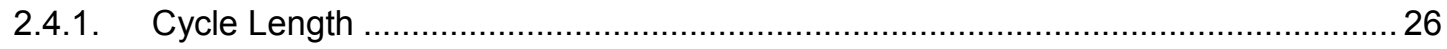

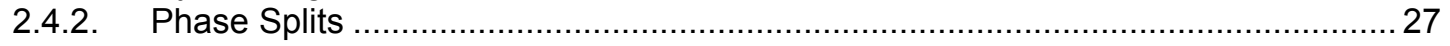

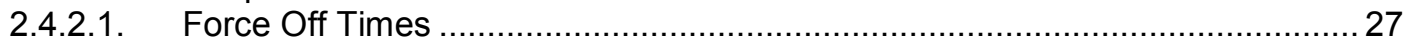

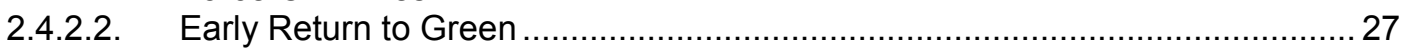

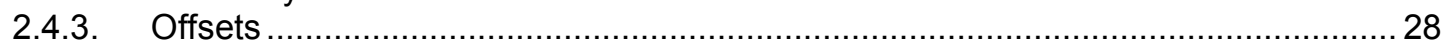

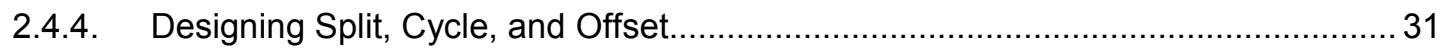

Chapter 3. Configuring Econolite Closed Loop Traffic Signal Systems .................................... 32

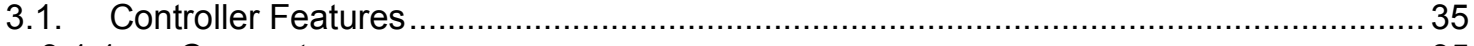

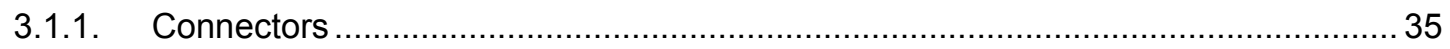

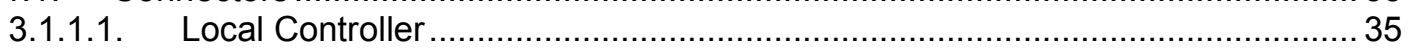

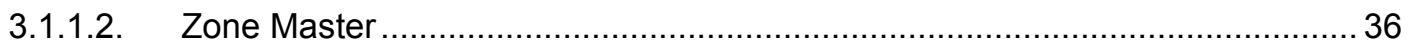

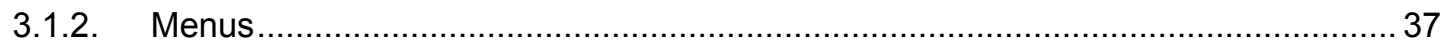

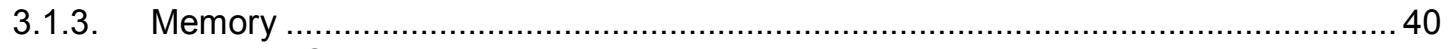

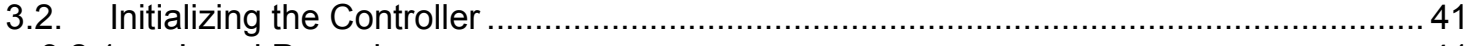

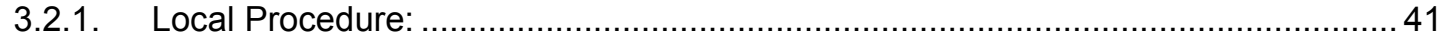

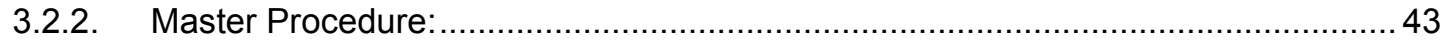

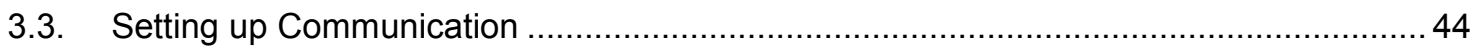

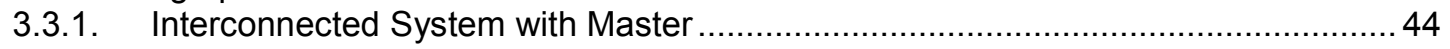

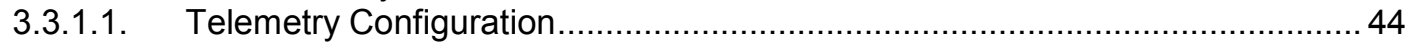

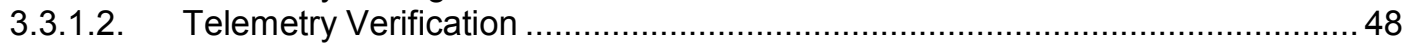

3.3.1.3. Interconnected System Time Clock .............................................................. 49

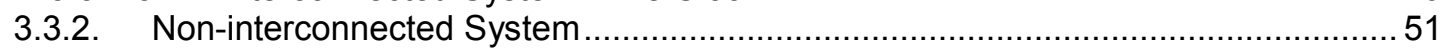




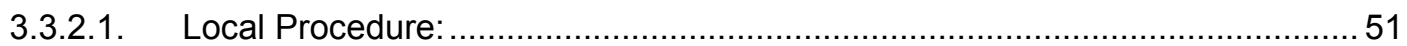

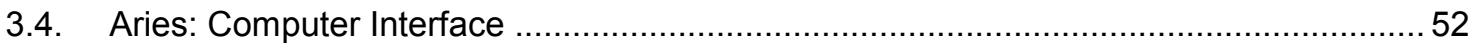

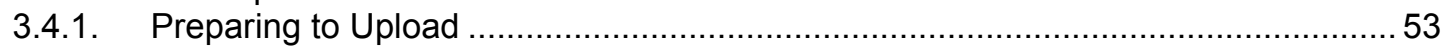

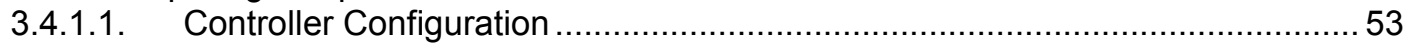

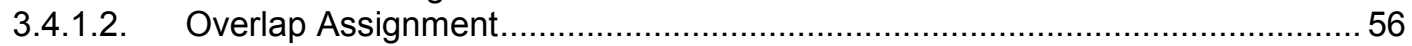

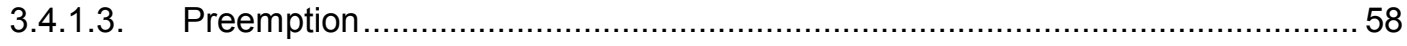

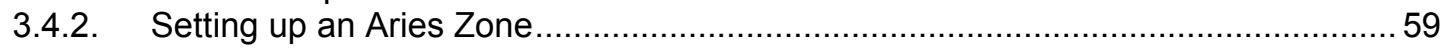

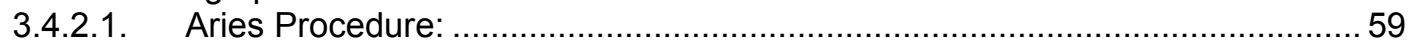

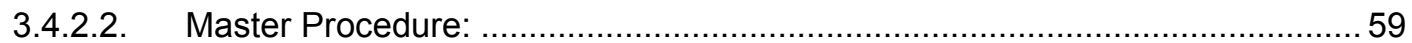

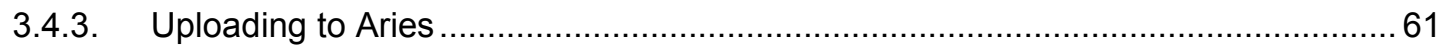

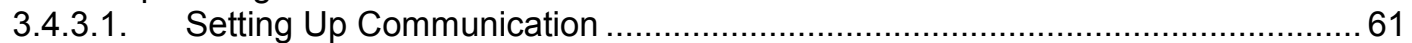

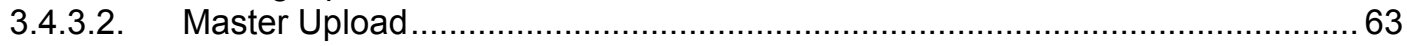

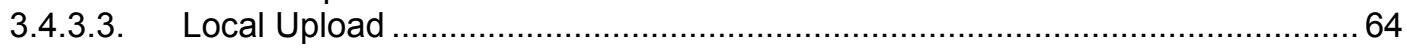

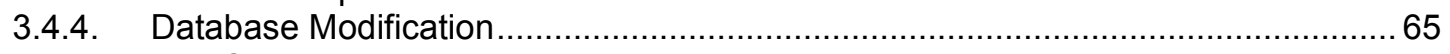

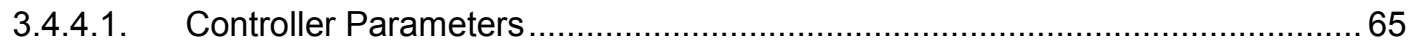

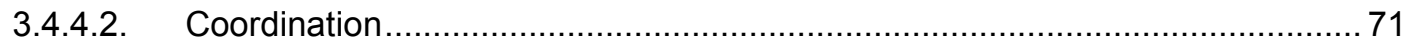

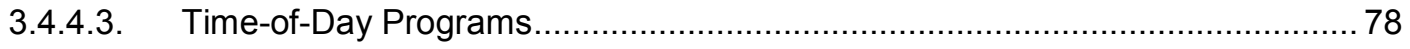

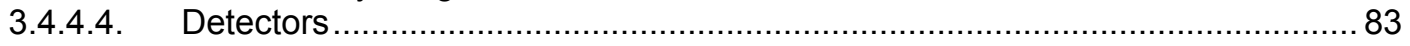

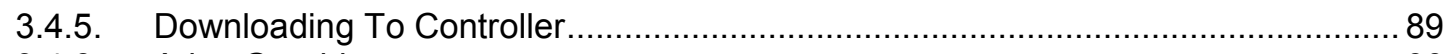

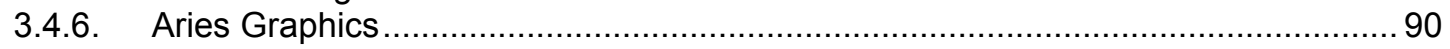

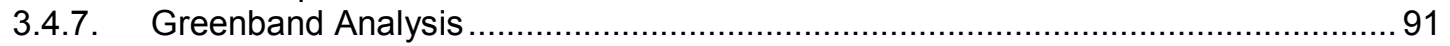

Chapter 4. Configuring Peek Closed Loop Traffic Signal Systems ........................................ 93

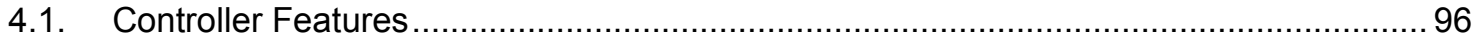

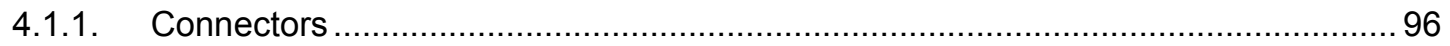

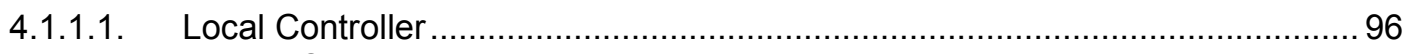

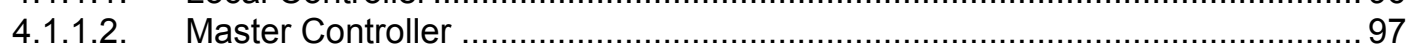

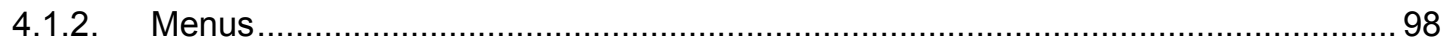

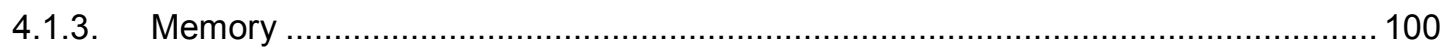

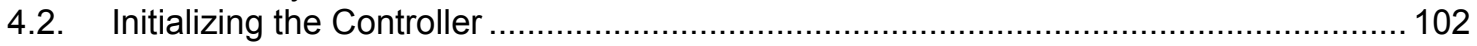

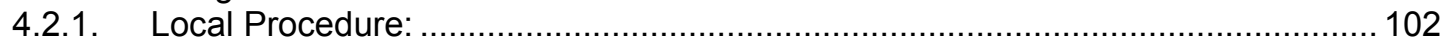

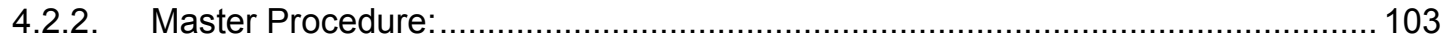

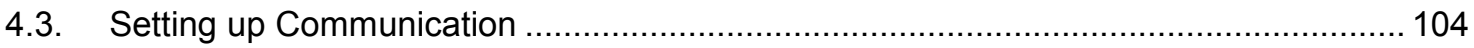

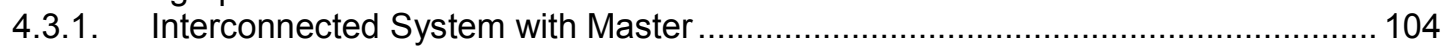

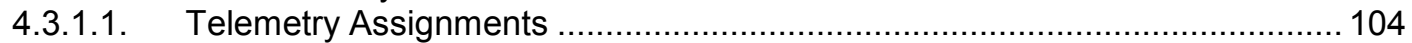

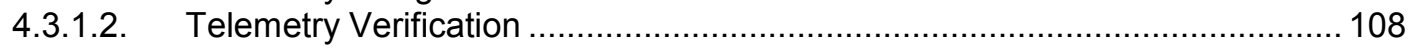

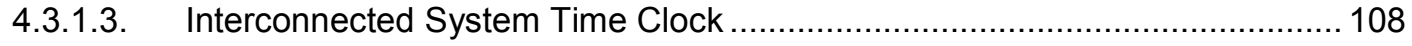

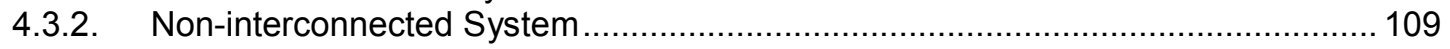

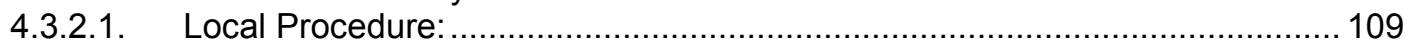

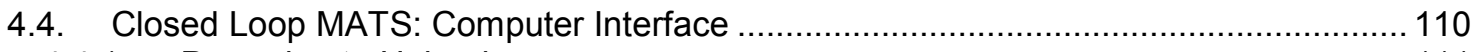

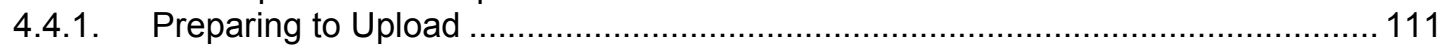

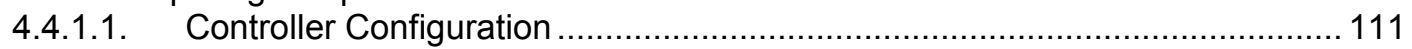

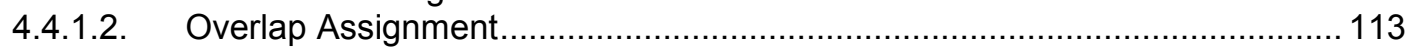

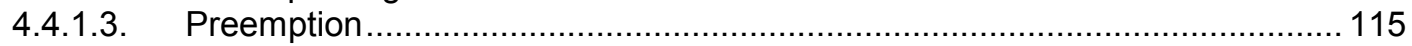

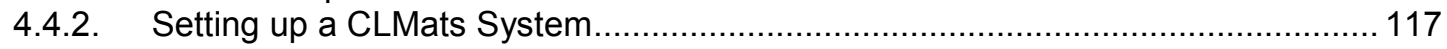

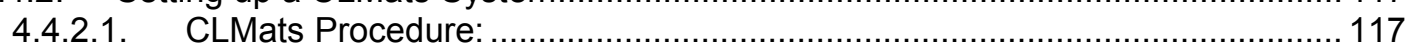

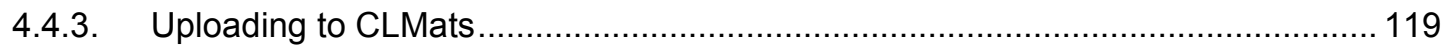

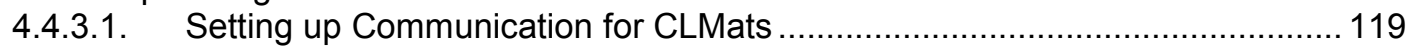

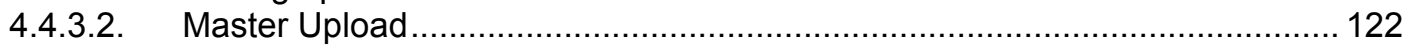

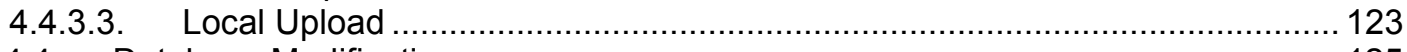

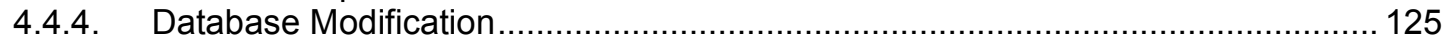

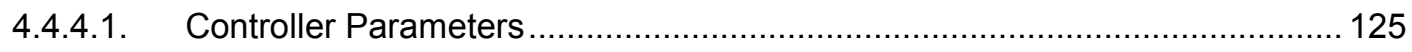

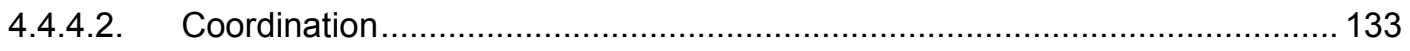

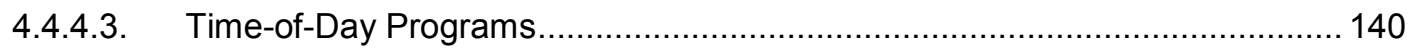

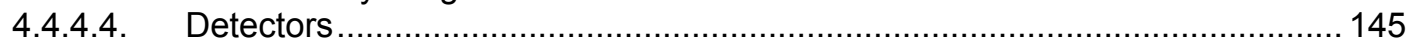

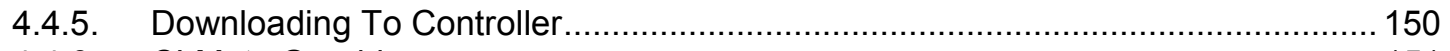

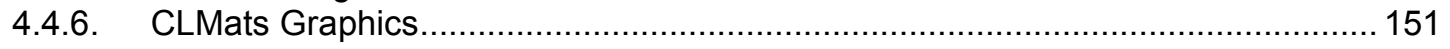




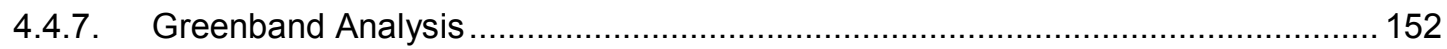

Chapter 5. Hardware-in-the-loop Simulation for Testing Controllers ................................... 153

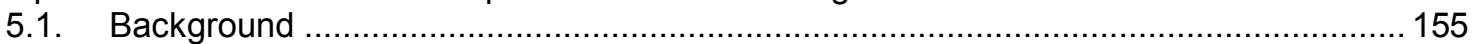

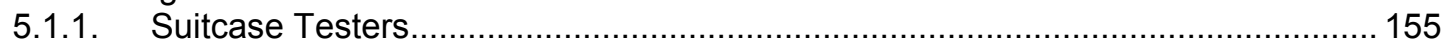

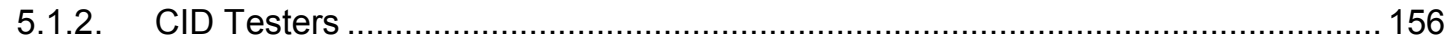

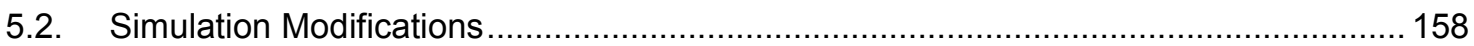

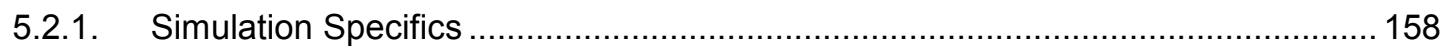

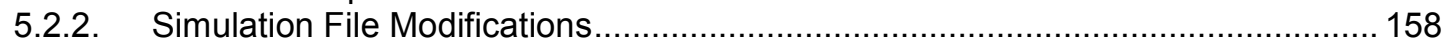

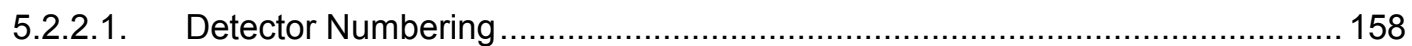

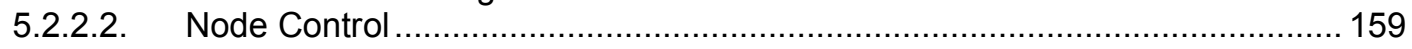

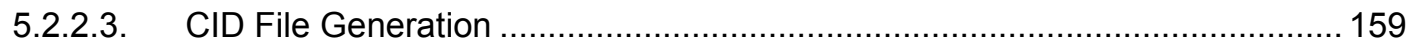

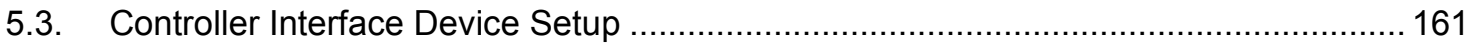

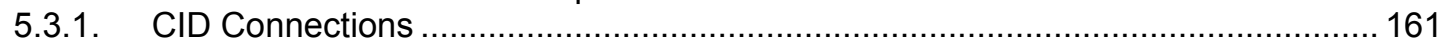

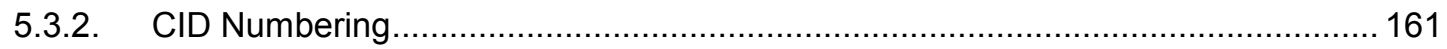

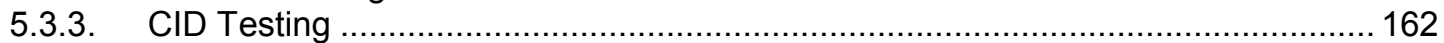

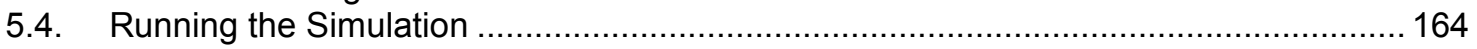

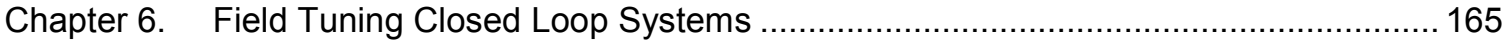

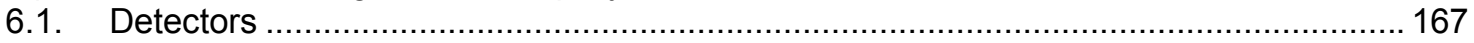

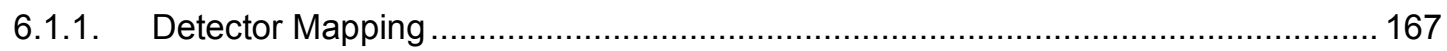

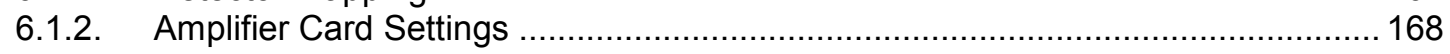

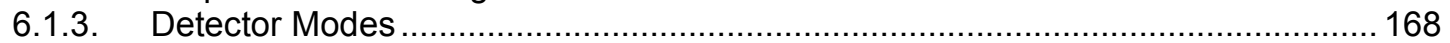

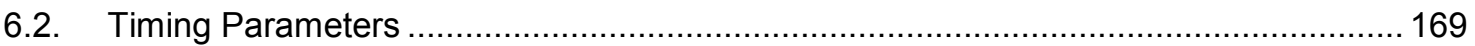

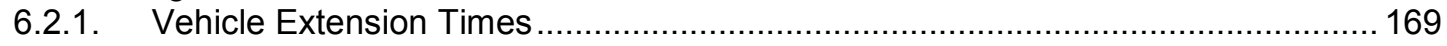

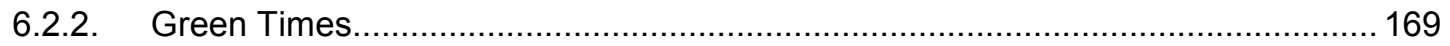

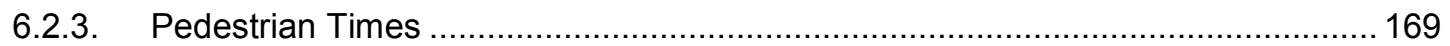

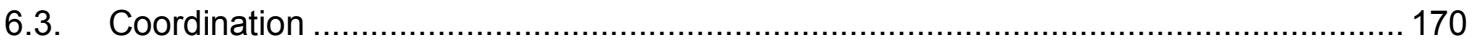

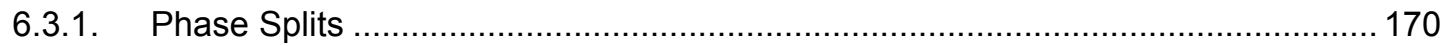

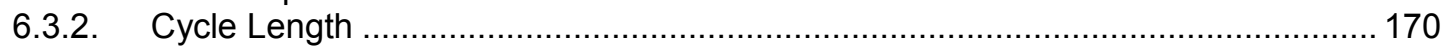

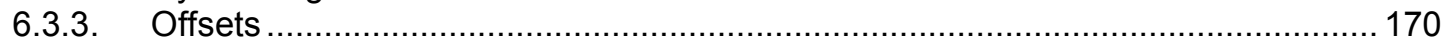

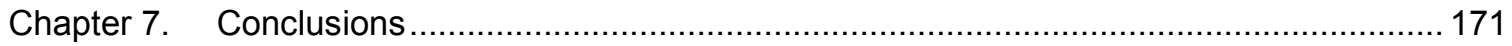

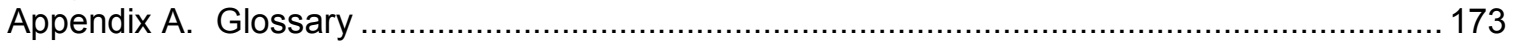

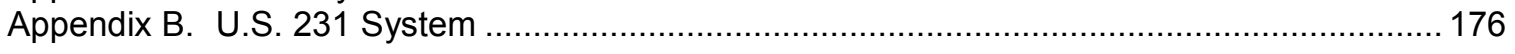




\section{List of Tables}

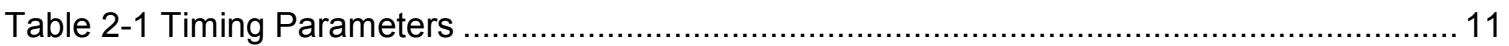

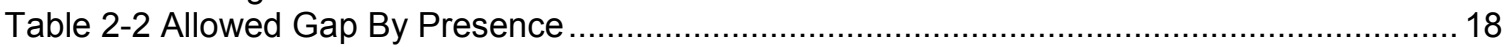

Table 2-3 Maximum Detector Setback (ft) for Various Speeds and Gap Extension Times .......... 19

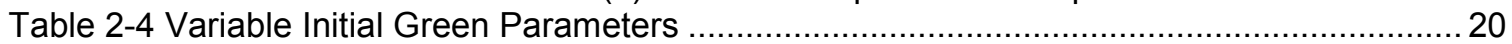

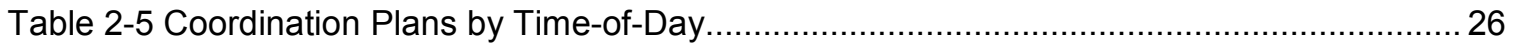

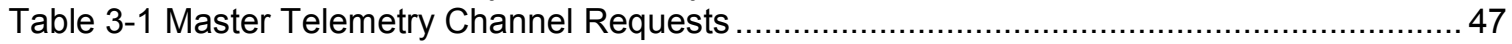

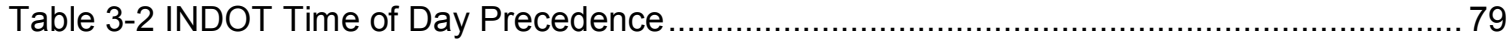

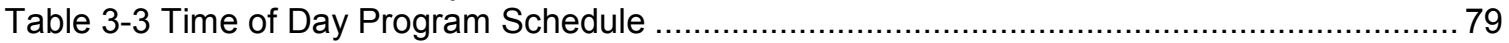

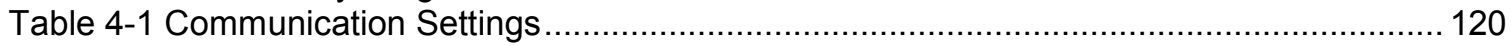

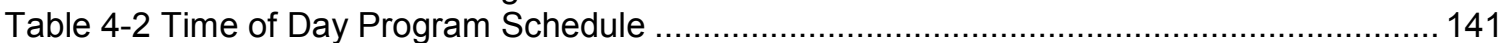

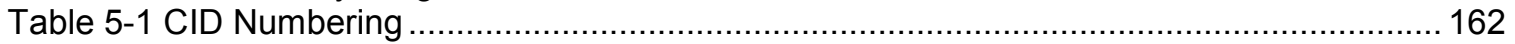

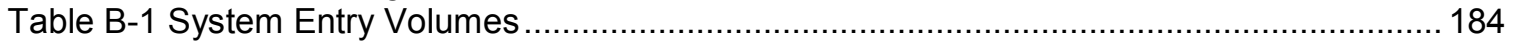

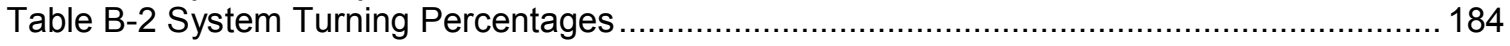

Table B-3 Intersection Pocket Lengths and Detector Locations................................................ 185

Table B-4 Controller Interface Device File (used for Hardware-in-the-Loop) ........................... 186

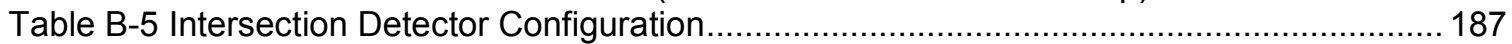

Table B-6 General Phase Parameters for Intersection 1 .................................................. 188

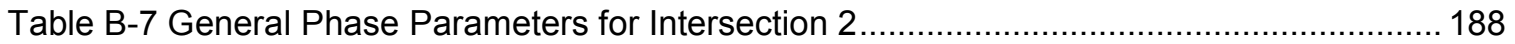

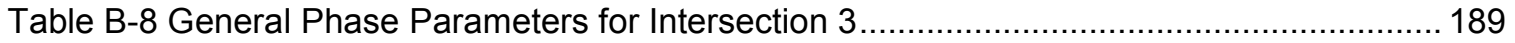

Table B-9 General Phase Parameters for Intersection 4 ................................................... 189

Table B-10 General Phase Parameters for Intersection 5 ................................................. 190

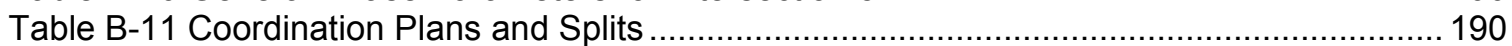




\section{List of Figures}

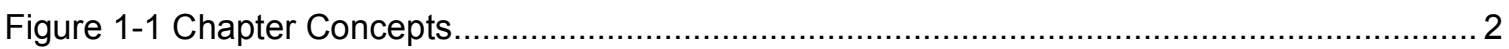

Figure 2-1 Chapter Concepts - Signal Timing Design Procedure ............................................ 5

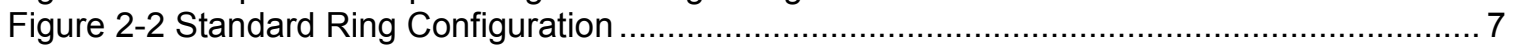

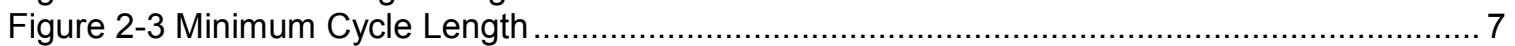

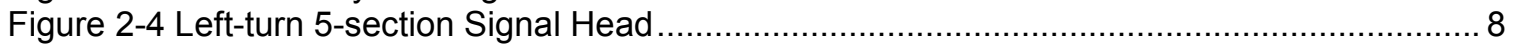

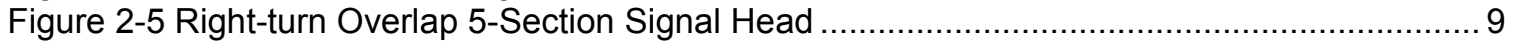

Figure 2-6 Standard Ring Configuration with Overlaps .............................................................. 10

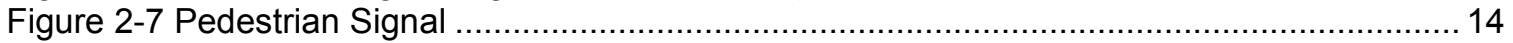

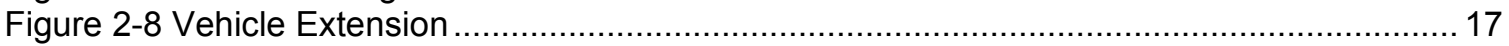

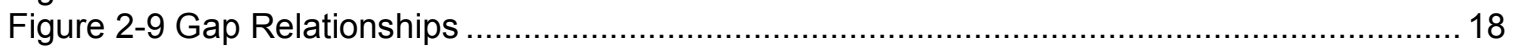

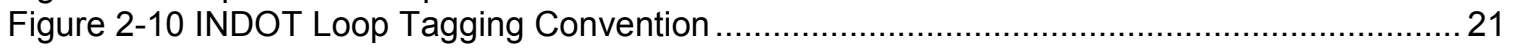

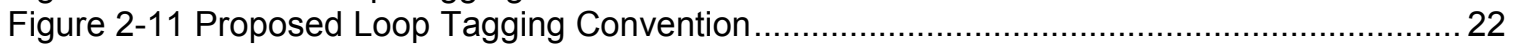

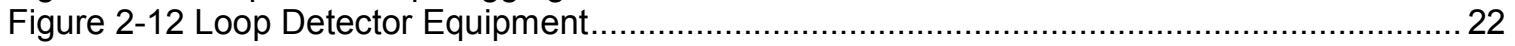

Figure 2-13 Four-legged Intersection with Four-Lane Approaches .......................................... 23

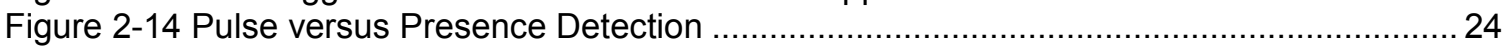

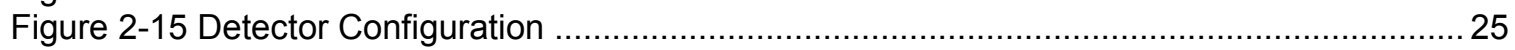

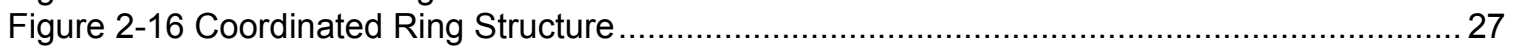

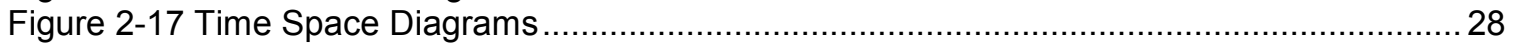

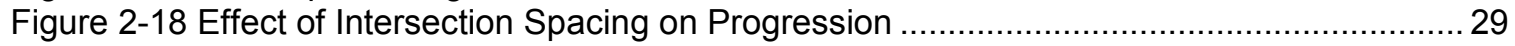

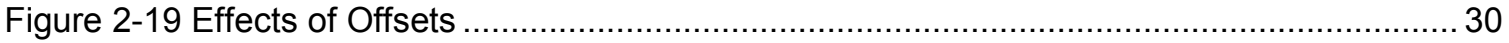

Figure 3-1 Chapter Concepts - Details of Configuring an Econolite System .............................. 33

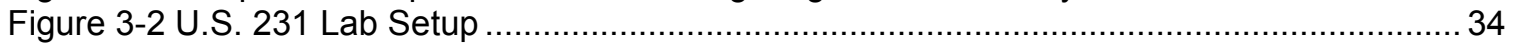

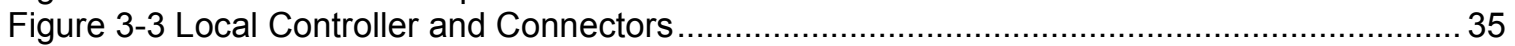

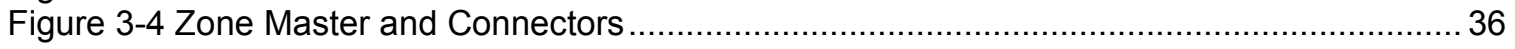

Figure 3-5 Econolite Local Controller Main Menu and Keyboard ................................................. 37

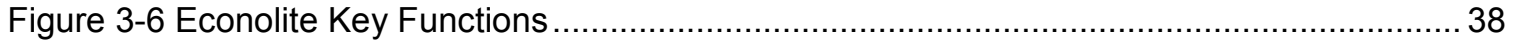

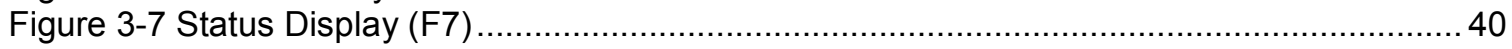

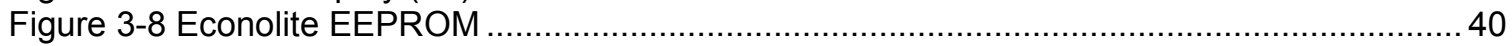

Figure 3-9 Initialization: Local Controller Copy Backup Timing ............................................... 42

Figure 3-10 Initialization: Local Controller Memory Clear....................................................... 42

Figure 3-11 Communication: Local Controller Port 2 Configuration (MM, 1, 5) ......................... 44

Figure 3-12 Communication: Local Controller Port 3 Configuration ............................................ 45

Figure 3-13 Communication: Master Telemetry Channel Assignments ...................................... 46

Figure 3-14 Communication: Master Telemetry Channel Configuration ................................... 47

Figure 3-15 Communication: Master Modem Configuration ................................................ 48

Figure 3-16 Communication: Master Telemetry Verification ................................................. 48

Figure 3-17 Communication: Local Telemetry Verification (F7) …....................................... 49

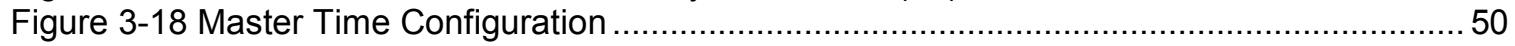

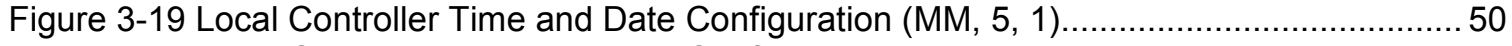

Figure 3-20 Local Controller Time and Date Configuration (MM, 5, 1)................................... 51

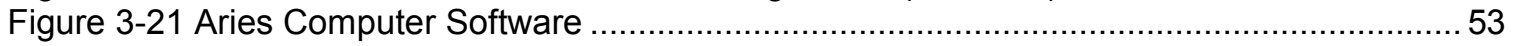

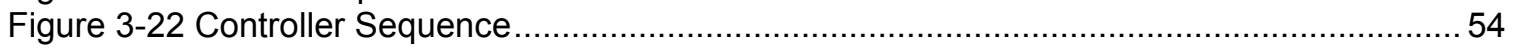

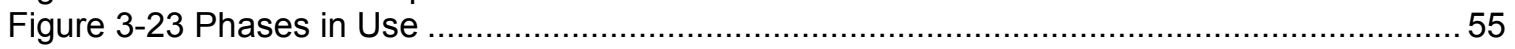

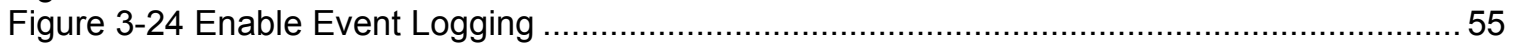

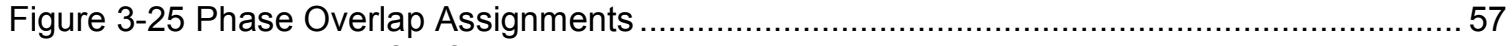

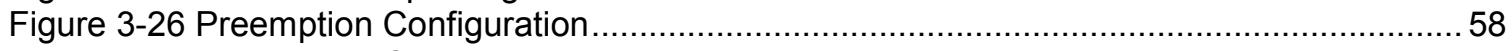

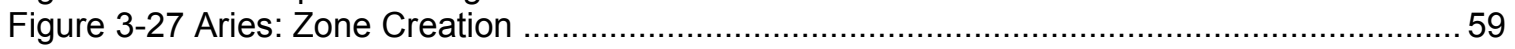

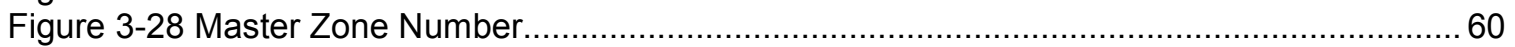

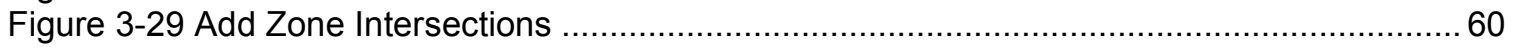

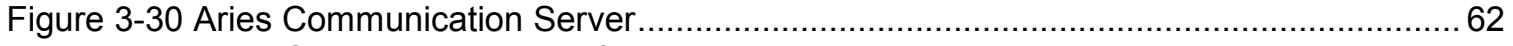

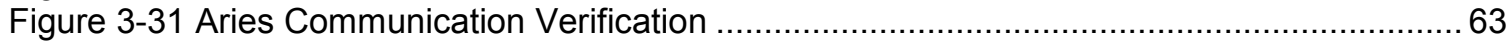

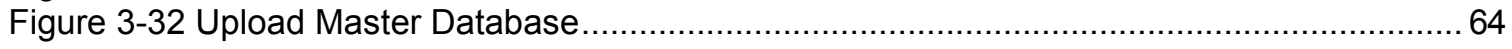




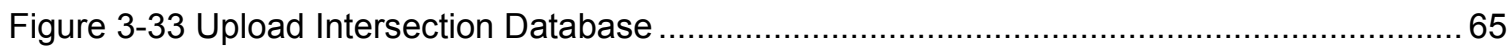

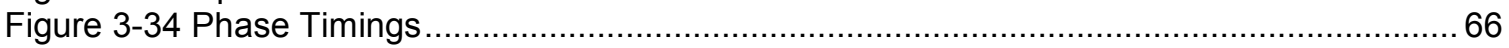

Figure 3-35 Phase Timing (Minimum, Pedestrian, Extension, Maximums).................................. 67

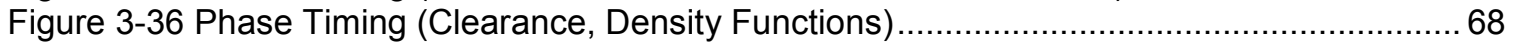

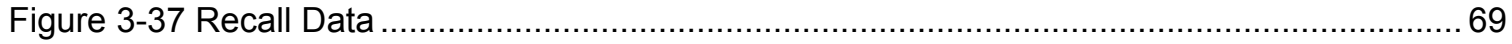

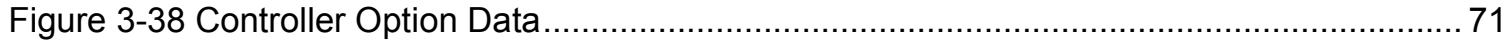

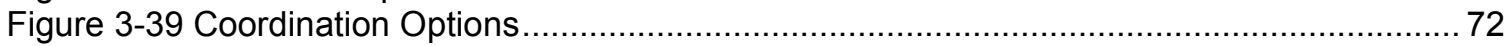

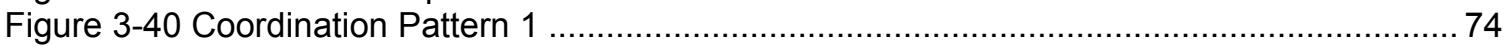

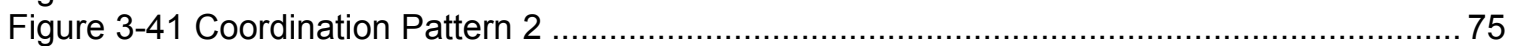

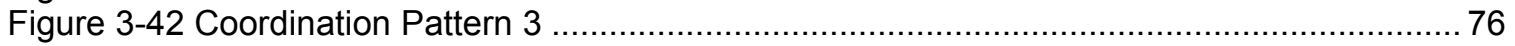

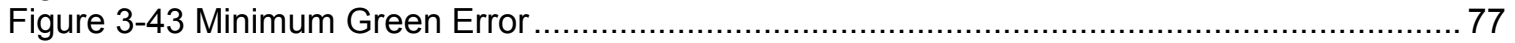

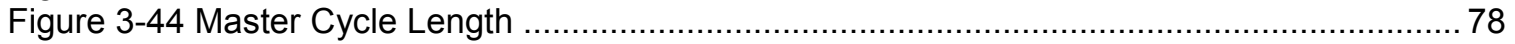

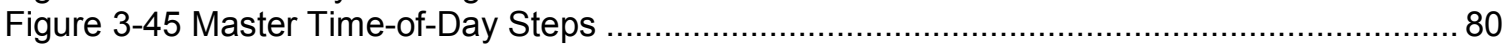

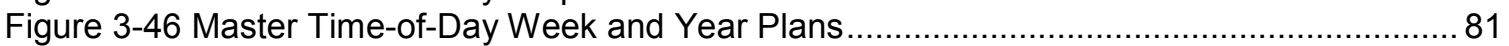

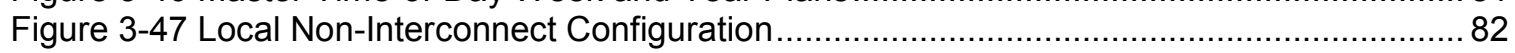

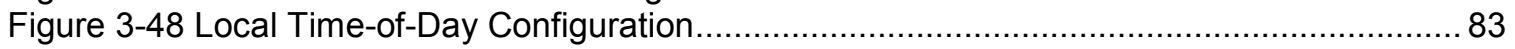

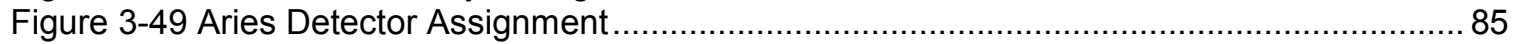

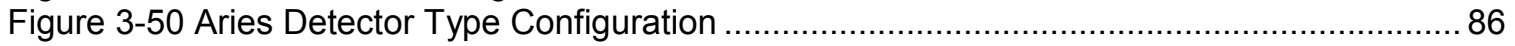

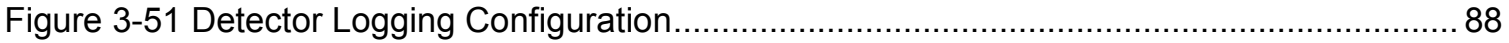

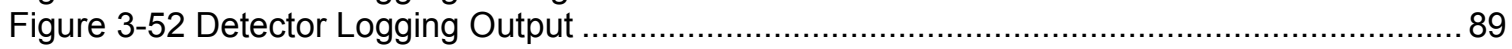

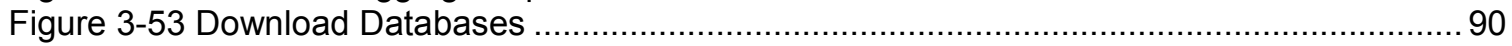

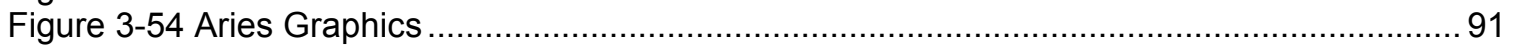

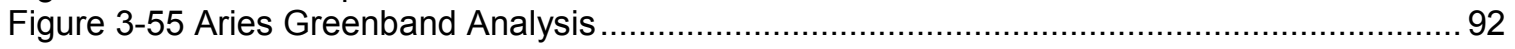

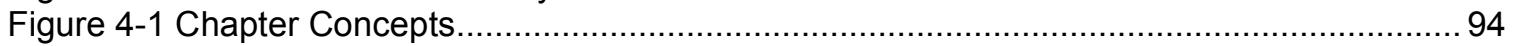

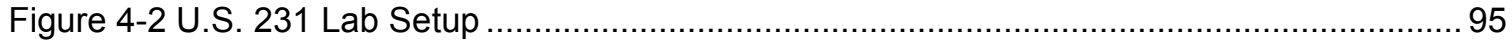

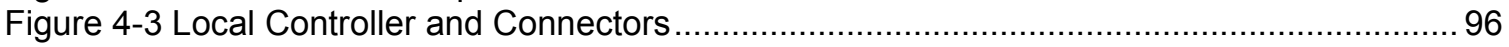

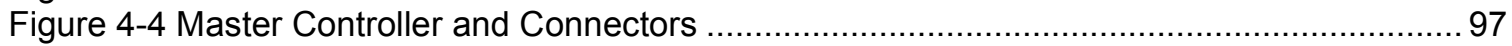

Figure 4-5 Peek Local Controller Main Menu and Keyboard...................................................... 98

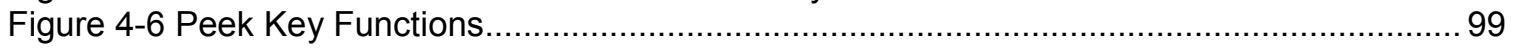

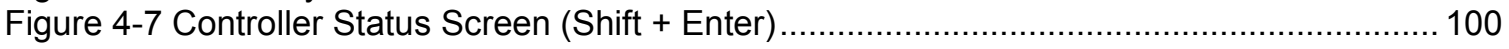

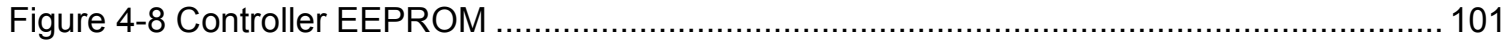

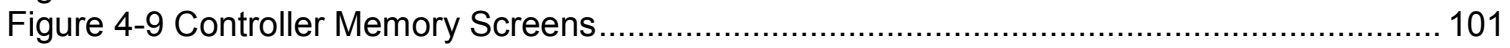

Figure 4-10 Initialization: Local Controller Default Data Load ............................................... 102

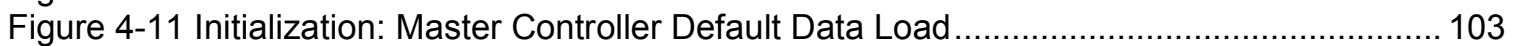

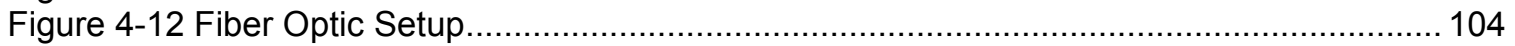

Figure 4-13 Communication: Local Controller Identification (MM, 3, 5, 5) ............................ 105

Figure 4-14 Communication: Local Controller Master Port Configuration (MM, 3, 5, 4, 3) ......... 105

Figure 4-15 Communication: Local Controller Port 2 Configuration (MM, 3, 5, 4, 3, PgDn) ....... 106

Figure 4-16 Communication: Local Controller Port 3 Configuration (MM, 3, 5, 4, 3, PgDn(2)) ... 106

Figure 4-17 Communication: Master Controller Identification Configuration (MM, 3, 1) ............ 106

Figure 4-18 Communication: Master Controller Port 2 Configuration (MM, 3, 8, 3) .................. 107

Figure 4-19 Communication: Master Controller Port 3 Configuration (MM, 3, 8, 3, PgDn) ......... 107

Figure 4-20 Communication: Master Local Enable (MM, 3, 2) .............................................. 107

Figure 4-21 Communication: Master Telemetry Verification (MM, 1, 1, 2) ................................ 108

Figure 4-22 Master Time and Date Configuration (MM, 3, 3, 8) ......................................... 109

Figure 4-23 Local Controller Time and Date Configuration (MM, 3, 3, 9) ............................... 109

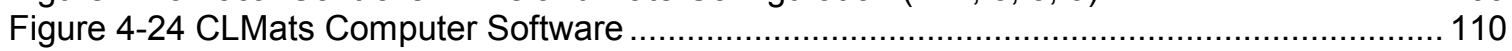

Figure 4-25 Controller Sequence Configuration ................................................................ 112

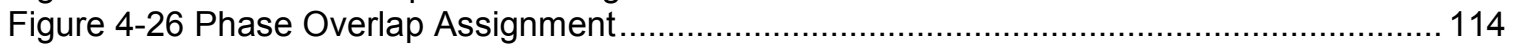

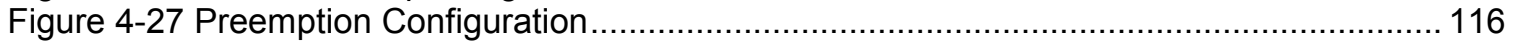

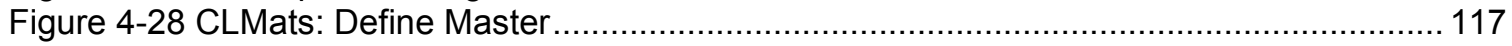

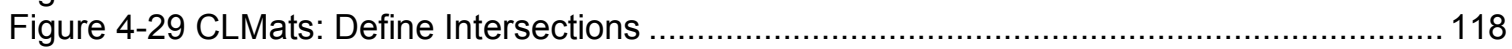

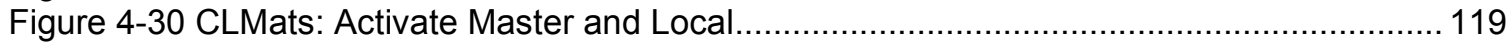

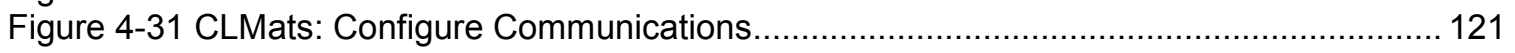

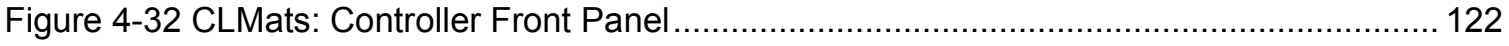

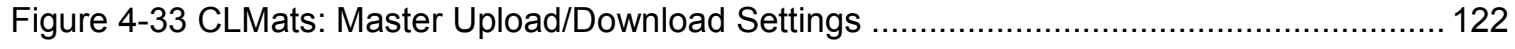




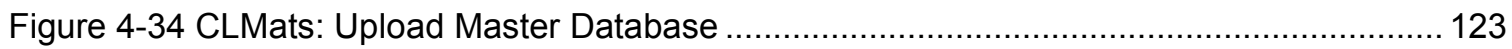

Figure 4-35 CLMats: Local Upload/Download Settings ..................................................... 124

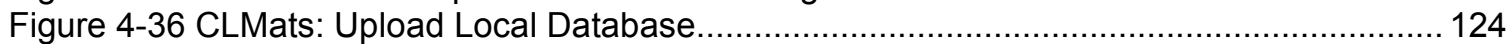

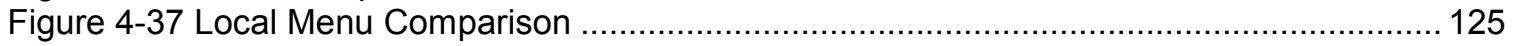

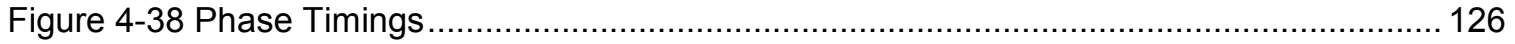

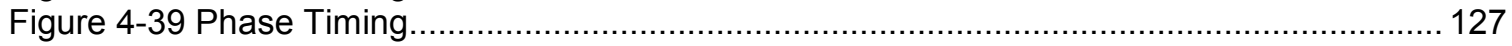

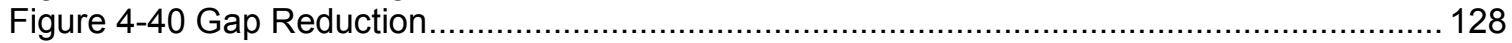

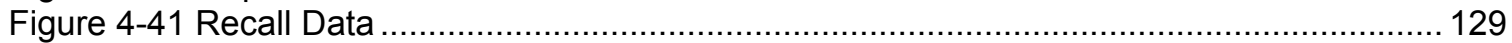

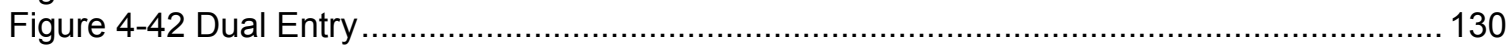

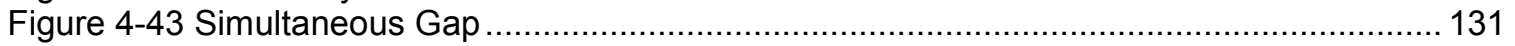

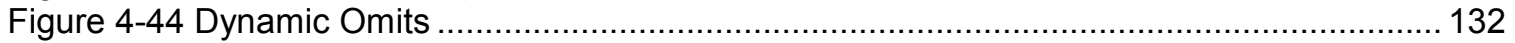

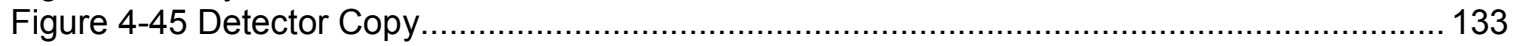

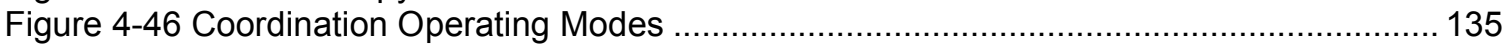

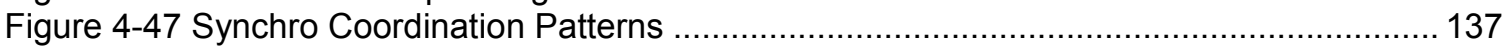

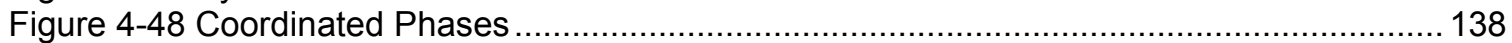

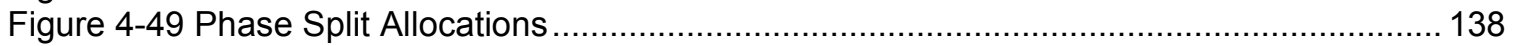

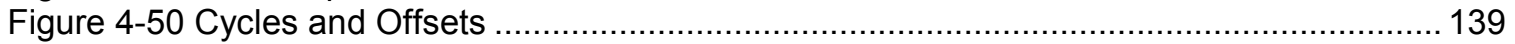

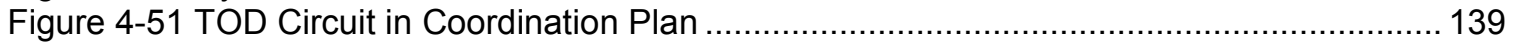

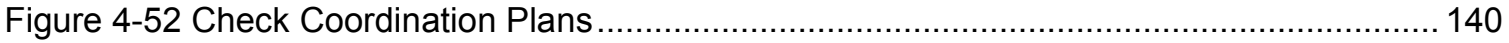

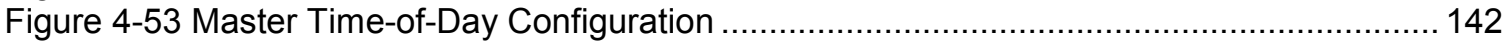

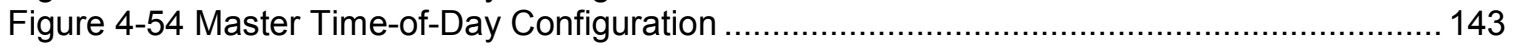

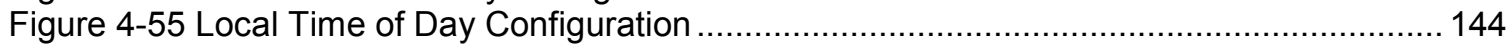

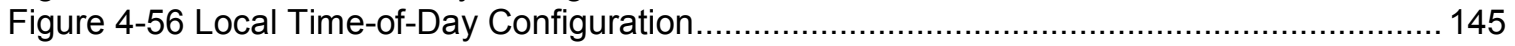

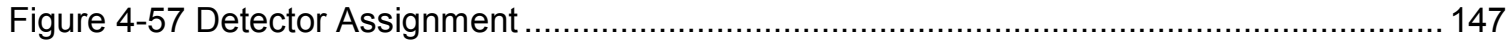

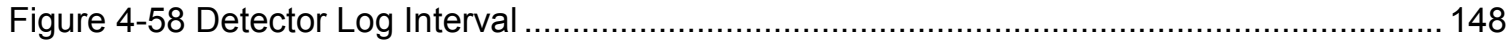

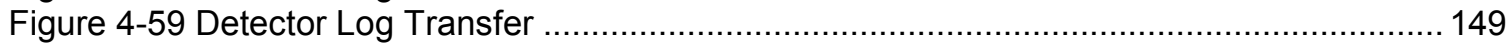

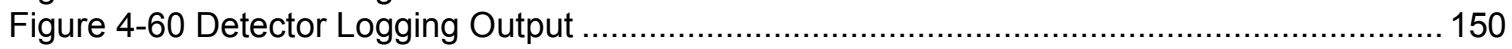

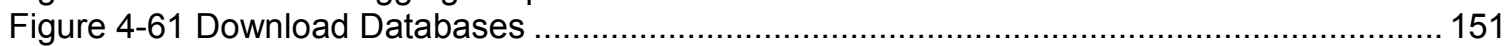

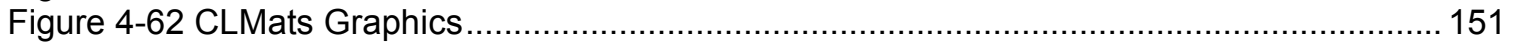

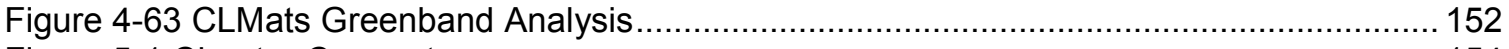

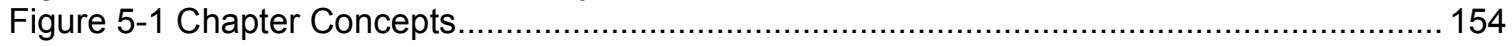

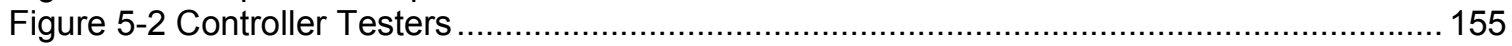

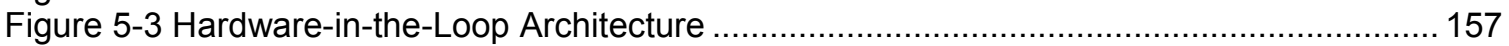

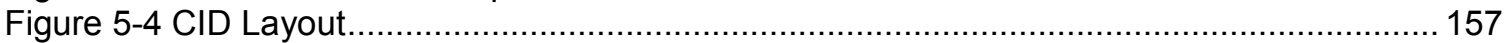

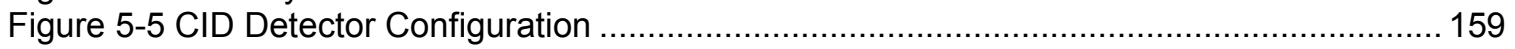

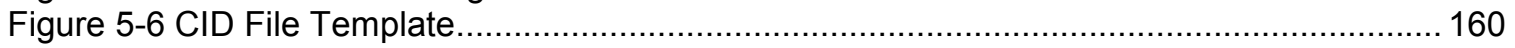

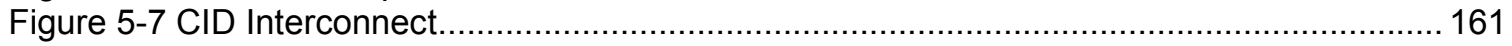

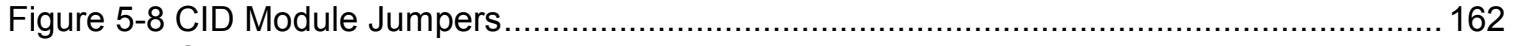

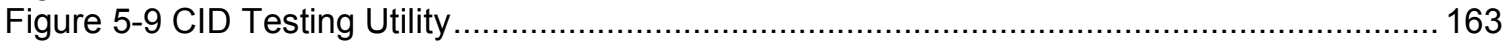

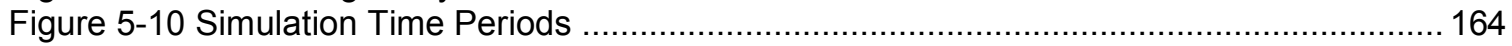

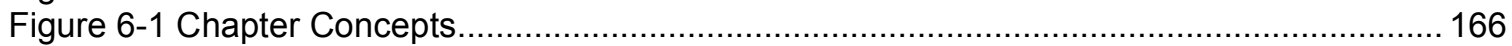

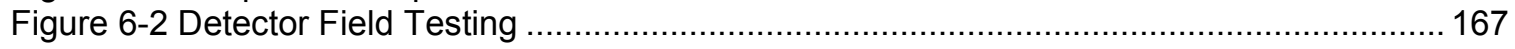

Figure B-1 US 231 (River Road) Network - State St. to Robinson St...................................177

Figure B-2 US 231 (River Road) Network - State St. to Robinson St. (Screen Capture) ............ 178

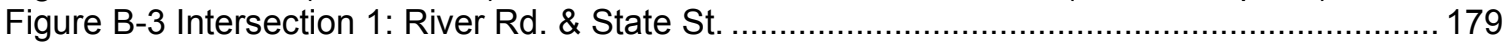

Figure B-4 Intersection 2: River Rd. \& Howard Ave. ....................................................... 180

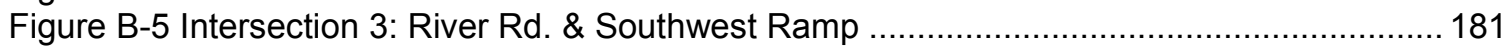

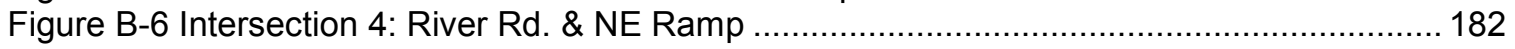

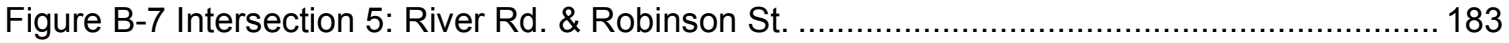




\section{Implementation Report}

There are over 200 traffic signal systems consisting of about 1,000 traffic signals that are owned and maintained by the Indiana Department of Transportation (INDOT). These closed loop systems are coordinated by interconnection or by time. With this many signals, INDOT is looking for ways to improve consistency and decrease the amount of time spent designing, implementing, and maintaining the signal controllers. Currently, the determination of system parameters is unique to each district and there are no guidelines or procedures to follow. An objective of drafting this manual is to establish a traffic signal control design procedure for all INDOT districts to follow for deploying closed loop signal systems. This manual documents in writing a synthesis of best practices currently used by various districts. The manual will also serve as an educational tool for new INDOT employees and consultants.

The first part of this manual documents the general process for determining parameters for traffic signals and traffic signal systems. The important aspects of the design were determined to be the timing parameters, detectors, time-of-day scheduling, and coordination for systems. Design standards are established for obtaining the timing parameters, based on intersection geometry and other published data. A standard detector numbering system is included, along with how the different detector settings are used. Recommendations are provided on the number of different signal timing plans for which design volumes are required and the approximate schedule for running those plans. Detailed information is provided on signal timing plans for coordination.

The second part of this manual provides a detailed description of how to configure two vendors' traffic signal controllers to deploy a closed loop system. A closed loop system typically consists of a series of intersections that are interconnected and programmed to provide progression along an arterial. A closed loop system can also be monitored remotely with management software over a telephone line. Every aspect of the configuration is covered that includes controller initialization, interconnection, data entry, and remote software setup. The two chapters follow the same sequence for configuring the system, so that one vendor's procedure can easily be compared to the other.

The final part of the manual deals with testing the controllers before they are implemented in the field and tuning them after they are implemented. The controllers are tested using a hardware-inthe-loop simulation. Details specific to setting up the simulation and creating necessary files are discussed. Finally, recommendations are provided for tuning the controllers after they are installed in the field. 


\section{Acknowledgments}

This document is a synthesis of knowledge obtained from the Study Advisory Committee, vendors, and external reviewers. Ryan Gallagher and Jim Sturdevant devoted considerable time assisting us in documenting current INDOT practices. Econolite Control Corporation, Traffic Control Corporation, Peek Corporation and the Traffic Signal Company supplied equipment used for testing the concepts provided in this document. In addition, Mike Shea (Econolite Control Corp), Mike Parks (Traffic Signal Company), Mike Boydstun (Ada County Highway Department, ID) and Carl Tuttle (INDOT), provided detailed review comments that assisted us in correcting errors and clarifying technical content. 


\section{Chapter 1. Introduction}

Traffic signal timing design is a relatively complex task requiring clear documentation of clearance times, pedestrian times, detector placement, detector operation, coordination schedules, and coordination plans. If these parameters are not carefully defined and implemented, effective closed loop operation cannot be obtained ${ }^{1}$.

Coordination of traffic signals is designed to allow smooth progression for traffic through a system, whether it is for an arterial, diamond interchange, or downtown district. A coordination plan has three main parameters: the cycle length, the phase splits, and the offset. Although all three parameters are vital to an effective coordination plan, the key parameter is the offset. The cycle length and phase splits are specific to each intersection. However, the offset is the basis of the relationships between each of the intersections in the system.

Offsets are programmed so that when traffic on the main street of the system reaches the upstream signal, it is turning green. In order for the offsets to be effective, the offsets have to be referenced from the same point in time. The reference point is a certain instance in the cycle. Ideally, all controllers in a system run the same cycle length. Therefore, if all of the controllers' cycle lengths begin at the same time, the offsets can be programmed effectively. Consequently, it is essential that all intersection clocks be synchronized to the nearest one second. This synchronization can be accomplished by either very accurate time close or physical interconnect. Physical interconnected closed loop systems are the preferred approach because it is easier to ensure synchronized times and it provides a mechanism for easily uploading and downloading timing plans.

\footnotetext{
${ }^{1}$ Gao, 1994.
} 
Once there are interconnected controllers at each intersection in the system, a master controller can be installed to send the clock synchronization signal. The master controller can also be used to call the coordination plans so that every intersection is running the same plan. Most traffic signal controller vendors have management software for their controllers. This software can be used to access each controller individually through a modem connection or direct connection. With a computer running the software connected to a master controller, only one connection is needed to access every controller in the system.

The premise of having a series of interconnected local controllers with a master controller that can be monitored remotely is known as a closed loop system. This manual covers every aspect of closed loop systems from designing the basic timing parameters to fine tuning the system after it is implemented in the field. Figure 1-1 shows the topics that will be covered in each chapter of this manual.

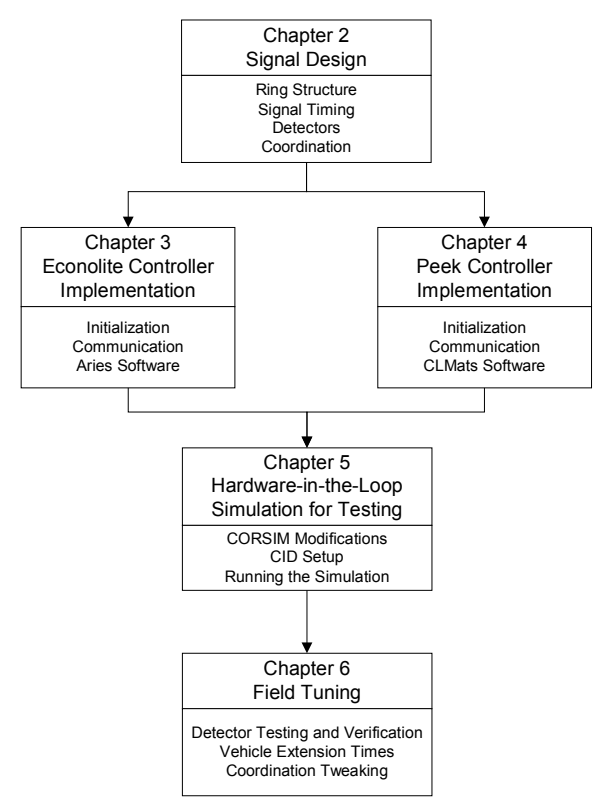

Figure 1-1 Chapter Concepts

Chapter 2 lays out the process for designing the different parameters of the traffic signal control for a system. Throughout the rest of the text, the "process for determining parameters for traffic signals and traffic signal systems" will be referred to as "design of a traffic signal system". First, the concept of the ring structure and laying out the phases for an intersection are discussed.

Then, guidance is provided for computing the basic timing parameters for each phase in the ring structure. The ring structure and the timing parameters provide enough information to configure a 
controller to run an intersection. However, the intersection and the system will run more efficiently after detection and coordination plans are added. Additional information for configuring detectors and a discussion on coordination are included in this chapter.

Chapter 3 and Chapter 4 outline the configuration of two types of controllers for a closed loop system. The two types of controllers are the Econolite controller and the Peek controller. Every step needed to set up a closed loop system is discussed. These steps include controller initialization, communication between the controllers, data entry, and remote monitoring software. The parameters calculated in Chapter 2 are incorporated into the data entry.

Chapter 5 provides information on testing the controllers once they are configured. All of the effort spent designing the system and configuring the controllers is virtually wasted if a crucial error is made during the data entry. This manual advocates the use of a hardware-in-the-loop simulation in a lab for testing the controllers in real-time. The simulation is possible through the use of a controller interface device (CID). Details about the simulation and the CID, along with how the hardware-in-the-loop simulation is conducted are included in this chapter. The key steps are modifying the CORSIM simulation, configuring the CIDs, and making the CID, computer, and controller connections.

Chapter 6 constitutes the final step in the implementation process and covers tuning the system after it is in the field. Procedures and checks are provided for the detectors, timing parameters, and coordination plans. 


\section{Chapter 2. Closed Loop System Design Procedures}

The purpose of this chapter is to provide guidance on designing controller timing parameters. The parameters are calculated based on intersection geometry ${ }^{2}$ and established standards in the traffic signal field ${ }^{3}$. Figure 2-1 illustrates the sequence of procedures for designing timing parameters (Chapter 2) and implementing them on Econolite (Chapter 3) and Peek (Chapter 4) controllers.

The first step in the signal timing design process is to select the ring structure based on the intersection geometry and the vehicle and pedestrian movements. Once the phases have been assigned in the ring structure, the timing intervals are calculated based on the geometry and standards. Although these timing intervals alone will allow an intersection to run, detection and coordination are added to increase the efficiency of the intersection and the system. The detection is used to react to approaching vehicles, as well as collect volume data. Coordination is implemented to provide progression through a series of signals. There are three main parameters that must be considered when programming coordination: split, cycle, and offset. Usually, the data needed for coordination is generated using an optimization package. The Indiana Department of Transportation commonly uses Synchro for these calculations. Upon completion of the design procedures, the system is implemented on a controller and tested in the field.

\footnotetext{
${ }^{2}$ MUTCD 2000.

${ }^{3}$ Kell, 1991.
} 


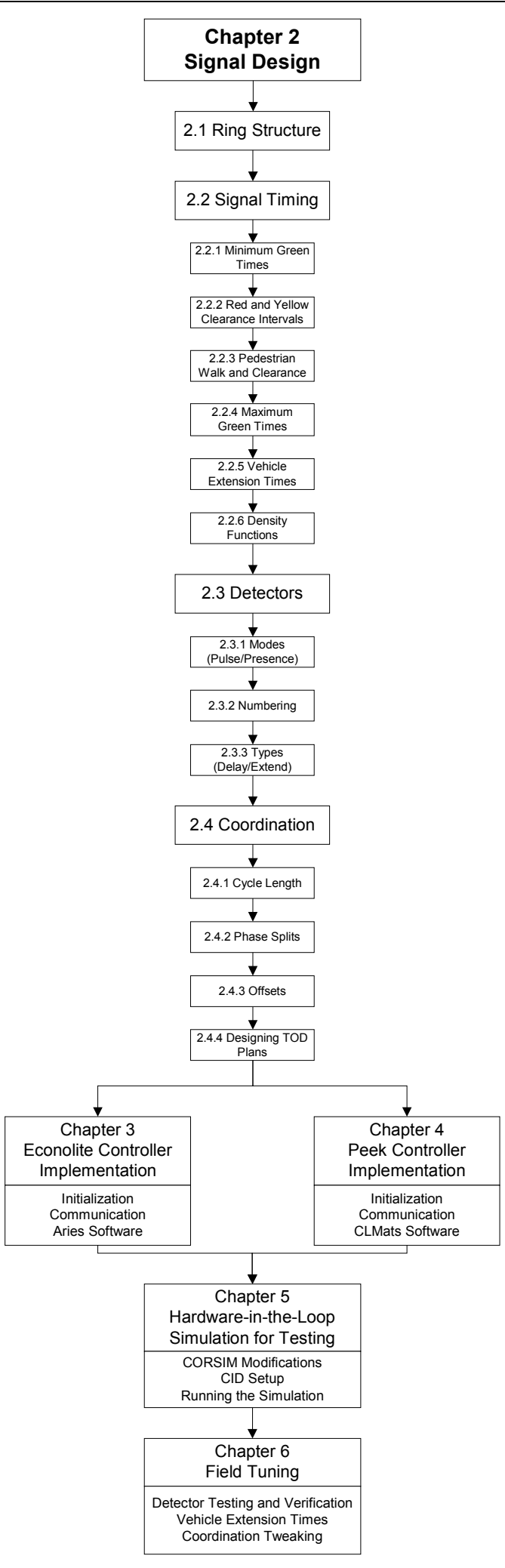

Figure 2-1 Chapter Concepts - Signal Timing Design Procedure 


\subsection{Ring Structures and Phases}

The fundamental diagram describing intersection signal concepts is the ring structure. Ring structures depict the order that intersection movements follow. Various ring structures can be used to provide lagging left-turns, split phasing, and multi-intersection control, but the standard ring structure consists of eight phases and is shown in Figure 2-2a. Phases 1-4 are in ring 1 and phases 5-8 are in ring 2. Two barriers are shown as thick vertical lines. Phases cannot run concurrently with phases on the other side of a barrier. Phases cannot run concurrently with phases in the same ring. By NEMA ${ }^{4}$ convention, even numbered phases are assigned to through movements and odd numbered phases are assigned to protected left-turn movements (Figure 2-2). Phase 2 is typically assigned to northbound or eastbound main street traffic. Subsequent even phase numbers increase clockwise. Protected left-turn phases are assigned with phase 1 adjacent to phase 6 . Subsequent odd phase numbers increase clockwise. Phase numbering can be checked with the seven-eleven rule. Approach movements should total 7 on the main street and 11 on the side street. For example, the northbound left-turn phase 5 plus the northbound through phase 2 equals 7 . This ring structure can be adapted to serve intersections that do not have four approaches, such as a T-intersection or an interchange ramp by omitting unused phases.

\footnotetext{
${ }^{4}$ NEMA TS2.
} 


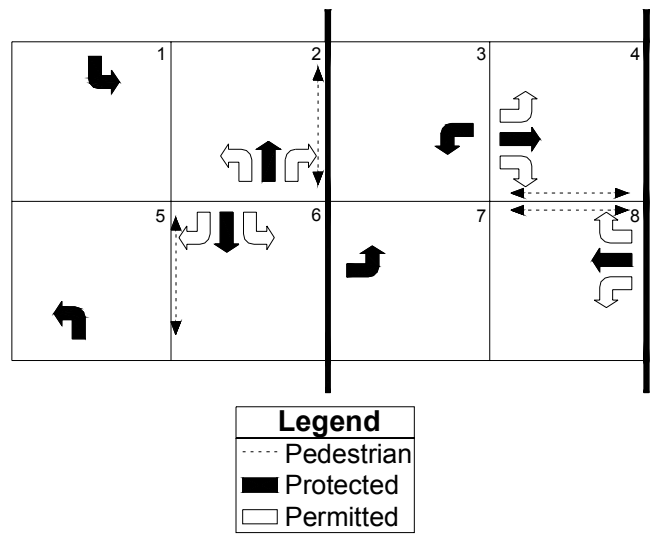

(a) 8 Phase Ring Structure

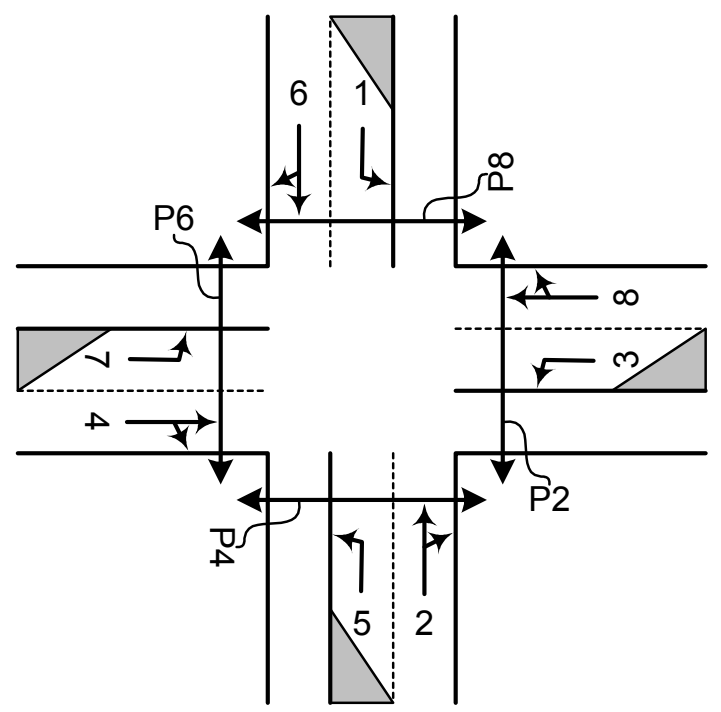

(b) Standard Intersection with Left-Turn Lanes

Figure 2-2 Standard Ring Configuration

Figure 2-3 depicts a minimum cycle length for the standard ring structure in Figure 2-2a.

Assuming the minimum green, yellow, and red times shown, the minimum cycle length would be 45 seconds. Ring 1 and Ring 2 individually total 45 seconds, but they are running concurrently.

\begin{tabular}{|c|c|c|c|c|c|c|c|c|c|c|}
\hline Min Green & Yellor & Red & Min Green & Yellow & Min Green & Yellow & Red & Min Green & Yellow & $R$ \\
\hline 5 & 3 & 1.5 & 10 & 4 & 5 & 3 & 1.5 & 7 & 4 & $=45 \mathrm{~s}$ \\
\hline 5 & 3 & 1.5 & 10 & 4 & 5 & 3 & 1.5 & 7 & 4 & $=45 \mathrm{~s}$ \\
\hline
\end{tabular}

Figure 2-3 Minimum Cycle Length

In Figure 2-2a, permitted left-turn movements are shown in the even-numbered phases as hollow arrows. This protected/permitted phasing sequence is implemented with a 5-section signal head (Figure 2-4). The left-turning traffic is first protected (Figure 2-4a) and then permitted (Figure $2-4 b)$. Some intersections may use protected only left-turns. In this case, the left-turn signal would be a 3-section head with a green arrow and circular yellow and red indications.

Some agencies use lead/lag left-turn operation. This allows the protected left-turn movement to be served after the through movement. If this is done, the order of the phases is merely switched in the ring structure, but not renumbered. Some situations such as diamond interchanges and 
split phase intersections require different phase order and barrier location. However, these cases are relatively infrequent.

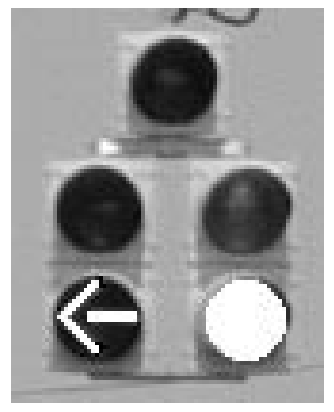

(a) Protected Left-turn Movement

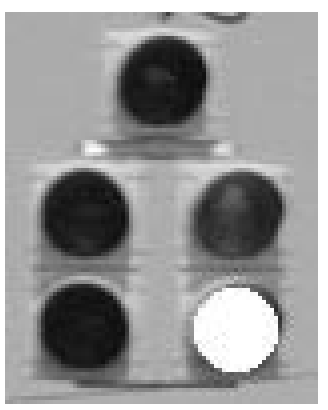

(b) Permitted Left-Turn Movement

Figure 2-4 Left-turn 5-section Signal Head

\subsubsection{Concurrent Pedestrian Phases}

For the ring structure shown in Figure 2-2a, pedestrian movements run concurrently with their adjacent through vehicle phase, so pedestrian phase 2 would run with vehicle phase 2 .

According to the MUTCD, traffic facing a circular green signal may proceed straight uninhibited or turn right or left, yielding the right-of-way to other lawful vehicles and pedestrians. Furthermore, in the absence of a pedestrian signal, pedestrians facing any green signal indication may proceed across the roadway, except when the signal indication is a turn arrow for a vehicular movement in conflict with the desired path of the pedestrian. ${ }^{5}$ However, there is frequent pedestrian confusion when they are facing the left-turn indication shown in Figure 2-4a when both the circular green and the conflicting arrow are shown concurrently. This sometimes results in pedestrian confusion with regards to who has the right-of-way. To eliminate any uncertainty, it is recommended that pedestrian signals always be used on all pedestrian movements where 5 -section heads would be visible to pedestrians.

\subsubsection{Exclusive Pedestrian Phases}

At intersections with very heavy right-turning traffic and heavy concurrent pedestrian movements, there is a significant opportunity for pedestrian/vehicle conflicts. Pedestrians can have exclusive phases if the pedestrian volumes are high enough, such as in a central business district. This type of pedestrian operation is often called a "Barnes Dance" after Harry Barnes, former traffic

\footnotetext{
${ }^{5}$ MUTCD 2000, Section 4D.04, Part A.
} 
commissioner for Denver and New York City who developed it in the 1950s. This exclusive pedestrian phase runs outside the ring structure and often causes difficulty in obtaining good system progression because the activation of the extra phase can cause an unusually long cycle length.

\subsubsection{Right-turn Phases}

If an intersection has exclusive right-turn lanes, the right-turn movements can be programmed to run concurrently with the adjacent left-turn movement. This type of operation is usually performed by an "overlap phase". Right-turn overlaps typically use a 5-section head as shown in Figure 2-5. When the overlap is on, the arrow is green, implying a protected movement. When the approach's through phase is green, there is no green arrow, implying a permitted movement.

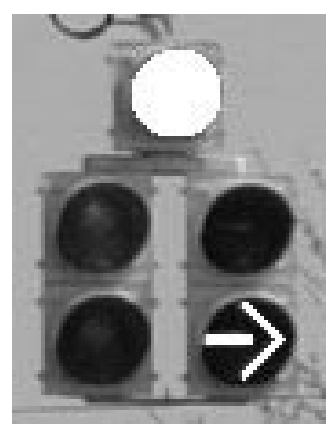

(a) Right-Turn Protected Overlap

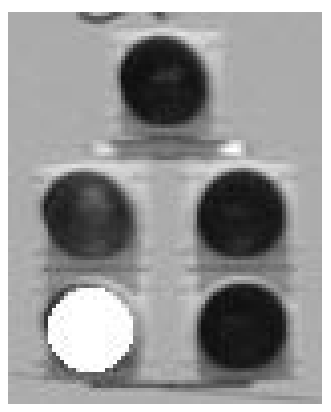

(b) Right-turn Permitted

Figure 2-5 Right-turn Overlap 5-Section Signal Head

The ring structure with right-turn overlaps looks like the one in Figure 2-6a. Permitted movements are shown with a hollow arrow, protected movements are shown with a solid or hatched arrow. Overlaps are assigned in the controller as A, B, C, and D. Overlap A would be adjacent to the through movement phase 2, as shown in Figure 2-6b. Right-turn overlap A would run with protected left-turn phase 3 . This movement would not cause any vehicular conflicts. Programming overlaps in the controller will be covered in Chapter 3 and Chapter 4. 

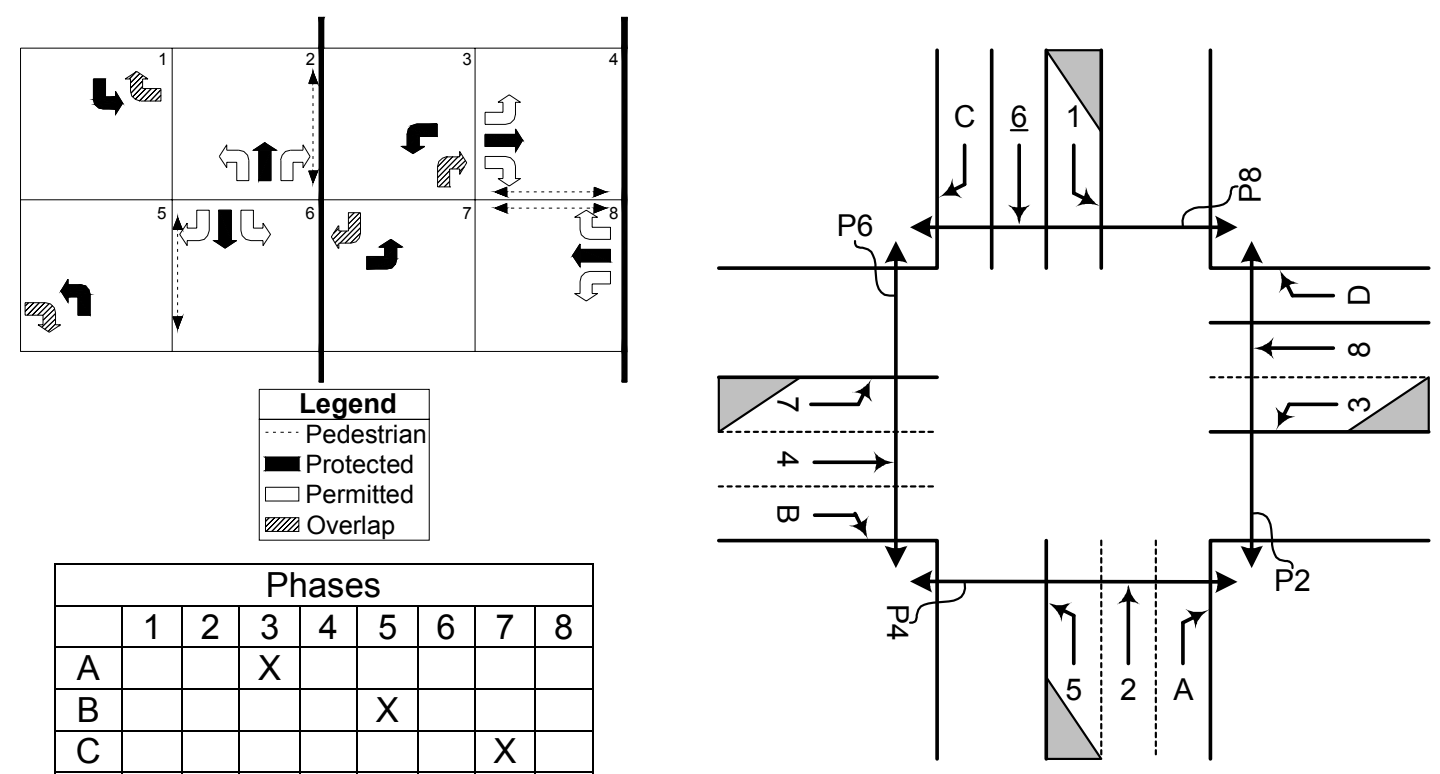

\begin{tabular}{|l|l|l|l|l|l|l|l|l|}
\hline \multicolumn{10}{|c|}{ Phases } \\
\hline & 1 & 2 & 3 & 4 & 5 & 6 & 7 & 8 \\
\hline A & & & $\mathrm{X}$ & & & & & \\
\hline B & & & & & $\mathrm{X}$ & & & \\
\hline C & & & & & & & $\mathrm{X}$ & \\
\hline D & $\mathrm{X}$ & & & & & & & \\
\hline
\end{tabular}

(b) Standard Intersection with Overlaps

Figure 2-6 Standard Ring Configuration with Overlaps 


\subsection{Timing Data}

Before the controller can be programmed, the timing data has to be calculated. Table 2-1 shows the important timing parameters for the first intersection in the system. Data for all five intersections can be found in Appendix B. The calculations in this Section are for intersection 1.

Table 2-1 Timing Parameters

\begin{tabular}{|c|c|c|c|c|c|c|c|c|c|}
\hline INT & DESCRIPTION (SEC) & $\mathbf{1}$ & $\mathbf{2}$ & $\mathbf{3}$ & $\mathbf{4}$ & $\mathbf{5}$ & $\mathbf{6}$ & $\mathbf{7}$ & $\mathbf{8}$ \\
\hline 1 & MINIMUM GREEN & 5 & 10 & 5 & 7 & 5 & 10 & 5 & 7 \\
\hline 1 & YELLOW CLEARANCE & 3.2 & 4.0 & 3.2 & 4.0 & 3.2 & 4.0 & 3.2 & 4.0 \\
\hline 1 & RED CLEARANCE & 2.0 & 1.5 & 2.0 & 1.5 & 2.0 & 1.5 & 2.0 & 1.5 \\
\hline 1 & PEDESTRIAN CLEARANCE & -- & 11 & -- & 11 & -- & 11 & -- & 11 \\
\hline 1 & PEDESTRIAN WALK & -- & 7 & -- & 7 & -- & 7 & -- & 7 \\
\hline 1 & MAX1 GREEN & 15 & 46 & 15 & 67 & 12 & 48 & 16 & 66 \\
\hline 1 & MAX2 GREEN & 15 & 46 & 15 & 67 & 12 & 48 & 16 & 66 \\
\hline 1 & MAX3 GREEN & 17 & 53 & 17 & 78 & 14 & 56 & 19 & 76 \\
\hline 1 & MAXIMUM EXTENSION & 1.0 & 3.5 & 1.0 & 5.5 & 1.0 & 4.0 & 1.5 & 5.0 \\
\hline 1 & VEHICLE EXTENSION & 1.4 & 5.0 & 1.4 & 5.0 & 1.4 & 5.0 & 1.4 & 5.0 \\
\hline 1 & TIME BEFORE REDUCTION & -- & 15 & -- & 22 & -- & 18 & -- & 25 \\
\hline 1 & TIME TO REDUCE & -- & 37 & -- & 54 & -- & 45 & -- & 61 \\
\hline 1 & MINIMUM GAP TIME & -- & 3.0 & -- & 3.0 & -- & 3.0 & -- & 3.0 \\
\hline 1 & SEC/ACTUATION & -- & -- & -- & -- & -- & -- & -- & -- \\
\hline 1 & MAXIMUM INITIAL & -- & -- & -- & -- & -- & -- & -- & -- \\
\hline
\end{tabular}

\subsubsection{Minimum green times}

The purpose of minimum green is to prevent a green indication from quickly turning on and off when that phase is served. Green times that are too short can lead to driver confusion and perhaps rear-end collisions. The minimum green is the guaranteed minimum time once a phase is started. However, a phase may stay on longer than the minimum. By convention, Indiana Department of Transportation uses the following minimum green times:

- All left-turn movements $=5$ seconds

- Main street through movements $=10$ seconds

- $\quad$ Side street through movements $=7$ seconds.

Some judgment should be used when applying these numbers. For example, if a side street through volume is very low, a minimum of 5 seconds might be used, or if a left-turn volume is very high, a minimum of 7 seconds may be used. 


\subsubsection{Clearance Intervals}

The purpose of the yellow clearance interval is to warn traffic of an impending change in the rightof-way assignment. The yellow clearance interval should be between 3-6 seconds, but may be longer. ${ }^{6}$ The yellow clearance and red clearance are calculated together as a total clearance. The total clearance permits vehicles that are within the intersection or so close to it that they cannot comfortably stop, to clear the intersection. It also permits those vehicles that can come to a comfortable stop to do so. An average speed of 40 miles per hour, unless otherwise posted, is assumed on the through movements. A speed of 30 miles per hour on the left-turn movements is also assumed, unless posted lower. ${ }^{7}$ The total clearance ${ }^{8}$ is calculated using Eq. 2-1.

$$
\text { Total Clearance }=T_{p}+\frac{1.47 * \mathrm{~V}}{2 \mathrm{a}}+\frac{\mathrm{W}+\mathrm{L}}{1.47 * \mathrm{~V}}
$$

where,

- $\mathrm{T}_{\mathrm{p}}$ is the perception time $=1$ second,

- $\mathrm{V}$ is vehicle speed $=$ average through vehicle speed on that approach in $\mathrm{mph}$,

- $a$ is the deceleration rate $=10 \mathrm{ft} / \mathrm{s}^{2}$,

- $\mathrm{W}$ is adjacent approach width = full width of cross street in feet,

- $L$ is the average vehicle length $=20$ feet.

\subsubsection{Yellow Clearance Interval}

The first two terms in Eq. 2-1 are used to calculate the yellow clearance time.

$$
\begin{aligned}
& \text { Yellow Clearance }=\mathrm{T}_{\mathrm{p}}+\frac{1.47 * \mathrm{~V}}{2 \mathrm{a}} \\
& \text { Yellow Clearance }=1+\frac{1.47 * 40}{20}=4.0 \mathrm{sec}
\end{aligned}
$$

\subsubsection{Red Clearance Interval}

The red clearance is the duration after every phase that all indications are red. This is extra time to make sure the intersection is clear before proceeding to the next phase. The red clearance should not be more than 6 seconds. ${ }^{9}$ The last term in Eq. 2-1 is used to calculate the red clearance.

\footnotetext{
${ }^{6}$ MUTCD 2000, Section 4D.10.

${ }^{7}$ There is some disagreement on this value. It may be as low as $15 \mathrm{mph}$ in some states.

${ }^{8}$ Kell, 1991.

${ }^{9}$ MUTCD 2000, Section 4D.10.
} 


$$
\begin{aligned}
& \text { Red Clearance }=\frac{\mathrm{W}+\mathrm{L}}{1.47 * \mathrm{~V}} \\
& \text { Red Clearance }=\frac{72+20}{1.47 * 40}=1.5 \mathrm{sec}
\end{aligned}
$$

\subsubsection{Pedestrian Times}

\subsubsection{Clearance Time}

The purpose of the pedestrian clearance time is to give a pedestrian that is just leaving the curb at the end of the walk signal enough time to reach at least the center of the farthest traveled lane or a median of sufficient width for the pedestrian to wait ${ }^{10}$. The pedestrian clearance interval is indicated by a flashing hand on the pedestrian signal shown in Figure 2-7a. This interval begins immediately at the end of the pedestrian walk interval shown in Figure 2-7b. The clearance interval may overlap with the yellow and red clearance times of the concurrent vehicle phase. A walking speed of 4 feet per second is typically used. ${ }^{11}$ The pedestrian clearance time is based on the width of the approach. Since all approaches at an intersection might not have the same width, all of the pedestrian clearance times may or may not be the same. Each approach to this intersection has the same number of lanes (see Figure B-3 in Appendix B). Assuming a lane width of 12 feet the pedestrian clearance time can be calculated as follows:

$$
\begin{aligned}
& \text { Actual Pedestrian Clearance Time }=\frac{(\# \text { of lanes } * \text { lane width })-.5 \text { lane width }}{\text { walking speed }} \\
& \text { Actual Pedestrian Clearance Time }=\frac{(6 * 12 \text { feet })-.5 * 12 \text { feet }}{4 \text { feet per second }}=16.5 \mathrm{~s}
\end{aligned}
$$

\footnotetext{
${ }^{10}$ MUTCD 2000, Section 4E.09.

${ }^{11}$ MUTCD 2000, Section 4E.09.
} 


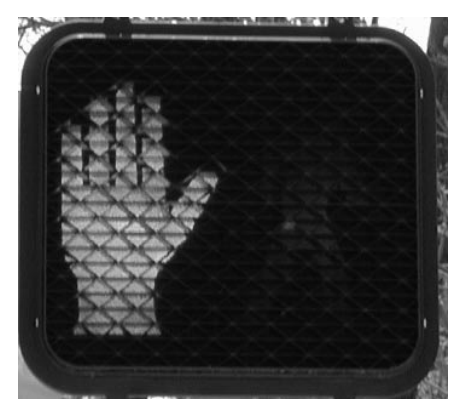

(a) Pedestrian Clearance (Flash)

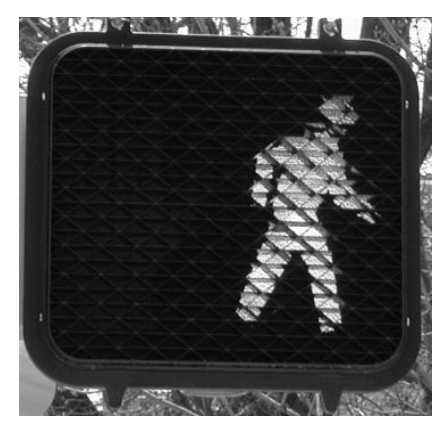

(b) Pedestrian Walk

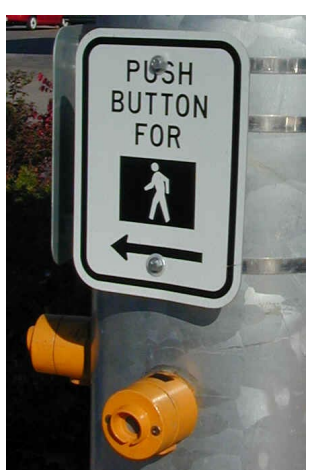

(c) Pedestrian Detector

Figure 2-7 Pedestrian Signal

\subsubsection{Walk Time}

The purpose of pedestrian walk time is to give the pedestrian adequate time to leave the curb or shoulder before the pedestrian clearance time begins. According to the MUTCD, the pedestrian walk interval should be:

- $\quad$ Most intersections $=7$ seconds

- $\quad$ Special cases $=4$ second minimum $^{12}$

- However, for pedestrian safety, the highest possible walk time is sometimes used. To determine the maximum walk time for each pedestrian interval, examine the coordination splits for the corresponding phases. Use the shortest split for each phase 2, 4, 6, and 8 and subtract the pedestrian clearance time for that phase that is calculated next. If there is more than 7 seconds of green time left for the walk interval, this maximum could be used. However, if walk times and pedestrian clearance times are set too high, they can force an intersection out of coordination because the full times have to be served when a pedestrian call is received. High pedestrian times can also prevent the side street (uncoordinated) phases from gapping out early because the phase is extended the duration of pedestrian time, regardless of vehicle calls. The procedure for calculating maximum walk times that will not violate coordination constraints are shown below for phase 2.

$\mathrm{Ph} 2 \mathrm{Max}$ Walk Time = shortest split - controller pedestrian clearance- yellow - red

Ph 2 Max Walk Time $=(32 \% * 90 \mathrm{~s})-12 \mathrm{~s}-4 \mathrm{~s}-0.5 \mathrm{~s}=12$ seconds

${ }^{12}$ MUTCD 2000 Section 4E.09 
Since the phase split time includes the yellow and red clearance times, these intervals must be subtracted, in addition to the controller pedestrian clearance time, to get the maximum walk time. Similar calculations can be made for the other pedestrian phases 4,6 , and 8 .

\subsubsection{Maximum Green Times}

\subsubsection{Max1 and Max2}

Two different maximum green times can be entered as max1 and max2. A Time-of-Day program allows selection of $\max 1$ and $\max 2$ times for different times of the day. For example, max 1 times could be used during the morning peak period, while max 2 is used in the evening peak period. Another situation might be to use a higher set of max 2 times while the system is in coordination and lower max 1 times used while the system is running free, say at night. Note that when the intersection runs free, the max timer does not start counting down until an opposing call is received.

If a coordination error occurs, sometimes the intersection is forced to run free, where calls are served on a first-come, first-served basis. If the maximum green times were set too high, one busy approach could keep the green indication for several minutes. Conversely, if the max times were set very low a busy intersection could become very congested due to the very short cycles that would increase the "lost time". "Lost time" refers to the theoretically unused time in a cycle that is yellow and red clearance time and start-up lost time (time to accelerate).

As will be shown in Chapter 3 and Chapter 4 in the coordination menu, the maximum green times can be inhibited. If the maximum green times are lower than the splits and they are not inhibited, the green phases will terminate due to max out and the intersection could fall out of coordination. It may be desirable to set the maximum green times close to the phase splits. If this is done and coordination is lost, the intersection will perform similarly to when it was in coordination. The following method is recommended to calculate $\max 1$ and $\max 2$ green times:

- $\operatorname{Max} 1=1.3 *$ morning peak split time

- $\operatorname{Max} 2=1.3^{*}$ evening peak split time

- Where the split time is obtained from a design package such as the Highway Capacity Software, Signal 2000, or Synchro.

In the absence of adequate data to calculate the splits, the following guides can be used:

- Main street max green $=50$ seconds

- Side street max green $=40$ seconds

- All left-turn max greens $=35$ seconds . 


\subsubsection{2. $\quad \operatorname{Max} 3$}

Max3 green time is used during free operation to extend the green interval for a busy intersection. If a green phase terminates due to a max out in two successive cycles, the maximum green will extend a set amount of time until the max 3 time is reached. Then, if the phase terminates due to a gap out for two successive cycles, the maximum green will either reduce the set amount of time until it reaches max 1 or max 2 or the max timer will be reset. This is dependent on the controller being used. The max3 parameters are calculated as follows:

- $\operatorname{Max} 3=1.5^{*}$ split time

- Maximum extension = $1 / 2(\operatorname{Max} 3-\operatorname{Max} 1)$.

If there is not enough data to calculate the split times, the guides can be used to calculate the max3 parameters:

- $\quad$ Main street $\max 3=75$ seconds

- Main street max ext $=12$ seconds

- Side street $\max 3=60$ seconds

- $\quad$ Side street $\max$ ext $=10$ seconds

- All left-turn $\max 3=52$ seconds

- $\quad$ All left-turn max ext = 8 seconds.

\subsubsection{Extension Times}

\subsubsection{Vehicle Extension for Stopbar Detection}

The purpose of the vehicle extension time is to extend the green indication a certain amount of time after a call has been received during the green phase and after the minimum green time has been served. This parameter usually accounts for gaps between vehicles at actuated approaches. The green interval is extended once the vehicle leaves the detection zone. If the extension is set to 3 seconds, the phase is extended for 3 seconds whenever a call is received. Figure 2-8 illustrates the cycle performance when the green is extended by vehicle calls. When the gap between vehicles is greater than the vehicle extension time, the actuated phase terminates. 


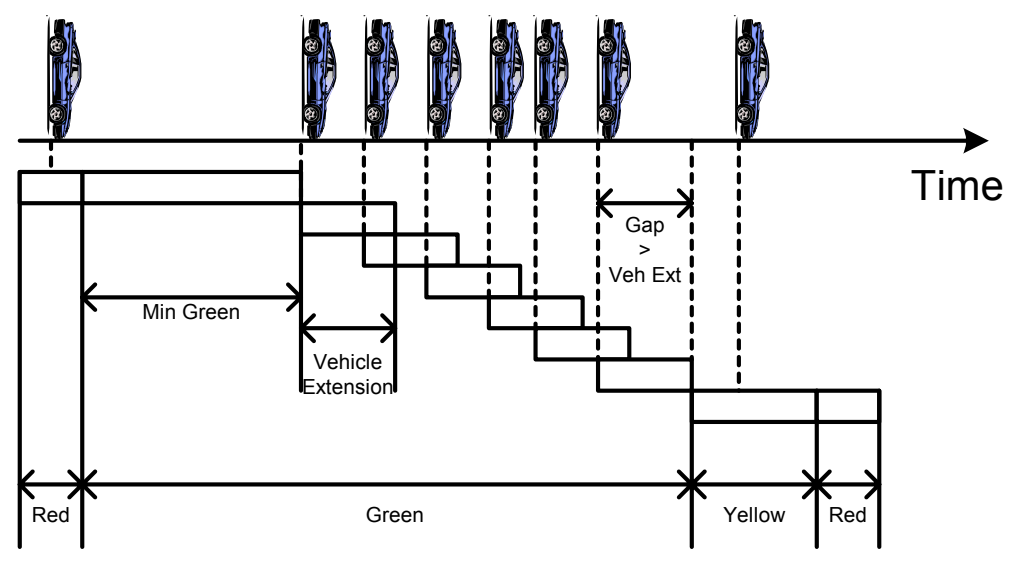

Figure 2-8 Vehicle Extension

It is recommended that the controller gap time be set so that 3 seconds of allowed gap is provided. This minimizes the risk of premature phase termination caused by slow or heavy vehicles. Since the extension time does not start timing until the vehicle call is lost, there is essentially already a vehicle gap allowance when presence detection is used while the vehicles travels over the length of the loop detector. This gap allowance can be calculated based on the speed of the vehicles and the length of the detection zone. For example, if the average speed of vehicles on the approach is $40 \mathrm{mph}$, a vehicle is 20 feet long and the detector is 20 feet long, the gap allowance can be calculated as follows:

$$
\begin{aligned}
& \text { Gap Allowance }=\frac{\text { Vehicle Length }+ \text { Detector Length }(\text { feet })}{1.47 * \text { Average Vehicle Speed }} \\
& \text { Gap Allowance }=\frac{20+20}{1.47 * 40}=0.7 \text { seconds }
\end{aligned}
$$

The gap allowance is summarized in Table 2-2. This table shows gap allowances for various vehicle speeds and detection lengths. The vehicle extension that is entered into the controller if the desired gap is 3 seconds should then be calculated with Eq. 2-7.

Controller Vehicle Extension = Total Desired Gap - Gap Allowance

Controller Vehicle Extension $=3.0-0.7=2.3$ seconds 
Table 2-2 Allowed Gap By Presence

\begin{tabular}{|c|c|c|c|c|c|c|c|c|c|}
\hline $\begin{array}{c}\text { Approach } \\
\begin{array}{c}\text { Speed } \\
\text { (mph) }\end{array}\end{array}$ & $\mathbf{2 0}$ & $\mathbf{3 0}$ & $\mathbf{4 0}$ & $\mathbf{5 0}$ & $\mathbf{6 0}$ & $\mathbf{7 0}$ & $\mathbf{8 0}$ & $\mathbf{9 0}$ & $\mathbf{1 0 0}$ \\
\cline { 2 - 11 } & 0.7 & 1.0 & 1.4 & 1.7 & 2.0 & 2.4 & 2.7 & 3.1 & 3.4 \\
\hline $\mathbf{2 0}$ & 0.5 & 0.8 & 1.1 & 1.4 & 1.6 & 1.9 & 2.2 & 2.4 & 2.7 \\
\hline $\mathbf{2 5}$ & 0.5 & 0.7 & 0.9 & 1.1 & 1.4 & 1.6 & 1.8 & 2.0 & 2.3 \\
\hline $\mathbf{3 0}$ & 0.5 & 0.6 & 0.8 & 1.0 & 1.2 & 1.4 & 1.6 & 1.7 & 1.9 \\
\hline $\mathbf{3 5}$ & 0.4 & 0.6 & 0.8 & .9 & \\
\hline $\mathbf{4 0}$ & 0.3 & 0.5 & 0.7 & 0.9 & 1.0 & 1.2 & 1.4 & 1.5 & 1.7 \\
\hline $\mathbf{4 5}$ & 0.3 & 0.5 & 0.6 & 0.8 & 0.9 & 1.1 & 1.2 & 1.4 & 1.5 \\
\hline $\mathbf{5 0}$ & 0.3 & 0.4 & 0.5 & 0.7 & 0.8 & 1.0 & 1.1 & 1.2 & 1.4 \\
\hline $\mathbf{5 5}$ & 0.2 & 0.4 & 0.5 & 0.6 & 0.7 & 0.9 & 1.0 & 1.1 & 1.2 \\
\hline
\end{tabular}

In Figure 2-9, the actual gap between the vehicles is 120 feet, which is 2 seconds at 40 miles per hour. However, even though the gap is 2 seconds, the trailing vehicle only needs 1.3 seconds to reach the detector and activate it. The extra 0.7 seconds of gap allowance is due to the 20 foot detector and the 20 foot vehicle.

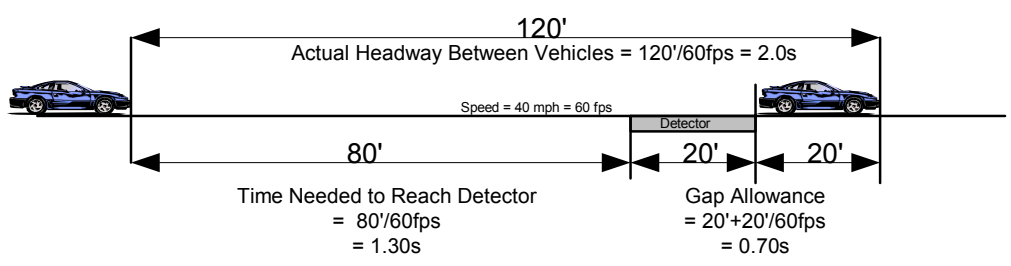

Figure 2-9 Gap Relationships

\subsubsection{Vehicle Extension for Advance Detection}

Vehicle extension is configured differently if only advance detection is used at an intersection. In this case, the extension time should allow the vehicle enough time to reach the intersection. If a car crosses a detector 200 feet from the intersection traveling 45 miles per hour, the vehicle extension time would be approximately 3.0 seconds, as shown in Eq. 2-8.

$$
\begin{aligned}
& \text { Vehicle Extension }=\frac{\text { Detector Distance from Stopbar }(\text { feet })}{1.47 * \text { Speed Limit }(\mathrm{mph})} \\
& \text { Vehicle Extension }=\frac{200 \text { feet }}{1.47 * 45 \mathrm{mph}}=3.0 \text { seconds }
\end{aligned}
$$

Table 2-3 gives the time needed to get from the advance detector to the stopbar for given speeds. For example, to obtain the extension for the previous example, find $45 \mathrm{mph}$ in the first column of the table. In the $45 \mathrm{mph}$ row, the number closest to 200 is 198 . The extension time for 198 feet is shown at the top of the column as 3 seconds. 
Table 2-3 Maximum Detector Setback (ft) for Various Speeds and Gap Extension Times

\begin{tabular}{|c|c|c|c|c|c|c|c|}
\hline \multirow{2}{*}{ Speed } & \multicolumn{7}{|c|}{ Extension Time } \\
\hline & $1 \mathrm{sec}$ & $2 \mathrm{sec}$ & $3 \mathrm{sec}$ & $4 \mathrm{sec}$ & $5 \mathrm{sec}$ & $6 \mathrm{sec}$ & $7 \mathrm{sec}$ \\
\hline $20 \mathrm{mph}$ & 29 & 59 & 88 & 117 & 147 & 176 & 205 \\
\hline $25 \mathrm{mph}$ & 37 & 73 & 110 & 147 & 183 & 220 & 257 \\
\hline $30 \mathrm{mph}$ & 44 & 88 & 132 & 176 & 220 & 264 & 308 \\
\hline $35 \mathrm{mph}$ & 51 & 103 & 154 & 205 & 257 & 308 & 359 \\
\hline $40 \mathrm{mph}$ & 59 & 117 & 176 & 235 & 293 & 352 & 411 \\
\hline $45 \mathrm{mph}$ & 66 & 132 & 198 & 264 & 330 & 396 & 462 \\
\hline $50 \mathrm{mph}$ & 73 & 147 & 220 & 293 & 367 & 440 & 513 \\
\hline $55 \mathrm{mph}$ & 81 & 161 & 242 & 323 & 403 & 484 & 565 \\
\hline $60 \mathrm{mph}$ & 88 & 176 & 264 & 352 & 440 & 528 & 616 \\
\hline $65 \mathrm{mph}$ & 95 & 191 & 286 & 381 & 477 & 572 & 667 \\
\hline
\end{tabular}

\subsubsection{Density Functions}

These parameters are used with gap reduction settings. The purpose is to vary the amount of time between vehicle calls before a phase will gap out. Typically, these settings are only programmed for phases that have speeds greater than 40 miles per hour. The beginning point, or maximum gap, is the vehicle extension. If the vehicle extension that was calculated in Section 2.2.5 or 2.2.5.2 is less than the recommended maximum gap, the vehicle extension should be increased to the maximum gap. The gap reduction should be configured to begin once $33 \%$ of the max time for that phase has elapsed. The reduction continues until the minimum gap is reached at $80 \%$ of the max time. Max 1 or Max 2 times should be used to calculate the parameters since Max3 might not be in effect. The recommended guidelines for gap control include the following:

- Programmed for phases that have speeds $>40$ miles per hour

- Maximum Gap $=$ Vehicle Extension $=5$ seconds

- Minimum Gap = 3 seconds

- $\quad$ Time Before Reduction $=33 \%$ of max time

- $\quad$ Time To Reduce $=80 \%$ of max time.

When advance detection is the only detection used at an intersection, the seconds/actuation parameter is very important. If cars queue up to the detector during the red phase and no additional vehicles arrive to extend the green, there is a possibility of the minimum time being served without completely discharging the queue. By using the seconds/actuation parameter, the minimum green time is extended a certain number of seconds per each actuation. The numbers used for seconds/actuation and maximum initial time are based on the distance of the detection from the intersection and the number of lanes:

- $\quad$ Maximum Initial = see Table 2-4 
- Seconds/Actuation = maximum initial divided by total number of cars stored.

An example calculation is shown below assuming a 3-lane approach with 220 feet between the stopbar and the advance detectors. In general, the average car length can be assumed as 20 feet, unless there is a high volume of trucks.

Maximum Initial $=26.8$ seconds $($ from Table $2-4)$

Seconds $/$ Actuation $=\frac{26.8 \text { seconds }}{3 \text { lanes } * 11 \text { cars per lane }}=0.8$

Similarly, if there were only two through lanes, the maximum initial would be the same, but the seconds/actuation would be:

Seconds $/$ Actuation $=\frac{26.8 \text { seconds }}{2 \text { lanes } * 11 \text { cars per lane }}=1.2$

Table 2-4 Variable Initial Green Parameters

\begin{tabular}{|c|c|c|c|}
\hline $\begin{array}{c}\text { Storage Between } \\
\text { Stopbar and Back Loop } \\
\text { (1 lane) }\end{array}$ & $\begin{array}{c}\text { Distance to Back } \\
\text { Loop } \\
\text { (20/vehicle) }\end{array}$ & $\begin{array}{c}\text { Discharge } \\
\text { Headway } \mathbf{1 3}^{\mathbf{3}} \\
\text { (sec) }\end{array}$ & $\begin{array}{c}\text { Maximum } \\
\text { Initial (sec) }\end{array}$ \\
\hline 1 & 20 & 3.8 & 3.8 \\
\hline 2 & 40 & 3.1 & 6.9 \\
\hline 3 & 60 & 2.7 & 9.6 \\
\hline 4 & 80 & 2.4 & 12.0 \\
\hline 5 & 100 & 2.2 & 14.2 \\
\hline 6 & 120 & 2.1 & 16.3 \\
\hline 7 & 140 & 2.1 & 18.4 \\
\hline 8 & 160 & 2.1 & 20.5 \\
\hline 9 & 180 & 2.1 & 22.6 \\
\hline 10 & 200 & 2.1 & 24.7 \\
\hline 11 & 220 & 2.1 & 26.8 \\
\hline 12 & 240 & 2.1 & 28.9 \\
\hline 13 & 260 & 2.1 & 31.0 \\
\hline 14 & 280 & 2.1 & 33.1 \\
\hline 15 & 300 & 2.1 & 35.2 \\
\hline 16 & 320 & 2.1 & 37.3 \\
\hline 17 & 340 & 2.1 & 39.4 \\
\hline 18 & 360 & 2.1 & 41.5 \\
\hline 19 & 380 & 2.1 & 43.6 \\
\hline 20 & 400 & 2.1 & 45.7 \\
\hline
\end{tabular}

${ }^{13}$ Greenshields 


\subsection{Detectors}

Coordinated systems do not require detection to operate. However, with increased detection comes increased efficiency. Detection can be used at an intersection to both detect vehicles and collect volume, speed, and occupancy data. In most circumstances, the detectors are already installed and the controller programmer just needs to assign the detectors to their correct phases and configure them correctly. The current INDOT standard drawings use the labeling scheme shown in Figure 2-10. The proposed tagging scheme is shown in Figure 2-11. This scheme coincides with the NEMA phase numbering scheme. These labels are only used for tagging the detector wires for reference in the cabinet. These labels need to be converted into channel numbers for use in the controller. This process is much easier if the detectors are numbered using a standard scheme that can be remembered. Different agencies may have different methods of numbering detectors.

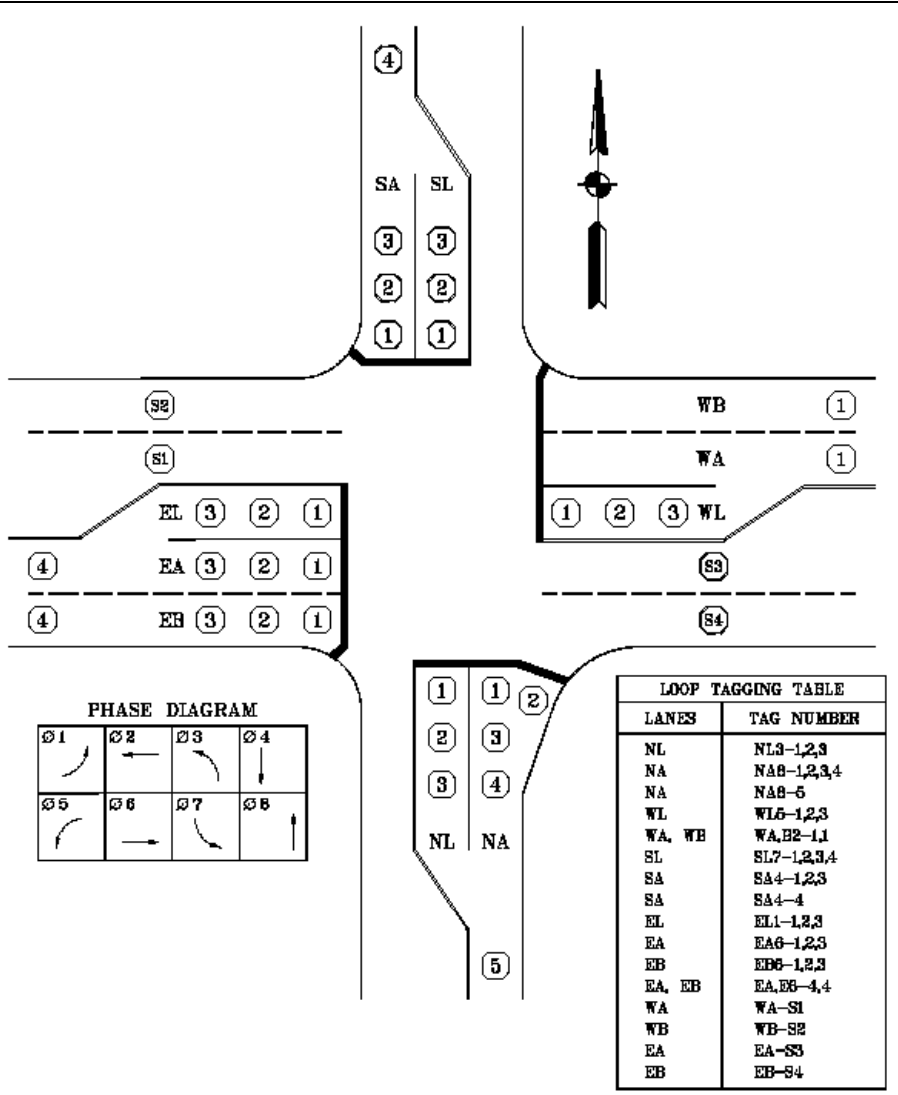

Figure 2-10 INDOT Loop Tagging Convention 


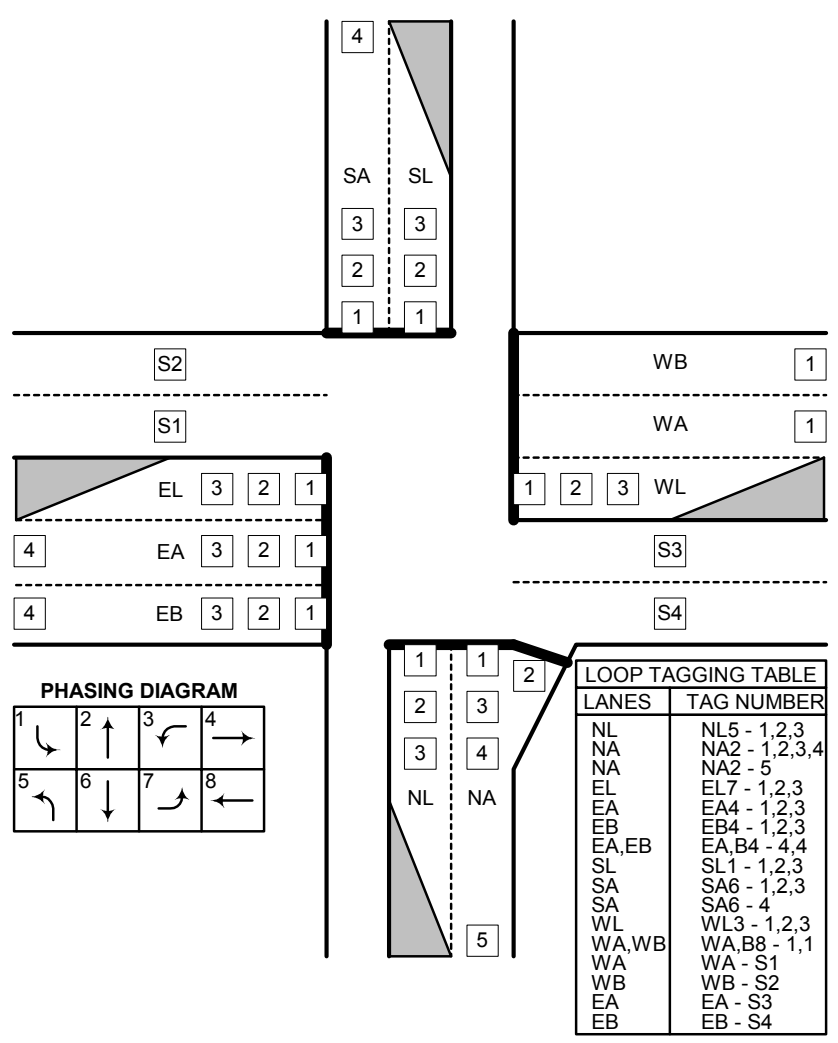

Figure 2-11 Proposed Loop Tagging Convention

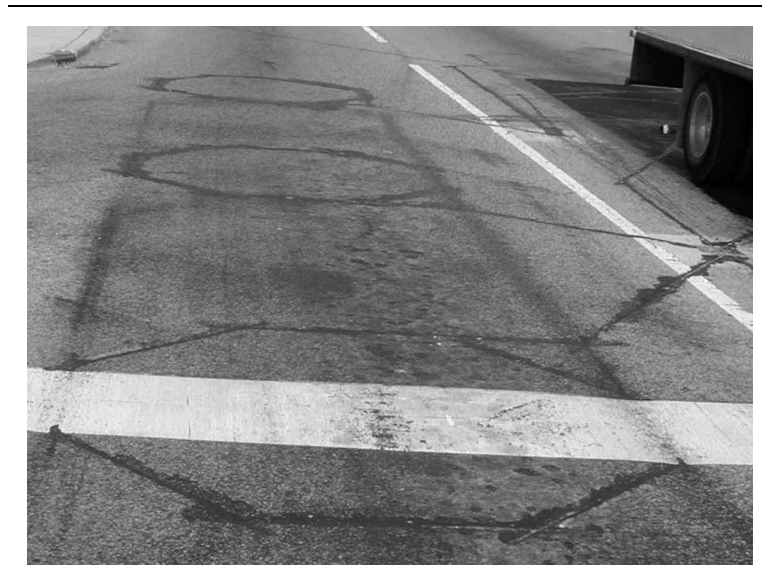

(a) 6' X 6' Loop Detectors

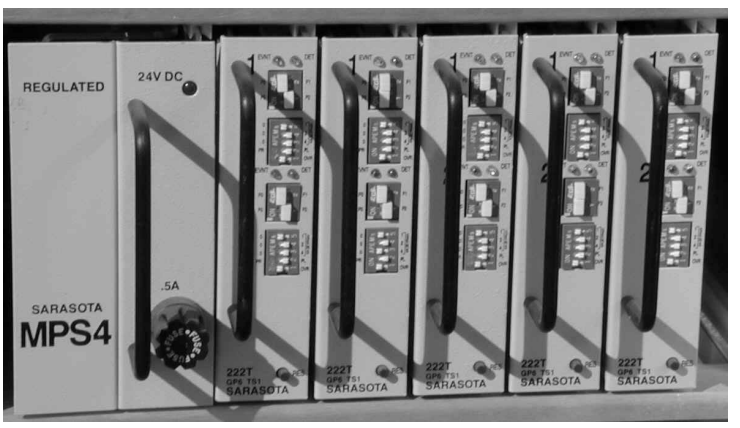

(b) Loop Amplifier Cards

Figure 2-12 Loop Detector Equipment

Figure 2-12a shows standard 6' $\times 6^{\prime}$ loop detectors that are installed in the pavement. This type of detection responds to a change of inductance in its proximity. The loops are connected in the signal cabinet to amplifier cards (Figure 2-12b). The amplifier cards monitor the change in 
inductance and decide whether or not a car is present. If there is a car present, the amplifier card sends a signal to the controller and the controller receives it as a call. Most amplifier cards can be configured for both sensitivity and pulse/presence. The sensitivity setting controls how far away from the edges of the loop a vehicle triggers the detector. The pulse/presence setting has different applications described in the next section.

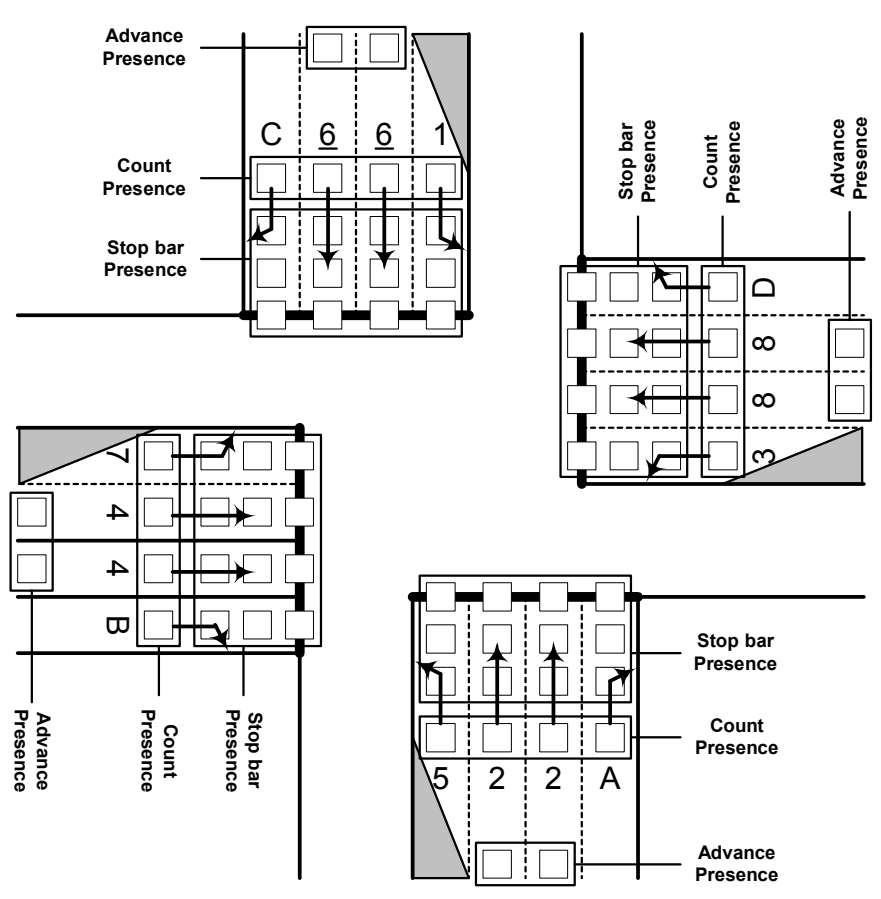

Figure 2-13 Four-legged Intersection with Four-Lane Approaches

\subsubsection{Detector Modes}

\subsubsection{Presence detection}

Presence detection is the most common setting used on amplifier cards, as well as the only setting used by INDOT at signalized intersections. When a presence amplifier card places a call, the call remains until the vehicle is no longer detected. Presence detection shall be used on all stopbar, count, and advance detectors, as shown in Figure 2-13. Figure 2-14 illustrates how a detector operates for both presence and pulse mode. As seen in Figure 2-14, presence detection stays on as long as there is a car over the loop. 


\subsubsection{Pulse detection}

If an amplifier card is set to pulse detection, a call is placed that lasts a set amount of time, approximately $110 \mathrm{~ms}$. Therefore, if a car pulls over a loop detector and stops, one call will be placed for a fraction of a second, as opposed to the call staying on in presence mode. As seen in Figure 2-14, the pulse detector is only on for a set amount of time, regardless of the car still being above the detector. However, when another car enters the detection zone, the call is placed again. Pulse setting is sometimes used for count detectors and raceway detectors.

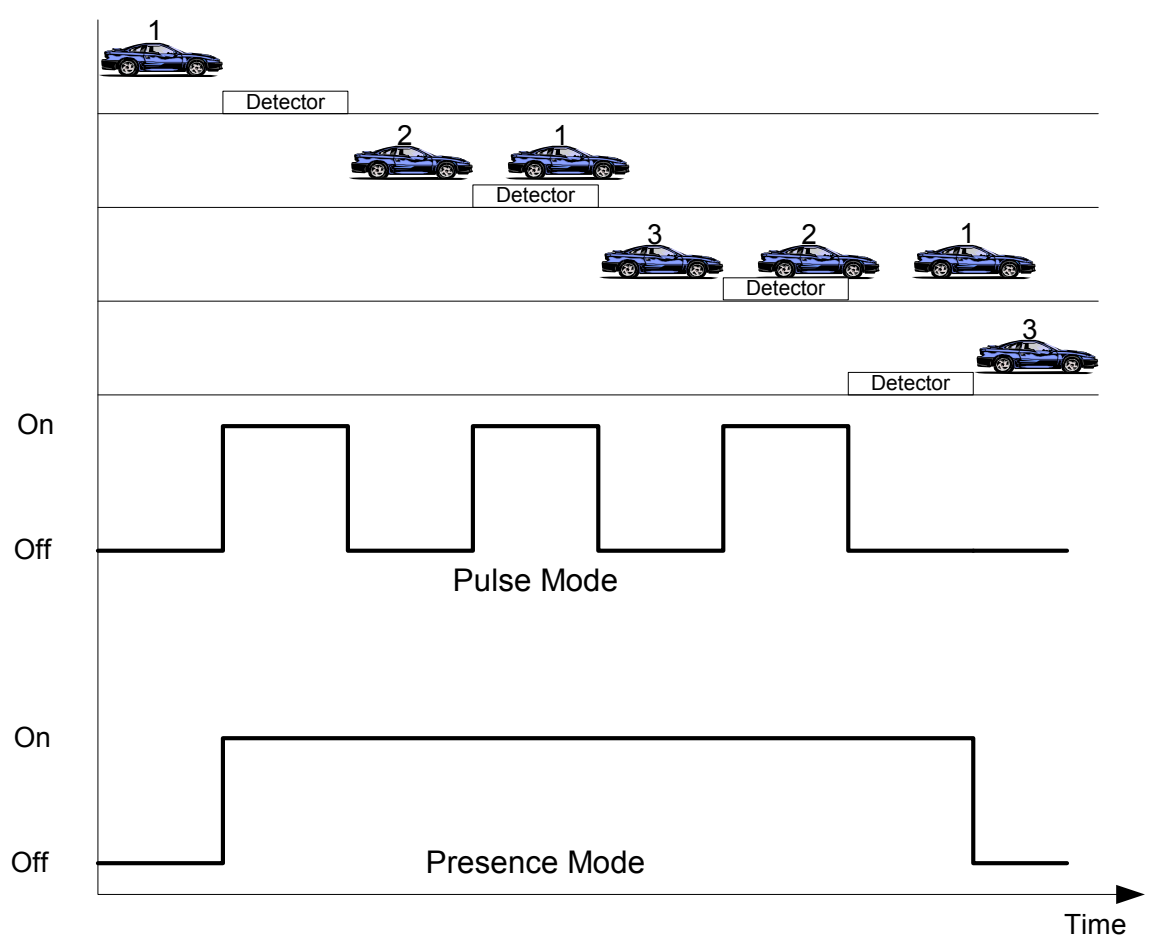

Figure 2-14 Pulse versus Presence Detection

\subsubsection{Detector numbering}

\subsubsection{TS2 Type 1 Conditions}

Figure 2-15 shows the detector numbering scheme for a standard approach. Typically, the approach will have either stopbar detection or advance detection, but rarely both. Therefore, they are shown with the same detector numbers. The two through lanes and the right-turn lane stopbar detectors are all assigned to phase 2. However, they are assigned to different channels. Starting with the inner lane, this detector is channel 2, corresponding to phase 2 . The count detector channel in that lane is obtained by adding 8 . The stopbar detector in the adjacent lane is 
obtained by adding 8 again. The remaining detector channels are obtained by adding 8 again. The left-turn lane presence detection is wired together to channel 5 , corresponding with phase 5 . The count detector is channel 13 . Only TS2 Type 1 controllers can have non-proprietary detectors numbered higher than 20.

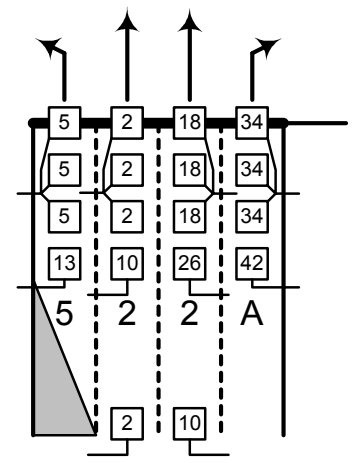

Figure 2-15 Detector Configuration

\subsubsection{TS2 Type 2 Conditions}

When running a hardware-in-the-loop simulation a different detector numbering scheme may be required. The maximum number of detectors that can be connected to a TS2 Type 2 controller via the 24 volt connector is 20 . Therefore, the detector numbers can only go up to 20 . In Figure $2-15$, the detectors that are assigned numbers over 20 will have to be modified. There is no real convention for the renumbering, but consistency is recommended.

\subsubsection{Detector Types}

In most controllers, detectors can be configured as different types. One variation that is commonly used is placing a delay on a detector. A situation that this might be used is a detector in an exclusive right-turn lane. If a detector has a delay of 10 seconds, a vehicle call during the red interval will not be recognized until 10 seconds has passed and the call is still present. If the vehicle leaves, or turns right on red, the call will not be placed. The delay is disabled during the green interval. This feature is only used to improve the intersection performance. Other types of detectors include stopbar detectors that only place calls while their corresponding phases are red, and extend detectors that will leave a call on for a set amount of time after the vehicle has left the detection zone. 


\subsection{Coordination}

Coordination plans can be designed using various software packages, such as Synchro or Passer. Intersection geometry, volumes, and signal timings are input into the software to obtain optimal cycle lengths, split times, and offsets. However, configuration of coordination is much more complicated than just entering these three parameters obtained from an optimization program. The implementation of these parameters will be covered in the next two chapters.

If various vehicle counts are available for the system, different coordination plans can be used at different times of the day. The different coordination plan can be turned on using a time-of-day program in the controller. INDOT uses four different coordination plans on Monday through Friday and one coordination plan on Saturday and Sunday. During the late night and early morning hours, most signals run in free mode with no coordination. Table 2-5 shows the schedule of different plans that may be used. Of course, the time periods shown in Table 2-5 are recommended in the absence of detailed data describing the start and end of various traffic patterns. Each of these plans will have a cycle, offset, and split designed using an optimization program.

Table 2-5 Coordination Plans by Time-of-Day

\begin{tabular}{|c|c|c|c|c|c|c|c|}
\hline Time & Sun & Mon & Tues & Wed & Thu & Fri & Sat \\
\hline 6am-9am & \multirow{4}{*}{ Plan 5} & Plan 1 & Plan 1 & Plan 1 & Plan 1 & Plan 1 & \multirow{4}{*}{ Plan 5} \\
\hline 9am-3pm & & Plan 2 & Plan 2 & Plan 2 & Plan 2 & Plan 2 & \\
\hline $3 p m-6 p m$ & & Plan 3 & Plan 3 & Plan 3 & Plan 3 & Plan 3 & \\
\hline 6pm-10pm & & Plan 4 & Plan 4 & Plan 4 & Plan 4 & Plan 4 & \\
\hline 10pm-6am & Free & Free & Free & Free & Free & Free & Free \\
\hline
\end{tabular}

\subsubsection{Cycle Length}

A cycle is a transition through the phases in a ring structure in a coordination plan. The cycle length is the time measured from the end of the coordinated phase (typically the main through movement) to the end of the coordinated phase the next time it is served. The cycle length is a set parameter in coordination plans. This duration is typically in the range of 60 to 180 seconds. During periods of light traffic demand, shorter cycle lengths are used to reduce delay. During periods of heavier traffic demand, longer cycle lengths are used. In order to achieve progression through a system, the cycle lengths must be the same. The only exception to this would be if an intersection were half-cycled - running a cycle length that is exactly half of what the rest of the system is running. 


\subsubsection{Phase Splits}

The phase splits are the time (or \% of time) devoted to each phase of the cycle. The splits include green, yellow, and all red times. For a standard ring structure, the sum of the splits in Ring 1 must be $100 \%$. Similarly the sum of the splits in Ring 2 must be $100 \%$. In addition, for a standard ring structure,

- $1+2=5+6$

- $3+4=7+8$.

\subsubsection{Force Off Times}

Internally, controllers use a concept of force off points for the coordinated phases. The force off times are computed from the splits and serve as "not to exceed" points in the cycle by which time the phase must have "forced off" in order to be able to reach the coordinated phase on schedule. Historically, force off times were manually calculated and entered into the controller. In more recent controllers, users directly enter the splits into the controller and the force off times are automatically calculated. Figure 2-16a shows a ring where all the phases extend to their force off points due to high traffic volumes. This results in the coordinated phases (2\&6) running for their minimum split time. Figure $2-16 \mathrm{~b}$ shows a ring where there is lighter traffic demand and the noncoordinated phases do not extend to the force off points. Notice the start (Point $\mathrm{S}$ ) of the coordinated phase is much earlier in Figure 2-16b than in Figure 2-16a.

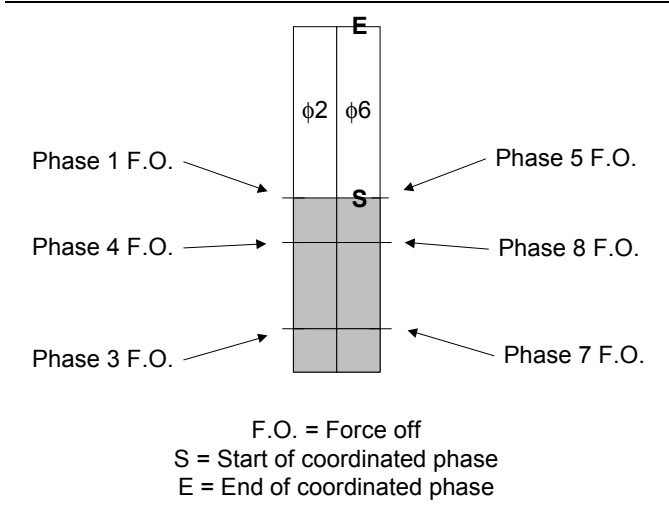

(a) Phases Max Out

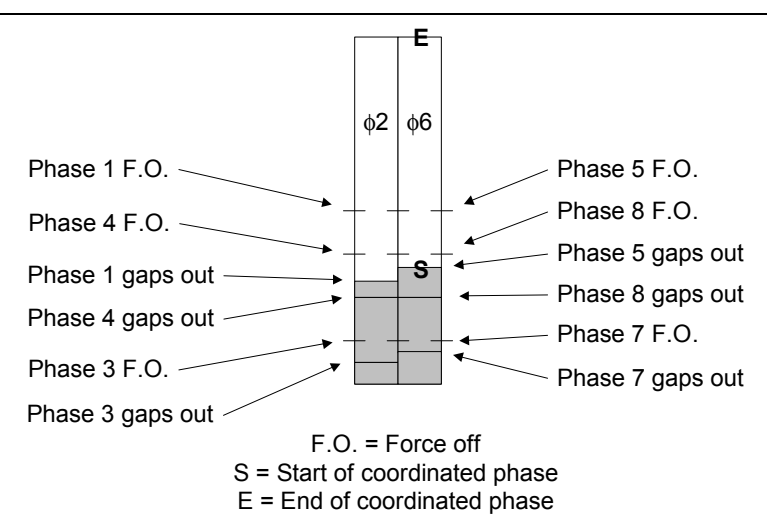

(b) Phases Gap Out

Figure 2-16 Coordinated Ring Structure

\subsubsection{Early Return to Green}

One of the problems with coordinating actuated controllers is that phases terminate before their force off times are reached due to light traffic demand, as shown in Figure 2-16b. As a result the 
coordinated phases have an "early return to green." The problem with this early return to green is that it is not predictable and usually varies by time of day and by intersection. Figure $2-17$ a shows a 4 intersection corridor where the offsets are timed so that smooth progression is obtained when their is no early return to green (Figure 2-16a). However, in practice there are varying levels of early return to green at the first three intersections (Figure 2-17b). This results in relatively poor progression because the traffic starting early at the first, must stop at the second. Similarly, traffic starting early at the third intersection must stop at the fourth intersection..

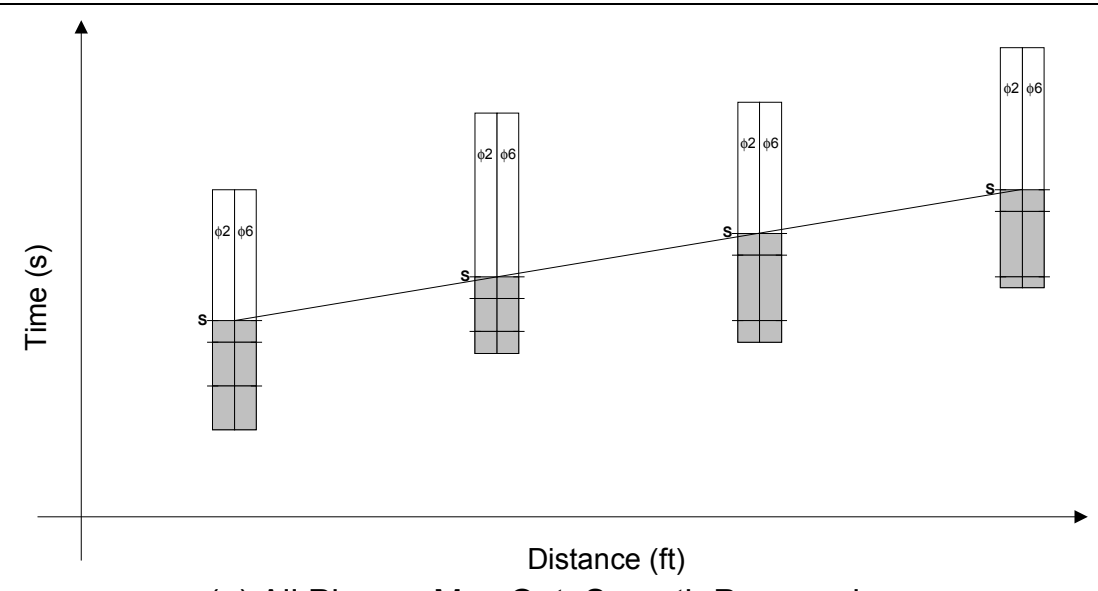

(a) All Phases Max Out, Smooth Progression

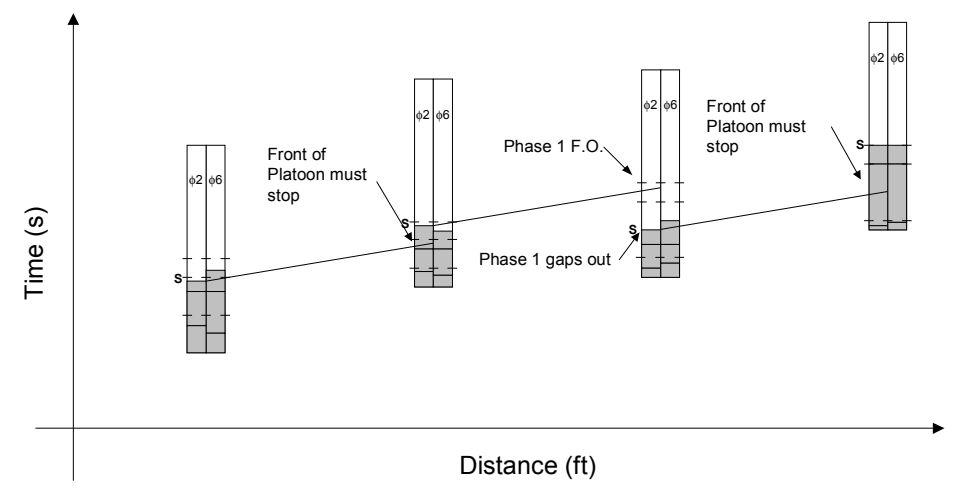

(b) Early Return to Green, Disrupted Progression

Figure 2-17 Time Space Diagrams

\subsubsection{Offsets}

Offsets are programmed to facilitate progression through a series of traffic signals. If the intersections are evenly spaced, as shown in Figure 2-18a, progression is possible in the opposing directions of travel. However, if the intersections are not evenly spaced, as shown in Figure 2-18b, good progression can usually only be achieved in one direction. Therefore, the 
direction with the heaviest traffic flow should receive the progression. Offsets can be referenced to either end of the coordinated green phase or latest start of the coordinated green phase.

Before actuated controllers were designed, the offset in fixed time controllers was primarily referenced to the start of the coordinated green phase. When actuated controllers were introduced, the only predictable point in the cycle was the end of the coordinated green phase (Figure 2-16, Point E) and this was used as the reference point. However, this was not very intuitive. Most recently actuated controller vendors have defined the offset relative to the latest start point of the coordinated green phase (Figure 2-16a, Point S) ${ }^{14}$. The latest start point is the point at which there is still enough time to serve the entire split of the coordinated phase. The offset is a very important parameter in determining the quality of progression along a corridor. Figure 2-19 shows how varying the offset impacts the travel time, delay, and percentage of stops along a segment of an arterial.

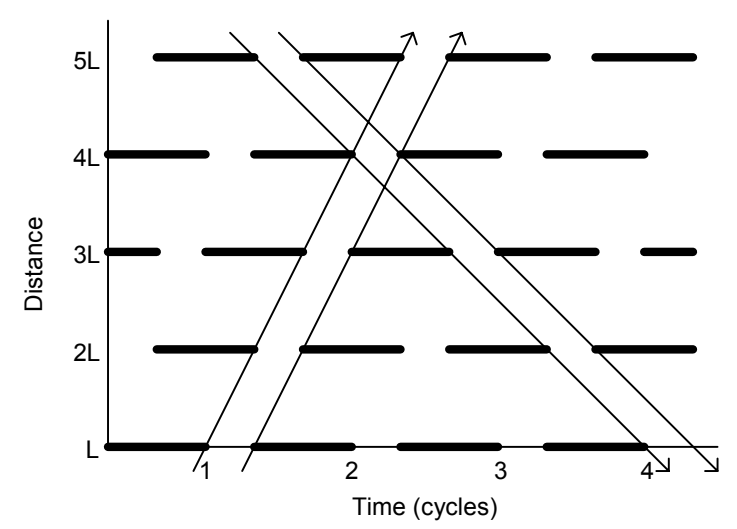

(a) Evenly Spaced Intersections

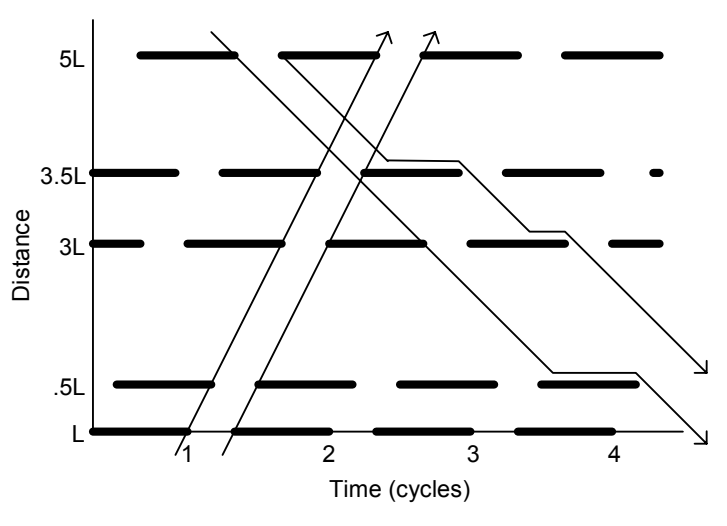

(b) Randomly Spaced Intersections

Figure 2-18 Effect of Intersection Spacing on Progression

\footnotetext{
${ }^{14}$ NEMA TS-2.
} 


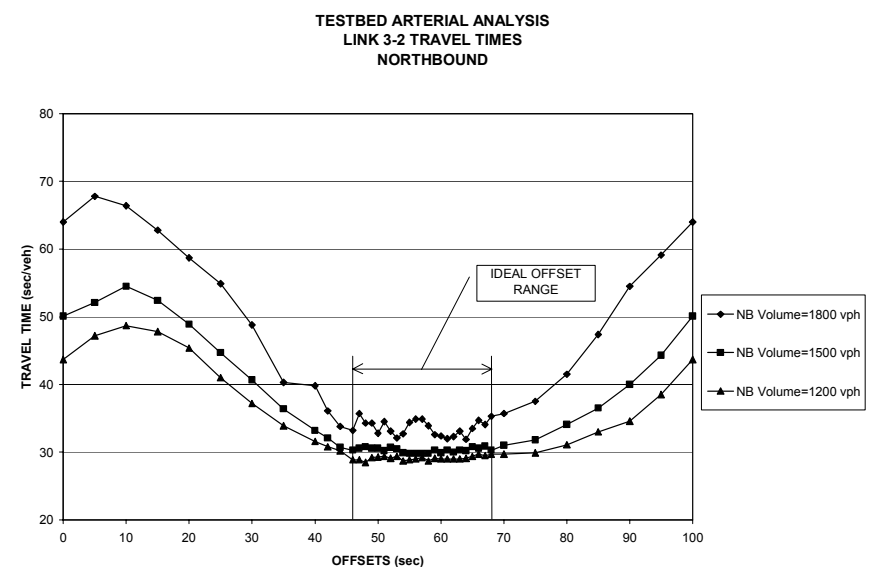

(a) Ideal Offsets based on Travel Time

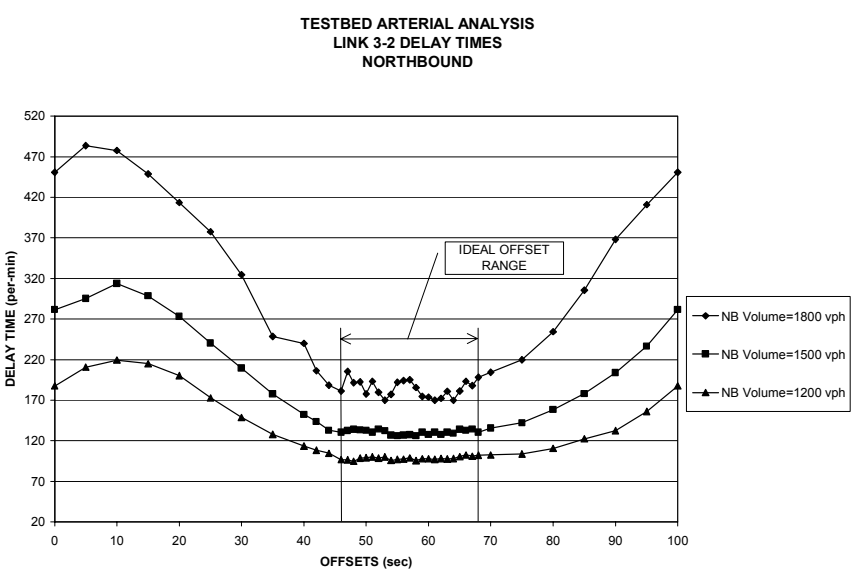

(b) Ideal Offsets based on Delay Time

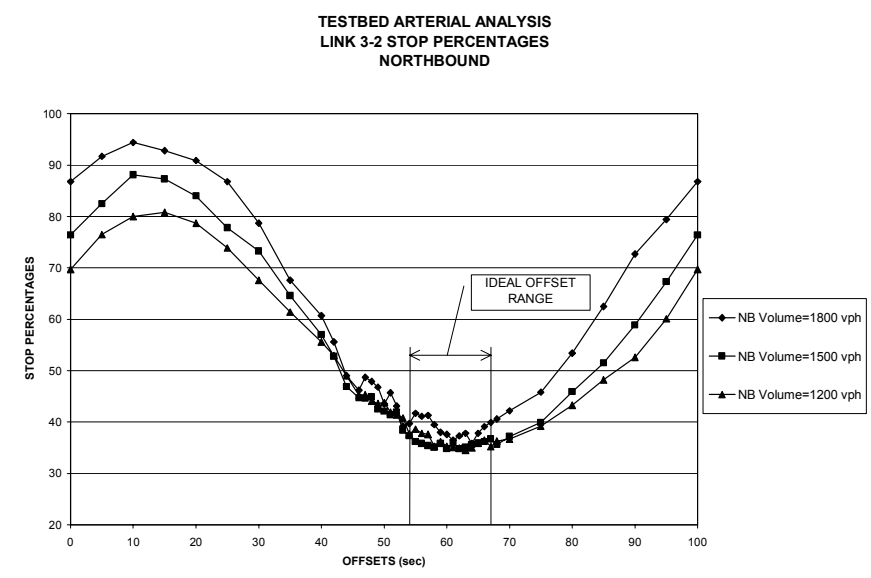

(c) Ideal Offsets based on Stop Percentages

Figure 2-19 Effects of Offsets 


\subsubsection{Designing Split, Cycle, and Offset}

The split, cycle, and offset values are designed using design hour volumes for each of the six time periods ( 5 coordination, 1 free) shown in Table 2-5. These volumes, along with intersection geometries, are input into the Synchro design package. Using engineering judgment, alternative phasing and sequence options are evaluated to determine the most appropriate split, cycle, and offset parameters for each of the six periods. 


\section{Chapter 3. Configuring Econolite Closed Loop Traffic Signal Systems}

As was discussed in Chapter 1, the numerical design of parameters, such as the phase times and coordination, is only part of the signal timing design. There are also a large number of vendorspecific communication and system level parameters that must be understood and configured. The purpose of Chapter 3 and Chapter 4 is to show how to implement the data tabulated in Chapter 2. This chapter will show, step-by-step, how to configure Econolite controllers to implement a closed loop system. Chapter 4 discusses how to configure Peek controllers to run a closed loop system.

After the design procedure in Chapter 2 has been completed, the next step is to enter this data into the controller and test it. The testing procedure is discussed in Chapter 5. The U.S. 231 system will continue to be used as an example, in particular, intersection 1. All U.S. 231 data can be found in Appendix B. Figure 3-1 shows the procedures that will be discussed in this chapter. Figure 3-2 shows the hardware-in-the-loop configuration used to evaluate the system being designed. 


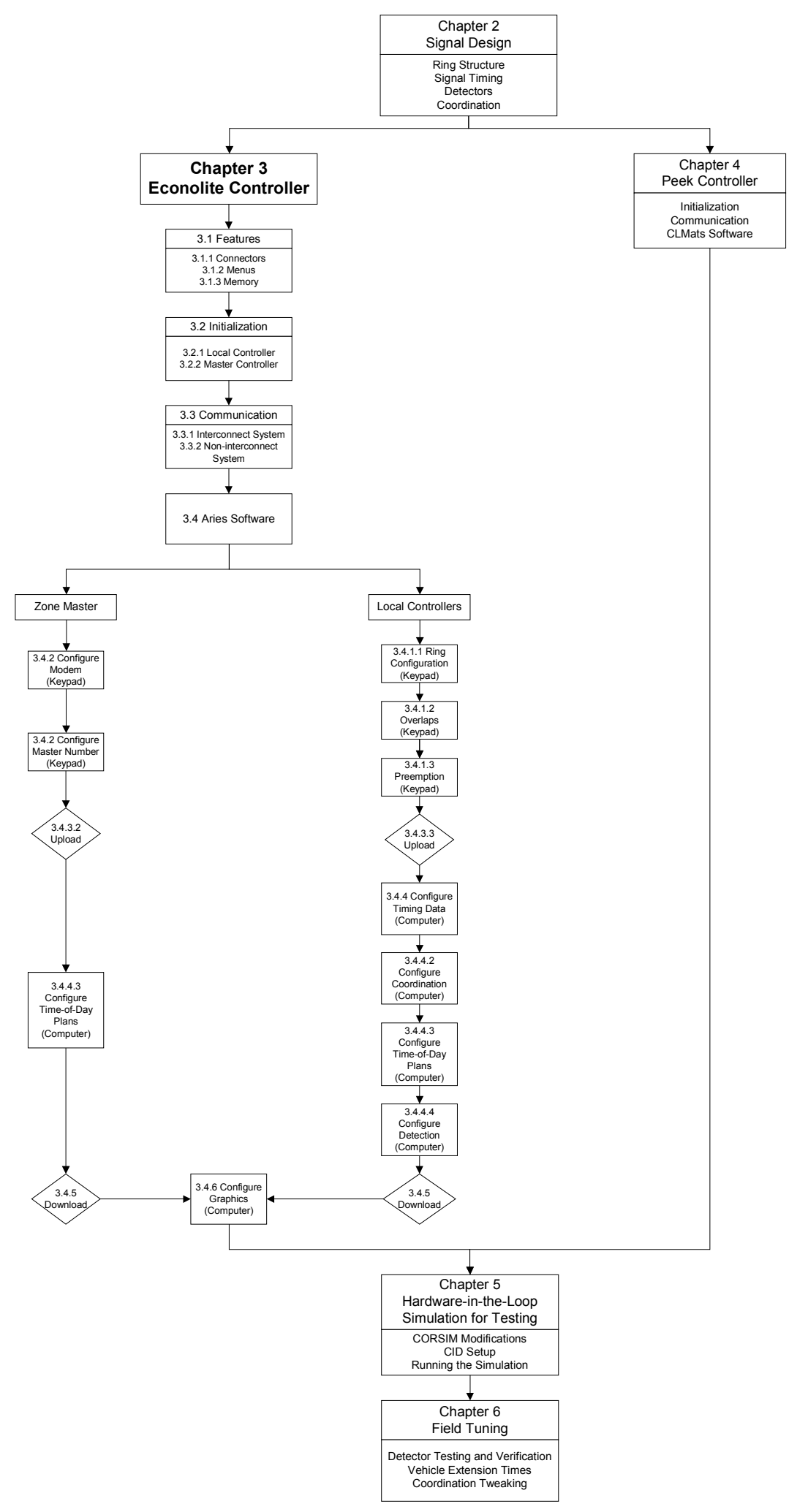

Figure 3-1 Chapter Concepts - Details of Configuring an Econolite System 


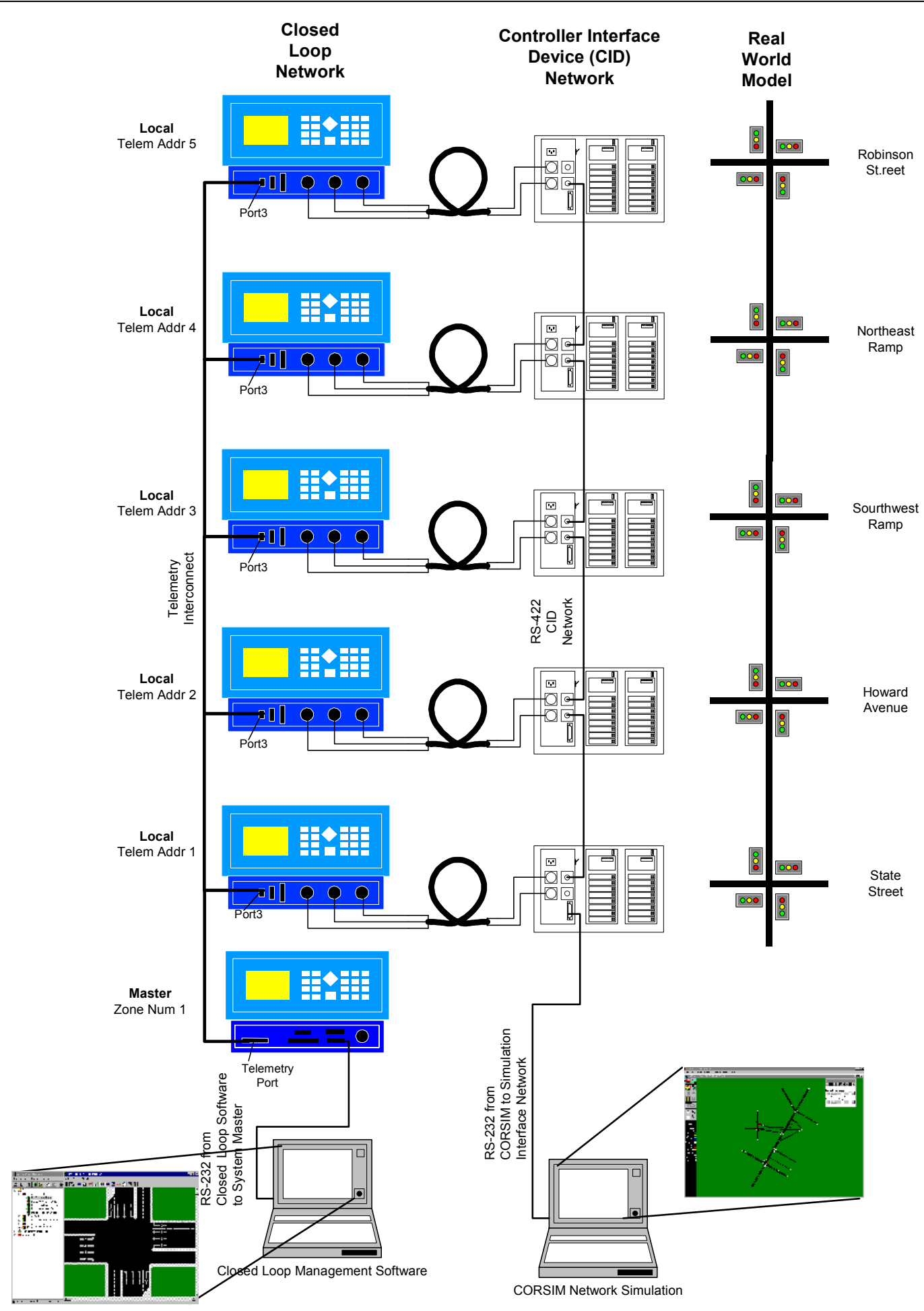

Figure 3-2 U.S. 231 Lab Setup 


\subsection{Controller Features}

\subsubsection{Connectors}

\subsubsection{Local Controller}

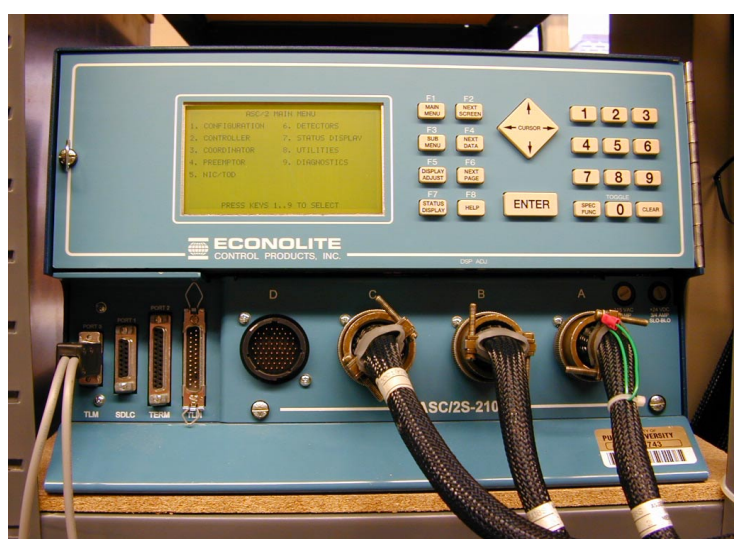

(a) Local Controller

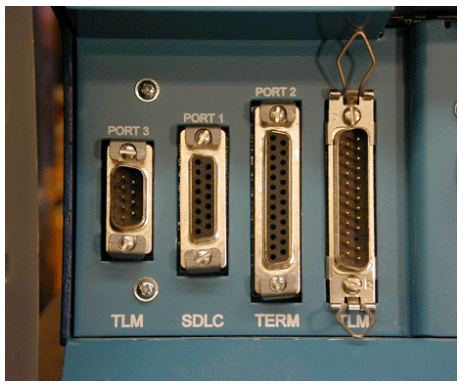

(b) Communication Ports

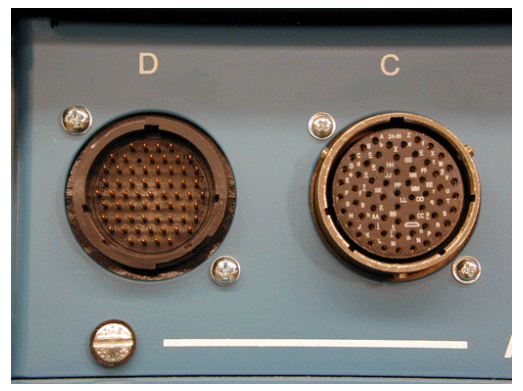

(c) NEMA D \& C Connectors

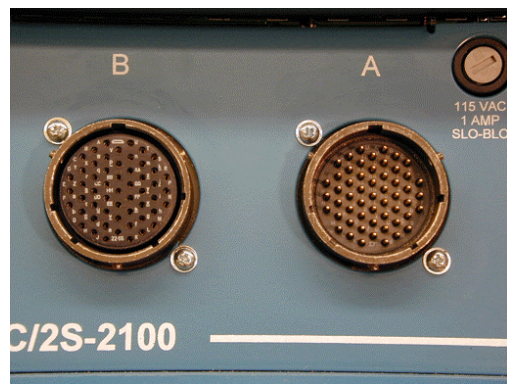

(d) NEMA B \& A Connectors

Figure 3-3 Local Controller and Connectors

The Econolite local controller shown in Figure 3-3a has 4 connectors and 4 ports on its front panel. The connectors provide an interface to the signal cabinet or a controller interface device (CID) in a lab setting as shown in Figure 3-2. Port 1, or the synchronous data link control (SDLC), is used with Type 1 controllers to interface with the cabinet. In Type 1 cabinets, the SDLC port is used instead of connectors A-D. Port 2 is used when directly connecting a computer or laptop to the controller to exchange data. When setting up an interconnected system with a master, port 3 is used to communicate with the master via a telemetry link as shown in Figure 3-2. Specific pin assignments for the connectors and ports can be found in Appendix B of the Econolite Controller Programming Manual. ${ }^{15}$

${ }^{15}$ Econolite Local Controller Programming Manual, Appendix B. 


\subsubsection{Zone Master}

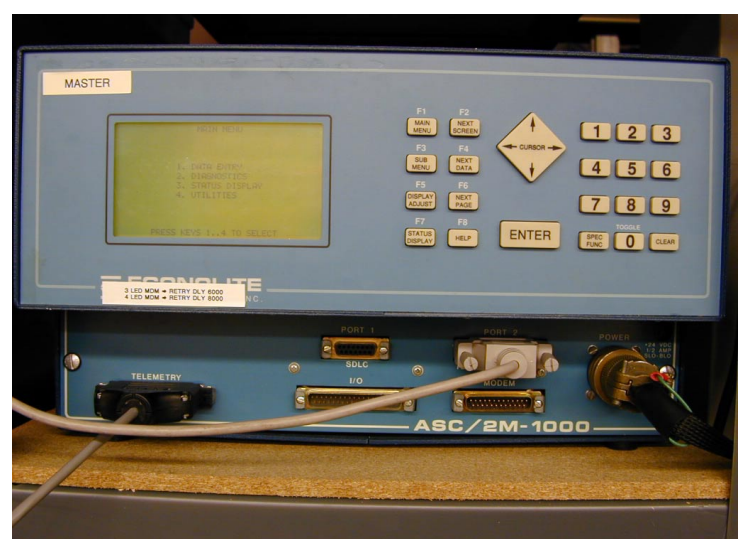

(a) Master Controller

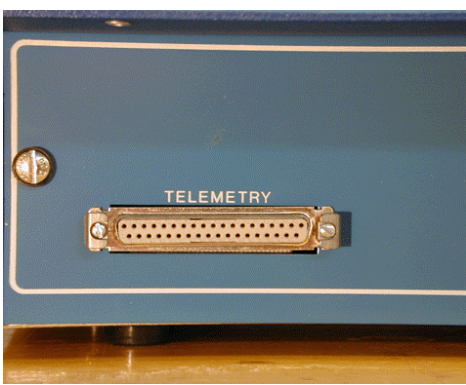

(b) FSK Modem Link to Local

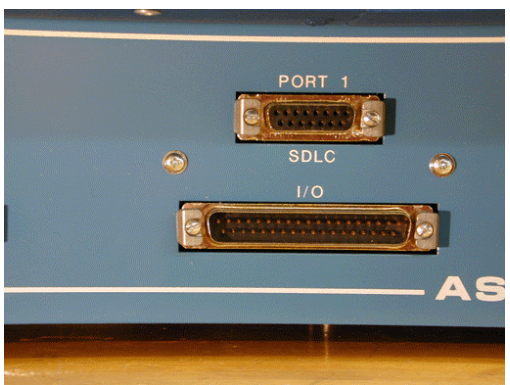

(c) SDLC and Cabinet Interface

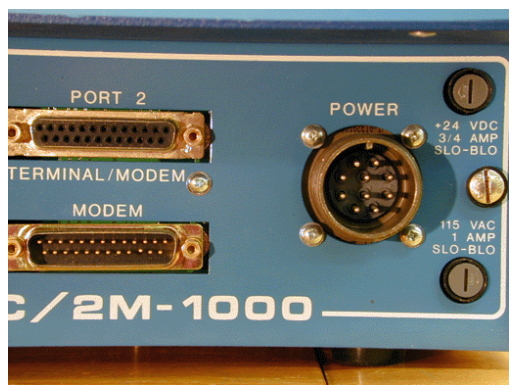

(d) Power and Direct Connect

Figure 3-4 Zone Master and Connectors

The Econolite zone master shown in Figure 3-4a has 1 connector and 5 ports on its front panel. Port 1 and the I/O port provide an interface to the cabinet. The telemetry port in Figure $3-4 \mathrm{~b}$ is used when interconnecting local controllers as shown in Figure 3-2. Port 2 is used when connecting directly to a computer or laptop. The modem port is used to exchange data over a telephone line with the Aries software. When the master is interconnected with the local controllers, data can be exchanged with the local controllers through the master, requiring only one telephone line installation - in the cabinet with the master. If data is exchanged by a direct laptop connection, this eliminates the need to go to each cabinet and directly connect to each local controller. Specific pin assignments for the connector and ports can be found in Appendix B of the Econolite Master Programming Manual. ${ }^{16}$

${ }^{16}$ Econolite Zone Master Programming Manual, Appendix B. 


\subsubsection{Menus}

All menus in the controller can be accessed from the main menu. It is very important to become familiar with the keys and how the menus are set up. Data has to be entered in different formats, including numerical, yes/no, and enabled/disabled. It is very important to always hit ENTER or cursor off of the last data entry before leaving a menu. The data will not be stored if a field is still active when the menu is exited. A hierarchy of the menu structure can be found in Appendix $D$ of the Econolite Controller Programming Manual. ${ }^{17}$

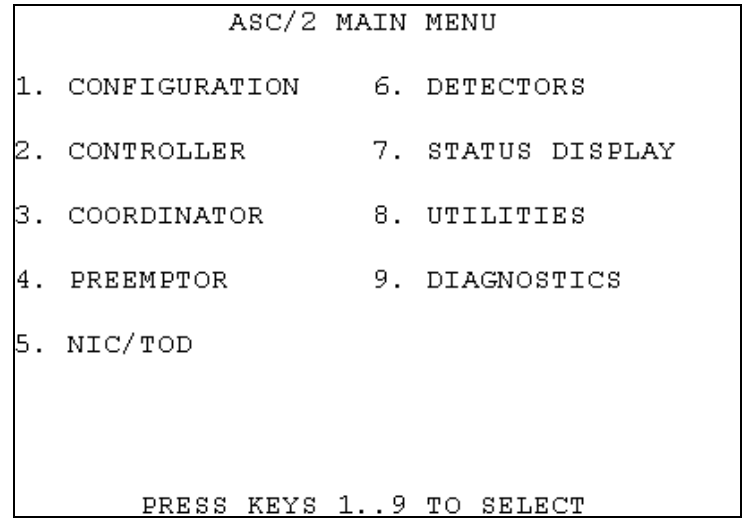

(a) Main Menu

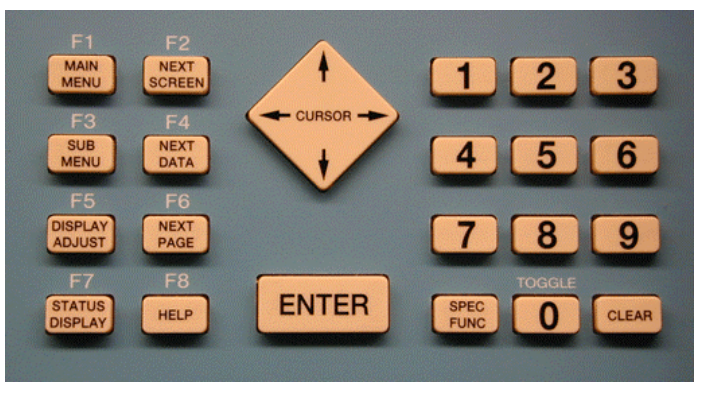

(b) Keyboard

Figure 3-5 Econolite Local Controller Main Menu and Keyboard

${ }^{17}$ Econolite Local Controller Programming Manual, Appendix D. 
Main Menu (F1)

Displays main menu, from which all submenus are accessed.

Next Screen (F2)

Press F2 followed by the direction key in a data entry screen will advance to the next screen in that direction, if one exists.

\section{Submenu (F3)}

Press F3 while in any data entry screen to return to the current submenu.

\section{Next Data (F4)}

Press F4 followed by the direction key in any data entry screen will advance to the next non-zero data field.

\section{Display Adjust (F5)}

Press F5 to adjust the display contrast. In some models, the display is adjusted using the dial under the keyboard.

\section{Next Page (F6)}

Press F6 followed by the direction key to advance to the next group of data entry screens in the current submenu.

\section{Status Display (F7)}

Press F7 to view the status of the intersection. (See below.)

\section{Help (F8)}

Press F8 in any menu or with the cursor in any data entry field to display help screens. Press F8 again to exit the help screens.

\section{Enter}

Always press the Enter key after entering data before exiting the menu.

\section{Cursor}

Use the cursor button to move the cursor in the desired direction and to scroll through data screens.

\section{Numbers (1-9)}

Press the number keys to access the corresponding menu and to enter data.

\section{Toggle (0)}

Press 0 to toggle yes/no, enabling $(X=$ on and blank $=$ off, and other data fields that have more than one preset parameter.

\section{Clear}

Press Clear to exit help screens and to erase the current data entry and restore the previous data entry.

\section{Special Function}

Press Spec Func then numbers 1-6 to enter the hexadecimal values $A-F$ in screens that require these numbers.

\section{Figure 3-6 Econolite Key Functions}

The intersection status display provides real-time information about the state of the controller:

1. The numbers 1 through 12 at the top of the screen represent phases 1 through 12.

2. $A$ ' $T$ ' in the $T / N$ row indicates the phases that are currently timing. An ' $N$ ' in this row indicates the phases that are going to be timed next.

3. A ' $C$ ' in the VEH row indicates a call from a vehicle detector. $A n$ ' $R$ ' in this row indicates that the particular phase is in recall, probably because it has no detection. An ' $N$ ' indicates that the phase is non-actuated. For testing purposes, vehicle calls can be placed manually by pressing the number key corresponding to the desired phase (1-8).

To place calls on phases 9-12, use the cursor keys: $\uparrow(9), \rightarrow(10), \downarrow(11), \leftarrow(12)$.

4. $A$ ' $C$ ' in the PED row indicates a call from a pedestrian detector. Likewise, an ' $R$ ' in this row indicates that the pedestrian phase is in recall. An ' $N$ ' in this row indicates the pedestrian phase is non-actuated. Pedestrian calls can be placed manually by pressing the Special Function key followed by the number key for the desired phase. 
5. The current phases are also indicated on the left side of the screen with the timing interval, termination indication, time remaining, and pedestrian status. Timing intervals that can be shown include MGRN (minimum green), MGRN DONE, GRN HOLD, GRN REST, VEXT (vehicle extension), GRN XFER (green transfer), YEL (yellow), RED, RED REST, RED XFER, and HOLD. Termination indications are displayed to indicate the reason for ending the green interval and include PREEMPT, FORCE OFF, MAN ADV (manual advance), MAX OUT, GAP OUT, MIN GAP, and GUAR PASS (guaranteed passage). Pedestrian intervals that are shown include WALK, WALK REST, WALK HOLD, and PCLR (pedestrian clearance).

6. The intersection coordination status is shown below the phase status. CMD SRC indicates whether the controller is coordinated through telemetry (TLM), hardwire (HDW), or not-interconnected (NIC). The coordination pattern that is being run is shown next to PTN. The local cycle timing is shown next to LOC CYC. The system cycle timing is shown next to SYS CYC if a master is being used. When running coordination, these two times will not always coincide. The master cycle is based on the cycle length entered in the system parameters menu. The local cycle is based on the cycle length for the selected coordination pattern. The two might not be the same, especially if intersections are half-cycled or a non-zero offset is used. COORD indicates that the controller is in coordination with the system. If it is not in coordination, the method of offset correction is shown as ADD, DWELL, or SUB. Offset correction is discussed later in this chapter.

7. If preemption is being used, the call status is shown in the bottom left corner of the status display screen for priority preemptors 1-6 and bus preemptors 1-4. An ' $A$ ' indicates the preemptor is active, a ' $C$ ' indicates a call is present, $a$ ' $D$ ' indicates the preemption delay is timing, and an ' $l$ ' indicates that the inhibit timer is timing. For testing purposes, preemption calls can be placed manually by pressing ENTER then the corresponding keyboard number (priority $1-6$ ) or cursor (bus $\uparrow 1, \rightarrow 2, \downarrow 3, \leftarrow 4$ ).

8. The NIC program step number and pattern called by that step are shown in the bottom right corner along with the TOD program step number and the current date and time. 


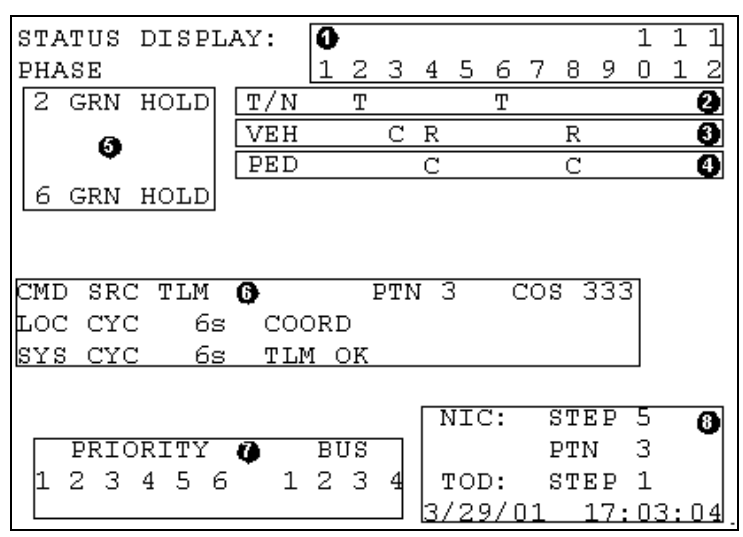

Figure 3-7 Status Display (F7)

\subsubsection{Memory}

The Econolite controller stores information on an EEPROM that can only be removed when the controller is turned off. Removing or replacing an EEPROM while the controller is still on can erase or damage the EEPROM. The controller cannot run without an EEPROM. Figure 3-8 shows an Econolite EEPROM. If a controller needs to be replaced, removing the memory module from the old one and inserting it into the new controller is an easy way to transfer all configuration parameters.

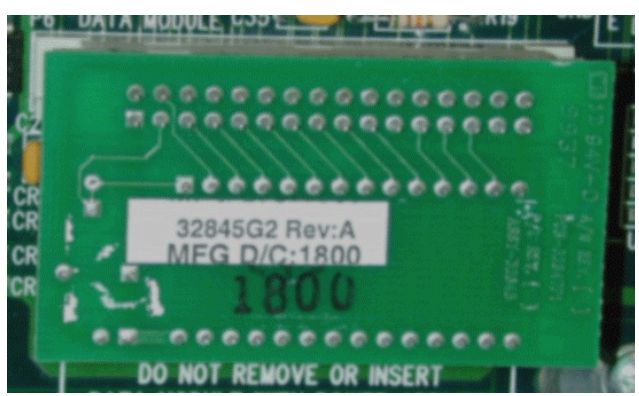

(a) Back

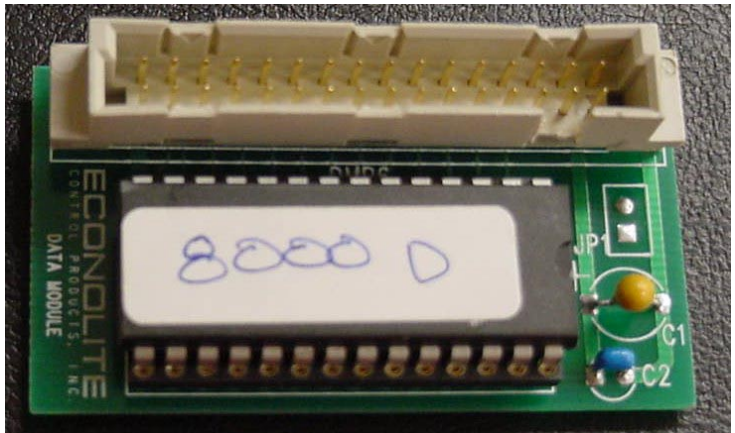

(b) Front

Figure 3-8 Econolite EEPROM 


\subsection{Initializing the Controller}

Prior to programming the controller, it is very important to erase unwanted information that may exist in the controller. Not clearing the memory can lead to many problems in the future. For example, irrelevant time-of-day programs could be entered or unused detectors could be enabled. Troubleshooting will also be simplified by initializing the controller because the programmer always starts from the same default values.

\subsubsection{Local Procedure:}

Main Menu $\rightarrow$ 8. Utilities $\rightarrow$ 1. Copy $\rightarrow 3$. Backup Timing

The quickest way to clear the memory and start with a valid timing plan is to copy the backup timing plan. This procedure is done in one step. The controller comes with a basic timing plan with predetermined parameters. These values will be placed in the controller timing data menu. Also, data in the submenus listed below will be cleared.

- Submenu 3 Coordinator data

- Submenu 4 Preemption data

- Submenu 5 NIC/TOD data

- Submenu 6 Detector data

- Submenu 9 Diagnostic Enables.

Please note that not all data in the controller can be cleared. Data in the submenus not listed (such as telemetry configuration and controller timing parameters) might be a starting point when troubleshooting the controller. 


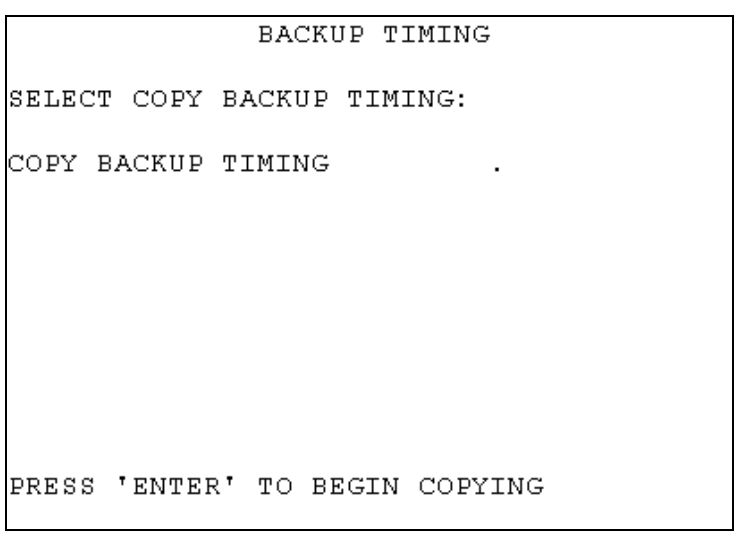

(a) Local Controller Copy Backup (MM, 8, 1, 3)

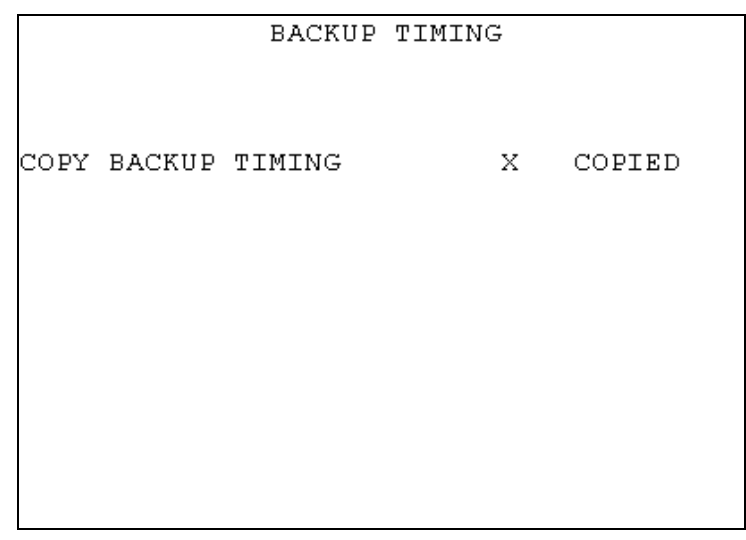

(b) Copy Backup Confirm

Figure 3-9 Initialization: Local Controller Copy Backup Timing

\section{Main Menu $\rightarrow$ 8. Utilities $\rightarrow$ 2. Memory Clear}

Another method of clearing the memory is to individually clear the submenus listed above. This will not copy any default timing data, only clear those submenus. Move the cursor down to Coordinator Data and press ' $O$ ' to toggle an ' $X$ '. This marks the data in this submenu to be cleared. Select other data to be cleared. After all have been marked, press ENTER. The data clear is confirmed as shown in Figure 3-10b.

\begin{tabular}{|c|c|}
\hline & MEMORY CLEAR \\
\hline SELECT & DATA TO CLEAR: \\
\hline CLEAR & COORDINATOR DATA \\
\hline CLEAR I & NIC/TOD DATA \\
\hline CLEAR & PREEMET DATA \\
\hline CLEAR & DETECTOR DATA \\
\hline CLEAR & DIAGNOSTIC ENABLES \\
\hline PRESG & ${ }^{\top}$ ENTER $^{\top}$ TO BEGIN CLEARING \\
\hline
\end{tabular}

(a) Memory Clear Menu (MM, 8, 2)

\begin{tabular}{|llll|}
\hline \multicolumn{3}{|c|}{ MEMORY CLEAR } & \\
& & & \\
CLEAR COORDINATOR DATA & $\mathrm{X}$ & CLEARED \\
CLEAR NIC/TOD DATA & $\mathrm{X}$ & CLEARED \\
CLEAR PREEMTT DATA & $\mathrm{X}$ & CLEARED \\
CLEAR DETECTOR DATA & $\mathrm{X}$ & CLEARED \\
CLEAR DIAGNOSTIC ENAELES & $\mathrm{X}$ & CLEARED \\
& & & \\
& & & \\
& & & \\
& & & \\
\end{tabular}

(b) Memory Clear Confirmation

Figure 3-10 Initialization: Local Controller Memory Clear 


\subsubsection{Master Procedure:}

The zone master memory cannot be cleared. However, default data screens are provided in the Econolite Programming Manual ${ }^{18}$. If the master has been previously used in other systems, it is recommended to program the default data as a starting point.

${ }^{18}$ Econolite Zone Master Programming Manual, Appendix A 


\subsection{Setting up Communication}

\subsubsection{Interconnected System with Master}

Once the master and local controllers are initialized, communication between them should be established. Make sure all local controllers are connected to the master with the appropriate telemetry cable. The cable should be plugged into Port 3 on the local controllers and the Telemetry port on the Master.

\subsubsection{Telemetry Configuration}

\subsection{Local Procedure:}

Main Menu $\rightarrow$ 1. Configuration $\rightarrow 5$. Port 2

In the Configuration submenu of each local controller, Port 2 should be configured as shown in Figure 3-11. Port 2 is the communications terminal that can allow a computer or laptop to be directly connected to the controller. Port 2 is not often used if computer communication will take place through the master, however it should be enabled for possible use. The protocol should be set to terminal and enabled. The data rate should be 19,200 bits per second and the data, parity, stop should be $8, \mathrm{~N}, 1$.

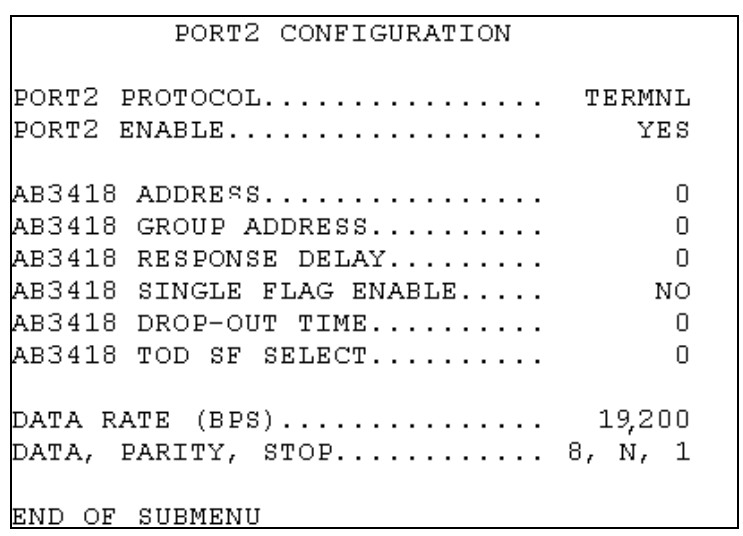

Figure 3-11 Communication: Local Controller Port 2 Configuration (MM, 1, 5)

Main Menu $\rightarrow$ 1. Configuration $\rightarrow 6$. Port 3

In the Configuration submenu of each local controller, Port 3 needs to be configured. The protocol should be TELEM for telemetry and enabled. Each controller needs to be assigned a 
unique telemetry address. Since the example intersection is the first in the system, it is assigned address 1 . Valid telemetry addresses are 0-24. The telemetry response delay should be set based on the type of telemetry module in the controller. Without powering down the system, open the front panel of the controller and look at the telemetry module.

- If the module has 4 LEDs, as shown in Figure 3-12c, the delay should be set to 8000 .

- If the module has 3 LEDs, as shown in Figure 3-12d, the delay should be set to 6000 . The AB3418 data is used for California protocols and is not used in Indiana. All data entries should be zero or no. In the second screen (page down), set the port to full duplex, and data rate to 1200 bps for twisted pair (TDM/FSK telemetry module) and 9600 or 19,200 bps for fiber optic (RS-232 telemetry module). The data, parity and stop should be $8,0,1$. Once the controllers have been enabled on the master, communication can be confirmed on the status display screen.

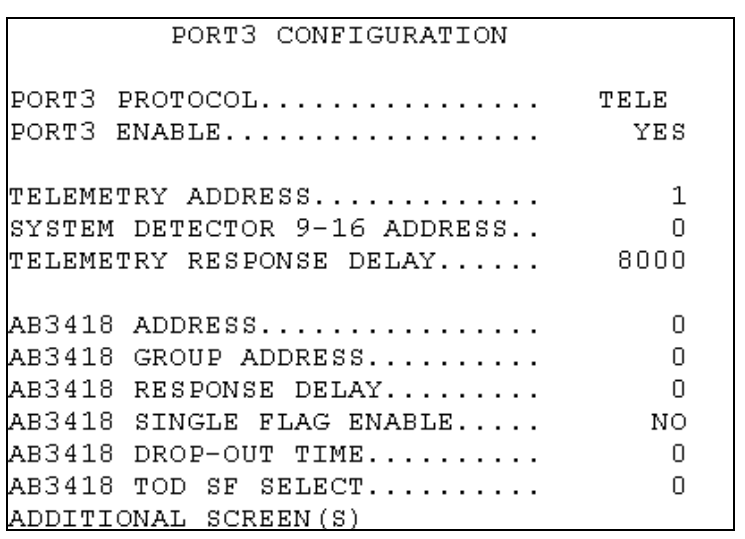

(a) Port 3 Configuration Screen 1 (MM, 1, 6)

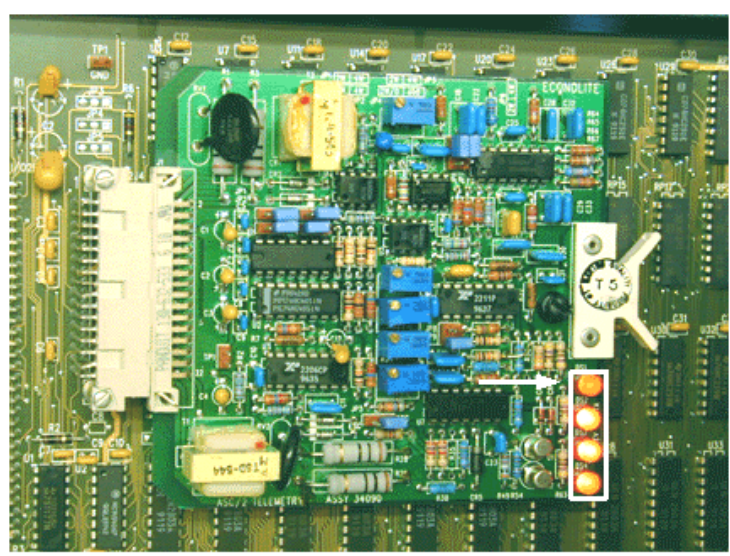

(c) 4 LED Module $\rightarrow$ TRD $=8000$

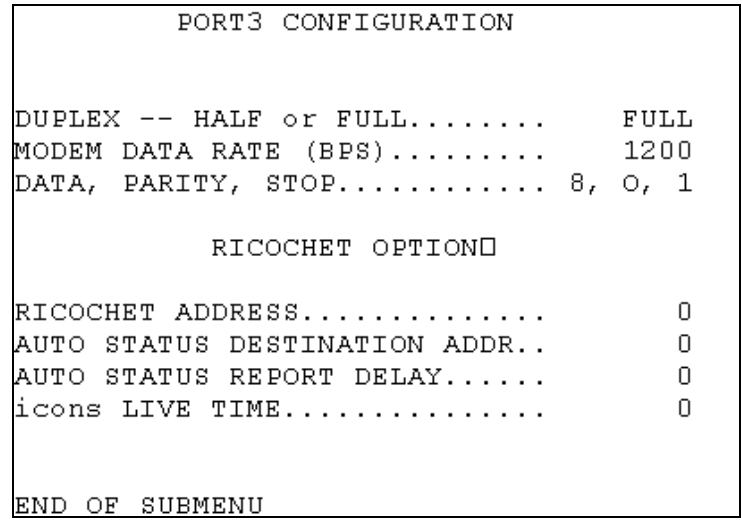

(b) Port 3 Configuration (MM, 1, 6, PgDn)

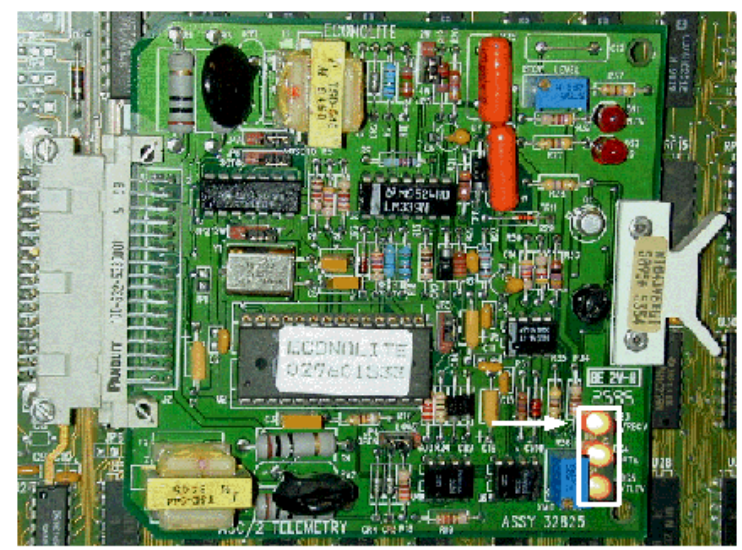

(d) 3 LED Module $\rightarrow$ TRD $=6000$

Figure 3-12 Communication: Local Controller Port 3 Configuration 


\subsection{Master Procedure:}

Main Menu $\rightarrow$ 1. Data Entry $\rightarrow 0$. Next Menu $\rightarrow 2$. Enable Devices

The master will also have to be assigned a telemetry number, but this will be done in Section 3.4.2.2. After Port 3 has been configured on all local controllers, the controllers have to be enabled on the master. In the enable devices screen, local controllers are enabled by placing an ' $X$ ' in the second column under the controller heading. The numbering should coincide with the local telemetry address assigned. For this system, the local controllers were assigned telemetry addresses 1-5. Devices 1-5 have been enabled in the controller column as shown in Figure 3-13a. Telemetry channel 1 is enabled. If system detectors will be used, they should be enabled here.

\section{Main Menu $\rightarrow$ 1. Data Entry $\rightarrow$ 0. Next Menu $\rightarrow$ 3. Telemetry Sequence Channel 1}

After the controllers are enabled, they need to be assigned telemetry channel 1 . In the first column under CTR (controller), the controller number from the Enable Devices screen must be entered next to the telemetry address used for each controller. The whole process is easiest if intersection 1 is enabled as controller 1 and assigned to telemetry address 1 , and each subsequent intersection follow the same pattern.

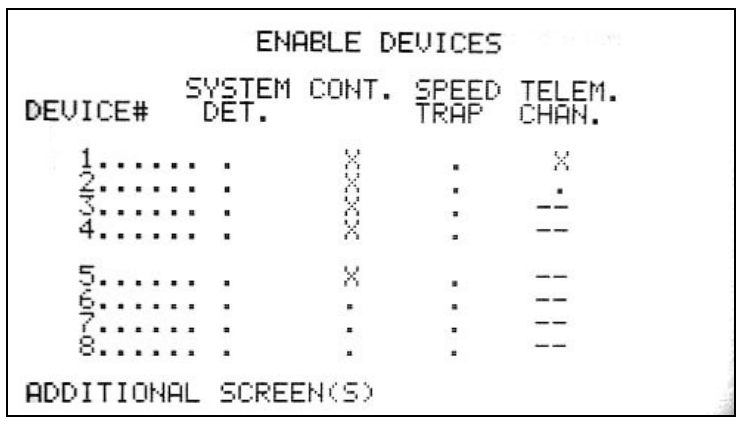

(a) Enable Devices Screen (MM, 1, 0, 2)

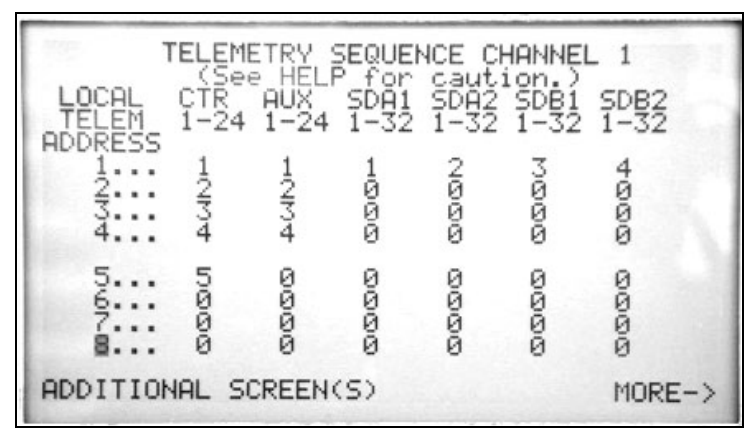

(b) Telemetry Sequence Ch $1(\mathrm{MM}, 1,0,3)$

Figure 3-13 Communication: Master Telemetry Channel Assignments

After a system reaches 12 intersections or 24 requests, a second telemetry channel should be added to the master. Requests are defined in Table 3-1. For example, a controller with eight system detectors and one speed trap will equal seven requests. A telemetry channel can handle 40 requests, but the read back times will go from 1 per second at 24 requests or less to 1 every 1.5 seconds at 40 requests. 
Table 3-1 Master Telemetry Channel Requests

\begin{tabular}{|c|c|c|}
\hline Parameter & Master Abbreviation & Requests \\
\hline Controller & CTR & 1 \\
\hline Auxiliary 1 & AUX1 & 1 \\
\hline System Detectors 1 \& 2 & SDA1 \& SDA2 & 1 \\
\hline System Detectors 3 \& 4 & SDB1 \& SDB2 & 1 \\
\hline System Detectors 5 \& & SDC1 \& SDC2 & 1 \\
\hline System Detectors 7 \& 8 & SDD1 \& SDD2 & 1 \\
\hline Speed Trap 1 & STA & 1 \\
\hline Speed Trap 2 & STB & 1 \\
\hline Auxiliary 2 & AUX2 & 1 \\
\hline
\end{tabular}

Main Menu $\rightarrow$ 1. Data Entry $\rightarrow 0$. Next Screen $\rightarrow$ 9. Telemetry Configuration

The telemetry channels that are used also need to be configured. The data rate should be 1200 bits per second for the twisted pair connection and 9600 or 19,200 for fiber optic. The bits, parity, and stop should be $8,0,1$. The other values can be left at the default.

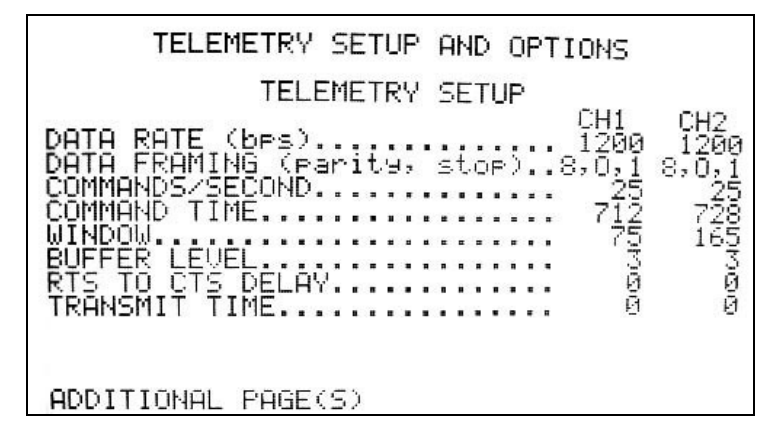

Figure 3-14 Communication: Master Telemetry Channel Configuration

Main Menu $\rightarrow$ 1. Data Entry $\rightarrow 0$. Next Screen $\rightarrow 6$. Configuration

In order to access the Master in the field with Aries, the modem must be configured. In the configuration screen, the communication type should be modem and other parameters are specific to the modem used. If Aries is used with a direct connection, Port 2 should be selected and configured. The speed should be 19,200 bps and the data format 7, E, 1. The increased speed is possible with a direct connection but not with a modem. The data transfer between Aries and the master controller will be much faster, however the transfer to the local controllers will only be as fast as the telemetry connection. 


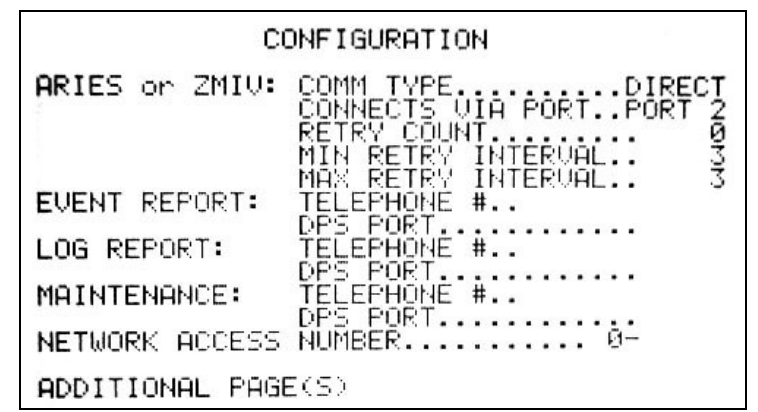

(a) Modem Configuration (MM, 1, 0, 6)

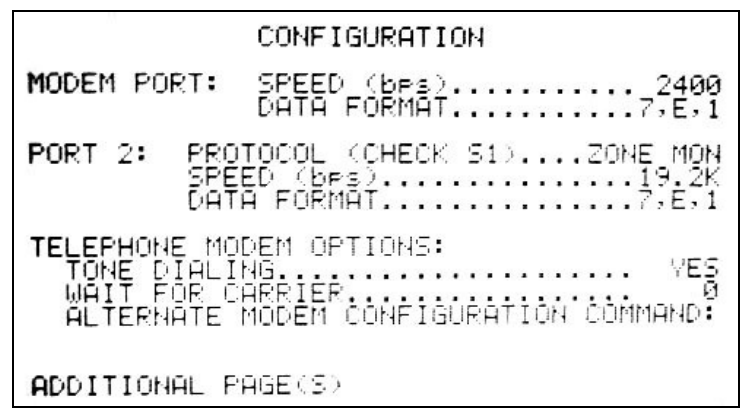

(b)Modem Configuration (MM, 1, 0, 6, PgDn)

Figure 3-15 Communication: Master Modem Configuration

\subsubsection{Telemetry Verification}

\subsection{Master Procedure:}

Main Menu $\rightarrow$ 2. Diagnostics $\rightarrow 2$. Controller

Communication can be verified from the controller diagnostics screen. The status of enabled controllers show telemetry ok, as shown in Figure 3-16a. If telemetry fails for one of the controllers, check the local controller telemetry address and make sure it corresponds to the controller that was enabled on the master. If two controllers in the system are configured with the same address, this could also cause telemetry to fail. An unconnected cable can also cause telemetry to fail.

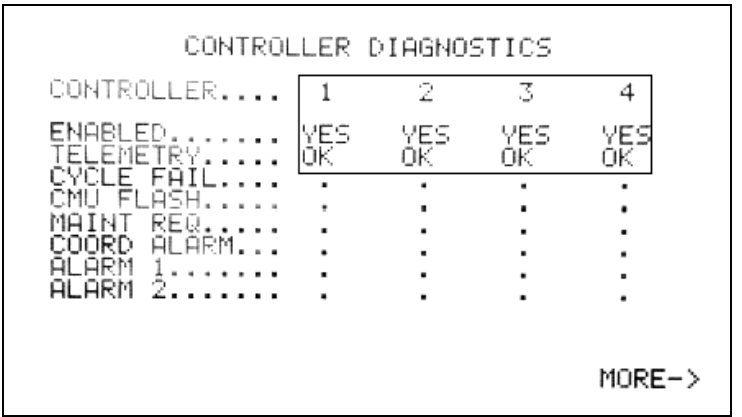

(a) Controller Telemetry Status (MM, 2, 2)

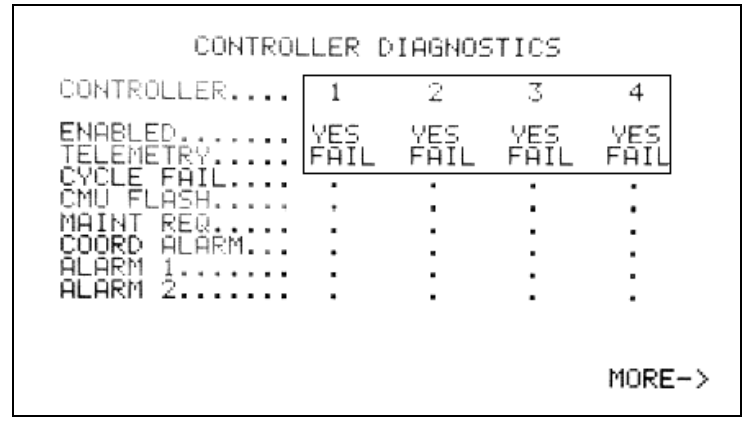

(b) Controller Telemetry Status Failed

Figure 3-16 Communication: Master Telemetry Verification 


\subsection{Local Procedure:}

\section{F7. Status Display}

Verifying telemetry is quickest and easiest using the master because the state of the whole system can be viewed in one screen. However, the telemetry can be also be verified in each local controller. The quickest way to confirm communication is on the status display screen. If TLM OK appears next to the system cycle, as shown in Figure 3-17a, communication is ok. If TLM ERR appears, as in Figure 3-17b, there is something wrong with either the master configuration, local configuration, or the communication infrastructure.

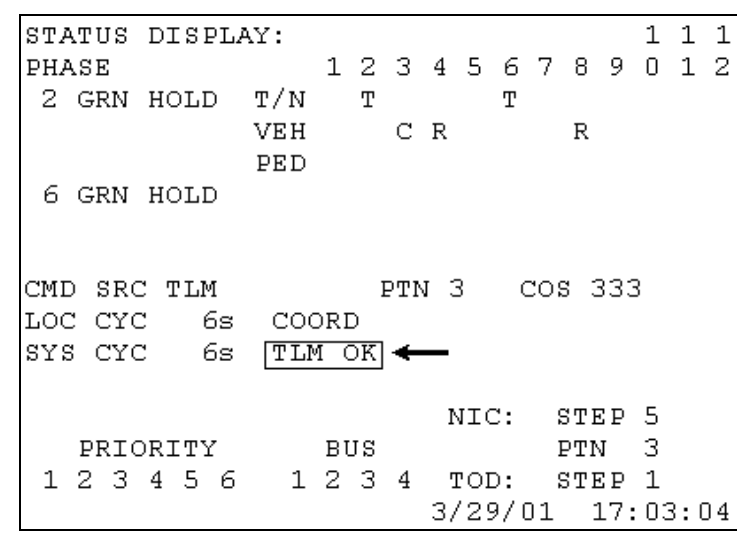

(a) Local Status Display Telemetry OK

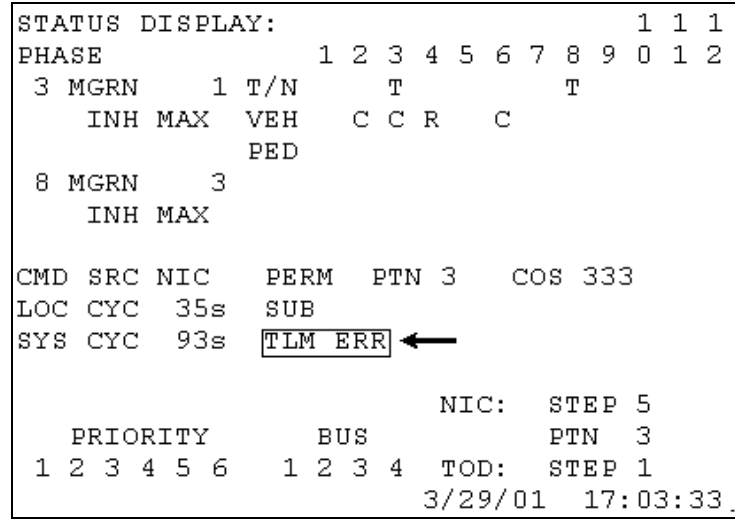

(b) Local Status Display Telemetry Error

Figure 3-17 Communication: Local Telemetry Verification (F7)

\subsubsection{Interconnected System Time Clock}

\subsection{Master Procedure:}

Main Menu $\rightarrow$ 1. Data Entry $\rightarrow 1$. System Parameters

One of the many advantages of interconnected systems is clock synchronization. The master can set all local controller time clocks. This is very important for coordinated systems. Without the same time references, the controller offsets are useless. To set the clock, go to the data entry submenu shown in Figure 3-18a. The first system parameters screen allows the user to set the time and date. Enter must be pressed in order for the changes to take effect. This time will be downloaded to all local controllers. Also, the master can regularly synchronize the time of all local controllers. This feature is enabled in the fifth screen in the system parameters shown in Figure 3-18b by toggling "NIC Time Sync Enable. “ 


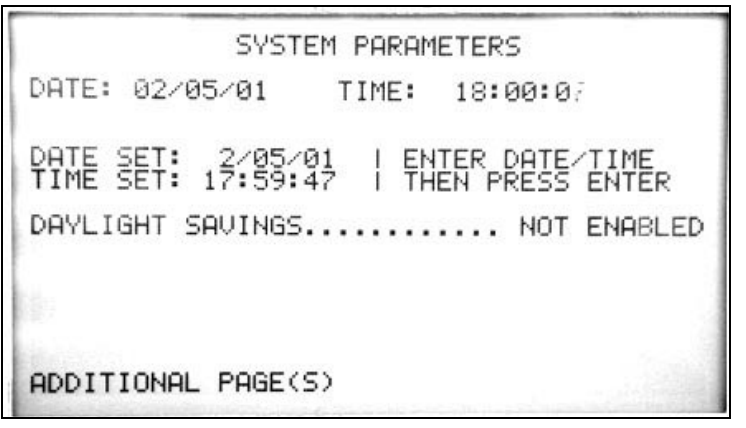

(a) Time and Date Configuration (MM, 1, 1)

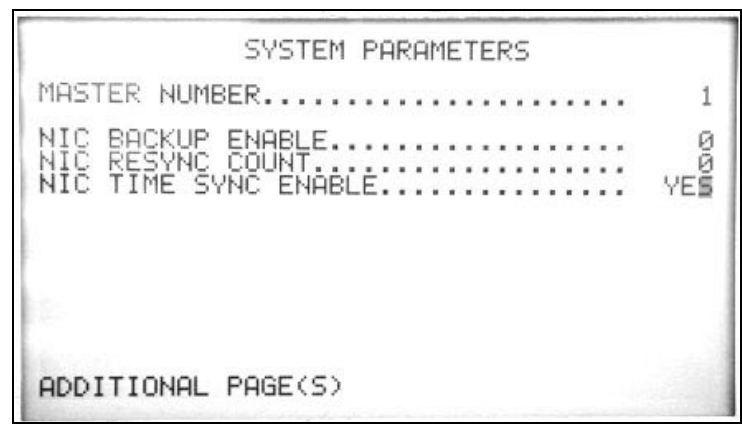

(b) NIC Sync Enable (MM, 1, 1, PgDn(4))

Figure 3-18 Master Time Configuration

\subsection{Local Procedure:}

Main Menu $\rightarrow$ 5. NIC/TOD $\rightarrow$ 1. Clock/Calendar

All local controllers in the system must have the same sync reference time and source. The source should be set to reference time and the default value of 0:00 can be used for the reference time. Make sure that all controllers in the system have the same sync reference time programmed. The other two sync sources occur at certain points in the master cycle, rather than on the time clock.

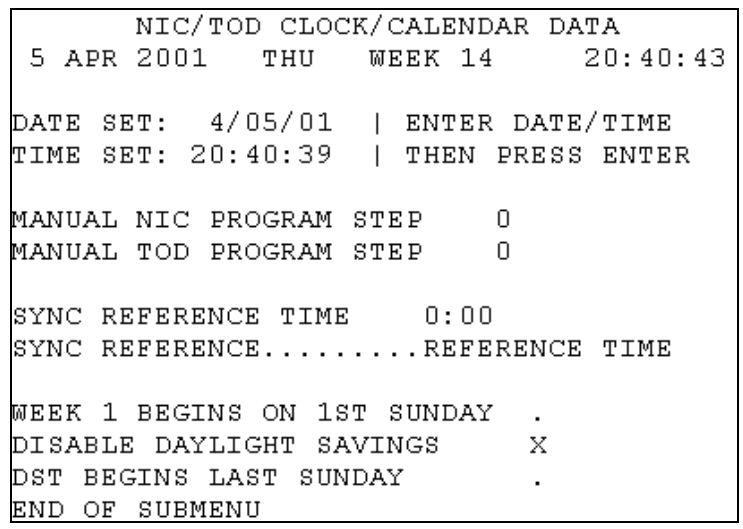

Figure 3-19 Local Controller Time and Date Configuration (MM, 5, 1) 


\subsubsection{Non-interconnected System}

In general, an interconnected system is preferred because it is easier to ensure synchronization. However, situations arise where it is not practical to deploy communication infrastructure to interconnect traffic signal equipment. In those cases, it is critical that all controllers be configured to reference the identical time. When programming the time clocks, extra care must be taken to program the clocks to the nearest second. A device such as a handheld Global Positioning System (GPS) receiver is usually the most precise and accurate time reference to use for this procedure.

\subsubsection{Local Procedure:}

Main Menu $\rightarrow$ 5. NIC/TOD $\rightarrow$ 1. Clock/Calendar

If a system with multiple controllers is running coordination plans, the time clock in each controller needs to be set. The clock in each controller needs to be as accurate as possible for optimal performance. Time clocks in the controllers will actually drift over time. Controllers in the field should be reset every year or after power outages to ensure synchronization. To set the time clock, go to the Clock/Calendar screen in the NIC/TOD submenu. Enter must be pressed for the changes to take effect.

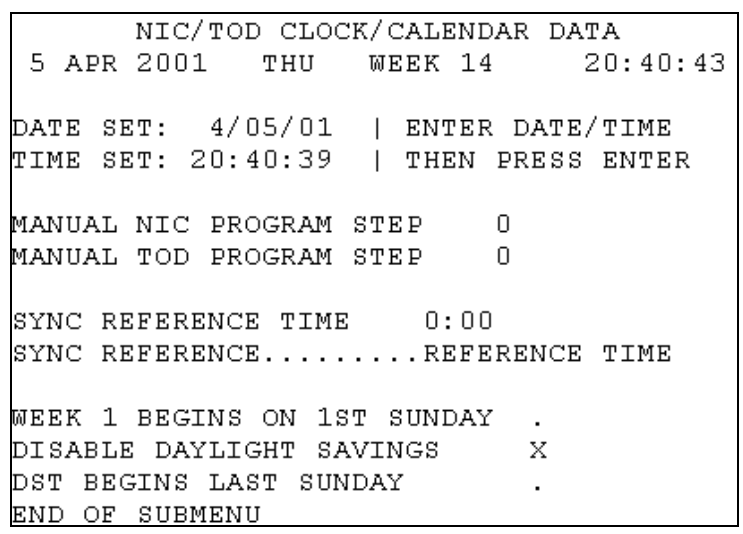

Figure 3-20 Local Controller Time and Date Configuration (MM, 5, 1) 


\subsection{Aries: Computer Interface}

Once the communication has been established, assuming all controllers are linked via telemetry, the configuration of the system can be performed using the Aries Management Software.

The Aries software has many benefits besides simplifying controller data entry. The typical sequence followed when using Aries is to upload the current controller data to the computer, modify it, and download the new data to the controller. Before the data is downloaded to the controller, it is saved on the computer. This backup copy is always available in case something happens to the controller and the data is erased. Instead of keying in all of the data by hand, which can take hours, the data is simply downloaded and the intersection is running in a few minutes. Furthermore, with the modem plugged into a phone line, new timing plans can be entered into Aries and downloaded to the controllers without leaving the office, although this practice is discouraged by INDOT to prevent the user from downloading undesired data that could affect the safety of the intersection operation. Other Aries features include green band analysis for showing coordination and real-time intersection displays that allow the user to assign phases and detectors to images.

Example screens in this section are from Intersection 1 State Street in the U.S. 231 system. Complete data for this system can be found in Appendix B. For each parameter configured, the Aries screen will be displayed with the corresponding controller screen and the Synchro screen (if applicable) from which the data was taken. 


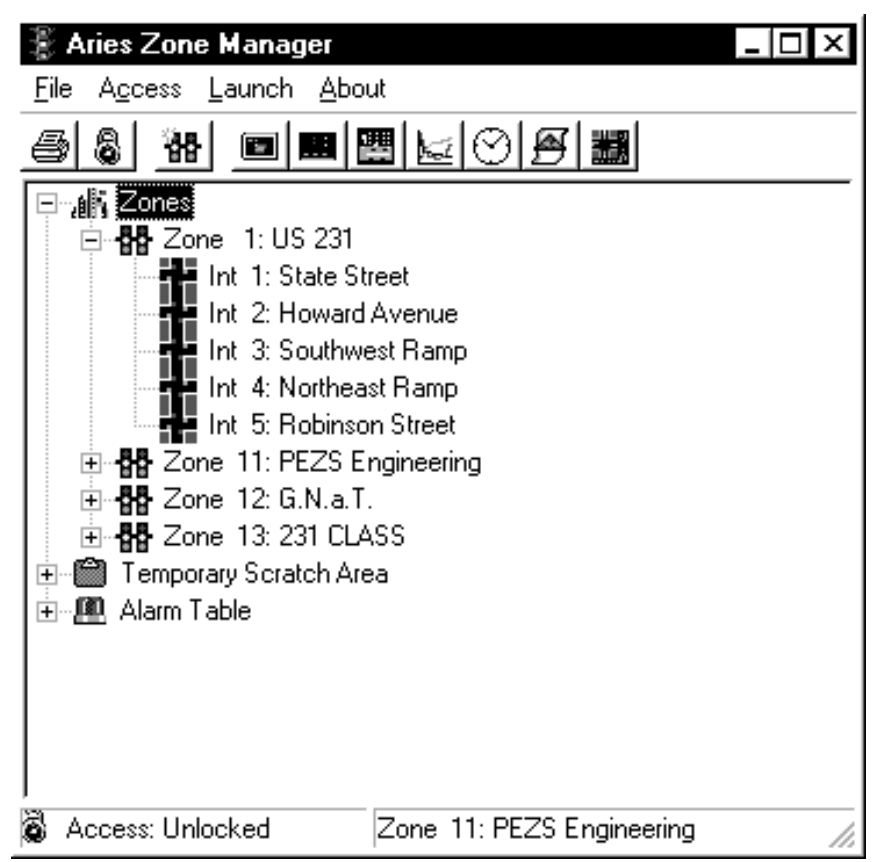

Figure 3-21 Aries Computer Software

\subsubsection{Preparing to Upload}

Aries uploads all data in the controller. However, by design, not all parameters can be configured in Aries to preclude accidentally downloading serious changes to the controller. Certain data can only be entered with the controller keyboard. This data includes that in the configuration submenu, preemption submenu, and phase overlaps. It is extremely important to enter this data before uploading the database to the computer.

\subsubsection{Controller Configuration}

\subsection{Local Procedure:}

Main Menu $\rightarrow$ 1. Configuration $\rightarrow 1$. Controller Sequence

The controller sequence is used to configure the ring structure for the intersection. The ring structure for this intersection is shown in Figure 3-22a. Ring 1 has vehicle phases 1-4 and pedestrian phases 2 and 4 . Ring 2 has vehicle phases $5-8$ and pedestrian phases 6 and 8 . The barriers are located between phases $2 / 6$ and $3 / 7,4 / 8$ and $1 / 5$. In the cabinet, channels $9,10,11$, and 12 correspond to the pedestrian phases. Phase overlaps correspond to the last four load switch in the order A, B, C, and D. The pedestrian and overlap phases are not exclusively timed phases, so they are not included in the ring structure. 


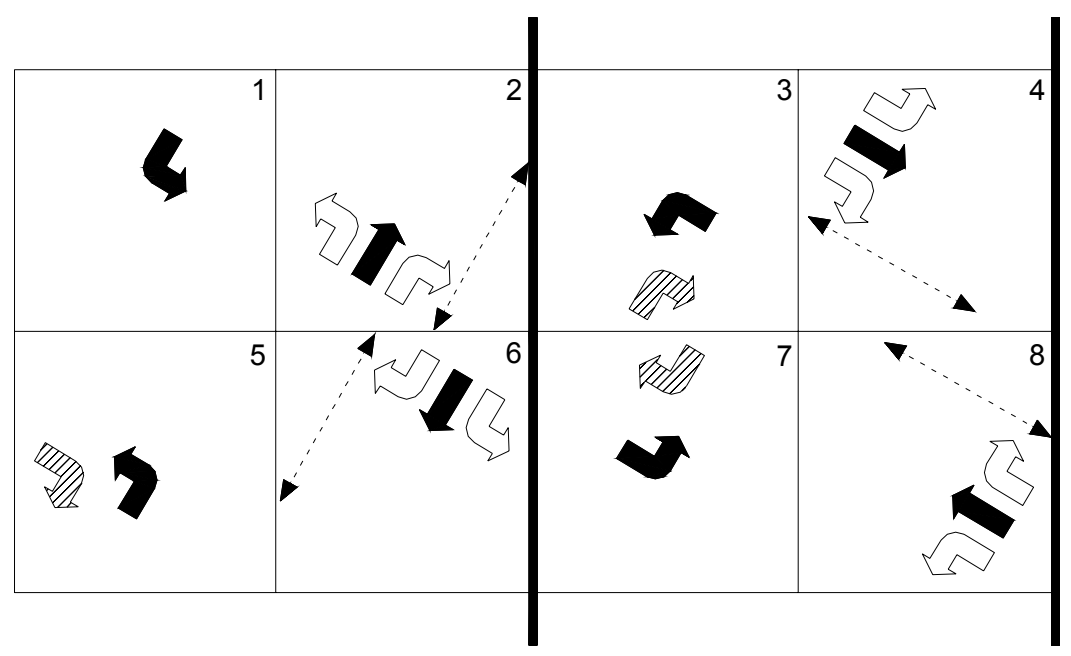

(a) Ring Structure

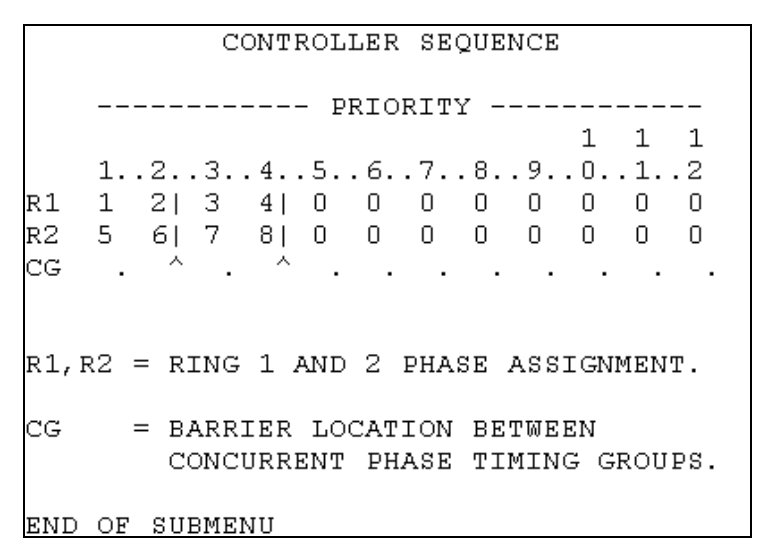

(b) Controller (MM, 1, 1)

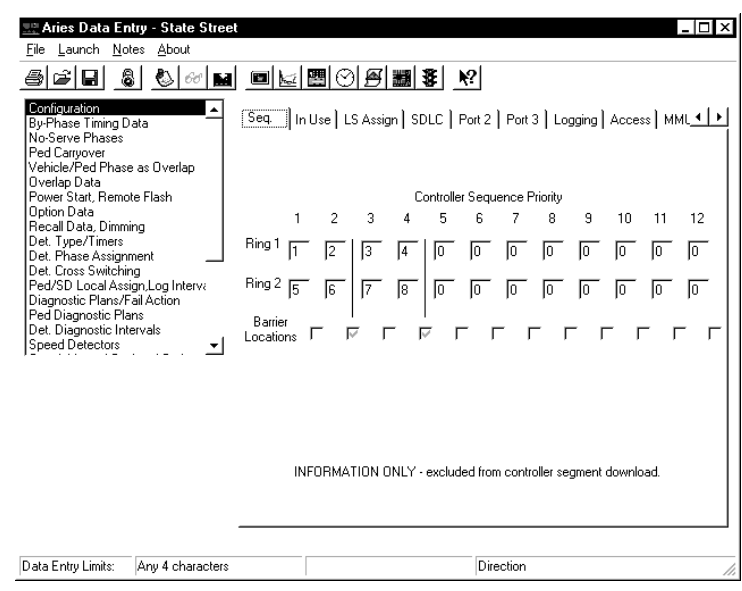

(c) Aries (after upload)

Figure 3-22 Controller Sequence

\section{Main Menu $\rightarrow$ 1. Configuration $\rightarrow 2$. Phases in Use}

All eight vehicle phases are not always used. For example, the intersection could be a Tintersection or not all of the left-turns protected. In these cases, the phases that are not being used can be omitted to prevent any confusion. If the phase is omitted and timings are accidentally entered, Aries will alert the user of this inconsistency.

1. In the Phases in Use screen (Figure 3-23a), place an ' $X$ ' under all of the phases that are in use.

2. Also, if exclusive pedestrian phases are being used, place an ' $X$ ' under the pedestrian phase in the second row (Figure 3-23a). 
3. In the Aries screen (Figure 3-23, Item 3), the phases can be labeled with their direction movement. This data is not present in the controller; it only exists to aid the programmer in the Aries environment.

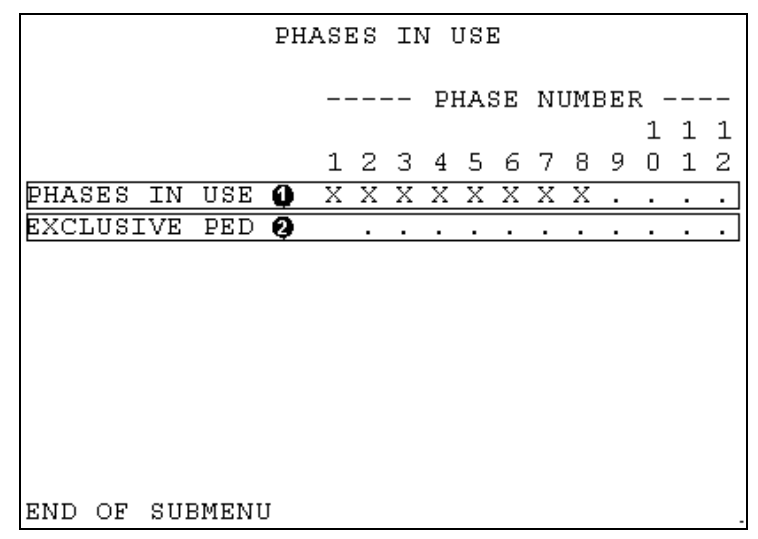

(a) Controller (MM, 1, 2)

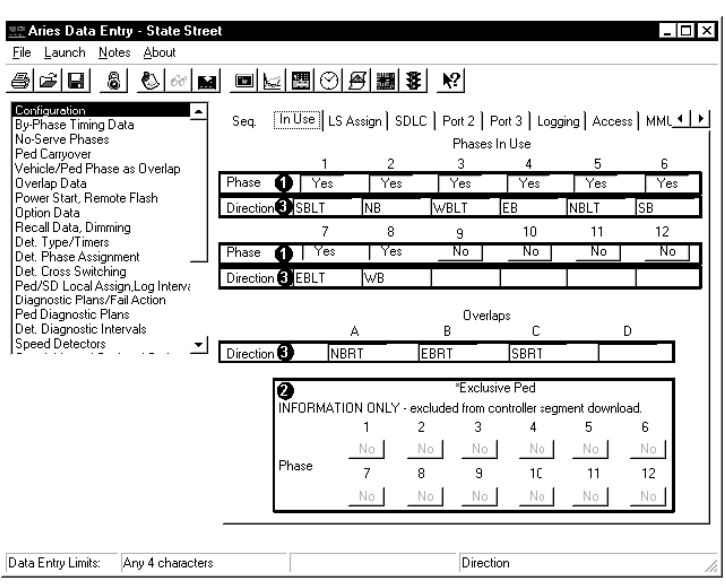

(b) Aries (after upload)

Figure 3-23 Phases in Use

Main Menu $\rightarrow$ 1. Configuration $\rightarrow$ 7. Enable Event Logs

Most agencies log certain controller events, such as detector errors and conflict monitor flashing events as shown in Figure 3-24. INDOT uses alarm 1 to report when the cabinet door has been opened. This data can be transferred using Aries to be viewed or viewed from the controller.

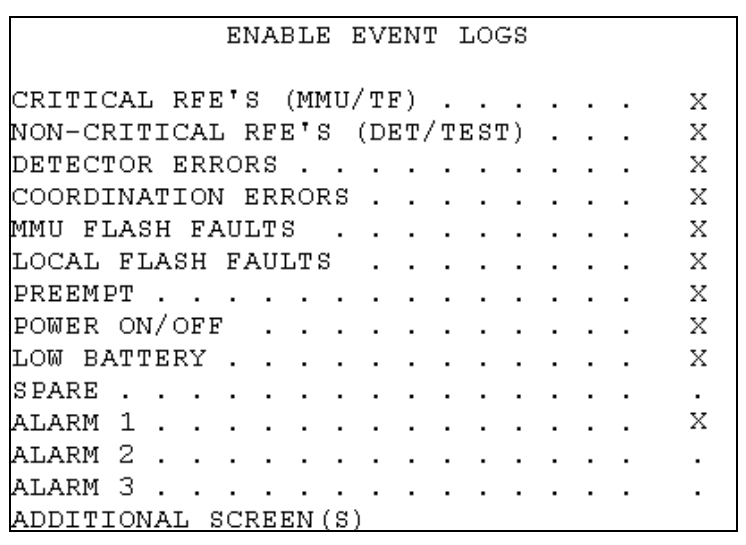

(a) Controller (MM, 1, 7)

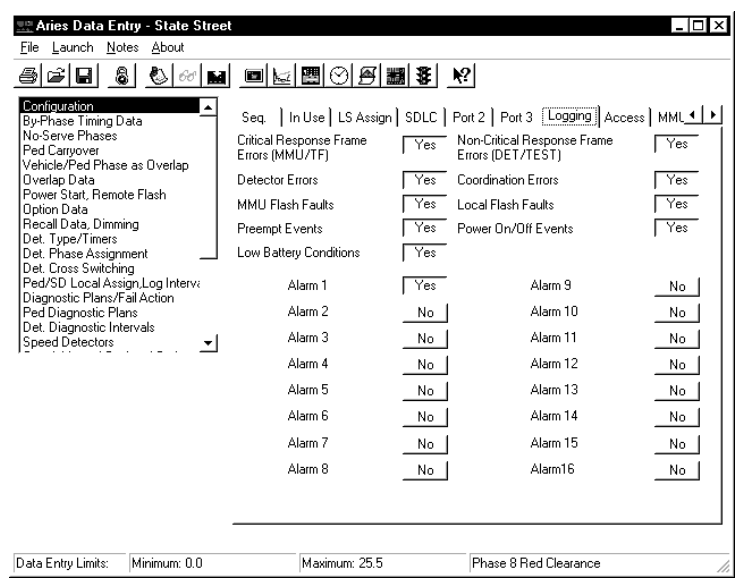

(b) Aries (after upload)

Figure 3-24 Enable Event Logging 


\subsubsection{Overlap Assignment}

\subsection{Local Procedure:}

Main Menu $\rightarrow$ 2. Controller $\rightarrow 5$. Overlap Data

Intersection 1 in this system has right-turn overlaps on three approaches. These overlaps run concurrently with the appropriate left-turn phases as shown in Figure 3-22a. Overlap A, which is the phase 2 northbound right-turn movement, will run concurrently with phase 3 , the westbound left-turn movement. In the screen shown in Figure 3-25a, overlap A should be assigned as a standard overlap with phase 3 . Similarly, overlap B is assigned to phase 5 and overlap $C$ is assigned to phase 7 .

Econolite also provides a "negative green overlap" for right-turn overlap. This allows the rightturn arrow to be displayed during the yellow and all red time of the preceding through movement. This allows the overlap to have additional green time equal to the yellow and red times. 


\begin{tabular}{|c|c|c|c|c|c|c|c|c|c|c|}
\hline & CONTRO & LLER & OVE & RLA & P & DA: & TA & & & \\
\hline & & & & & & & & & & \\
\hline DVERLAP & A. . . . & 12 & 23 & 45 & 6 & 7 & 8 & 9 & 0 & 12 \\
\hline STANDARI & $D \ldots \ldots$ & .. & $x$ & . & $\dot{r}$ & . &. & 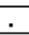 & . & . . \\
\hline PROTECTE & ED $\ldots$ & . . & .. & . . & - & 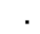 & . & . & . & . . \\
\hline PERMITTE & ED.... & . . & .. & . . & . & . & . & $\cdot$ & . & . . \\
\hline ENABLE I & LAG. . . & . & . & . . & . & . & . & . & . & . \\
\hline ENABLE I & LEAD . . & . . & . . & . . & . & . & . & . & . & \\
\hline SPARE . . & $\ldots \ldots$ & . . & .. & . . & & . & . & & & \\
\hline$A D$ & VANCE GRI & EEN T & IIME & & & & 0.0 & & & \\
\hline LAG & G/LEAD GI & REEN & TIM & IER & & & 0.0 & & & \\
\hline LAC & G/LEAD Y & ELLOW & N TI & MER & & & 0.0 & & & \\
\hline LAG & $G /$ LEAD R & ED TI & LMER & & & & 0.0 & & & \\
\hline DDITION & NAL PAGE & (S) & & & & & & & & \\
\hline
\end{tabular}

(a) Controller Overlap A (MM, 2, 5)

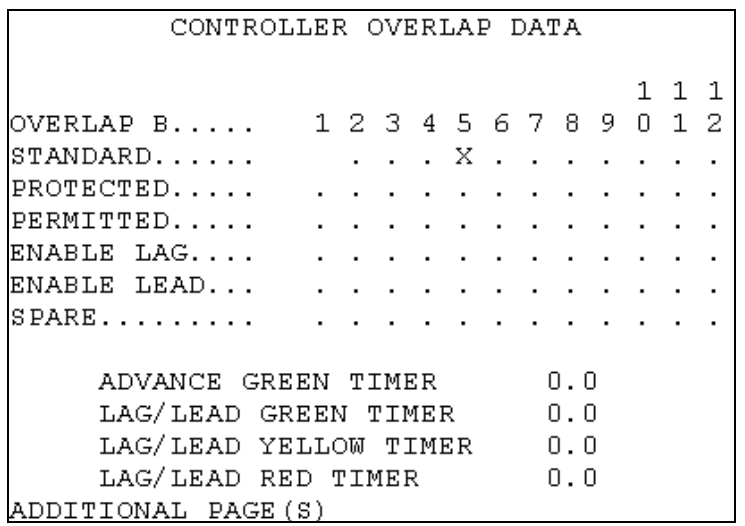

(c) Controller Overlap B (MM, 2, 5)

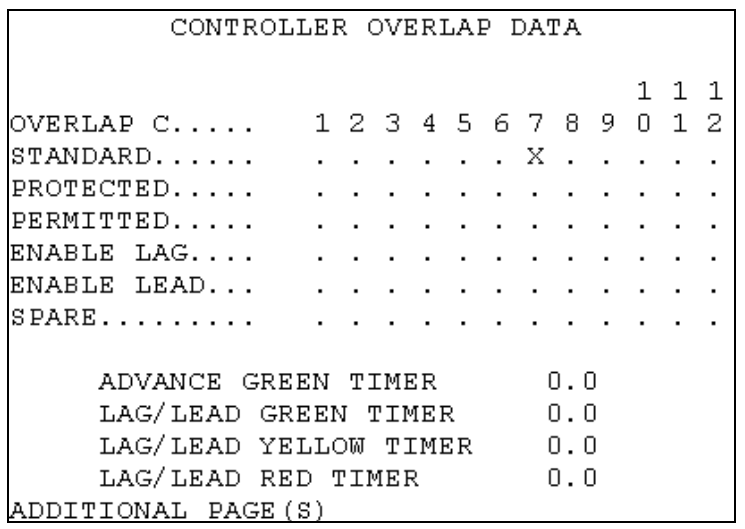

(e) Controller Overlap C (MM, 2, 5)

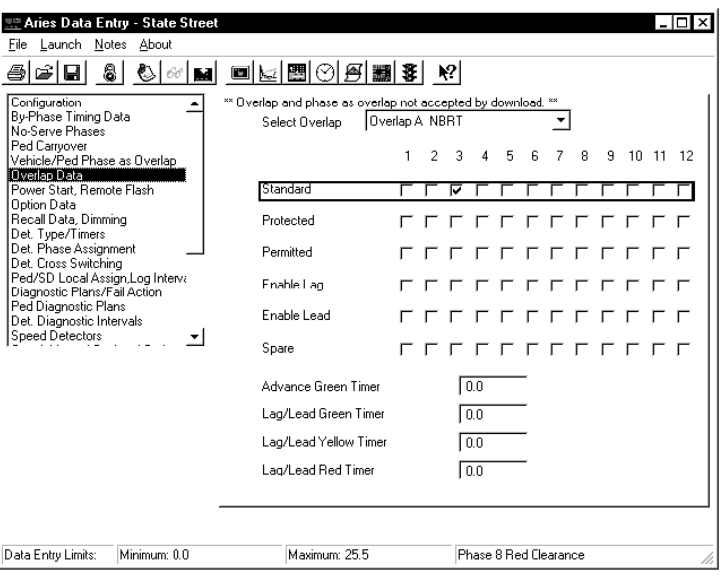

(b) Aries Overlap A (after upload)

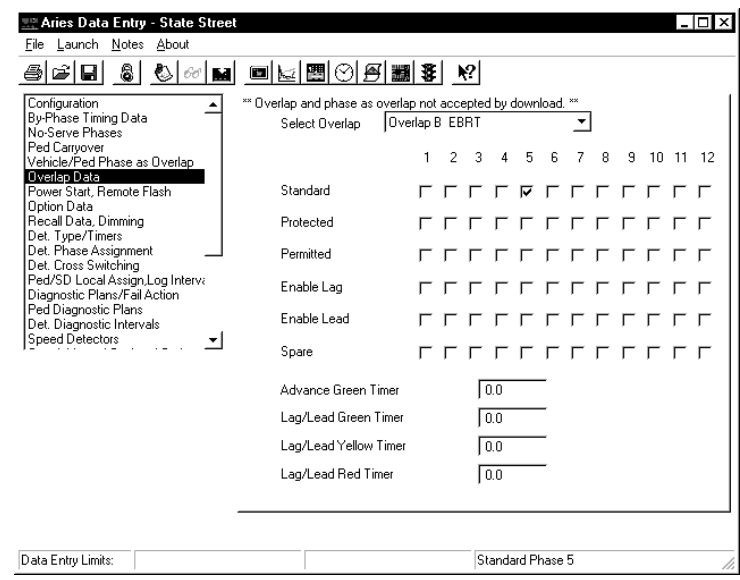

(d) Aries Overlap B (after upload)

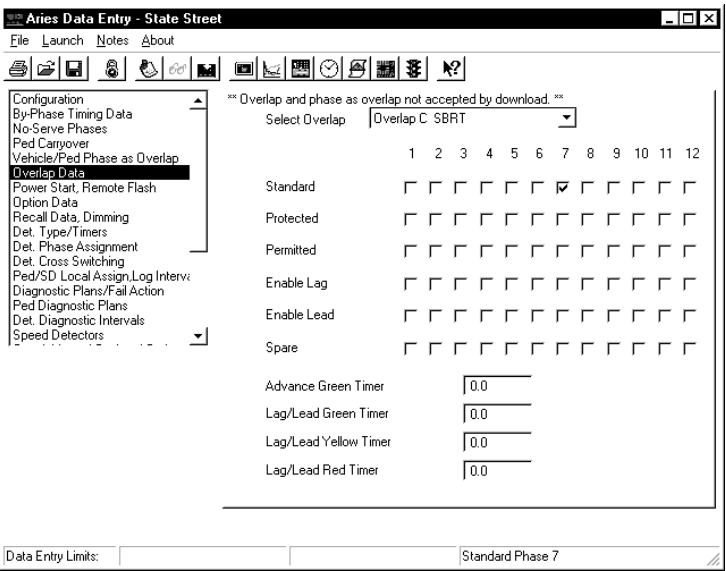

(f) Aries Overlap C (after upload)

Figure 3-25 Phase Overlap Assignments 


\subsubsection{Preemption}

\subsection{Local Procedure}

Main Menu $\rightarrow$ 4. Preemptor $\rightarrow$ 1. Priority Preempt 1

If preemption is used at this intersection, it has to be entered via the keypad and can be configured now or after the database is downloaded back to the controller. There are six priority preemptor and 4 bus preemptor sequences available in the controller. Each sequence can be programmed to serve the desired movements. Sample screens for priority preemptor 1 are shown in Figure 3-26. Since preemption is not commonly used in Indiana, it will not be discussed further. More information on configuration can be found in the Econolite programming manual. ${ }^{19}$

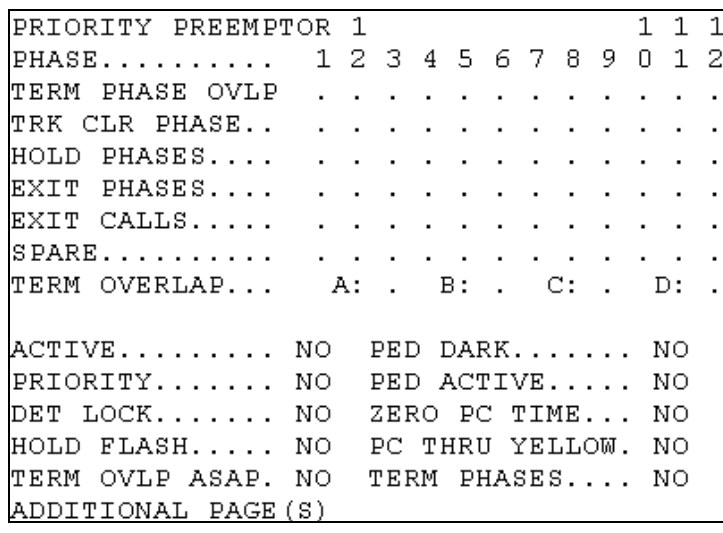

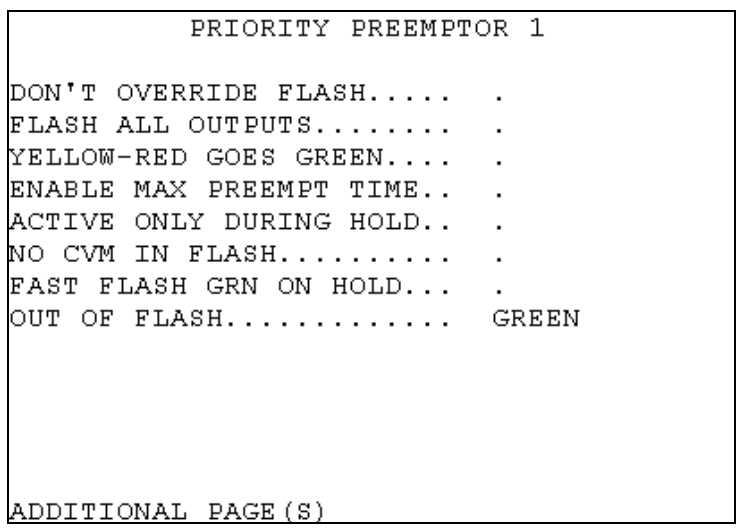

(a) Controller Preemption 1 (MM, 4, 1)

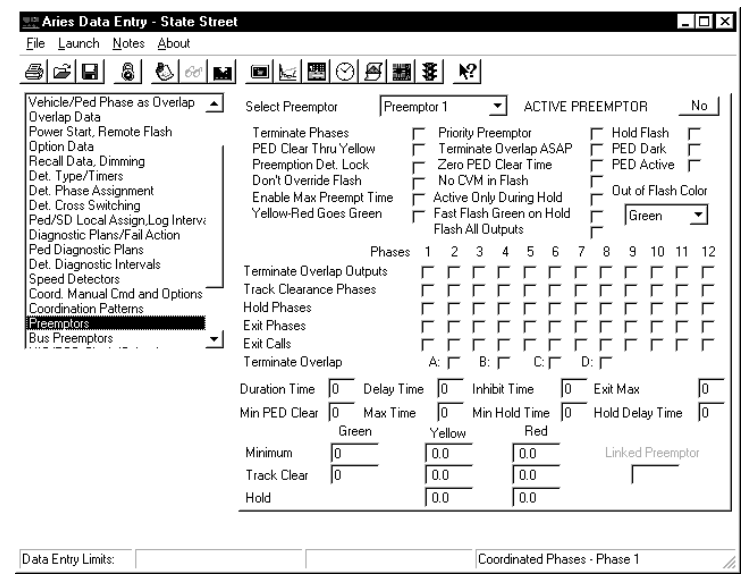

(b) Aries Preemption 1 (after upload)

\section{Figure 3-26 Preemption Configuration}

${ }^{19}$ Econolite Local Controller Programming Manual, Preemption. 


\subsubsection{Setting up an Aries Zone}

\subsubsection{Aries Procedure:}

Right-click on Zones $\rightarrow$ Create a New Zone

A new zone must be created in Aries before data can be uploaded. After Aries has been launched on the computer, find an unused zone number to create a new zone. For this example, Zone 1 will be used. Right click on Zones and create a new zone. Give the zone a unique name to eliminate confusion with other zones. The name should include the main street in the system, such as U.S. 231. The actual phone number to be dialed should be entered here.

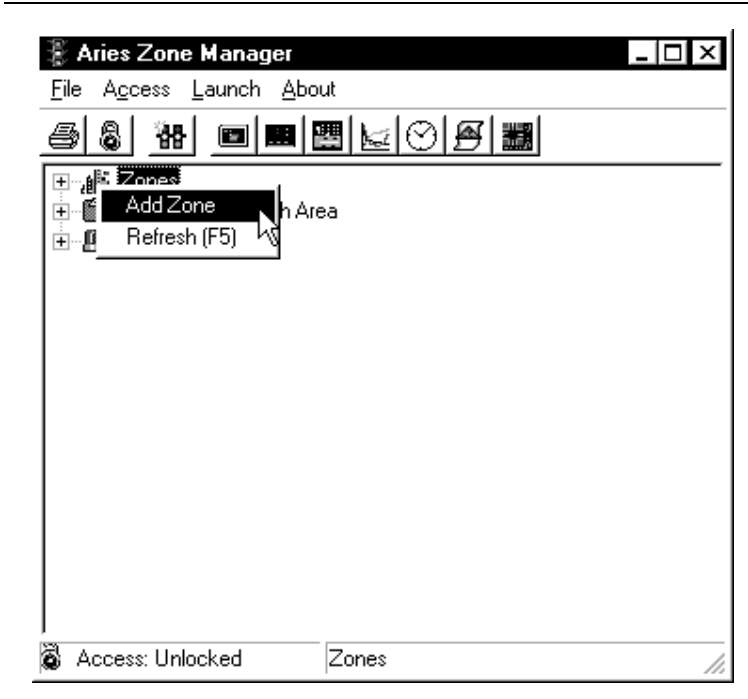

(a) Aries Main Window

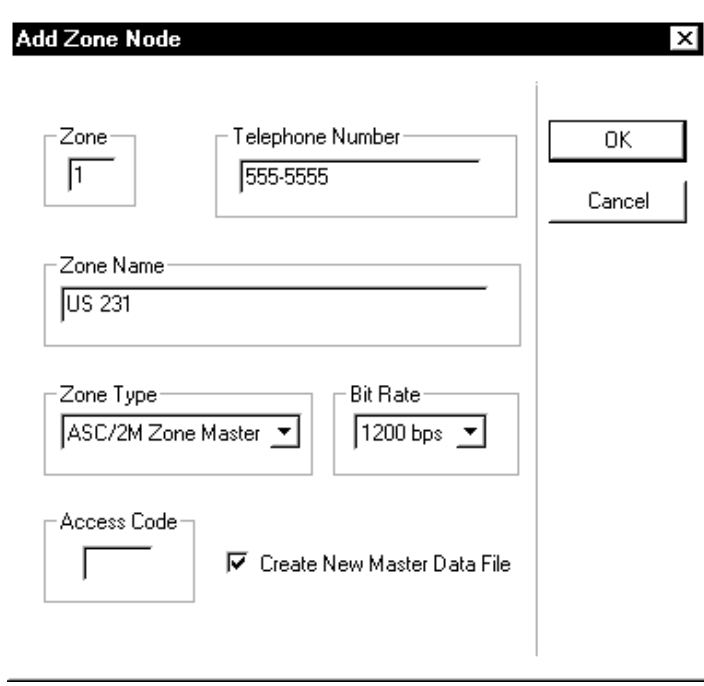

(b) New Zone

Figure 3-27 Aries: Zone Creation

\subsubsection{Master Procedure:}

Main Menu $\rightarrow$ 1. Data Entry $\rightarrow$ 1. System Parameters

Before the computer can communicate with the master, the Aries zone number needs to be entered in the master. In the system parameters, page down to the $5^{\text {th }}$ screen. Enter the master number of the Aries zone that was created. 


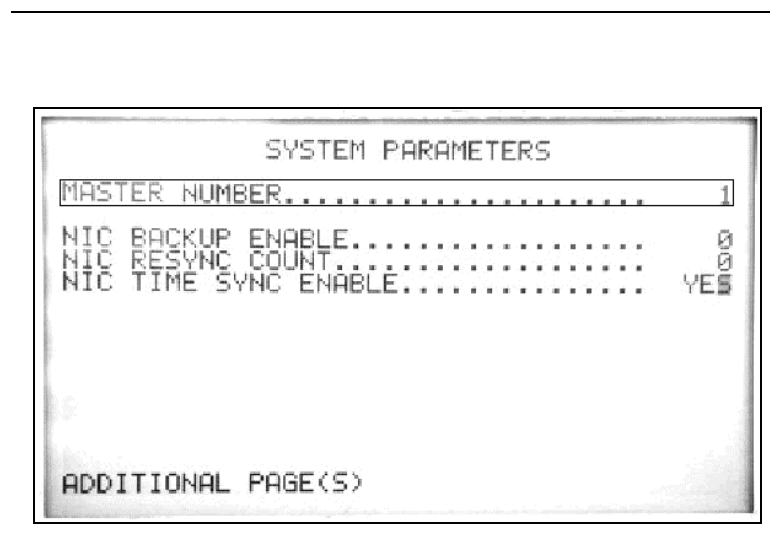

(a) Master Zone Configuration (MM, 1, 1)

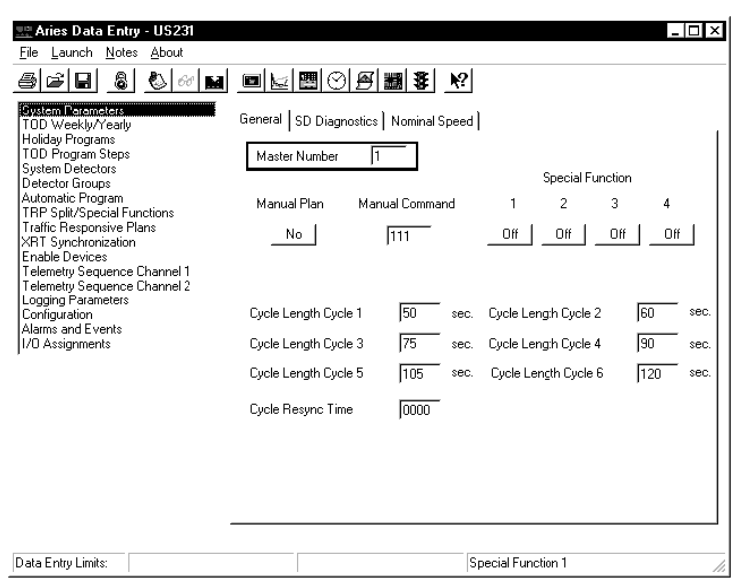

(b) Aries Zone Configuration (after upload)

Figure 3-28 Master Zone Number

Right-click on New Zone $\rightarrow$ Add Intersection

Add each intersection in the system as shown in Figure 3-29a, naming it with the cross street.

The intersection number has to be the same as the telemetry address entered in each controller

in Figure 3-12a.

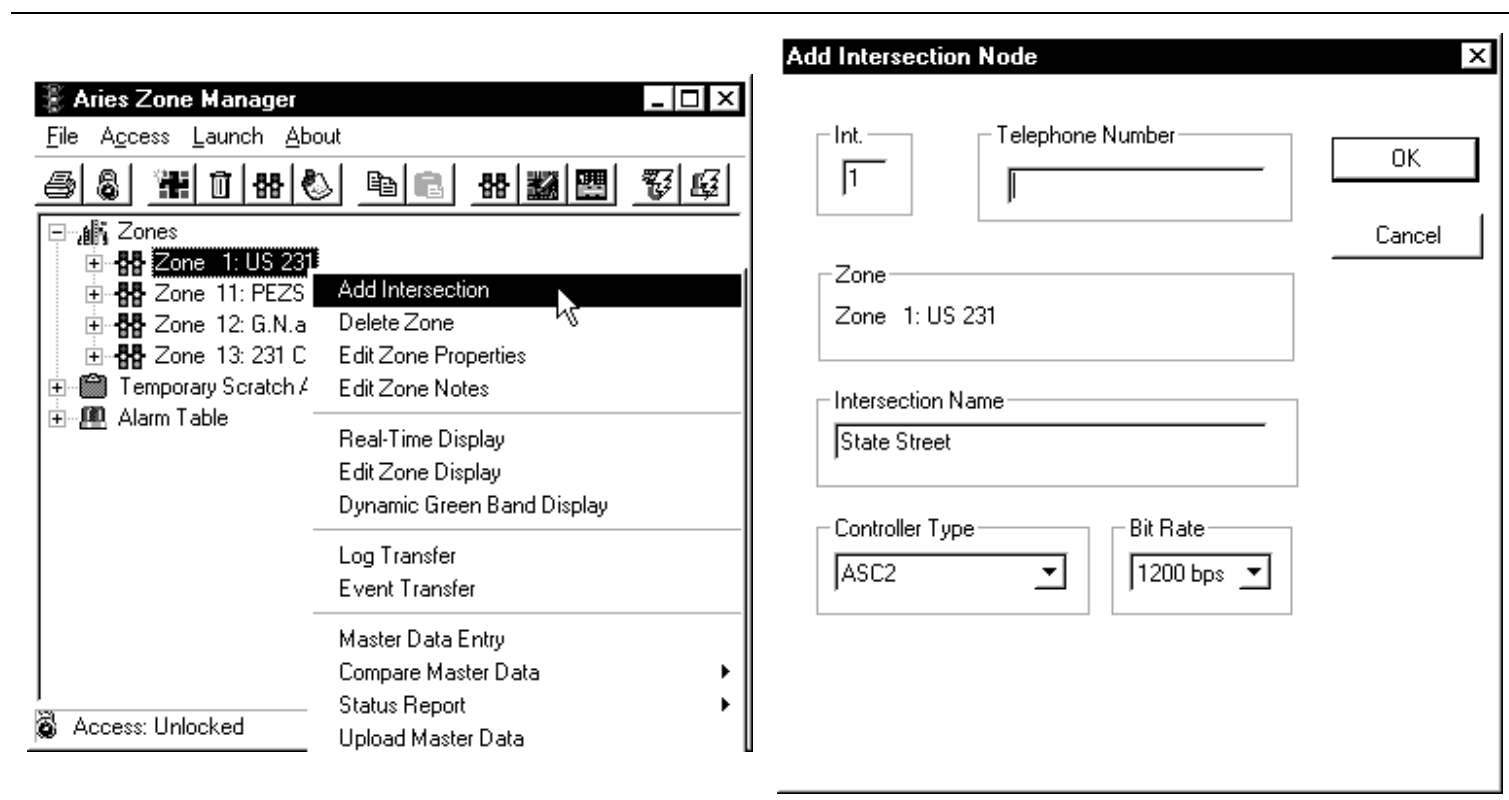

(a) Add Zone Intersection

(b) Intersection Configuration

Figure 3-29 Add Zone Intersections 


\subsubsection{Uploading to Aries}

\subsubsection{Setting Up Communication}

Launch $\rightarrow$ Communications Server $\rightarrow$ File $\rightarrow$ Setup

Before attempting to upload the data, the communications server must be configured for the serial port or modem. The communications server can be launched from the Aries menu. If a channel does not already exist, one must be inserted and edited with the properties button Figure 3-30b. The port number in Figure 3-30c corresponds to the serial port on the computer. The bit rate should be 19,200 for direct connect or 9600 (or less depending on the modem) for a modem. This data should match the data configured in the master in Figure 3-14. In the port options screen in Figure 3-30d, the selection can be made between direct connect or modem. 


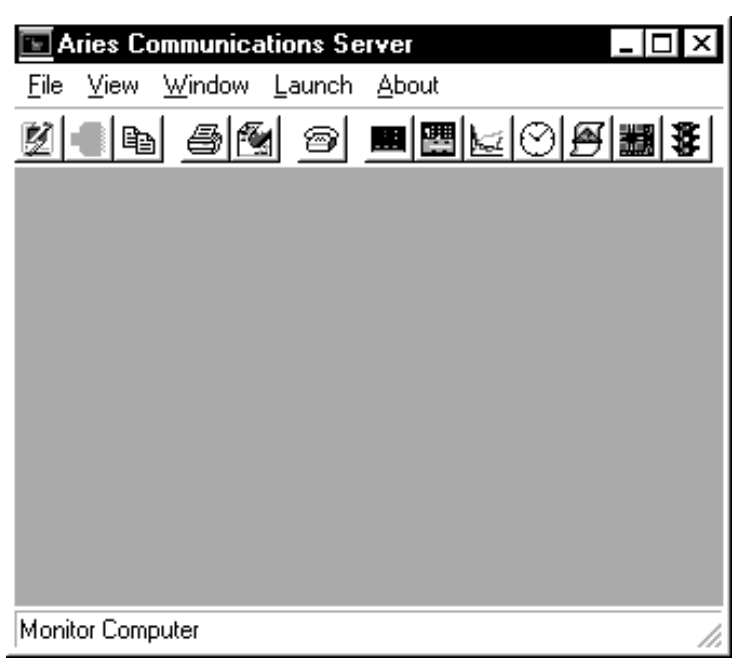

(a) Communication Server

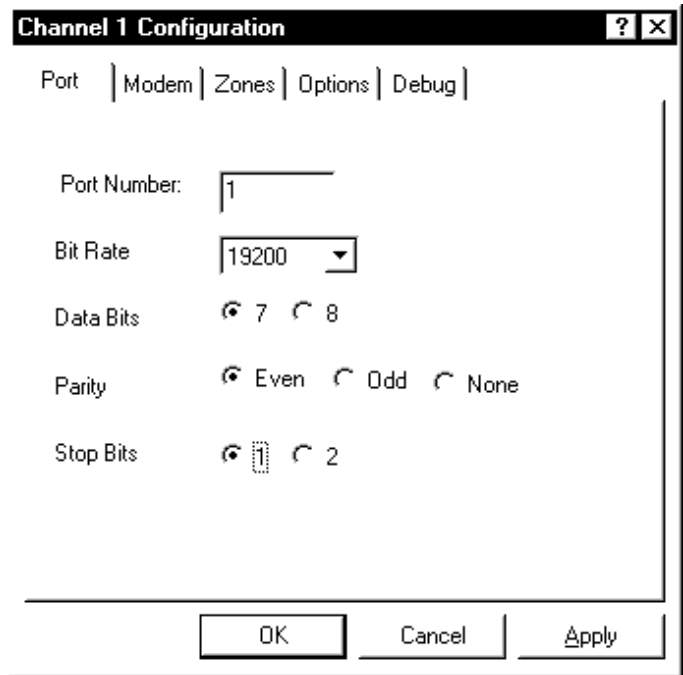

(c) Port Configuration
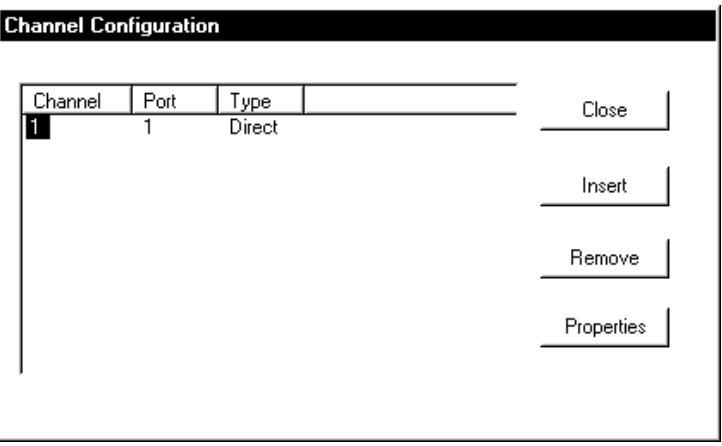

(b) Channel Selection

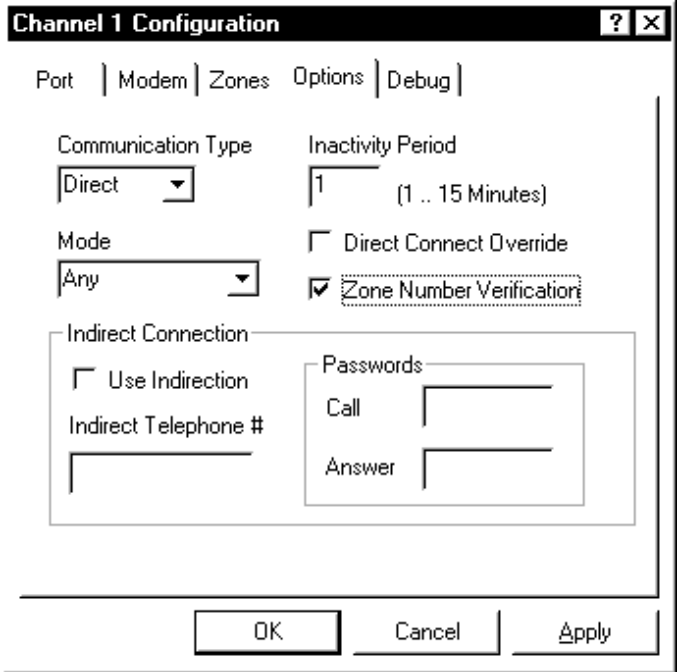

(d) Port Options

Figure 3-30 Aries Communication Server

The quickest way to verify communication is by performing a byte compare for the master controller and a quick compare for each local controller. The master controller should be tested first since local communication is performed through the master. Then each local controller should be tested to make sure that communication is established with all controllers. Figure 3-31a shows the selection of a byte compare for a master. Figure 3-31b shows selection of a quick compare for a local. Figure $3-31 b$ \& c show the communication server screens for successful communication and failed communication. 


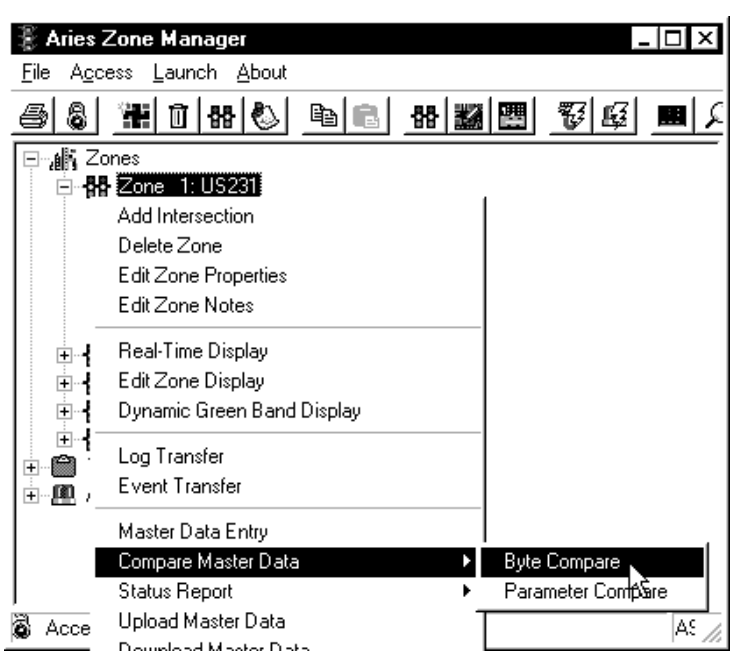

(a) Master Byte Compare

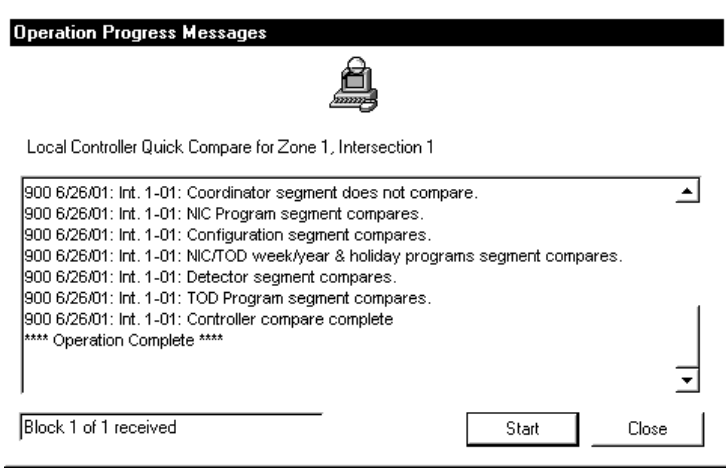

(c) Communication Successful

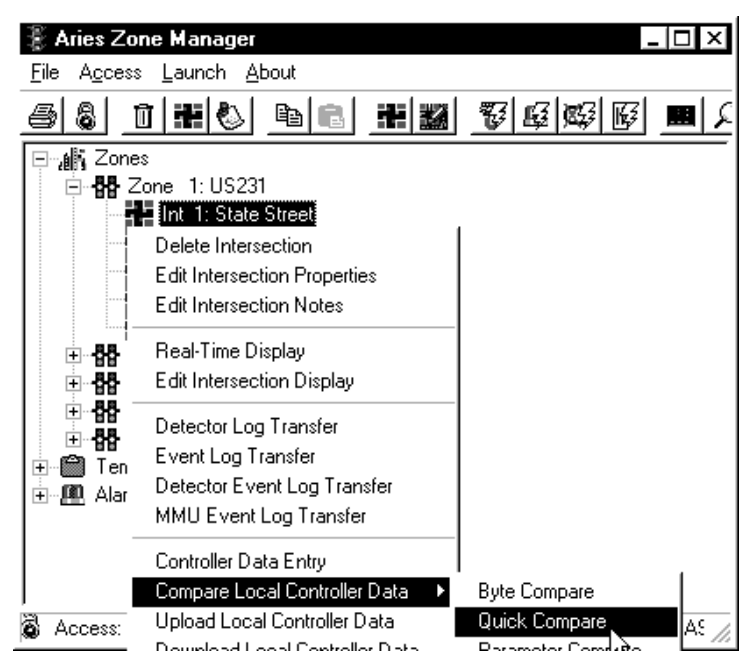

(b) Local Quick Compare

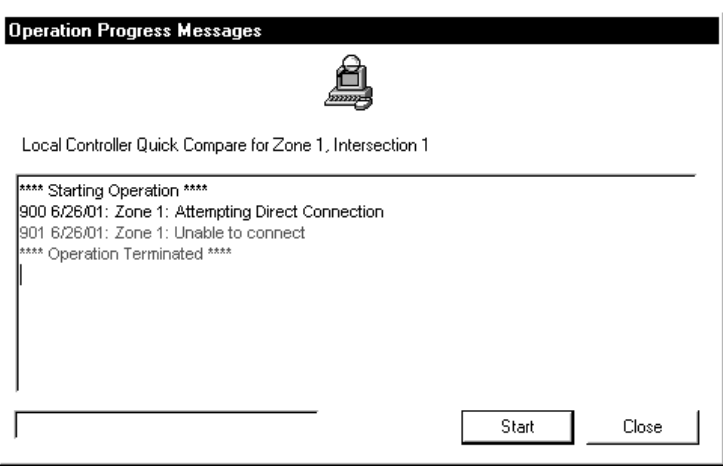

(d) Communication Failed

Figure 3-31 Aries Communication Verification

\subsubsection{Master Upload}

Right-click on New Zone $\rightarrow$ Upload Master Data

Make sure the computer is connected to the master with the proper cable. Aries should be able to upload the master database. After clicking 'Upload Master Data', the Aries communication server will launch. If the communication server launches successfully, the upload can be started. When the data is uploaded, Aries will automatically save the data. If Aries could not connect to the Master, check the cable and the communications server configuration (computer com port, data transfer rate, etc). Do not proceed until communication has been established. 


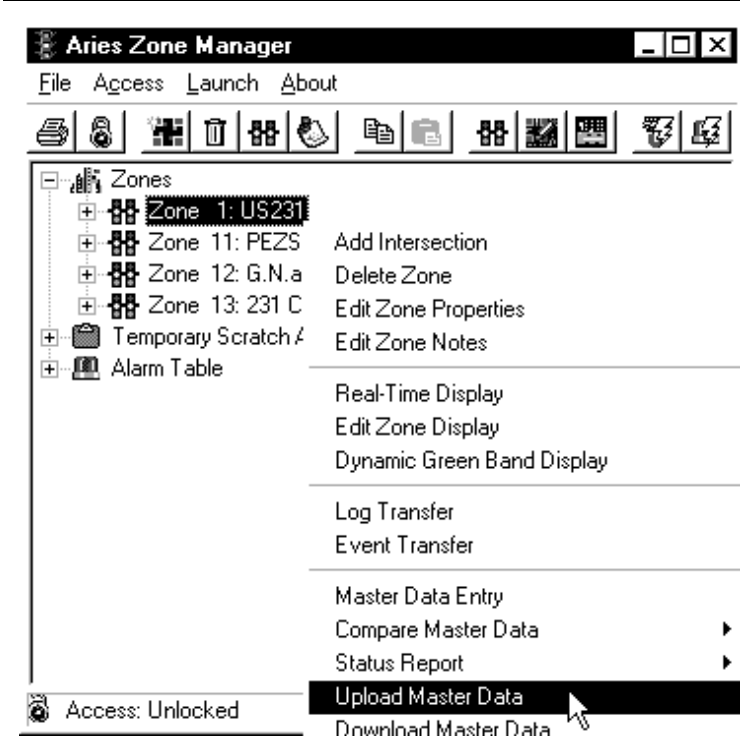

(a) Upload Master Data

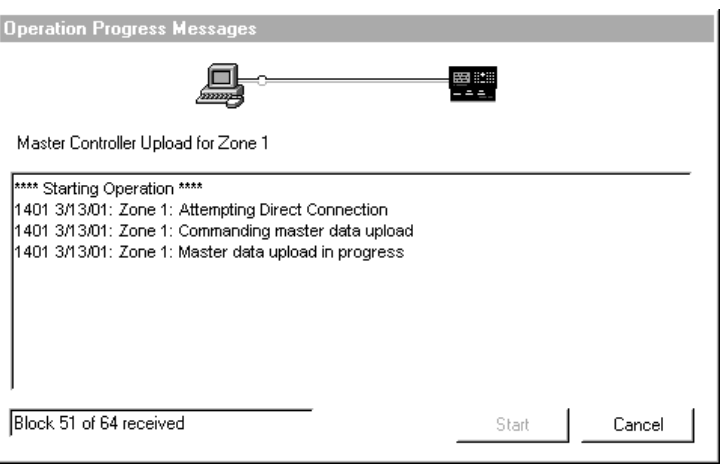

(b) Data Uploading

Figure 3-32 Upload Master Database

\subsubsection{Local Upload}

Right-click on Intersection $\rightarrow$ Upload Intersection Data

Upload each intersection database, by right clicking on that intersection. Save all data after it is uploaded. A convenient tool for uploading all controller databases on a system is the File-BatchUpload Command. This allows an operator to upload all controller information from all controllers in the system without having to repeatedly select intersections and click OK. This can be located in the File menu under batch operations.

If the communication server cannot communicate with a specific controller, check the telemetry address and the data transfer rate. After all intersections are uploaded, the controller data can be configured in Aries. 


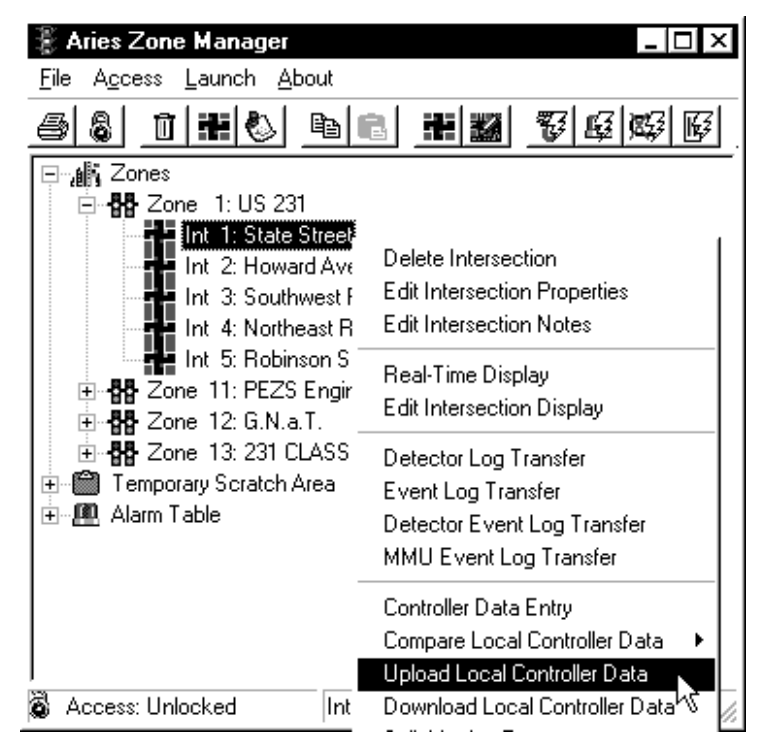

(a) Upload Intersection Data

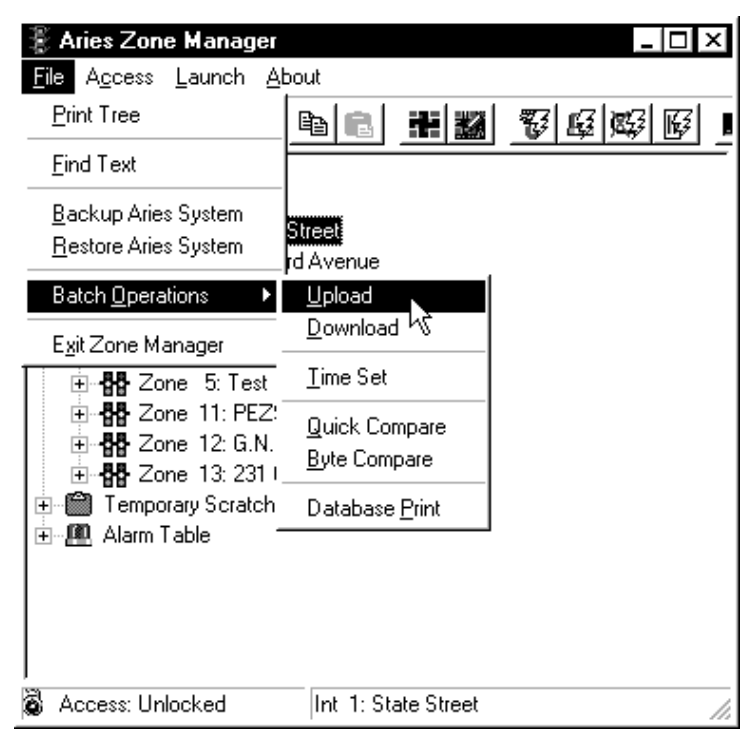

(b) Save Data

Figure 3-33 Upload Intersection Database

\subsubsection{Database Modification}

\subsubsection{Controller Parameters}

\subsection{Timing Data}

The controller timing data in Figure 3-35 includes the minimum data to make the intersection operate. The data in these menus should allow the controller to run the intersection in the event that coordination is lost, but the signals are still active. Parameters that must be entered for the intersection to run include minimum green time, maximum green time, yellow clearance, and red clearance. Be careful entering these parameters directly from a Synchro output. Phase green times and phase splits are not the same. Splits are usually entered in percents and therefore total 100 for each ring. Phase splits include green, yellow, and red times for that particular phase. The data calculated in Chapter 2 (Figure 3-34a) will be used and is shown being entered in Figure 3-35 and Figure 3-36. Note that the table in Figure 3-34a is not in the same sequence as the one shown in Chapter 2. The table in Figure 3-34b outlines the differences in naming conventions for certain parameters between the Econolite controller, Peek controller, and the NEMA TS-2 convention.

Item 1 to be entered into the controller is minimum green. For each phase in the ring structure, the minimum green time should be entered in the corresponding column. Follow the same procedure for items 2-15. 


\begin{tabular}{|c|c|c|c|c|c|c|c|c|c|c|}
\hline & INT & DESCRIPTION (SEC) & 1 & 2 & 3 & 4 & 5 & 6 & 7 & 8 \\
\hline \multirow{15}{*}{$\begin{array}{l}10 \\
2 \\
3 \\
4 \\
5 \\
6 \\
7 \\
6 \\
9 \\
10 \\
11 \\
12 \\
13 \\
4 \\
15\end{array}$} & 1 & MINIMUM GREEN & 5 & 10 & 5 & 7 & 5 & 10 & 5 & 7 \\
\hline & 1 & PEDESTRIAN WALK & -- & 7 & -- & 7 & -- & 7 & -- & 7 \\
\hline & 1 & PEDESTRIAN CLEARANCE & -- & 11 & -- & 11 & -- & 11 & --- & 11 \\
\hline & 1 & VEHICLE EXTENSION & 1.4 & 5.0 & 1.4 & 5.0 & 1.4 & 5.0 & 1.4 & 5.0 \\
\hline & 1 & MAXIMUM EXTENSION & 1.0 & 3.5 & 1.0 & 5.5 & 1.0 & 4.0 & 1.5 & 5.0 \\
\hline & 1 & MAX1 GREEN & 15 & 46 & 15 & 67 & 12 & 48 & 16 & 66 \\
\hline & 1 & MAX2 GREEN & 15 & 46 & 15 & 67 & 12 & 48 & 16 & 66 \\
\hline & 1 & MAX3 GREEN & 17 & 53 & 17 & 78 & 14 & 56 & 19 & 76 \\
\hline & 1 & YELLOW CLEARANCE & 3.2 & 4.0 & 3.2 & 4.0 & 3.2 & 4.0 & 3.2 & 4.0 \\
\hline & 1 & RED CLEARANCE & 2.0 & 1.5 & 2.0 & 1.5 & 2.0 & 1.5 & 2.0 & 1.5 \\
\hline & 1 & SEC/ACTUATION & -- & -- & -- & -- & -- & -- & -- & -- \\
\hline & 1 & MAXIMUM INITIAL & -- & -- & -- & -- & -- & -- & -- & -- \\
\hline & 1 & TIME BEFORE REDUCTION & -- & 15 & -- & 22 & -- & 18 & -- & 25 \\
\hline & 1 & TIME TO REDUCE & -- & 37 & -- & 54 & -- & 45 & -- & 61 \\
\hline & 1 & MINIMUM GAP TIME & -- & 3.0 & -- & 3.0 & -- & 3.0 & -- & 3.0 \\
\hline
\end{tabular}

(a) Controller Parameters from Chapter 2

\begin{tabular}{|c|c|c|}
\hline NEMA TS-2 & Econolite & Peek \\
\hline Minimum Green & Minimum Green & Initial \\
\hline Passage Time & Vehicle Extension & Passage Time \\
\hline
\end{tabular}

(b) Terminology Relations

Figure 3-34 Phase Timings 


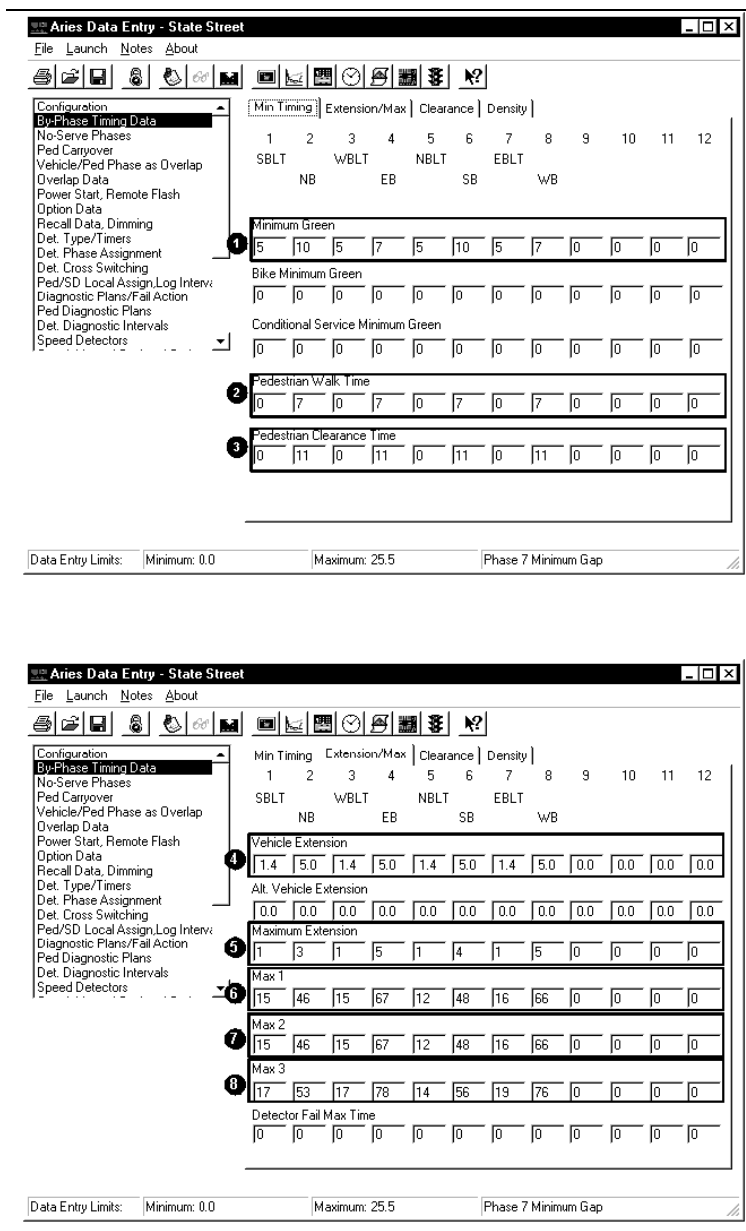

(a) Aries

(b) Controller (MM, 2, 1)

Figure 3-35 Phase Timing (Minimum, Pedestrian, Extension, Maximums) 


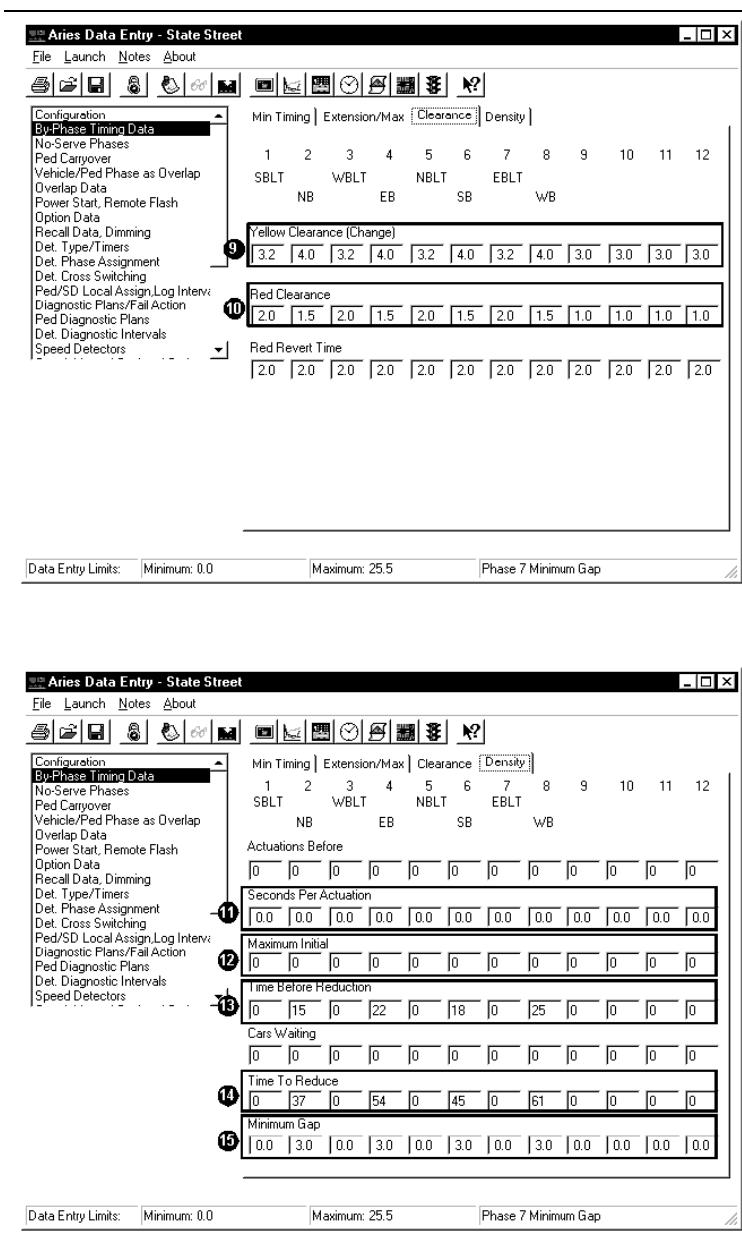

(a) Aries Clearance

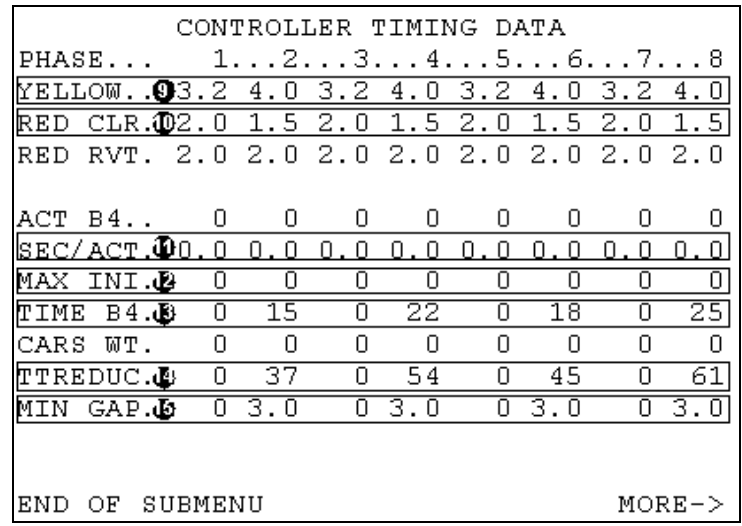

(b) Controller Clearance (MM, 2, 1, PgDn)

Figure 3-36 Phase Timing (Clearance, Density Functions)

\subsection{Recall Data}

Data entered in this menu is in effect when the intersection is running free. Recall data is also entered in the coordination patterns. If a pattern is running, that recall data takes precedence over that shown in Figure 3-37. Consequently, this data should be configured according to how the intersection should run in free mode. Vehicle recall will place a call on a phase while it is red, so that it will be served, but the call does not remain once the phase is served. Recall to max will do the same thing, only once the phase is green, the call remains so that the maximum green time is served. If a movement at the intersection does not have detection, those phases are typically recalled to max.

If all approaches in the intersection have detection, the main street through phases should be placed in soft recall when the intersection is running free. This means that once calls are served 
on the side streets and through movements, the green indication will return to the main street and rest until opposing calls are received. Soft recall would obviously not be needed if the main street phases were in vehicle recall or max recall because the green would immediately be returned due to the recall.

Ped recall will service the pedestrian walk and clearance times during their phases, regardless of the button being pushed. This might be used at an intersection when the pedestrian buttons are not working or are not installed. It is also used in areas with very heavy pedestrian traffic.

At intersection 1 in Figure B-3 in Appendix B, phases 4 and 8 do not have detection, therefore they will be recalled to their max times. Phases 2 and 6 are considered the main street and will be placed in soft recall since they do have detection.

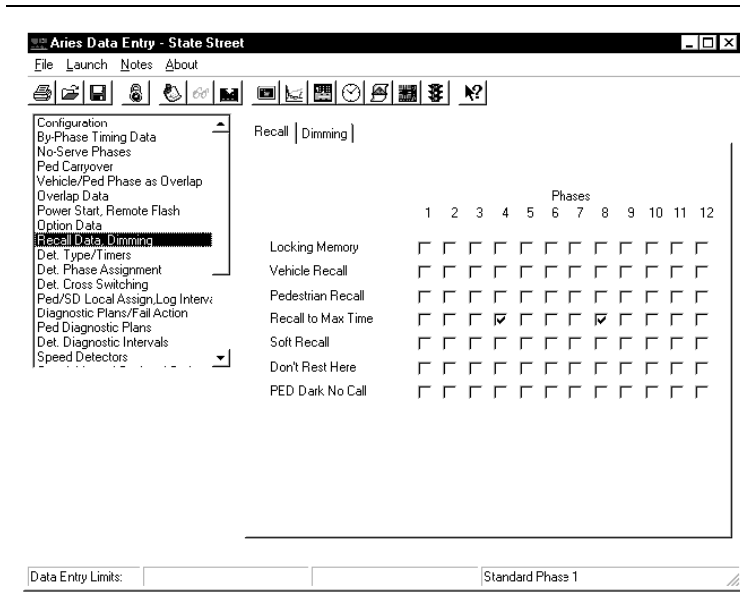

(a) Aries

\begin{tabular}{|c|c|c|c|c|c|c|c|c|c|c|c|c|}
\hline \multicolumn{2}{|c|}{ CONTROLLER } & $\mathrm{R}$ & $\mathrm{EC}$ & A.I & & & $\mathrm{T}$ & & & & & \\
\hline PHASE: & & & & & & & & & & & & \\
\hline PHASE . . . . . & 1 & 2 & 3 & 4 & 5 & 6 & 7 & 8 & 9 & 0 & 1 & 2 \\
\hline LOCKING MEMORY. & . & . & . & . & . & . & 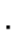 & . & . & . & & . \\
\hline VEHICLE RECALL. & . & . & . & . & . & . & . & . & . & . & & . \\
\hline PED RECALL..... & . & . & . & . & . & . & . & . & . & . & & . \\
\hline RECALL TO MAX.. & . & . & . & $\mathrm{x}$ & . & . & . & $\mathrm{x}$ & . & . & & . \\
\hline SOET RECALL.... & . & . & . & . & . & . & . & - & . & . & & . \\
\hline DON'T REST HERE $^{\prime}$ R & . & . & . & . & $\theta^{\circ}$ & . & . & - & . & . & & . \\
\hline PED DARK N/CALL & . & . & . & . & - & . & . & - & . & . & & . \\
\hline END OF SUBMENU & & & & & & & & & & & & \\
\hline
\end{tabular}

(b) Controller (MM, 2, 4)

Figure 3-37 Recall Data

\subsection{Option Data}

The controller option data parameters that generally need to be configured are dual entry, backup protection, and simultaneous gap. Other option data shown in Figure 3-38 is typically only enabled for special situation in Indiana.

- Dual Entry should be programmed for the through phases on each approach 2, 4, 6, and 8. This will prevent the possibility of having only one phase in green. By programming phase 2 for dual entry, if only phase 5 has a call at an intersection, instead of only phase 5 turning green, phase 2 will also turn green. In the absence of a call in a ring, the dual entry phase in that ring will go green when a phase is green in the same ring. After the 
dual entry is programmed (Figure 3-38a \& b), it must be turned on as shown in Figure $3-38 c$ \& d.

- Backup protection is usually enabled for groups 1 and 2. This prevents reservicing a phase, usually a left-turn, after it has already been active in that cycle. More specifically, if the phase 1 left-turn was served, followed by the phase 6 adjacent through, phase 1 would not be reserviced before phases 3 and 4 were served. Groups 1 and 2 refer to the groups of phases on each side of the barrier in the ring structure. Here, group 1 contains phases 1, 2, 5, and 6. Likewise, group 2 contains phases $3,4,7$, and 8 .

- Simultaneous gap is typically enabled at all intersections in Indiana for safety reasons. This feature controls how the phases function when they gap out. Consider phases 2 and 6 in a standard ring structure that must both terminate in order to cross the barrier. If the phase 2 vehicle extension timer expires and phase 6 is still active, with simultaneous gap enabled, the phase 2 vehicle extension timer can be reactivated. Without it enabled, phase 2 cannot be extended again and will terminate when phase 6 gaps out. However, when intersections are heavily saturated, more effective operation can be achieved if this feature is not enabled. 


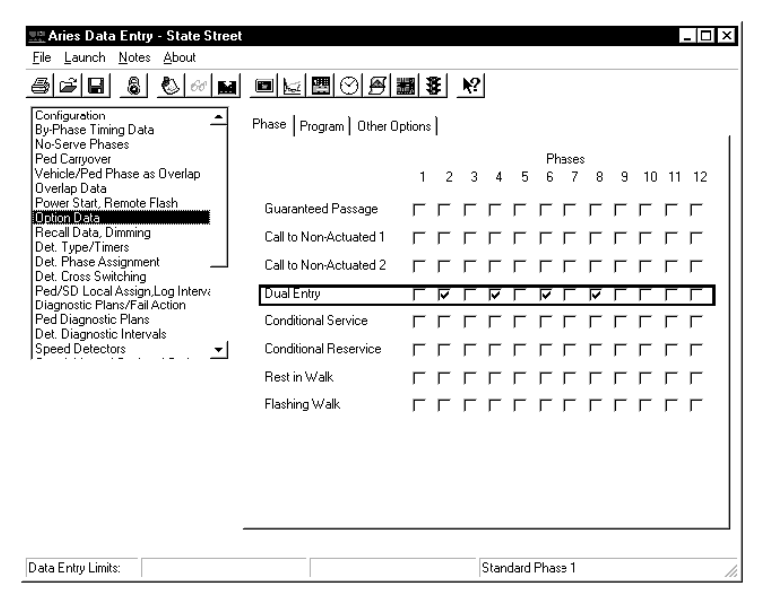

(a) Aries Screen 1

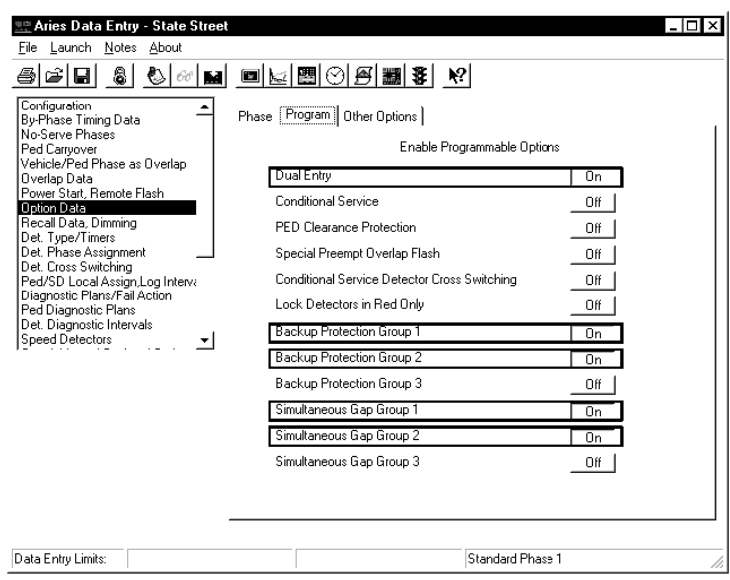

(c) Aries Screen 2

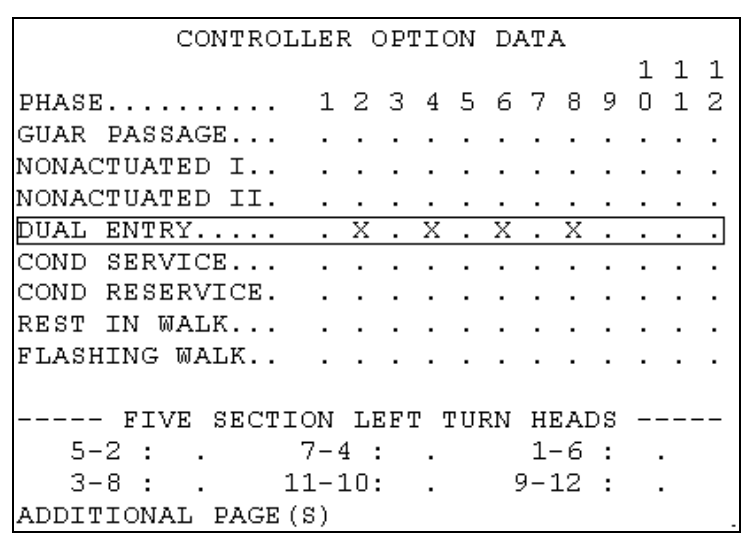

(b) Controller Screen 1 (MM, 2, 9)

\begin{tabular}{|c|}
\hline CONTROLLER OPTION DATA \\
\hline DUAL ENTRY $\ldots \ldots \ldots \ldots \ldots \ldots$ \\
\hline COND SERVICE ENABLE.......... OFF \\
\hline COND SERVICE DET X SWITCHING. . . OFE \\
\hline PED CLR RROTECT............. OFE \\
\hline SPEC PREEMPT OVLP FLASH. . . . . OFE \\
\hline LOCK DETECTORS IN RED ONLY..... OFE \\
\hline RESERVED ................ OFE \\
\hline 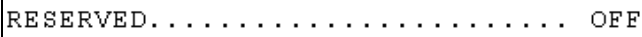 \\
\hline BACKUP PROTECTION GROUP $1 \ldots \ldots$ \\
\hline BACKUP PROTECTION GROUP $2 \ldots$ \\
\hline BACKUP PROTECTION GROUP $3 \ldots \ldots$ OFE \\
\hline SIMULT ANEOUS GAP GROUP $1 \ldots \ldots$ \\
\hline SIMULTANEOUS GAP GROUP $2 \ldots \ldots$ \\
\hline $\begin{array}{l}\text { SIMULTANEOUS GAP GROUP } \\
\text { END OE SUBMENU }\end{array}$ \\
\hline
\end{tabular}

(d) Controller Screen 2 (MM, 2, 9, PgDn)

Figure 3-38 Controller Option Data

\subsubsection{Coordination}

Coordination plans in Indiana are typically generated by the Synchro design software. Synchro provides cycle lengths, splits, and offsets for given volumes. As was discussed in Chapter 2, a pattern should be generated for the six time periods shown in Table 2-5.

\subsection{Local Procedure}

The Synchro outputs for three different coordination plans are shown below with the corresponding Aries screen and Controller screen. Before these parameters are configured, the following coordination options should be entered.

1. Split units can be entered in seconds or percentages. Splits in percentages are more useful because if the cycle length is changed, the splits do not have to be recalculated. 
2. Offsets units can also be entered in seconds or percentages, but usually make more sense to the programmer in seconds.

3. Interconnect format should be standard.

4. Interconnect source should be telemetry. If the system is not interconnected, the source should be Non-interconnected (NIC).

5. Transition refers to the type of algorithm the controller uses to get back into coordination if it gets out of coordination. The choices are smooth, add only, and dwell. Add only adds time to get back into coordination, where smooth can add or subtract. Dwell is the quickest way to get back into coordination, but it can lead to increased delay at the intersection because phases are extended, and is rarely used. Smooth and add only are the most common transition procedures used.

6. Actuated Coordinated Phases are enabled at this intersection because the coordinated phases have detection. This does not always apply and doesn't apply to any other intersection in this system. Standard practice is to not have actuated coordinated phases. These phases would simply be placed in maximum recall.

7. Inhibit Max is enabled because the maximum times should be inhibited so that the phase will be terminated due to split times, rather than the maximum time. In all three coordination patterns, phases 4 and 8 recall to their maximum times because they have no detection at intersection 1. This is not a typical scenario. Usually, phases 2 and 6 have no detection and are recalled to their max times. Since max times are inhibited, the full split time will be served for phases 4 and 8 . If these phases were not recalled to their max times, they would never be served because they could never be called.

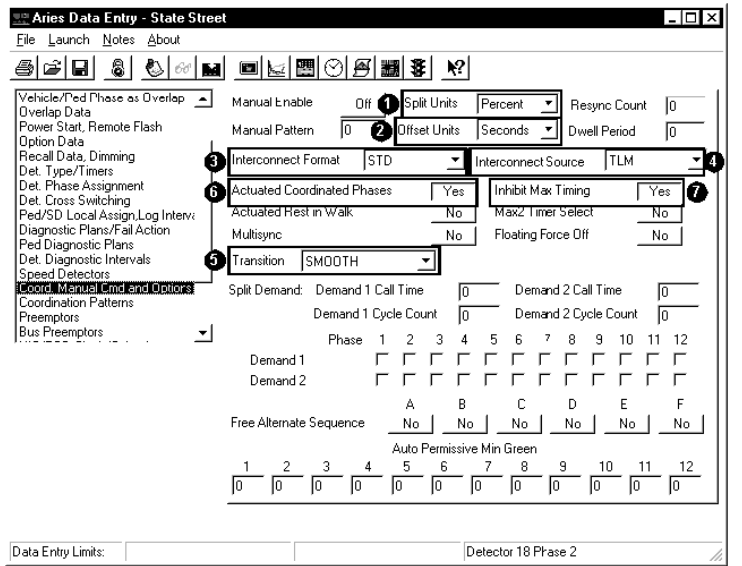

(a) Aries

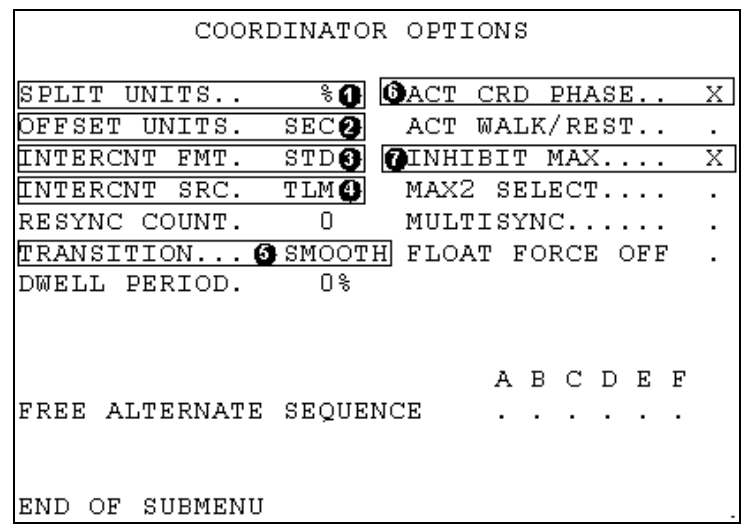

(b) Controller (MM, 3, 1)

Figure 3-39 Coordination Options 
Up to 64 coordination patterns can be programmed in the local controller. Each pattern can have a different cycle, phase splits, and intersection offset. Typically, various coordination patterns would be developed based on different volumes. These different coordination patterns can be selected during a certain time of the day, day of the week, or day of the year. The selection of these coordination patterns will be discussed later.

8. Coordination Pattern number can be selected from 1 to 64 . This number is what is referenced in a NIC program step.

9. Cycle Length can vary from 30 to 255 seconds. Typically, shorter cycle lengths are used during low-volume scenarios and longer cycle lengths are used during high-volume scenarios.

10. $\underline{\mathrm{C} / \mathrm{O} / \mathrm{S}}$ (Cycle/Offset/Split) is another way of referring to the coordination plan. If the same cycle length is used for a different coordination plan, but the splits and offset are changed, the two plans might be $1 / 1 / 1$ and $1 / 2 / 2$. Since the three plans used here all have different cycles, offsets, and splits, they are $1 / 1 / 1,2 / 2 / 2$, and $3 / 3 / 3$.

11. Offset value is entered here in seconds. In Synchro, the offset can be referenced from various points in the ring structure. In coordinated systems, the only predictable point from which to reference an offset is the end of main street green. However, the default offset reference in Econolite controllers is the beginning of main street green. The reference used here will be beginning of main street green.

12. Splits are entered here in percentages. Phases $1-4$ have to total $100 \%$ and phases $5-8$ have to total $100 \%$. Disregard error messages saying that the splits do not total $100 \%$ until you enter phases 4 and 8 . After these are entered, there should be no error message. If the minimum green time error shows up, go back and check your min green times as discussed in Section 3.4.4.1.1. Please note that the phase percentages in the Synchro output do not always add up to $100 \%$. The phase splits entered into the controller have to be adjusted.

13. Coordinated Phases are usually the main street through phases. In this example they are phases 2 and 6 .

14. Vehicle Max Recall is enabled on all phases that have no detection. Here, that is phases 4 and 8 . Typically, it would be the coordinated phases 2 and 6 . 


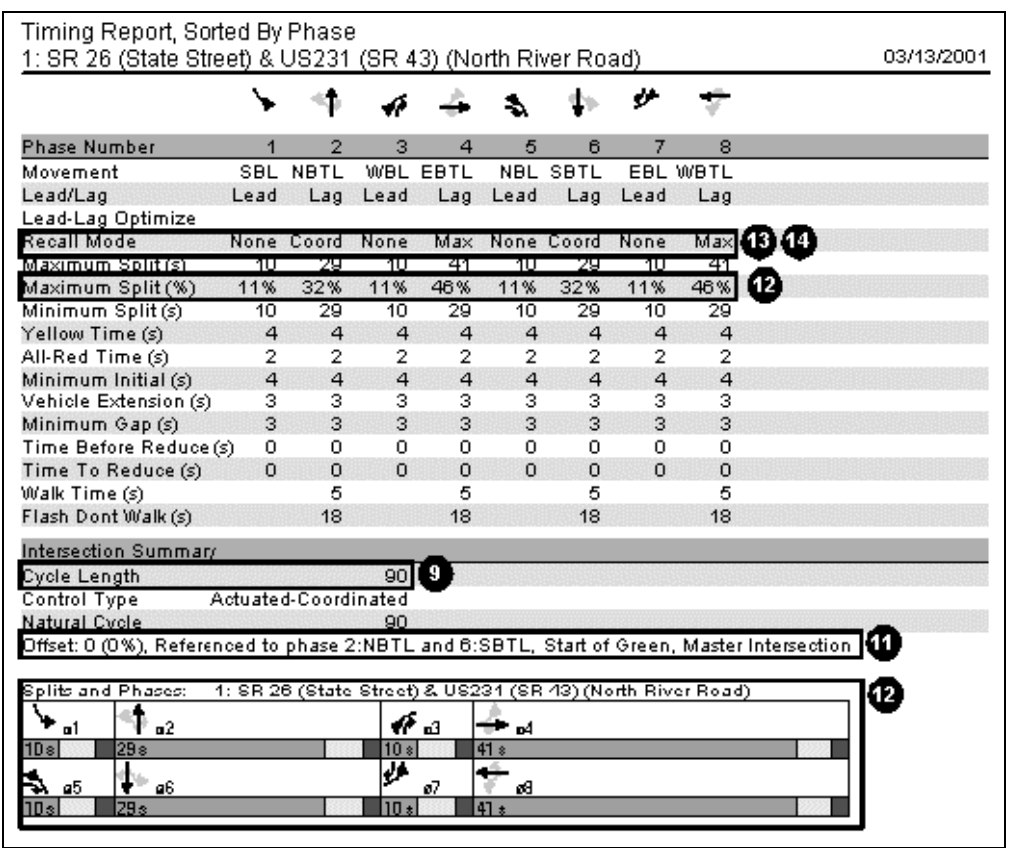

(a) Synchro

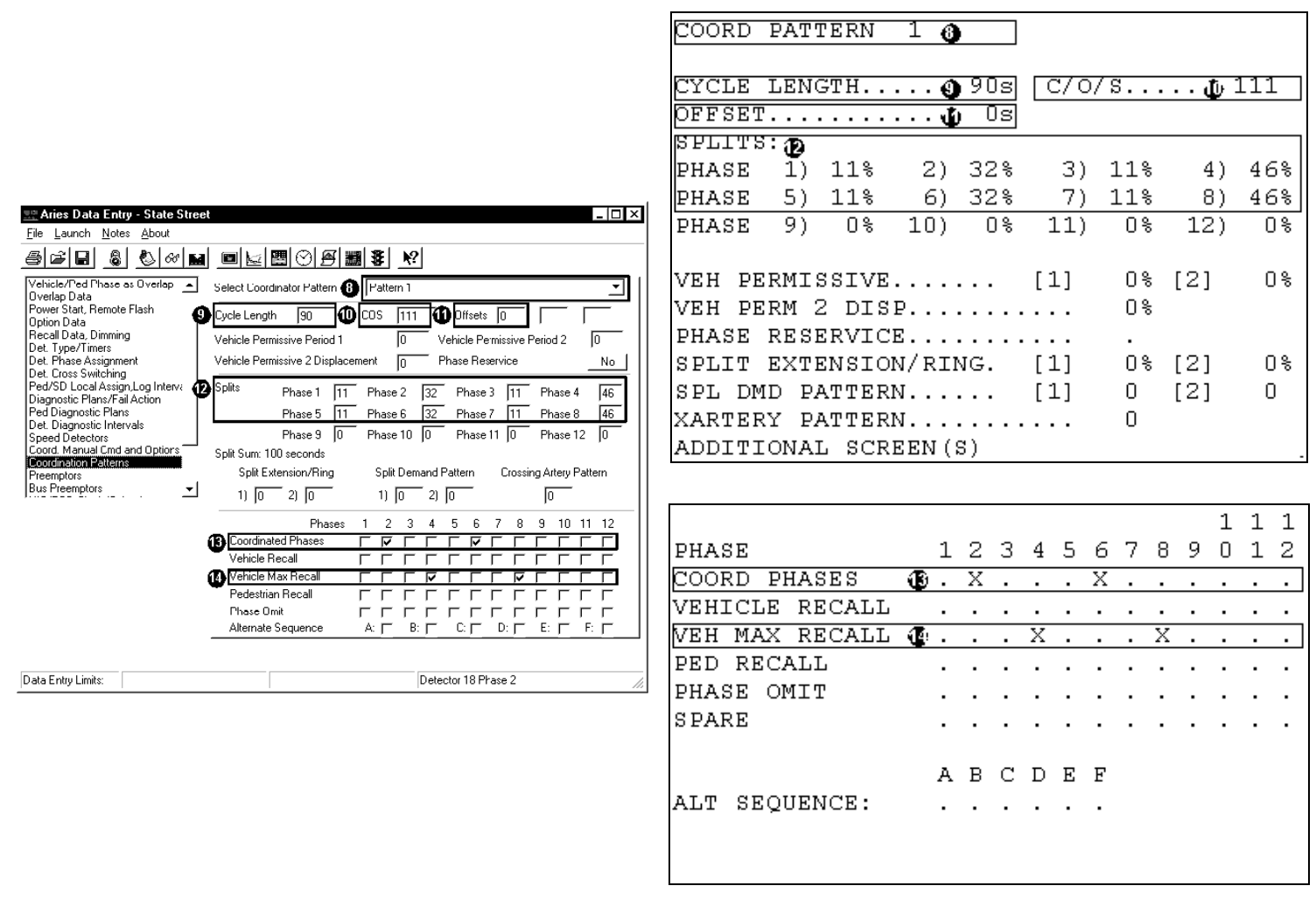

(b) Aries

(c) Controller (MM, 3, 4)

Figure 3-40 Coordination Pattern 1 


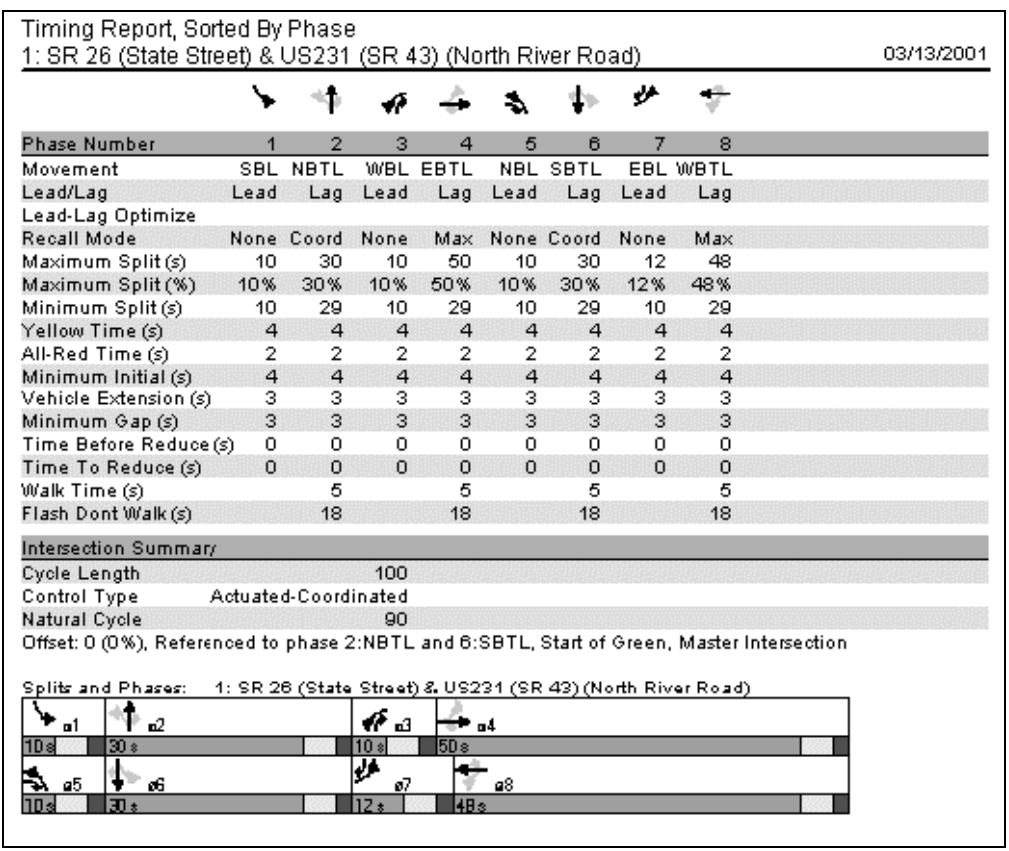

(a) Synchro

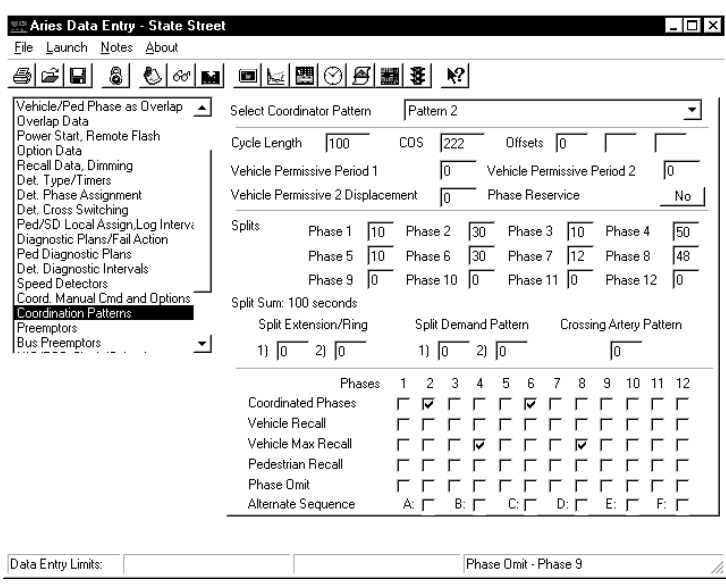

(b) Aries

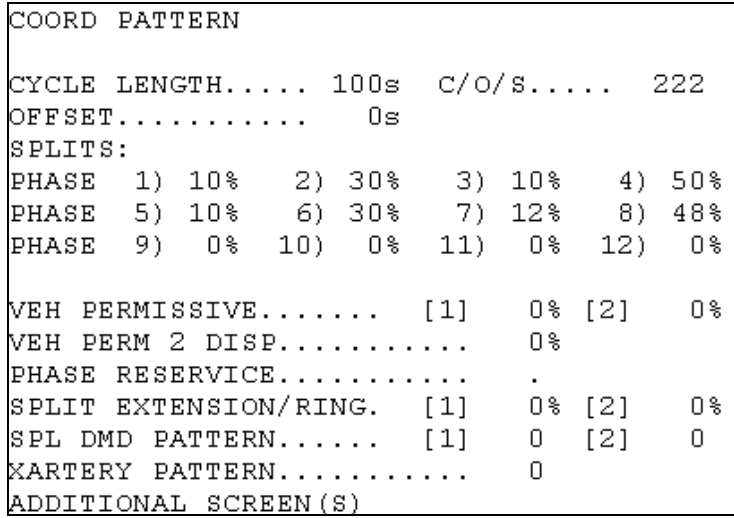

\begin{tabular}{|c|c|c|c|c|c|c|c|c|c|c|c|}
\hline PHASE & 1 & 2 & 3 & 4 & 5 & 6 & 7 & 8 & 9 & ] & $\begin{array}{l}1 \\
1\end{array}$ \\
\hline COORD PHÄSES & . & $\mathrm{X}$ &. & . & . & $\mathrm{x}$ & " & . & . & & . \\
\hline VEHICLE RECALI & - & - & . & . & . & . & . & . & • & & . \\
\hline VEH MAX RECALL & . & . & . & $x$ & . & . & . & $\mathrm{X}$ & . & & . \\
\hline PED RECALL & - & . & . & . & . & . & . & . & • & & . \\
\hline PHASE OUIT & . & . & . & . & . & . & . & . & . & & . \\
\hline SPARE & . & . & . & . & . & . & . & . & . & & \\
\hline & & & $\mathrm{C}$ & $\mathrm{D}$ & E & $\mathrm{E}$ & & & & & \\
\hline MLT SEQUENCE : & . & & . & . & . & . & & & & & \\
\hline
\end{tabular}

(c) Controller (MM, 3, 4, PgDn(2))

Figure 3-41 Coordination Pattern 2 


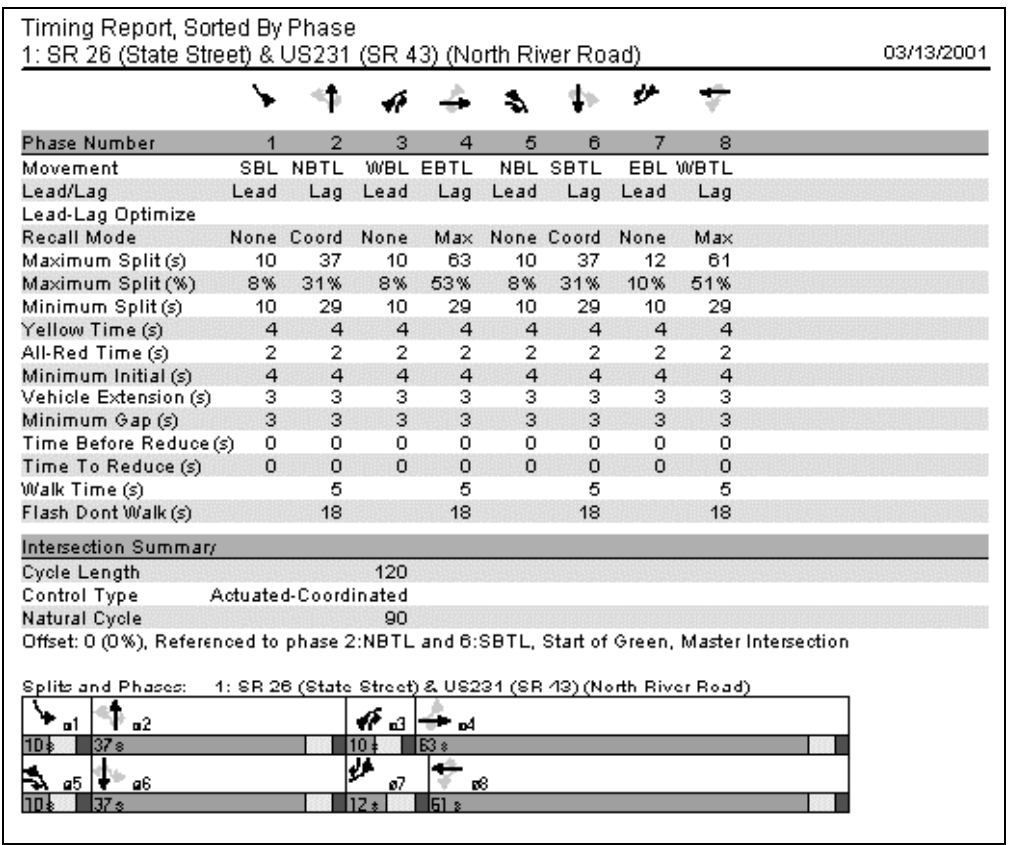

(a) Synchro

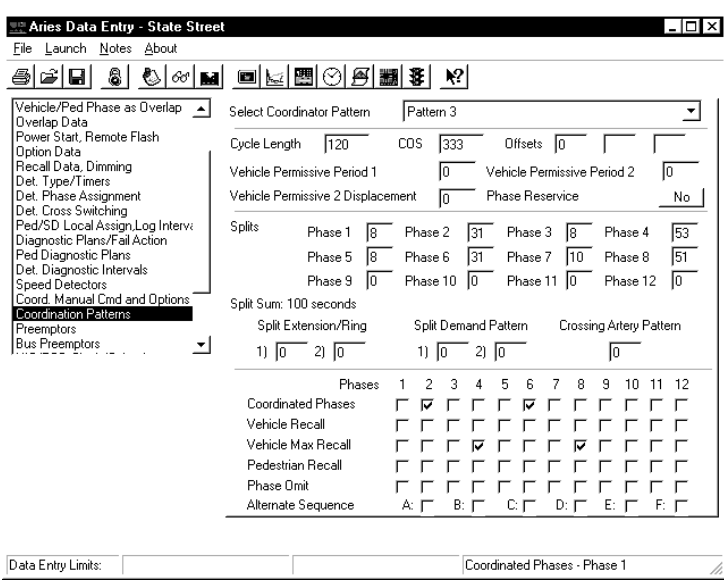

(b) Aries

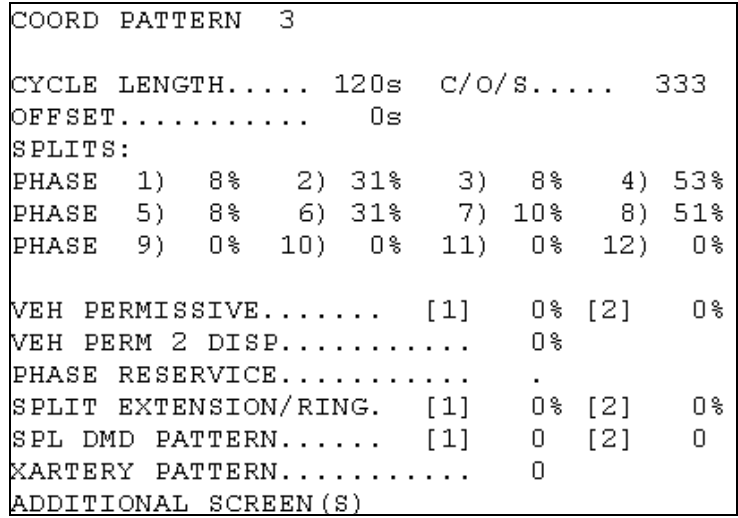

\begin{tabular}{|c|c|c|c|c|c|c|c|c|c|c|}
\hline PHASE & 12 & 3 & 4 & .5 & 6 & 7 & 8 & 9 & 0 & $\begin{array}{l}1 \\
1\end{array}$ \\
\hline COORD PHASES & . $\mathrm{x}$ & . & . & . & $x$ & . & . & . & ' & . \\
\hline VEHICLE RECALL & . & . & . & . & - & - & . & . & & \\
\hline VEH MAX RECALL & . & . & $\mathrm{X}$ & . & . & - & $\mathrm{x}$ & ${ }^{\circ}$ & & \\
\hline PED RECALL & . & . & . & . & . & . & . & . & . & \\
\hline PHASE OUIT & . . & . & . & . & . & . & . & . & . & . \\
\hline SPARE & . . & . & . & . & . & . & . & . & ' & . \\
\hline & A $B$ & $\mathrm{C}$ & D & $\mathrm{E}$ & $\mathrm{E}$ & & & & & \\
\hline ALT GEQUENCE: &. & . & . & . & . & & & & & \\
\hline
\end{tabular}

(c) Controller (MM, 3, 4, PgDn(4))

Figure 3-42 Coordination Pattern 3 


\subsection{Coordination Minimum Green}

When running a coordinated system, the minimum green times may have to be recalculated.

Consider phase 1 with a minimum green time of 5 seconds. Compare this number to the shortest green time in the coordination patterns for this phase. Since the splits are percentages, the lowest percentage does not indicate the shortest split in seconds since the cycle lengths vary. The shortest split for phase 1 is 9.6 seconds ( $8 \%$ of 120 seconds) from coordination pattern 3 in Figure 3-42. After the shortest split time is determined, the minimum green time can be calculated by subtracting the yellow and red clearance times.

Phase Minimum Green $=$ Shortest Split $(\mathrm{s})-$ Yellow - Red

Phase1 Minimum Green $=(8 \% * 120 \mathrm{~s})-3.2 \mathrm{~s}-2.0 \mathrm{~s}=4.4 \mathrm{~s} \leq 5.0 \mathrm{~s} !$

Since the minimum green time required for coordination is less than the minimum green previously entered, either the minimum green must be reduced or the split increased. Both Aries and the local controller will display an error when the coordination plans are entered and the minimum times are too high. The error messages for both are shown in Figure 3-43.

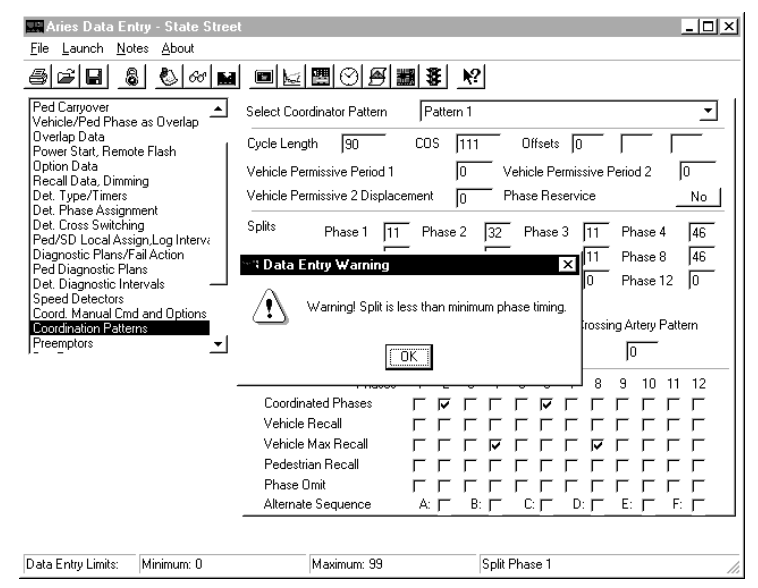

(a) Aries

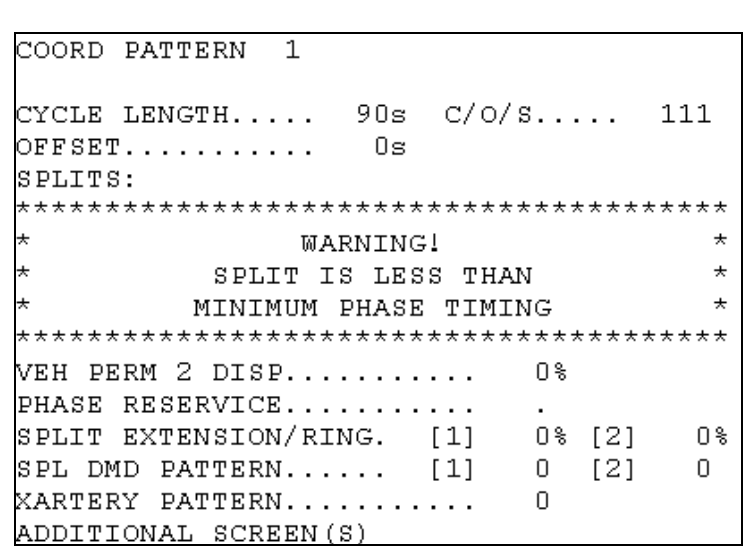

(b) Controller

Figure 3-43 Minimum Green Error

\subsection{Master Procedure}

\section{Main Menu $\rightarrow$ 1. Data Entry $\rightarrow 1$. System Parameters}

The cycle lengths for each coordination plan must be entered into the master controller. The master controller times its own cycle when a coordination plan is active. At master zero in the master cycle, sync pulses are sent to the local controllers. This ensures that the offsets are all 
referenced from the same time. For each local controller coordination plan, the cycle length must be entered into the master in the screen shown in Figure 3-44. Since only three coordination plans are being used in this example, only the first three entries need to be modified. If the cycle lengths are not entered, the local controllers will never be in coordination and coordination errors will be reported.

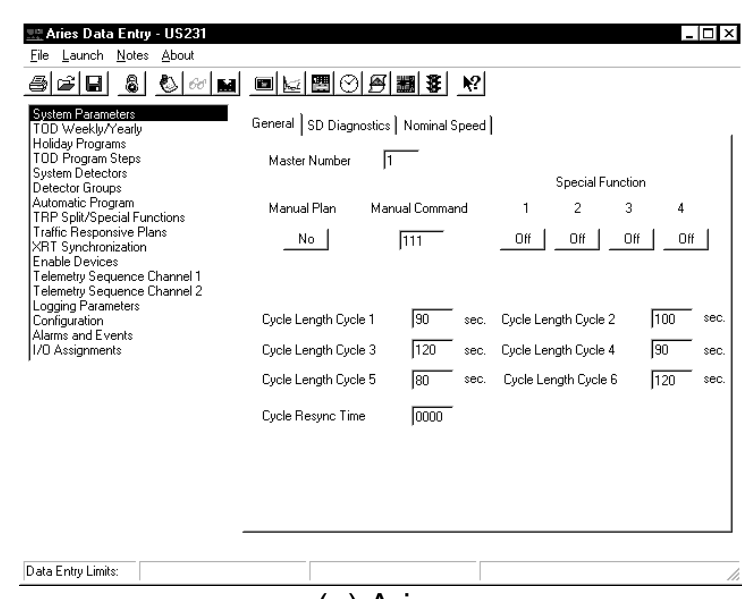

(a) Aries

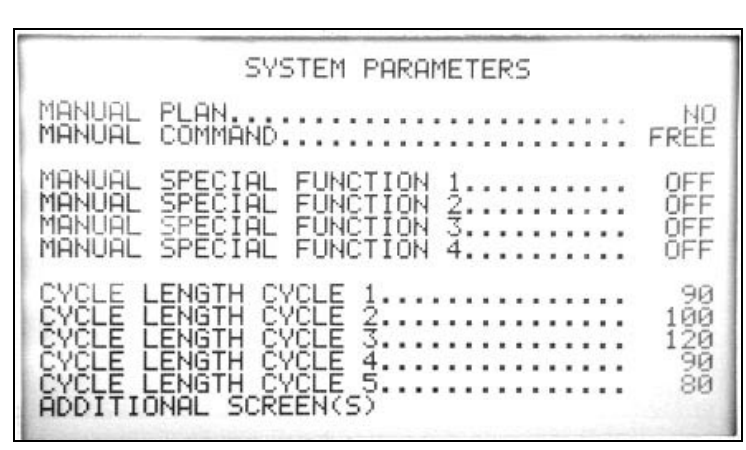

(b) Controller (MM, 1, 1, PgDn)

Figure 3-44 Master Cycle Length

\subsubsection{Time-of-Day Programs}

Programs to schedule particular timing plans can be created in both the master and the local controller. The programs can be run at a certain time of the day, day of the week, or day of the year. Common program purposes include running a certain coordination pattern, using a different max time, and turning on or off detector logging. In the master, a Time-of-Day (TOD) program is used to run certain coordination patterns in the system. In the local controller, a Non-Interconnect (NIC) program is used to run certain coordination patterns, while a TOD program is used to enable logging and switch max times. It is important to understand the difference between TOD and NIC. If the local controller is configured correctly, it will run the coordination pattern that the master TOD program indicates. The local controller also runs its own TOD programs that correspond to the program in effect. If the signal from the master is lost, the local controller will revert to its NIC programs to select the coordination patterns. The local controller can also override the master TOD program with its own NIC program under certain circumstances. The precedence of calling the programs is summarized in Table 3-2. 
Table 3-2 INDOT Time of Day Precedence

\begin{tabular}{|l|c|c|c|}
\hline Parameter & Master TOD & Local NIC & Local TOD \\
\hline Purpose & $\begin{array}{c}\text { Selecting coordination } \\
\text { plans }\end{array}$ & $\begin{array}{c}\text { Selecting coordination } \\
\text { plans }\end{array}$ & $\begin{array}{c}\text { Enables/Disables } \\
\text { features such as Max2, } \\
\text { logging, and phase recall }\end{array}$ \\
\hline Status & $\begin{array}{c}\text { Always active, unless } \\
\text { overridden by NIC in } \\
\text { local controller or } \\
\text { during a coordination } \\
\text { error }\end{array}$ & $\begin{array}{c}\text { Only active if selected to } \\
\text { override the Master TOD } \\
\text { in the local controller or } \\
\text { during a coordination } \\
\text { error }\end{array}$ & $\begin{array}{c}\text { Always active, program } \\
\text { number must match } \\
\text { Master TOD/Local NIC } \\
\text { program number in effect }\end{array}$ \\
\hline
\end{tabular}

Table 3-3 shows the time-of-day schedule for this system. It is recommended to have at least 4 patterns for weekdays and 2 patterns for the weekends. Since the same sequence of patterns is active Monday through Friday, they will all be assigned to TOD Program 1. Program 1 runs 4 coordination patterns and free mode. Please note that it is not typical to have the same pattern running at both the morning and evening peak hours because the traffic patterns are rarely similar. Saturday and Sunday have the same sequence, so their steps will be assigned to program 2. It consists of 2 coordination patterns and free mode.

Table 3-3 Time of Day Program Schedule

\begin{tabular}{|c|c|c|c|c|c|c|c|}
\hline Day of Week & Sun & Mon & Tues & Wed & Thu & Fri & Sat \\
\hline TOD Program & 2 & 1 & 1 & 1 & 1 & 1 & 2 \\
\hline 6am-9am & \multirow{4}{*}{$2 / 2 / 2$} & $3 / 3 / 3$ & $3 / 3 / 3$ & $3 / 3 / 3$ & $3 / 3 / 3$ & $3 / 3 / 3$ & \multirow{4}{*}{$2 / 2 / 2$} \\
\hline 9am-3pm & & $2 / 2 / 2$ & $2 / 2 / 2$ & $2 / 2 / 2$ & $2 / 2 / 2$ & $2 / 2 / 2$ & \\
\hline 3pm-6pm & & $3 / 3 / 3$ & $3 / 3 / 3$ & $3 / 3 / 3$ & $3 / 3 / 3$ & $3 / 3 / 3$ & \\
\hline 6pm-10pm & & $1 / 1 / 1$ & $1 / 1 / 1$ & $1 / 1 / 1$ & $1 / 1 / 1$ & $1 / 1 / 1$ & \\
\hline 10pm-6am & Free & Free & Free & Free & Free & Free & Free \\
\hline
\end{tabular}

\subsection{Master Procedure:}

The master TOD programs should match the local NIC programs. Using the master to activate coordination plans is the best way to ensure that all local controllers in the system are running the same plan. If the local controllers do not run the same plans, the system is not coordinated.

1. The step number is not a crucial number. The TOD step that is active is shown in the status display screen so that the programmer can reference the plan in the TOD menu to see what parameters are enabled.

2. The program number is very important. Step numbers that have common program numbers will run together. In Figure 3-45a \& b, step 1 is assigned to program 1 and enables coordination pattern 333 at 6:00 am. Notice that the coordination pattern is called according to its cycle/offset/split and not its coordination pattern number. In 
addition, steps 2-5 are assigned to program 1. Steps 6 and 7 are assigned to program 2 . Entering a 0 for the c/o/s enables the intersection to run free.

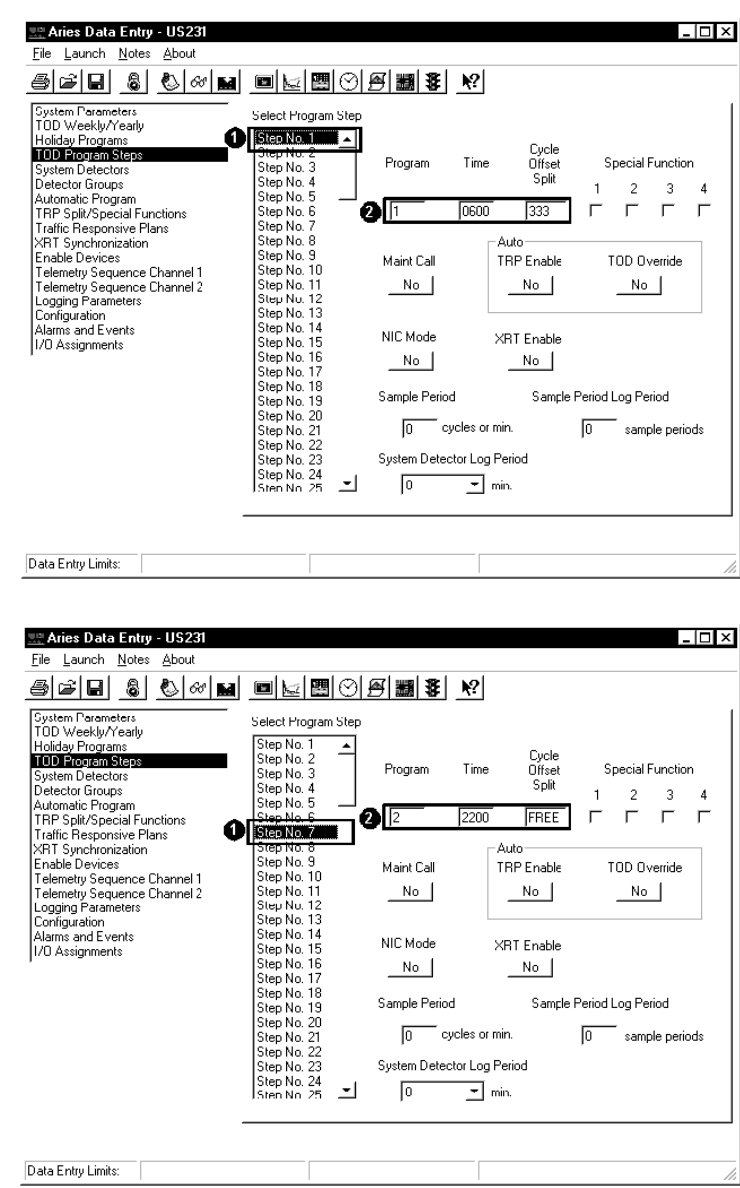

(a) Aries Steps 1 and 7

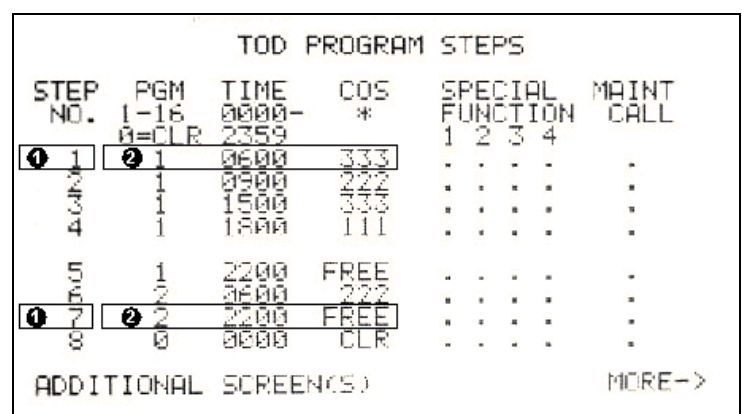

(b) Controller Steps (MM, 1, 4)

Figure 3-45 Master Time-of-Day Steps

3. Programs can be enabled on a certain day of the week. In Figure 3-46a \& b, program 1 runs Monday - Friday and program 2 runs on Saturday and Sunday. The week program number can be enabled based on the week of the year in the menu shown in Figure $3-46 c \& d$. 


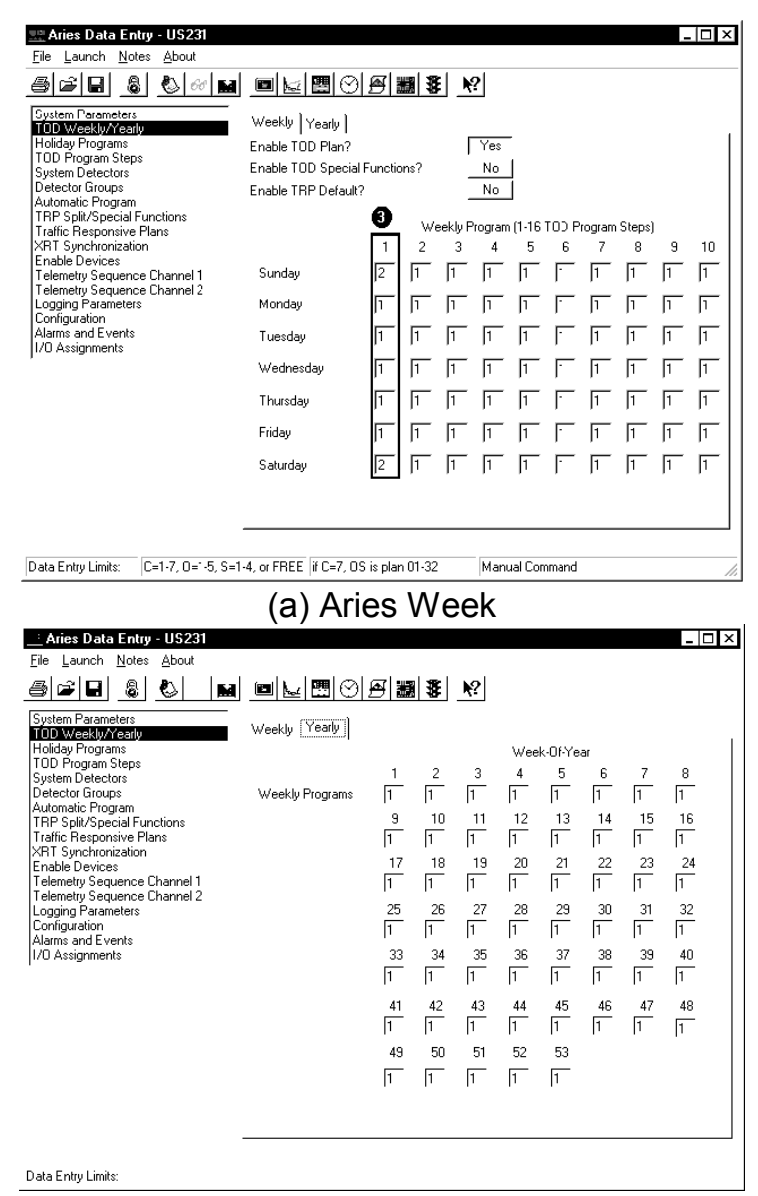

(c) Aries Year

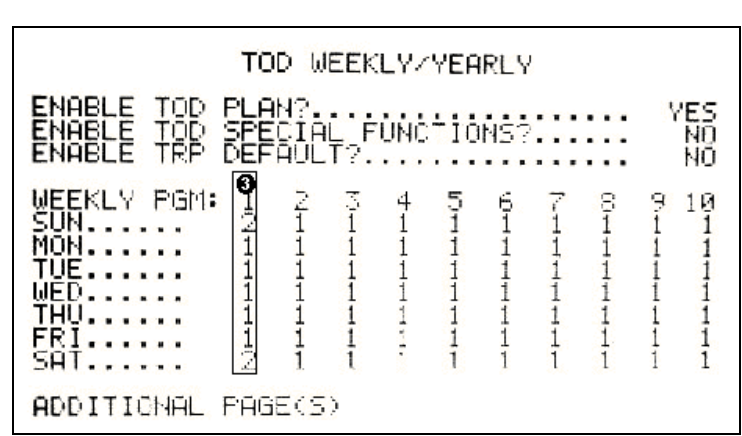

(b) Controller Week (MM, 1, 4)

\begin{tabular}{|c|}
\hline TOD MEEKL $Y$ Y YEARLY \\
\hline $\begin{array}{llllllll}1 & 2 & 3 & 4 & 5 & 6 & 7 & 8 \\
1 & 1 & 1 & 1 & 1 & 1 & 1 & 1\end{array}$ \\
\hline $\begin{array}{cccccccc}9 & 1 & 10 & 12 & 15 & 14 & 15 & 16 \\
1 & 1 & 1 & 16 & 1 & 1 & 1\end{array}$ \\
\hline 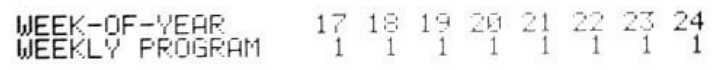 \\
\hline 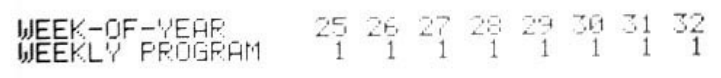 \\
\hline $\begin{array}{l}\text { MEEK -OFF-UEAF } \\
\text { AODITIOHAL SCFEEHE, }\end{array}$ \\
\hline
\end{tabular}

(d) Controller Year (MM, 1, 4, PgDn)

Figure 3-46 Master Time-of-Day Week and Year Plans

\subsection{Local Procedure:}

\subsection{NIC Procedure}

The NIC program should match the TOD program in the master. In the event that communication is lost to the master, the local controller will still be running the same plan as the rest of the system. If override is selected for a step, the pattern in that step will used instead of the one indicated by the master. Just like the Master TOD steps, the NIC steps are called according to their program number. In Figure 3-47b, there are two programs 1 and 2. In Figure 3-47d, the programs are called on different days of the week. Program 1 runs Monday - Friday and program 2 runs on Saturday and Sunday in weekly program 1 . Weekly program 1 is the only one used here, but they can be enabled according to the week of the year, just like the Master TOD plans.

1. The step number here is handled exactly as it was with the master TOD. It is not significant, only used to reference the particular step on the status display. 
2. The program number must correspond to the program that the step is in. Figure 3-47a \& b, step 1 is assigned to program 1 and it starts at 5:00am. The pattern here is referenced by the coordination pattern number, not the cycle/offset/split.

3. The weekly and yearly programs are identical to the ones shown for the Master TOD in Figure 3-46. The program numbers used here correspond to both the NIC programs and the local TOD programs.

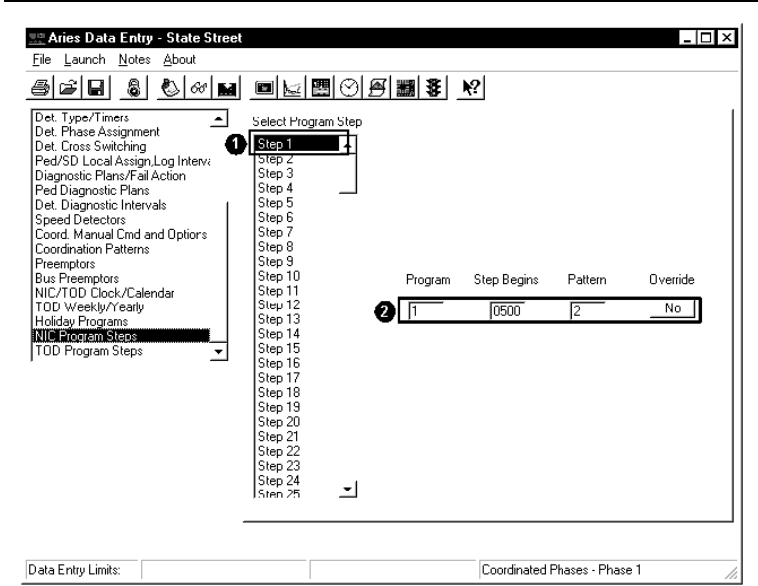

(a) Aries NIC Step 1

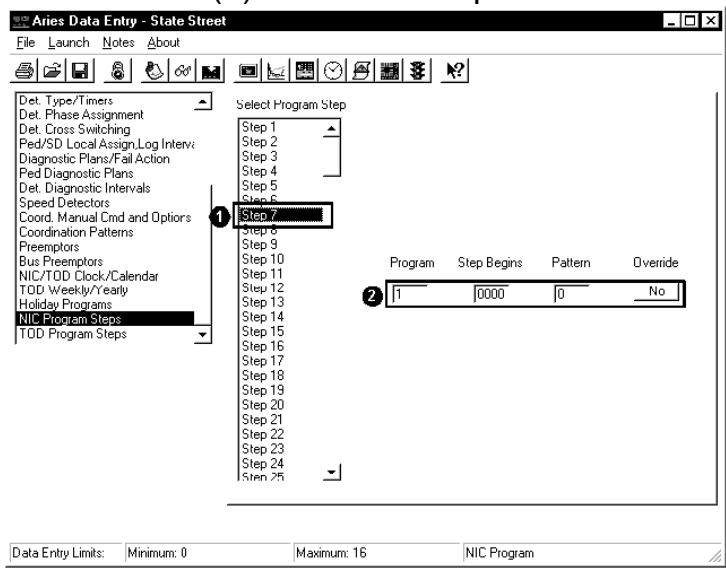

(c) Aries NIC Step 7

\begin{tabular}{|c|c|c|c|}
\hline \multirow[b]{2}{*}{ STEP } & \multicolumn{3}{|c|}{ NIC PROGRAM STEP } \\
\hline & PGM TIME & PATTERN & OVERRIDE \\
\hline (1) 1 & $125: 00$ & 2 & . \\
\hline 2 & $7: 00$ & 3 & . \\
\hline 3 & $110: 00$ & 1 & . \\
\hline 4 & $112: 00$ & 2 & . \\
\hline 5 & $115: 00$ & 3 & . \\
\hline 6 & 1 19:00 & 2 & . \\
\hline 7 & $0: 00$ & 0 & . \\
\hline 8 & $5: 00$ & 1 & . \\
\hline 9 & 9:00 & 2 & . \\
\hline 10 & $0: 00$ & 0 & . \\
\hline 11 & $0: 00$ & 0 & . \\
\hline 12 & $0: 00$ & 0 & . \\
\hline \multicolumn{4}{|c|}{ ADDITIONAL SCREEN (S) } \\
\hline
\end{tabular}

(b) Controller NIC Step 1 (MM, 5, 4)

\begin{tabular}{|ccccc|}
\hline \multicolumn{5}{c|}{ NIC RROGRAM STEP } \\
STEP & PGM & TIME & PATTERN & OVERRIDE \\
1 & 1 & $5: 00$ & 2 &. \\
2 & 1 & $7: 00$ & 3 &. \\
3 & 1 & $10: 00$ & 1 &. \\
4 & 1 & $12: 00$ & 2 &. \\
5 & 1 & $15: 00$ & 3 &. \\
6 & 1 & $19: 00$ & 2 &. \\
\hline 7 & 1 & $\mathbf{2} 0: 00$ & 0 &. \\
\hline 8 & 2 & $5: 00$ & 1 &. \\
9 & 2 & $9: 00$ & 2 &. \\
10 & 2 & $0: 00$ & 0 &. \\
11 & 0 & $0: 00$ & 0 &. \\
12 & 0 & $0: 00$ & 0 &. \\
ADDITIONAL & SCREEN (S) \\
\hline
\end{tabular}

(d) Controller NIC Step 7 (MM, 5, 4)

Figure 3-47 Local Non-Interconnect Configuration

\subsection{TOD Procedure}

The local TOD steps run concurrently with the master TOD or local NIC plans that are in effect. The most common applications of the local TOD is to turn on and off detector logging and enable alternative max green times at certain times. The TOD steps shown in Figure 3-48 are used to start logging detector data. Data at this intersection will be logged all week. More details about detector logging will be discussed in the next section. 
1. The step number is insignificant. It is only used to reference the step in the status display.

2. The program number used here should correspond to the NIC program that it will run with. In this case, we are logging detector data every day. The NIC programs that are active on these days are programs 1 and 2 . Therefore, the TOD steps can be assigned to program 1 or 2 . In Figure 3-46, one step in program 1 enables logging at 6:00. This step will remain active at all times since it is never turned off. Another step would have to be created to stop the detector logging, if desired.

3. In step 1, the detector logging is enabled with an ' $x$ '.

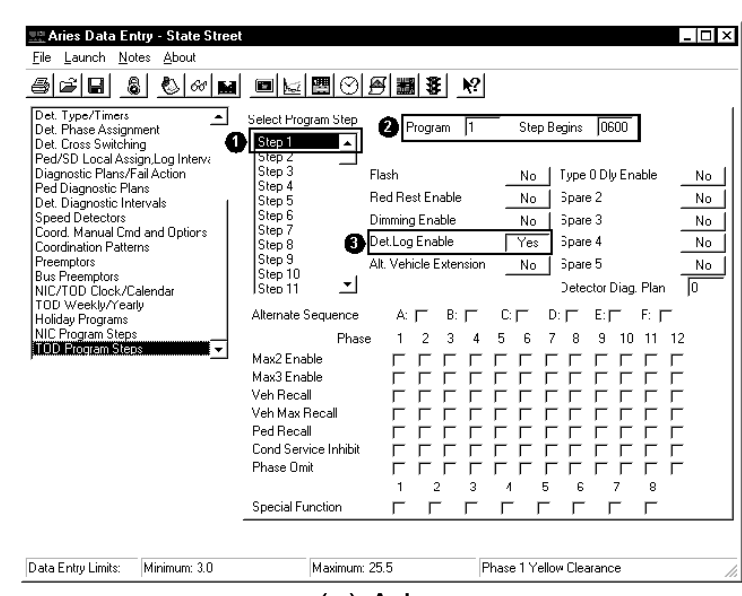

(a) Aries

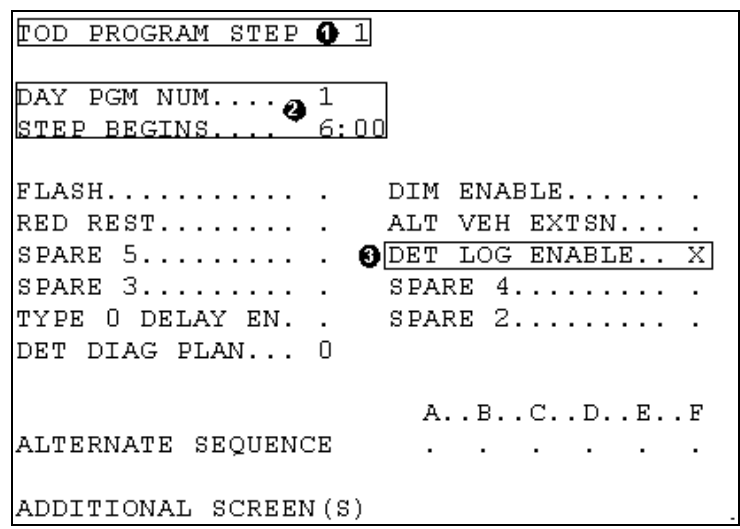

(b) Controller (MM, 5, 6)

Figure 3-48 Local Time-of-Day Configuration

\subsubsection{Detectors}

A typical INDOT system has all presence detectors. As discussed in Chapter 2, the common detection layout is $3-6^{\prime} \times 6^{\prime}$ loops wired together starting at the stop bar for presence and a fourth loop wired separately for counting. In Figure 3-49a, in the northbound left-left turn lane, detector 5 is a presence detector and detector 15 is a count detector for phase 5 shown earlier in Figure $3-22 a$. The numbering scheme used here is not the conventional one discussed earlier in Chapter 2 because this is being set up for TS2 Type 2 and was limited to 20 detectors.

\subsection{Type and Assignment}

The count detectors are enabled for logging so the count data can be accessed with Aries. In Figure 3-50b, detectors 18 and 20 are assigned type 1 because a delay time has been entered. These two detectors are in a right-turn only lane. In this situation, when a car activates one of these detectors during the red phase, the call is delayed for ten seconds, in case the car turns right on red. If the car has not accepted a gap within ten seconds, the call is placed. The 
detector in the right-turn lane can also be a type 0 detector and the delay enabled with a TOD plan (Figure 3-48).

1. In Figure 3-49, the intersection detectors are assigned by number, to their corresponding phase by placing an ' $x$ ' in the corresponding row and column. For example, detectors 2 , 10 , and 18 are all assigned to phase 2 because that is the phase that they are called. Make sure that these detectors are not assigned to any other phase. There should only be one ' $x$ ' in each row. Detectors 12, 9, and 17 are also assigned to phases because they detect, as well as count. 


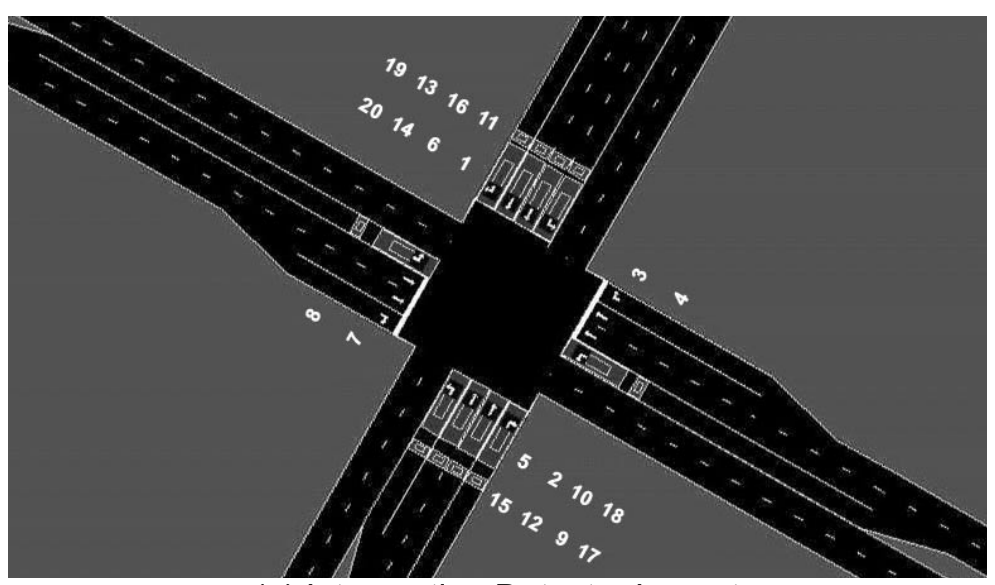

(a) Intersection Detector Layout

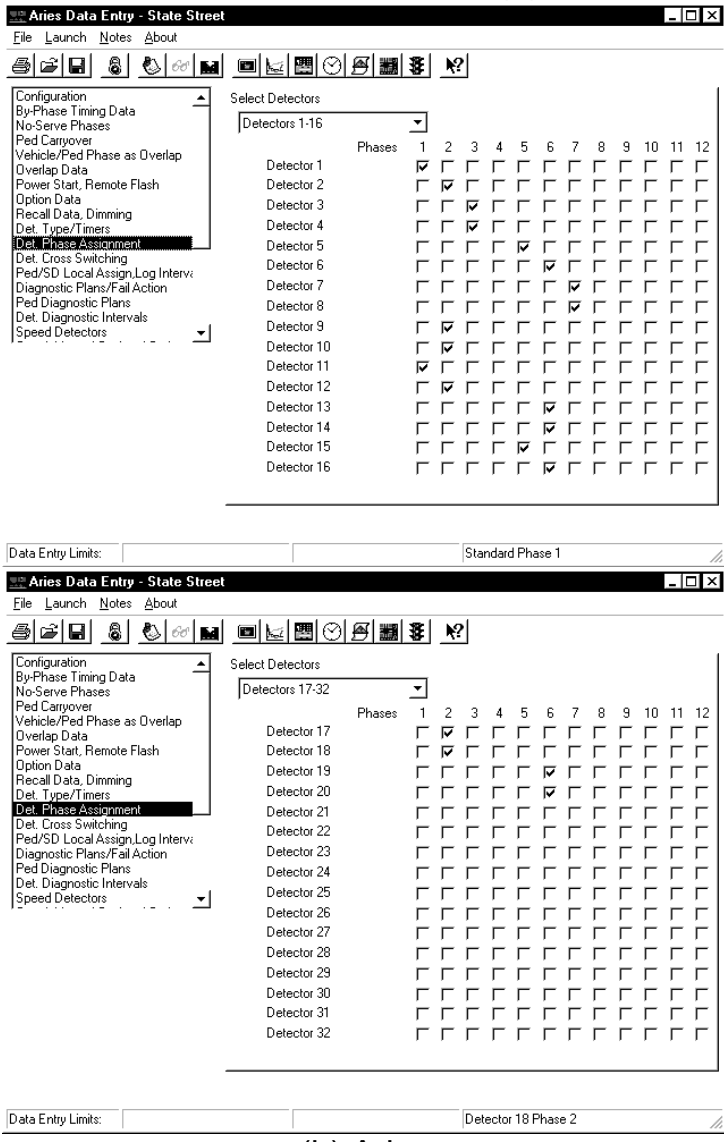

(b) Aries
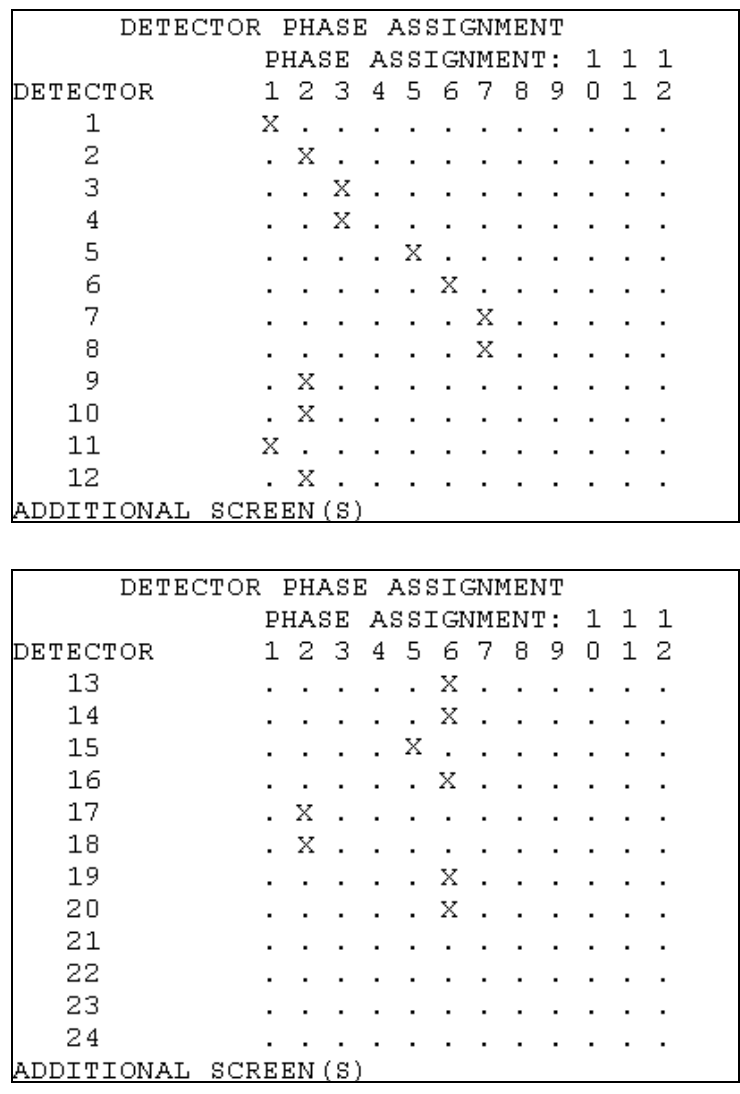

(c) Controller (MM, 6, 2)

Figure 3-49 Aries Detector Assignment

2. The detector types are assigned in Figure 3-50. All presence detectors at this intersection are normal detectors. However, the two presence detectors in the right-turn lanes, detectors 18 and 20 , are assigned type 1, which enables the extend and delay 
settings. The count detectors are all configured as normal detectors, but logging is enabled for them. Detector 12 in Figure $3-50 a$ \& $b$ is shown with logging enabled.

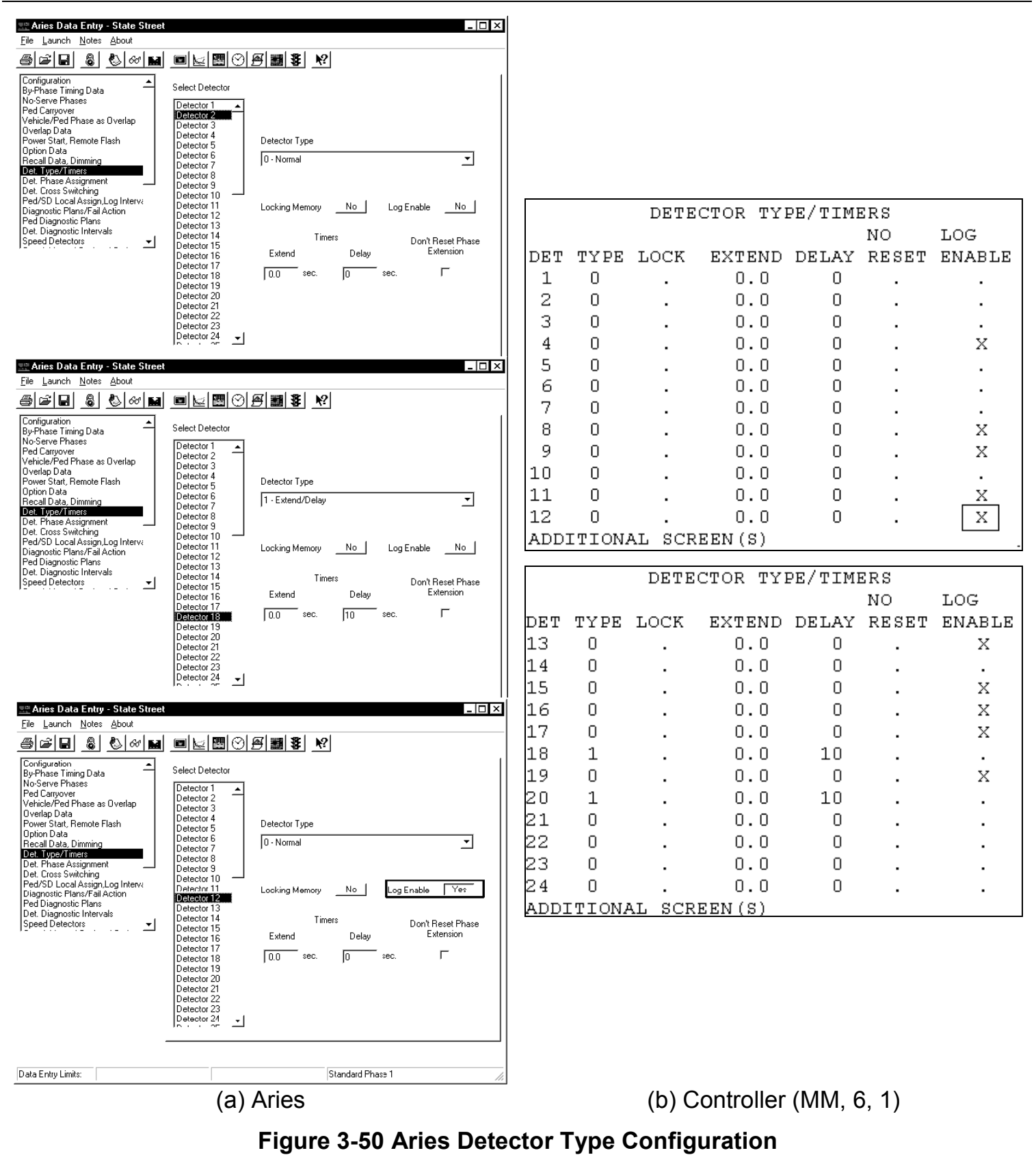

\subsection{Logging}

Installing count detectors allows the local controller to collect volume data. Since the count detectors are presence detectors, occupancy data will be collected. Logging of the count detectors was enabled previously in Figure 3-50 and enabled by a TOD program step in Figure 
3-48. The data that is logged cannot be viewed from the controller; it must be downloaded to Aries. In the local controller, the log interval needs to be configured. Interval choices are 5, 15, 30 , and 60 minutes. INDOT logs for 60 minutes to give hourly volumes because they are easily used for developing signal timing plans.

The data that is collected is stored in the controller memory buffer. This data should be downloaded on a regular basis, usually once a week. Once the buffer is full, previous log buffer data will be overwritten. The buffer has 20,000 bytes for storing the detector log data. For any logging interval, the size of the data collected can be calculated with the following equation:

Record Size $=19+(3 *$ \# of Volume/Occupancy Detectors $)+\#$ of Speed Detectors Eq. 3-2

Record Size $=19+(3 * 10)+0=49$ bytes

Once the record size is determined, the maximum number of logging hours can be calculated to determine how often data should be downloaded to avoid losing any data. Assuming a 60 minute logging interval and logging duration of 24 hours per day, the maximum number of days can be calculated as follows:

$$
\begin{aligned}
& \text { Maximum \# of Records }=\frac{\text { Maximum Memory Space }}{\text { Record Size }} \\
& \text { Maximum \# of Records }=\frac{20,000 \text { bytes }}{49 \text { bytes }}=408 \text { records } \\
& \text { Maximum \# of Days }=\# \text { of Records } * \frac{\text { Log Interval }}{60 * \text { Hours Per Day }} \\
& \text { Maximum \# of Days }=408 \text { records } * \frac{60 \text { minutes }}{60 * 24}=17 \text { days }
\end{aligned}
$$

Since the buffer can store 17 days worth of detector data at this intersection, if the data was transferred once every 2 weeks, none should be lost. 


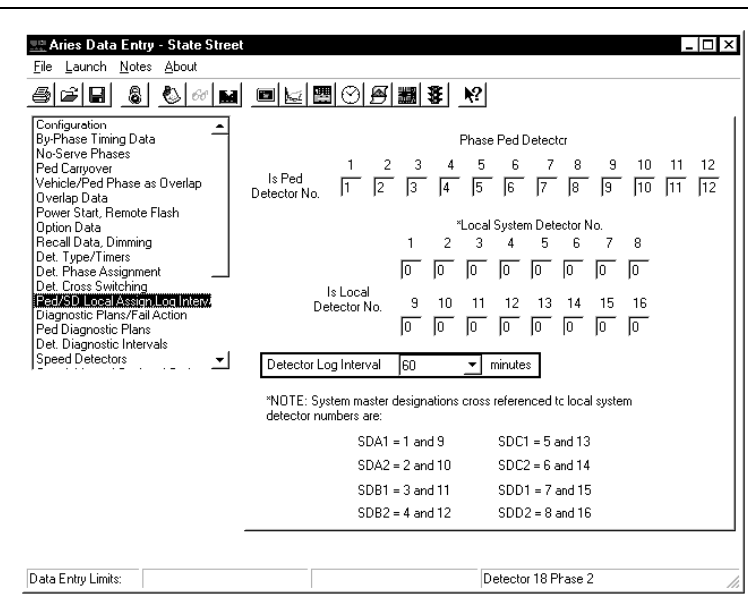

(a) Aries Log Interval

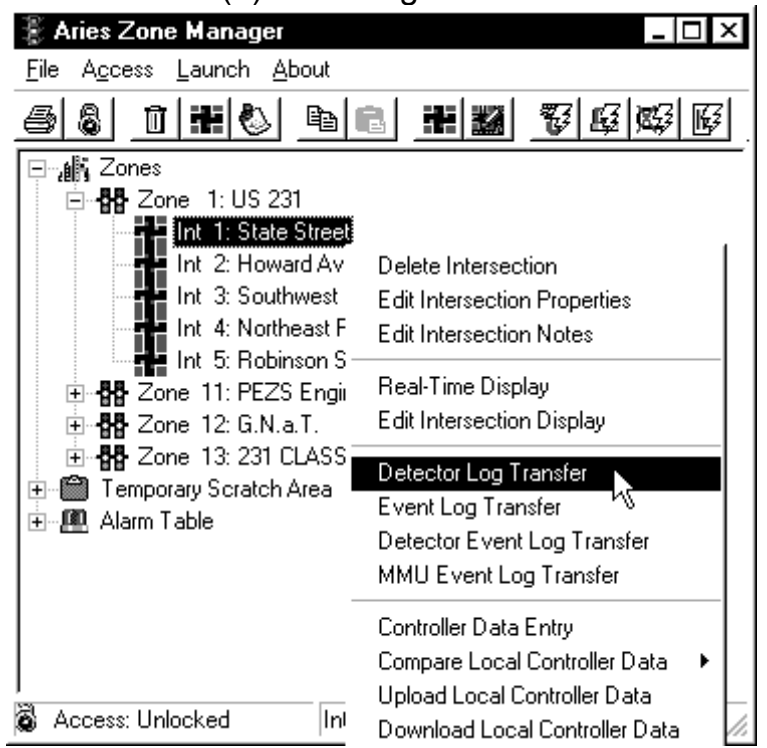

(c) Aries Download Detector Log

\begin{tabular}{|c|c|c|c|c|c|c|c|c|}
\hline \multirow{2}{*}{$\begin{array}{l}\text { PED AND } \\
\text { DETECTOR }\end{array}$} & \multirow{2}{*}{$\begin{array}{l}\text { SYSTEI } \\
\text { LOG }\end{array}$} & \multicolumn{4}{|c|}{ M DETECTOR LOCAL } & \multicolumn{3}{|c|}{ ASSIGNMENT } \\
\hline & & \multicolumn{4}{|c|}{ INTERVAL $\ldots \ldots \ldots$} & \multicolumn{3}{|c|}{60 MINUTES } \\
\hline \multicolumn{9}{|c|}{------------ - - - - - - - - - - - - - - - - - - - - - - } \\
\hline \multicolumn{2}{|l|}{ LOCAL } & \multicolumn{2}{|c|}{ - PHASE } & PED & \multicolumn{3}{|c|}{ DETECTOR - } & \\
\hline PED DET & & 1 & 2 & 3 & 4 & 5 & 6 & \\
\hline \multirow[t]{2}{*}{ NUMBER. . } & & 1 & 2 & 3 & 4 & 5 & 6 & \\
\hline & & 7 & 8 & 9 & 10 & 11 & 12 & \\
\hline NUMBER. . & & 7 & 8 & 9 & 10 & 11 & 12 & \\
\hline \multicolumn{9}{|c|}{ 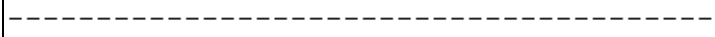 } \\
\hline LOCAL & -- & LOCAL & SY & STEM & DET & NUY & IBER & --- \\
\hline DETECTOR & 1 & 2 & 3 & 4 & 5 & 6 & 7 & 8 \\
\hline \multirow[t]{2}{*}{ NUMBER. . } & 0 & 0 & 0 & 0 & 0 & 0 & 0 & 0 \\
\hline & 9 & 10 & 11 & 12 & 13 & 14 & 15 & 16 \\
\hline NUMBER. . & 0 & 0 & 0 & 0 & 0 & 0 & 0 & 0 \\
\hline END OE SL & UBMEN & & & & & & & \\
\hline
\end{tabular}

(b) Controller Log Interval (MM, 6, 3)

\section{Figure 3-51 Detector Logging Configuration}

Once the log data is transferred (Figure 3-51), it can be viewed in two different formats or output to a text file. The two output formats, table and graph, are shown in Figure 3-52. Aries will store data for each detector in each system every day of the year. The counts can be viewed as the raw volumes or as flow rates. The volume tables and graphs show the number of cars counted in each interval. The flow rate is the number of cars converted to an hourly volume. Since the data is collected in 60 minute intervals, the flow rate will be the same as the volume. This is the data that is shown in the flow rate tables and graphs. 


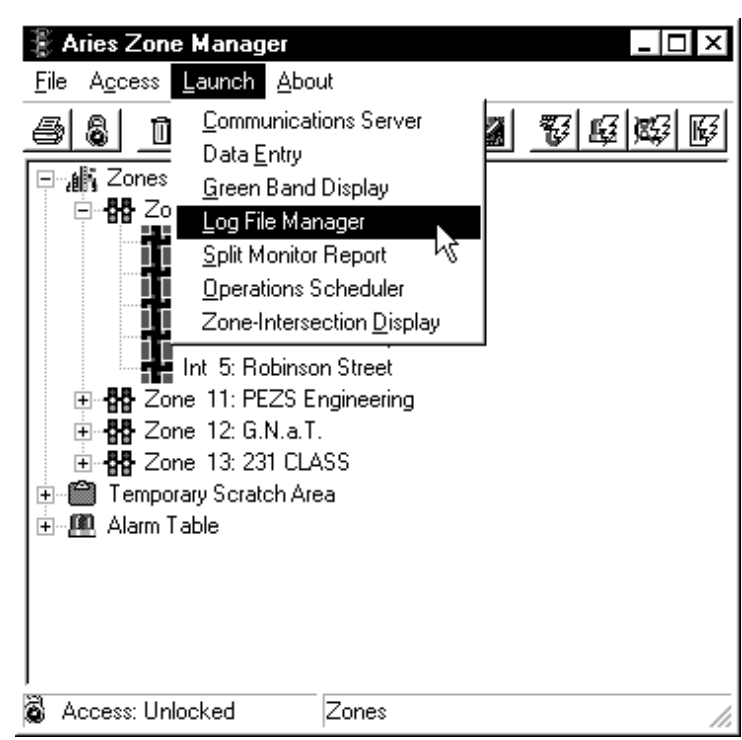

(a) Aries Log File Manager

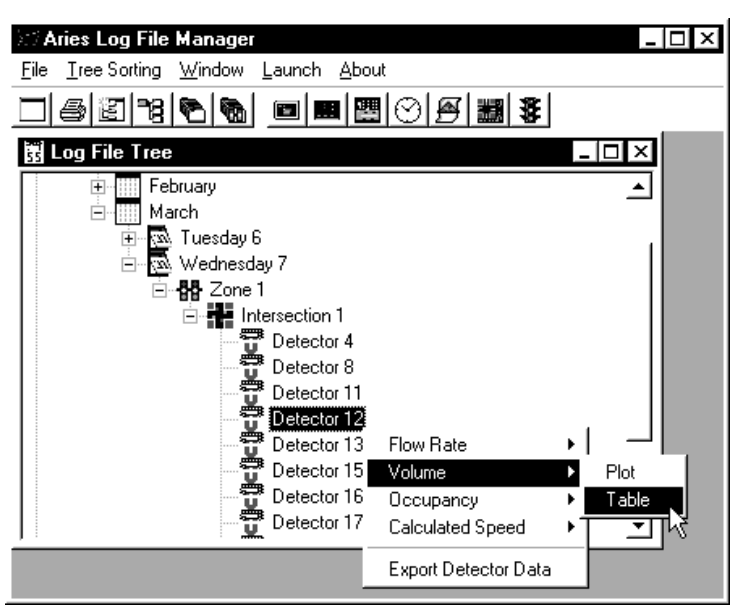

(b) Aries Log Output Selection

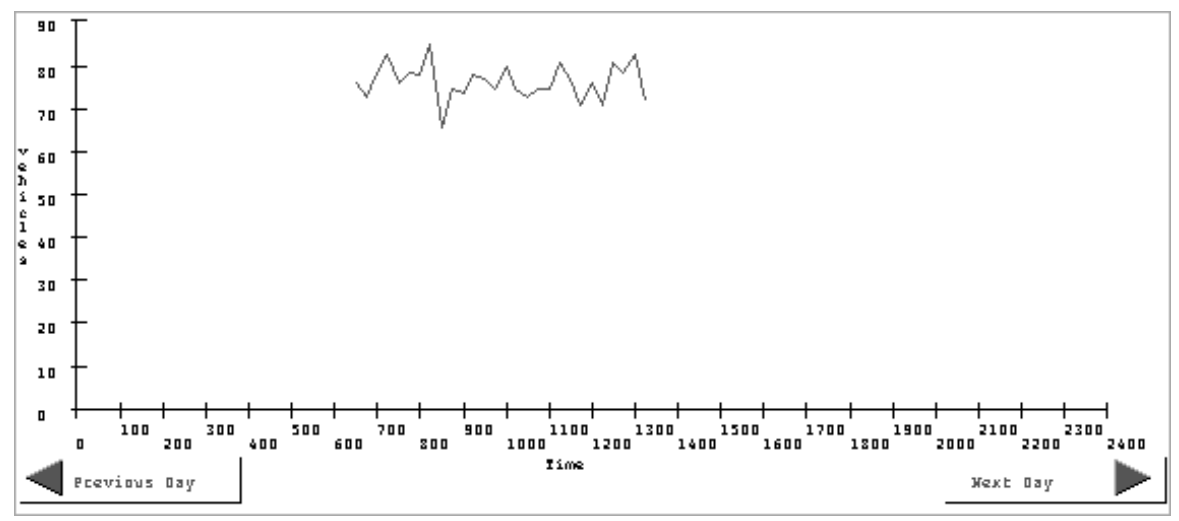

\begin{tabular}{|l|l|}
\hline 0600 & 73 \\
\hline 0615 & 77 \\
\hline 0630 & 75 \\
\hline 0645 & 72 \\
\hline 0700 & 78 \\
\hline 0715 & 82 \\
\hline 0730 & 75 \\
\hline 0745 & 78 \\
\hline
\end{tabular}

(c) Aries Volume Log Graph

\begin{tabular}{|l|l|}
\hline 0800 & 77 \\
\hline 0815 & 84 \\
\hline 0830 & 65 \\
\hline 0845 & 74 \\
\hline 0900 & 73 \\
\hline 0915 & 77 \\
\hline 0930 & 76 \\
\hline 0945 & 74 \\
\hline
\end{tabular}

\begin{tabular}{|l|l|}
\hline 1000 & 79 \\
\hline 1015 & 74 \\
\hline 1030 & 72 \\
\hline 1045 & 74 \\
\hline 1100 & 74 \\
\hline 1115 & 80 \\
\hline 1130 & 76 \\
\hline 1145 & 70 \\
\hline
\end{tabular}

\begin{tabular}{|l|l|}
\hline 1200 & 75 \\
\hline 1215 & 70 \\
\hline 1230 & 80 \\
\hline 1245 & 78 \\
\hline 1300 & 82 \\
\hline 1315 & 71 \\
\hline 1330 & 76 \\
\hline 1345 & 71 \\
\hline
\end{tabular}

(d) Aries Volume Log Table

Figure 3-52 Detector Logging Output

\subsubsection{Downloading To Controller}

Once all data has been entered into Aries, the database needs to be downloaded back to the controller. This needs to be done for both the master and the local controllers. This procedure can also be done with a batch operation, found in the Aries File menu (Figure 3-53). 


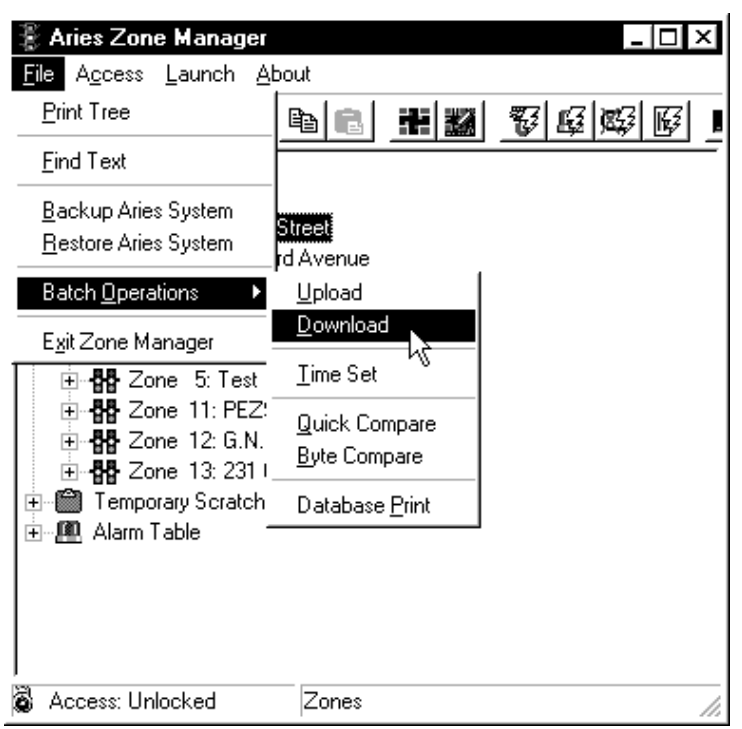

(a) Controller Batch Download
8 Aries Zone Manager

File Access Launch Bbbout
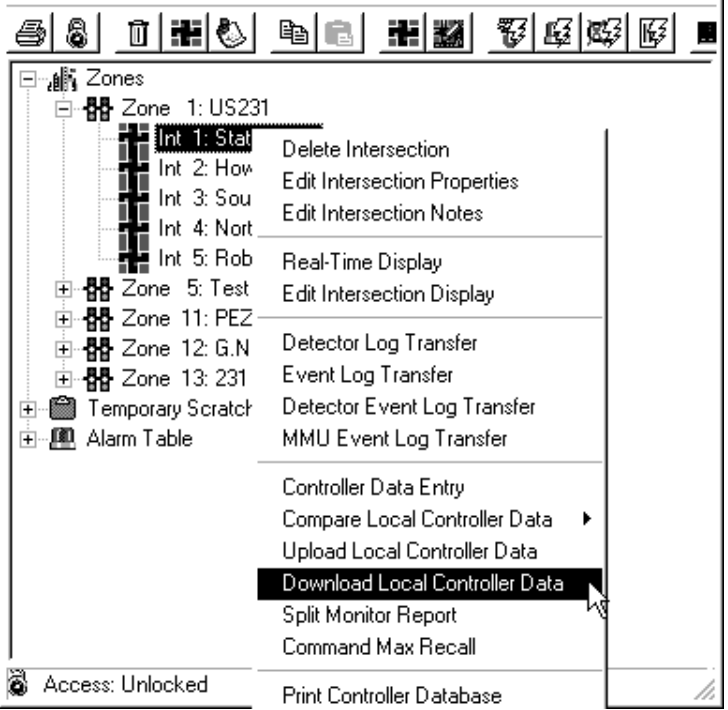

(b) Select Controller to Download

Figure 3-53 Download Databases

\subsubsection{Aries Graphics}

A helpful feature of Aries allows the user to see a graphic display of the controller state on the computer. Figure 3-54a \& b has a default graphic and a picture graphic for an arbitrary intersection. Figure $3-54 \mathrm{c}$ has a customized graphic for intersection 1 . In the Aries graphic editor, certain areas of the images can be assigned to a phase or a detector. The area will change colors when the state of that phase or detector changes. No assignments have to be made for the default graphics. Also, if an actual image of the intersection is going to be used like the one in Figure 3-54b, solid areas must be inserted into the picture using a graphic editor. Descriptions of the items in the custom graphics screen are:

1. The arrows are assigned to their corresponding phases. They will change color with the phase indications in the controller

2. The boxes behind each arrow are assigned to detectors in the controller. When the detector is active, the box changes color.

3. Messages can be inserted into the graphic that tell which coordination plan is in effect, the controller number, and the local time.

4. Other messages can be inserted that indicate the phase splits that are active, the offset, the overlap assignments, and the local cycle timer. 
Another assignment that can be made, but is not shown, is the pedestrian indications. Graphics can also be inserted that give information about the master. Different errors can also be reported in the real-time graphic display, such as coordination errors.

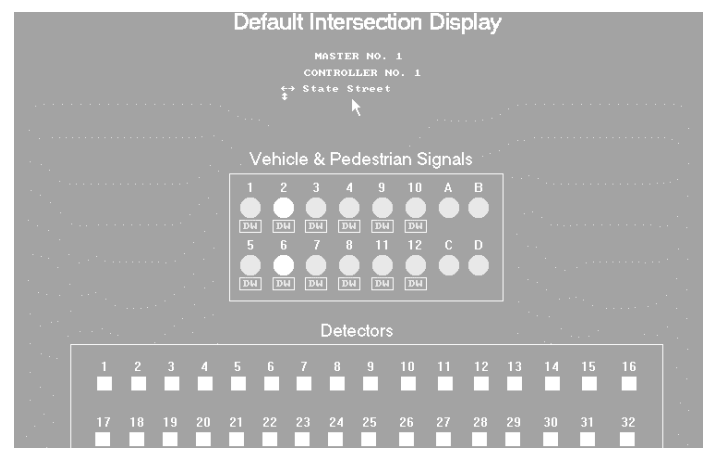

(a) Aries Default Graphics

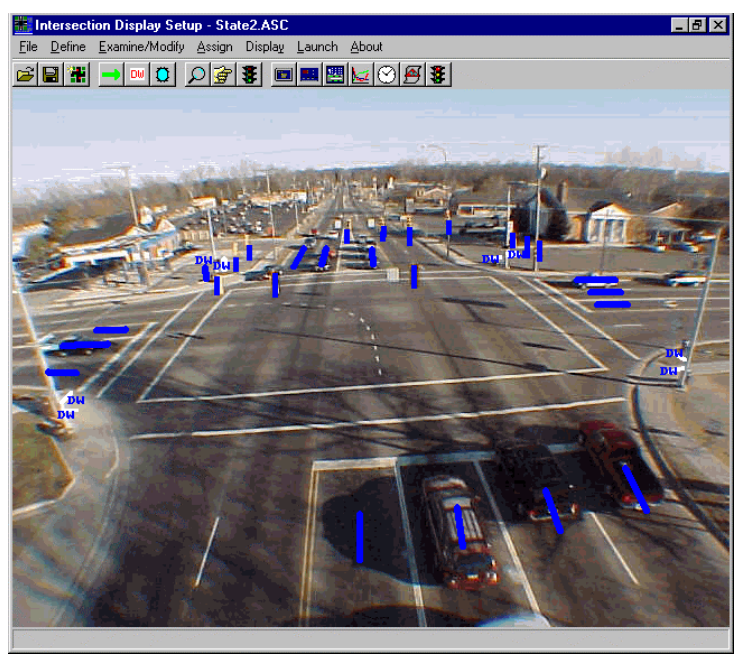

(b) Aries Custom Graphics

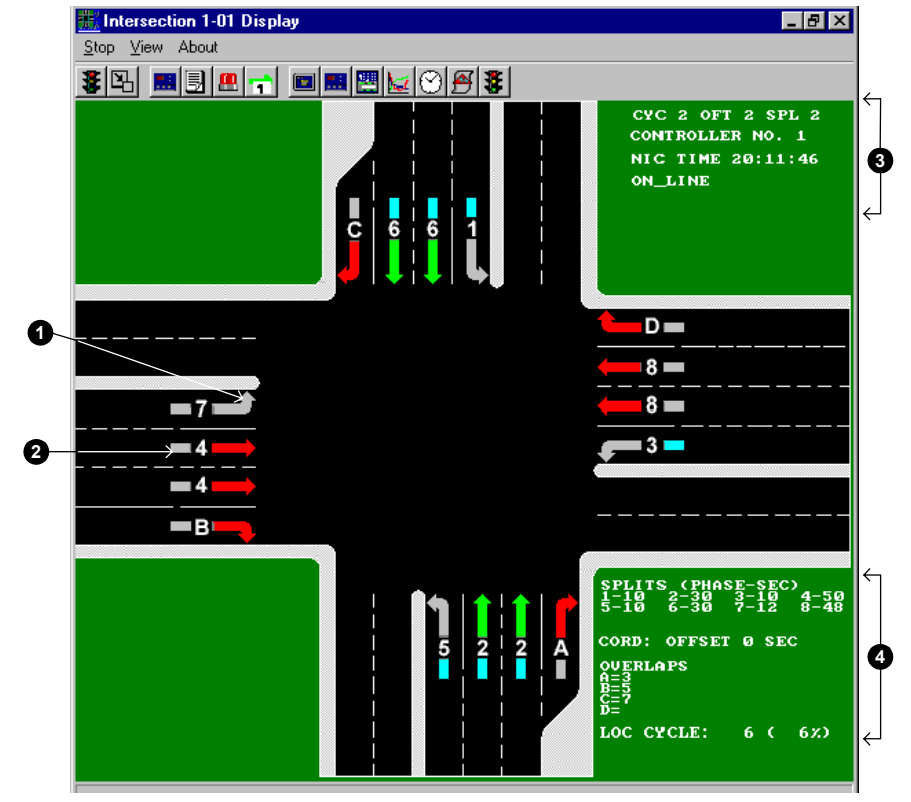

(c) Aries Custom Graphic for Intersection 1

Figure 3-54 Aries Graphics

\subsubsection{Greenband Analysis}

The greenband analysis provides the user with a time-space diagram like the one shown in

Figure 3-55. This allows the user to see the progression of vehicles through the system and 
makes it easier to visualize the effects of offsets. When the greenband analysis is launched, it takes a few cycles for the display to complete.

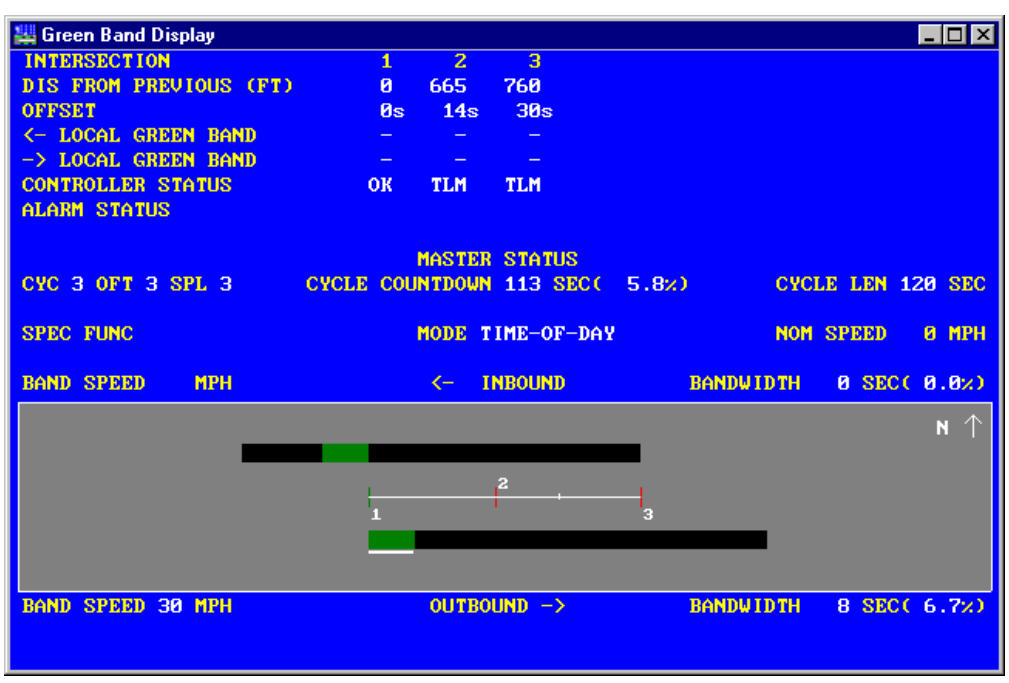

Figure 3-55 Aries Greenband Analysis 


\title{
Chapter 4. Configuring Peek Closed Loop Traffic Signal Systems
}

\author{
As was discussed in Chapter 1, the numerical design of parameters, such as the phase times and \\ coordination, is only part of the signal timing design. There are also a large number of vendor- \\ specific communication and system level parameters that must be understood and configured. \\ The purpose of Chapter 3 and this chapter is to show how to implement the data tabulated in \\ Chapter 2. Chapter 3 documented how to configure Econolite controllers to run a closed loop \\ system. This chapter will show, step-by-step, how to configure Peek controllers to implement a \\ closed loop system.
}

The signal design is discussed in Chapter 2. The next step is to enter this data into the controller and test it. The testing procedure is discussed in Chapter 5. The U.S. 231 system will continue to be used as an example, in particular, intersection 1. All U.S. 231 data can be found in Appendix B. Figure 4-1 shows the procedures that will be discussed in this chapter. 


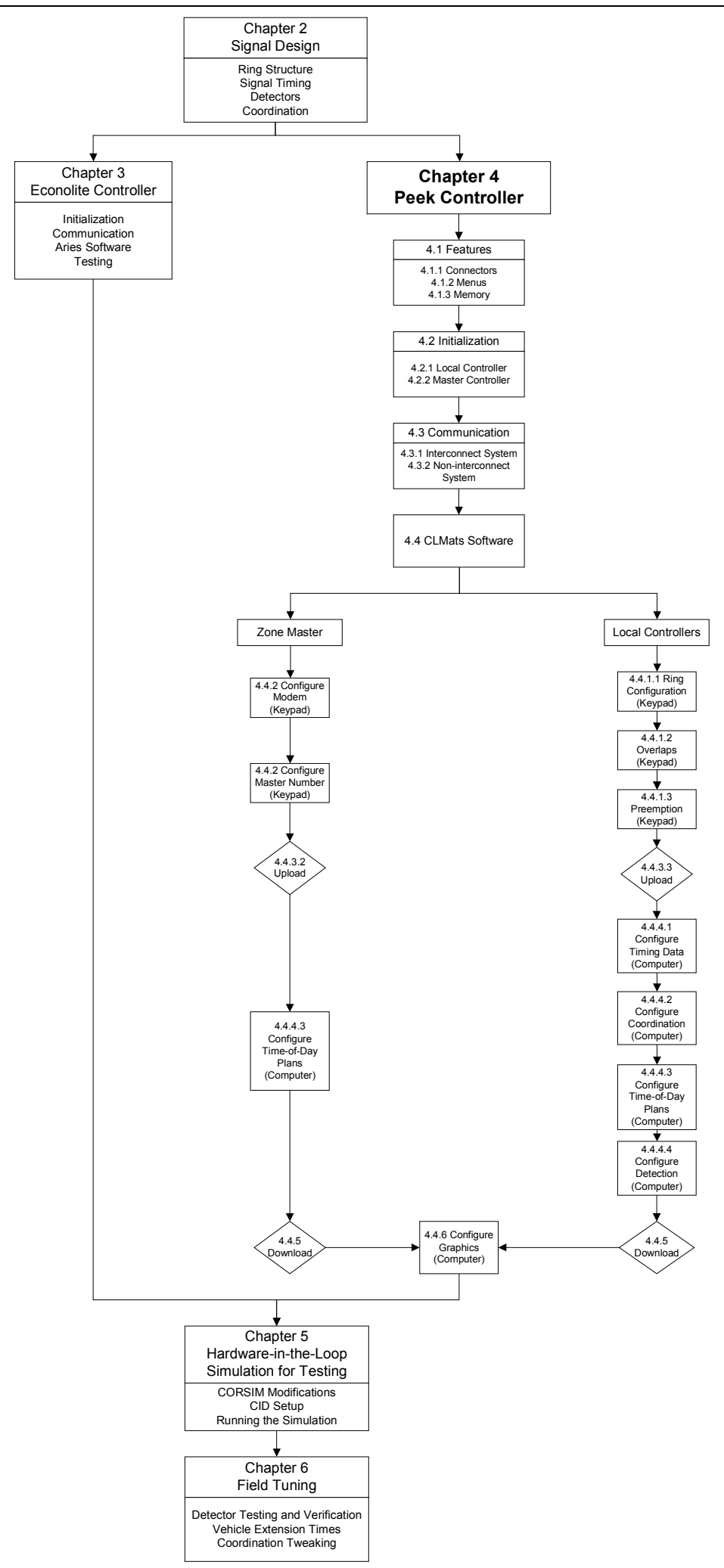

Figure 4-1 Chapter Concepts 


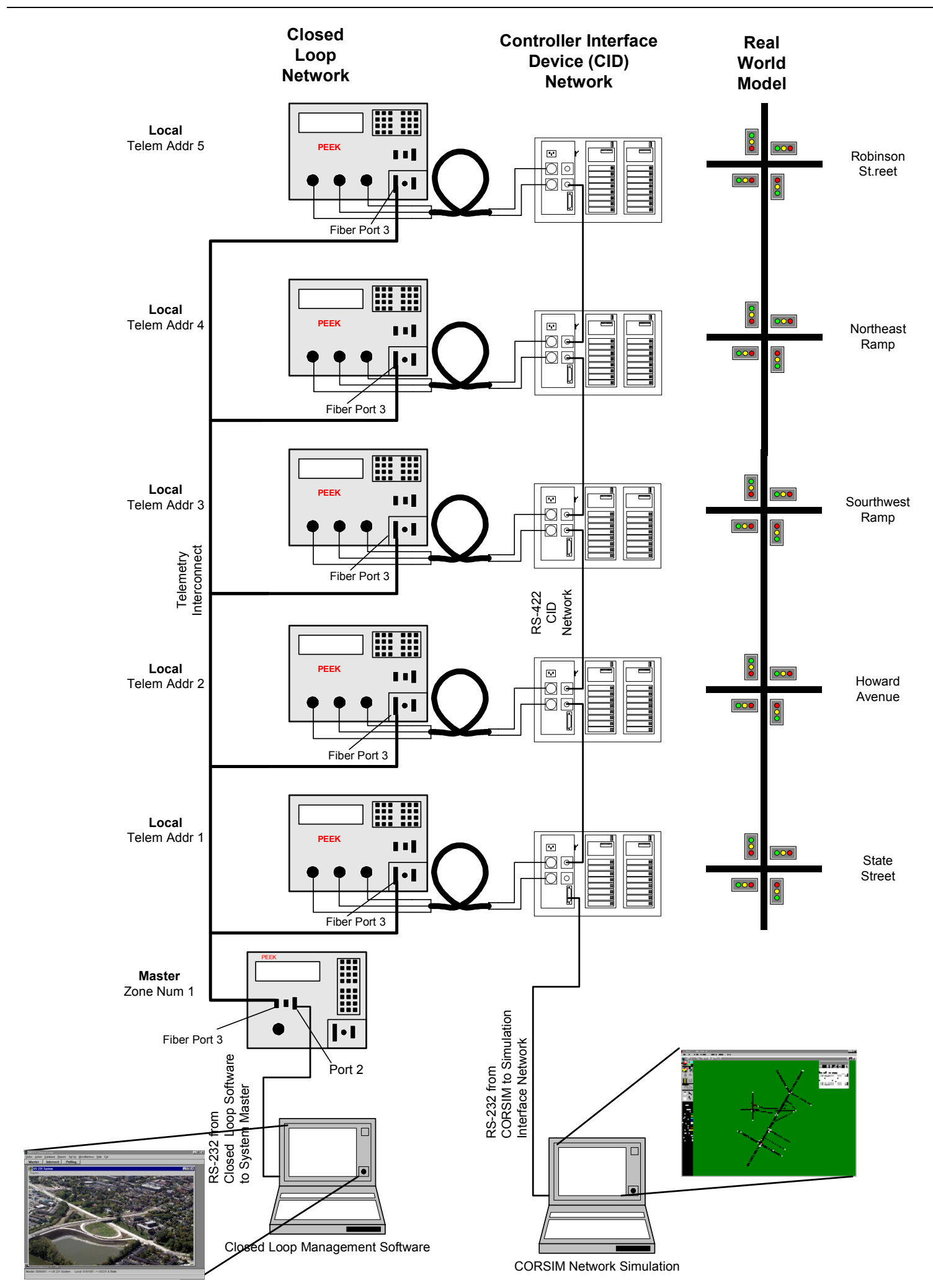

Figure 4-2 U.S. 231 Lab Setup 


\subsection{Controller Features}

\subsubsection{Connectors}

\subsubsection{Local Controller}

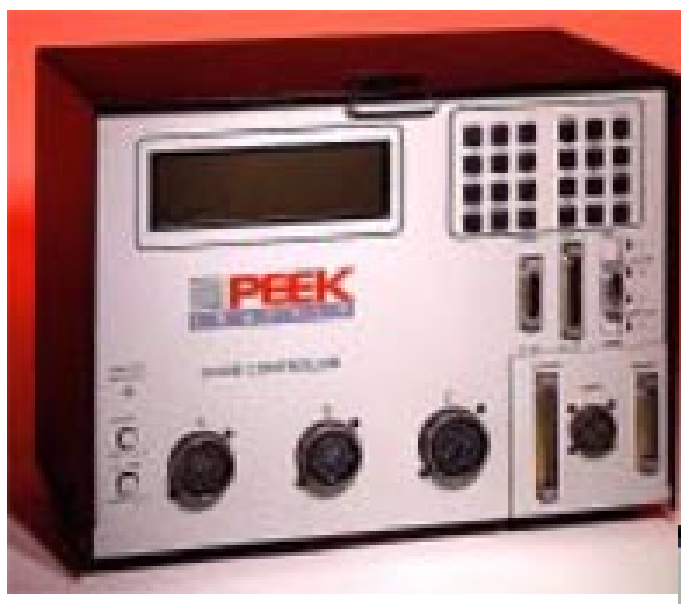

(a) Local Controller

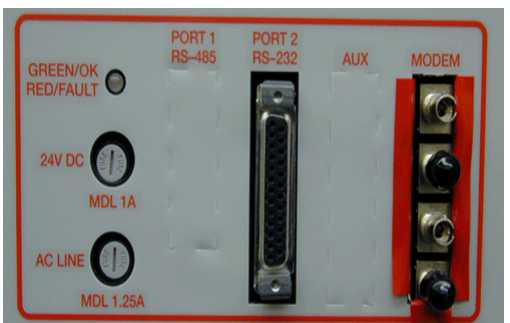

(b) Ports 1, 2, \& 3

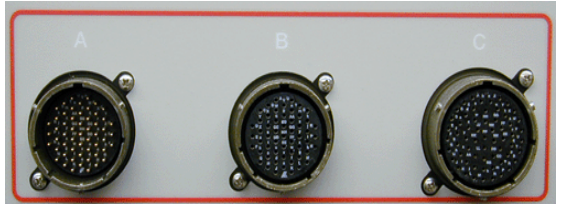

(c) NEMA A, B, C Connectors

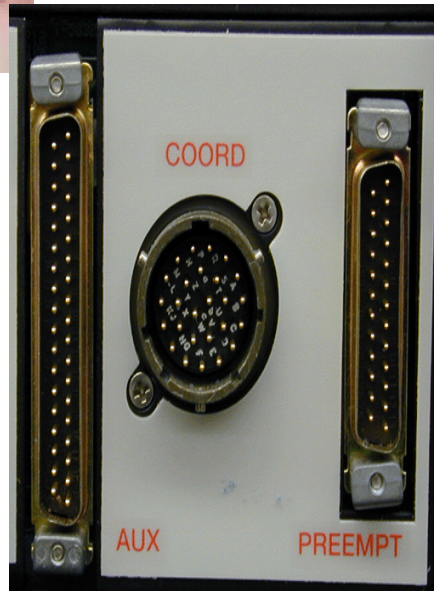

(d) Misc. Connectors

Figure 4-3 Local Controller and Connectors

The Peek local controller shown in Figure 4-3a has 6 connectors and 4 ports on its front panel. Connectors $\mathrm{A}, \mathrm{B}$, and $\mathrm{C}$ provide an interface to the signal cabinet or a controller interface device (cid) in a lab setting as shown in Figure 4-2. The auxiliary, coordination, and preemption connectors can also be used to interface with the signal cabinet. Port 1, or the synchronous data link control (SDLC), is used with Type 1 controllers to interface with the cabinet. In Type 1 cabinets, the SDLC takes the place of connectors A-D. Port 2 is used when directly connecting a computer or laptop to the controller to exchange data. When setting up an interconnected system with a master, port 3 (modem port) is used to communicate with the master via a telemetry link as 
shown in Figure 4-2. Specific pin assignments for the connectors and ports can be found in the Peek Controller Operating Manual. ${ }^{20}$

\subsubsection{Master Controller}

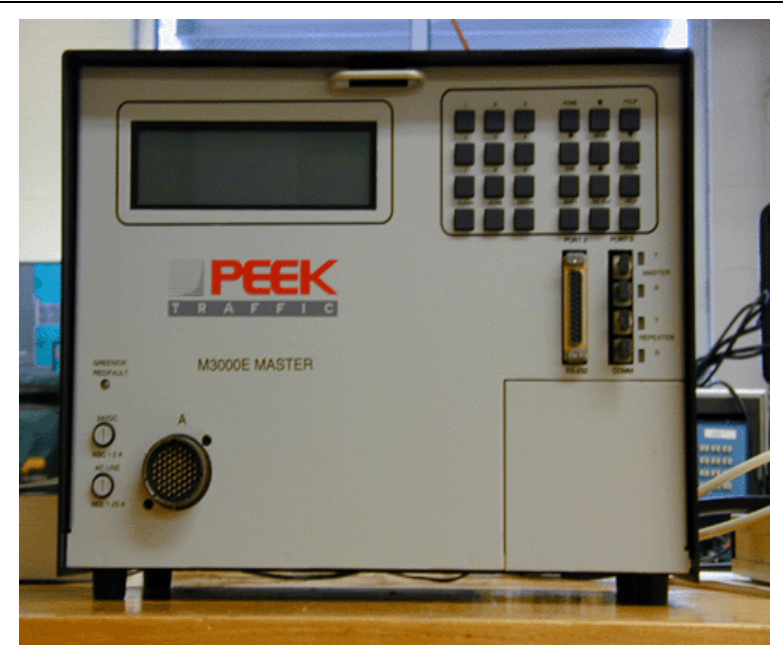

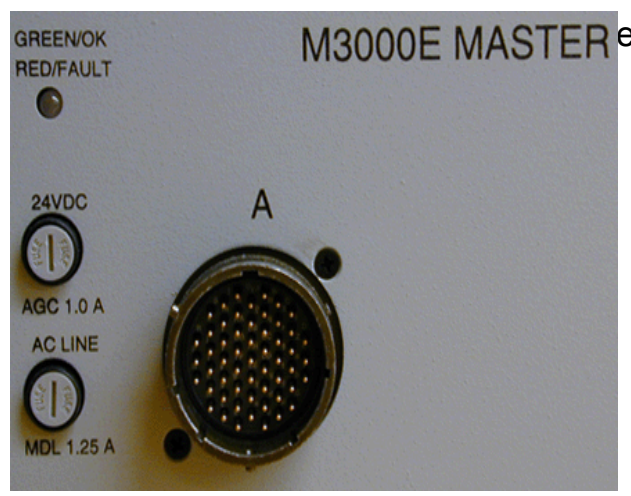

(b) NEMA A Connector

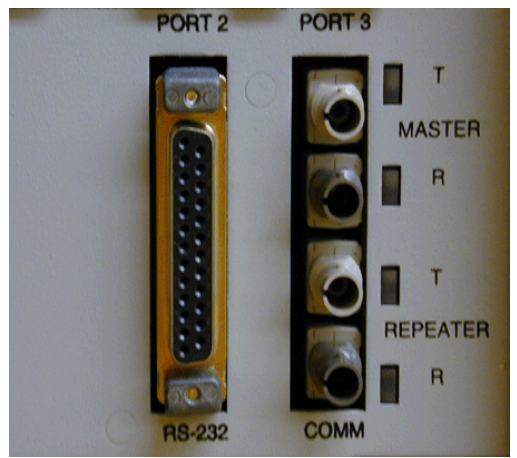

(c) Port 2 and Port 3

Figure 4-4 Master Controller and Connectors

The Peek M3000 master shown in Figure 4-4a has 1 connector and 2 ports on its front panel. The connector is used to interface with the signal cabinet. Port 2 is used when connecting directly to a computer or laptop or to exchange data over a telephone line with the CLMats software. When the master is interconnected with the local controllers, data can be exchanged with the local controllers through the master, requiring only one telephone line installation, in the cabinet with the master to communicate with an entire closed loop system. If data is exchanged by a direct laptop connection, this eliminates the need to go to each cabinet and directly connect to each local controller. Port 3 is the fiber port that is used to interconnect the master to the local

${ }^{20}$ Peek 3000 Operating Manual Rev 5.0, Appendix C. 
controllers. Specific pin assignments for the connector and ports can be found in the Peek Master Operating Manual. ${ }^{21}$

\subsubsection{Menus}

All menus in the controller can be accessed from the main menu. It is very important to become familiar with the keys and how the menus are set up. Data has to be entered in different formats, including numerical, yes/no, and enabled/disabled. It is very important to always press ENTER or cursor off of the last data entry before leaving a menu. The data will not be stored if a field is still active when the menu is exited. In the Peek controller main menu, there is an option to read data and an option to change data. The read data submenu only does not allow any data entry or modification and the change data submenu does allow it. The controller can be configured so that an access code is required to change data in the controller. However, anyone with access to the controller could look at the data under the read data submenu. Limited access will not be configured in the examples used in this report, so always use the change data submenu.
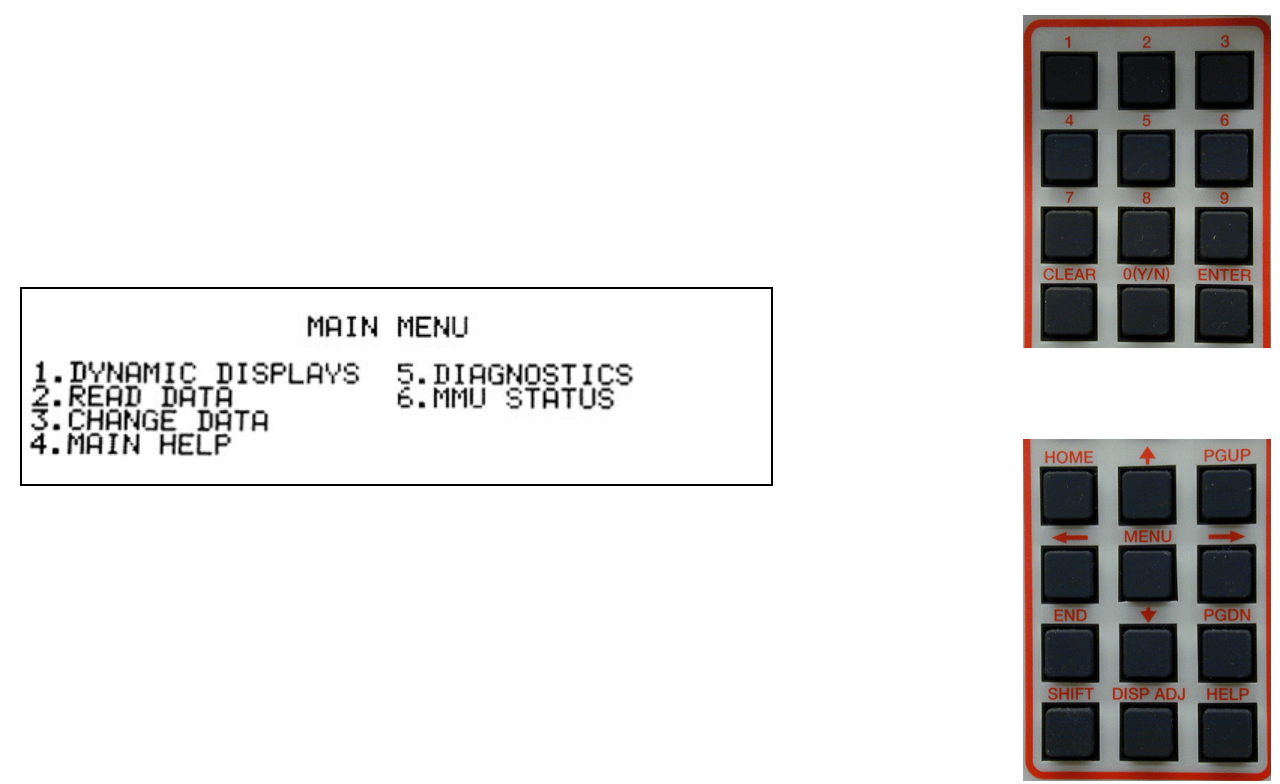

(a) Main Menu

(b) Keyboard

Figure 4-5 Peek Local Controller Main Menu and Keyboard

${ }^{21}$ Peek M3000 Master Operating Manual, Appendix B. 


\section{Home}

This key moves the cursor to the beginning of the line. Home + Shift moves the cursor to the first data field on the page.

\section{End}

This key moves the cursor to the end of the line. End + Shift moves the cursor to the last data field on the page.

\section{PgUp}

In multi-page data entry menus, this key displays the page before the current one displayed.

\section{PgDn}

In multi-page data entry menus, this key displays the page after the current one displayed.

\section{Arrow Keys $(\uparrow \rightarrow \downarrow \leftarrow)$}

These keys move the cursor in the corresponding direction. $\rightarrow+$ Shift will display menu screens to the right of the current one.

\section{Menu}

This key displays the previous menu screen. Menu + Shift displays the main menu.

\section{Disp Adj}

This key alone adjusts the contrast of the display screen by darkening it. Disp Adj + Shift lightens the screen.

\section{Help}

This key will display help screens related to the data being entered.

\section{Numbers (0-9)}

Press the number keys to access the corresponding menu and to enter data. Pressing shift and 0 will access menu 10.

\section{Toggle (0)}

Press 0 to toggle yes/no or to toggle through data fields that have more than one preset parameter.

\section{Enter}

Always press the Enter key after entering data before exiting the menu. Pressing Shift + Enter will open the Status Screen.

\section{Clear}

Press Clear to erase the current data entry and restore the previous data entry while the field is still active.

\section{Shift}

This key alone has no function. It is only used in conjunction with other keys as stated.

Figure 4-6 Peek Key Functions

The intersection status display shown in Figure 4-7 provides real-time information about the state of the controller:

1. In regular operation, the top line shows the cycle/offset/split that is active and the cycle timer. If the unit is not in coordination, "Free" is displayed. The top line of the screen is also used for special messages if there are flash conditions or errors. More information about these errors can be found in the Peek Operating Manual. ${ }^{22}$

2. The numbers 1 through 16 at the top of the screen represent phases 1 through 16 .

3. The $V$ Demand row shows vehicle calls on each phase. A ' $V$ ' indicates a vehicle detector call, an ' $M$ ' indicates the vehicle call is locked in memory if this feature is enabled, an ' $r$ '

\footnotetext{
${ }^{22}$ Peek 3000 Operating Manual Rev 5.0, Dynamic Displays.
} 
indicates the phase is in minimum recall, and an ' $R$ ' indicates the phase is in maximum recall.

4. The $P$ Demand row shows the pedestrian calls on each phase. A ' $P$ ' indicates a pedestrian detector call and an 'M' indicates the pedestrian call is locked in memory, if this feature is enabled.

5. The bottom of the screen shows which phases are active (ON) in each ring, along with which phases will be served next (NX).

6. The timing intervals for the phases that are active are shown, along with their timers. Some of the common intervals shown are initial, or minimum green (INIT), green rest (GRST), vehicle extension (PASS), yellow clearance (YEL), and red clearance (RED).

7. The max timers and termination codes for the active phases are shown. The common termination codes shown are gap out (GAP TERM), max out (MAX TERM), and coordination force off (FORCE TERM).

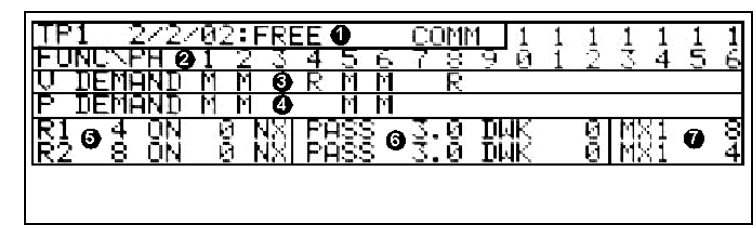

Figure 4-7 Controller Status Screen (Shift + Enter)

\subsubsection{Memory}

The Peek controller has two areas of memory - the RAM and the EEPROM. The RAM stores the current information being used by the controller, while the EEPROM (Figure 4-8) serves as the permanent backup. When data is entered with the keypad, the information is stored in the RAM. This data can be copied to the EEPROM and the EEPROM removed from the controller once the programming is complete. The EEPROM can be placed on the shelf next to the controller in the cabinet. In case of power failure or some other event that could erase the memory in the controller, the EEPROM can be placed in the controller and the configuration restored immediately. Figure 4-9a shows the menu used to copy to and from the EEPROM. Figure 4-9b shows the menu to enabled data to be copied directly to the EEPROM when it is entered by the keypad. These options are available for the local and master controllers. 


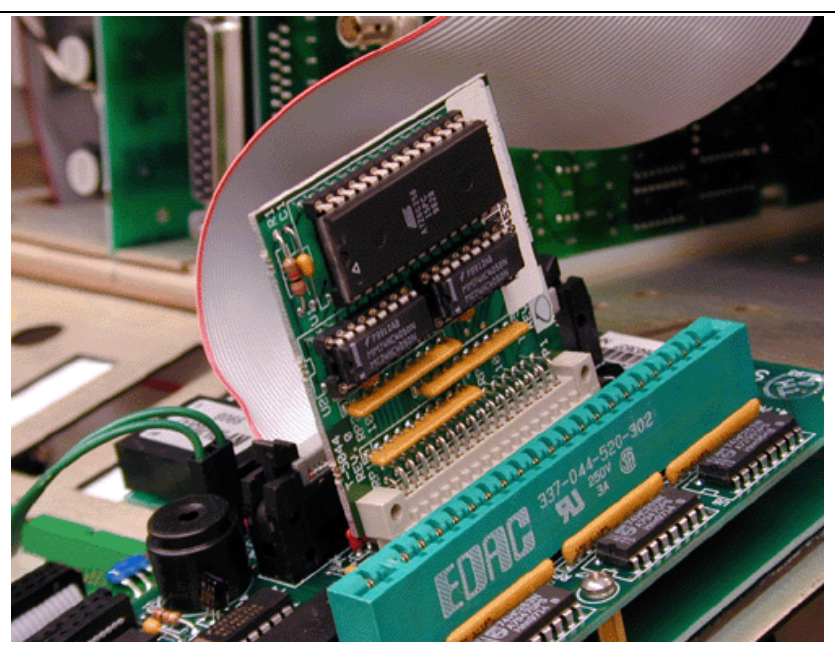

Figure 4-8 Controller EEPROM

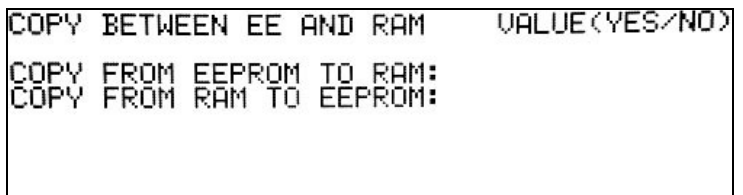

(a) RAM \& EEPROM Copy (MM, 3, 6, 4)

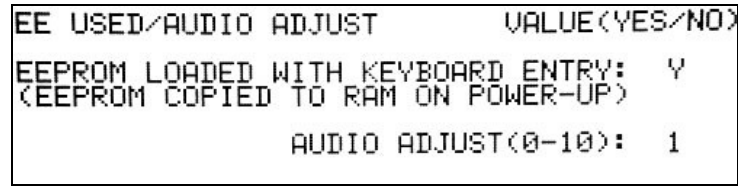

(b) Copied to EEPROM (MM, 3, 5, 3)

Figure 4-9 Controller Memory Screens 


\subsection{Initializing the Controller}

Prior to programming the controller, it is very important to erase unwanted information that may exist in the controller. Not clearing the memory can lead to many problems in the future. For example, irrelevant time-of-day programs could be entered or unused detectors could be enabled. Troubleshooting will also be simplified by initializing the controller.

\subsubsection{Local Procedure:}

Main Menu $\rightarrow$ 3. Change Data $\rightarrow 6$. Utilities $\rightarrow$ 1. Default Data Load

The quickest way to clear the memory and start with a valid timing plan is to load default data. In the Peek controller, data in five different submenus in the change data menu can be loaded.

These submenus include:

- Submenu 1 Controller

- Submenu 2 Coordination

- Submenu 3 Time-of-day

- Submenu 4 Preemption

- Submenu 5-4 Special-Options.

In this menu, selecting option 6 will load default data into all of the above submenus. Note that not all data in the controller can be cleared. Data in the submenus not listed might be a starting point when troubleshooting the controller.

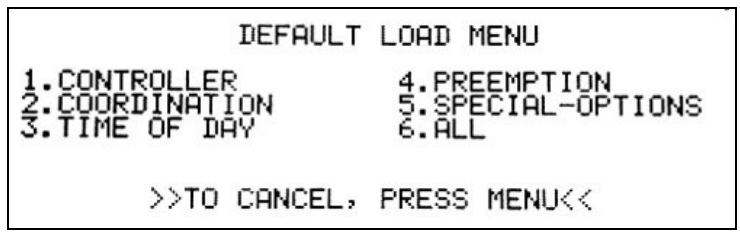

(a) Load Default Data (MM, 3, 6, 1)

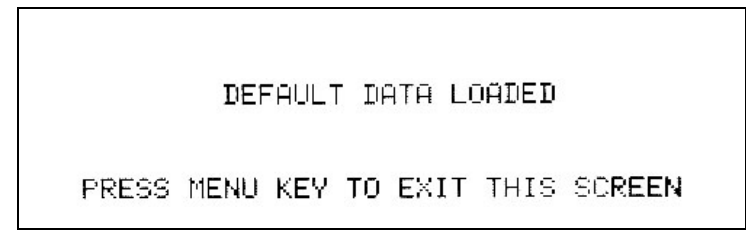

(b) Default Data Loaded

Figure 4-10 Initialization: Local Controller Default Data Load 


\subsubsection{Master Procedure:}

Main Menu $\rightarrow$ 3. Change Data $\rightarrow$ 7. Utilities $\rightarrow$ 1. Load Default Data

In the Peek master controller, default data can be loaded for each of the following submenus under change data:

- Control - master control, local control, sensors

- Channels - computational channel menu

- Time of Day - time of day menu

- Pattern - pattern menu

- Communications - setup and printer functions

- Options - is not used at this time.

By choosing option 7 in this menu, the default data will be loaded for all of these submenus.

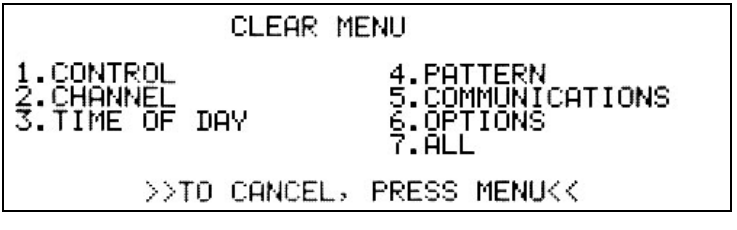

(a) Load Default Data (MM, 3, 7, 1)

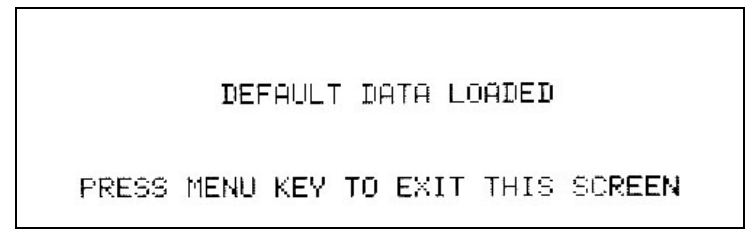

(b) Default Data Loaded

Figure 4-11 Initialization: Master Controller Default Data Load 


\subsection{Setting up Communication}

\subsubsection{Interconnected System with Master}

Once the master and local controllers are initialized, communication between them should be established. The Peek master can control and monitor up to 4 closed loop zones. It can call different plans for each zone and collect data from each zone. Before proceeding, make sure all local controllers are connected to the master with the appropriate telemetry cable. The Peek 3000 series controllers can be interconnected using a fiber optic line. The physical fiber connection of a three-intersection closed loop system is shown in Figure 4-12. Fiber optic setup will be described here.

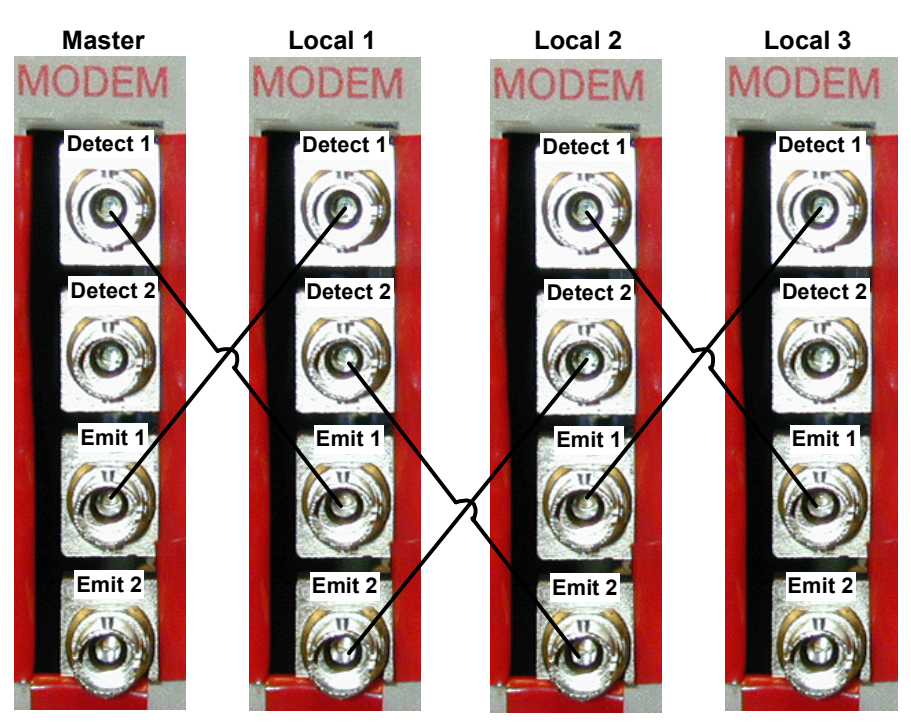

Figure 4-12 Fiber Optic Setup

\subsubsection{Telemetry Assignments}

\subsection{Local Procedure:}

Main Menu $\rightarrow$ 3. Change Data $\rightarrow 5$. Special $\rightarrow 5$. Closed Loop: Intersection/Zone Identification In the intersection/zone identification menu, the controller is assigned to a master and assigned an identification number. In the intersection identification menu shown in Figure 4-13, the master type is configured. Zero should be also entered here if the controller is at an isolated intersection. The 3000 series local controller is compatible with the 3000 series master and the 3800EL master. In this example, the 3000 series master is used. Each local controller shall be assigned 
a unique identification number from 0-255. Zero should only be used if the intersection is an isolated intersection, not running in a closed loop system. In this example, the intersections are number 1-5, with the example intersection assigned as 1 . Next, the master identification number is entered. In this example it is set to 1 . The master identification number will be configured in the master controller later.

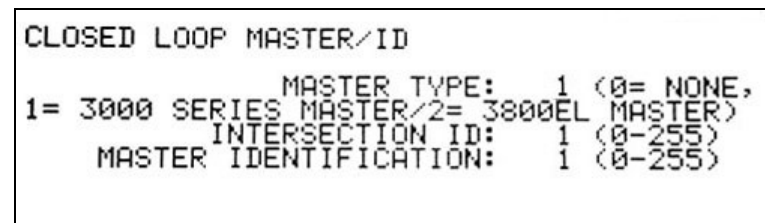

Figure 4-13 Communication: Local Controller Identification (MM, 3, 5, 5)

Main Menu $\rightarrow$ 3. Change Data $\rightarrow$ 5. Special $\rightarrow$ 4. Special-Options $\rightarrow$ 3. Comm Set-up

In the first comm set-up screen shown in Figure 4-14, the port that is used to connect to the master is entered. In this example, port 3 , the fiber optic interconnect will be used. The monitor and central ports are not used here.

\begin{tabular}{|c|c|}
\hline COMM SET-UP & $(G=$ NONE, $2-3=$ PORT $2-3\rangle$ \\
\hline MASTER(LL) & FORT: 3 \\
\hline MONITOR & PORT: \\
\hline $\begin{array}{l}\text { CENTREL } \\
\text { FGIN FIDR }\end{array}$ & 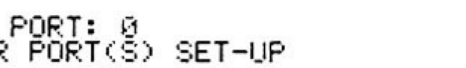 \\
\hline
\end{tabular}

Figure 4-14 Communication: Local Controller Master Port Configuration (MM, 3, 5, 4, 3)

Direct connect communication with a computer or laptop occurs through port 2. The port 2 setup screen shown in Figure 4-15 is found in the second screen of the comm set-up menu. The baud rate can be set to $1200,2400,4800,9600,14400$, or 19200 bps. A recommended starting point is 9600 bps. If port 2 communication is not working, the baud rate might have to be changed. For use with CLMats, the parity is set to even and the data bits are 8 . 


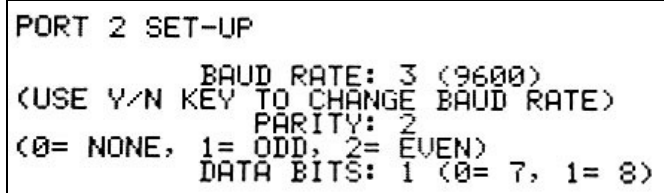

Figure 4-15 Communication: Local Controller Port 2 Configuration (MM, 3, 5, 4, 3, PgDn)

In this situation, port 3 is used for the fiber optic interconnect. The port 3 configuration menu shown in Figure 4-16 is located in the third screen of the comm set-up menu. The same options are available for port 3 as port 2 . Here the baud rate will again be set to 9600 bps. The parity will be even and data bits 8 .

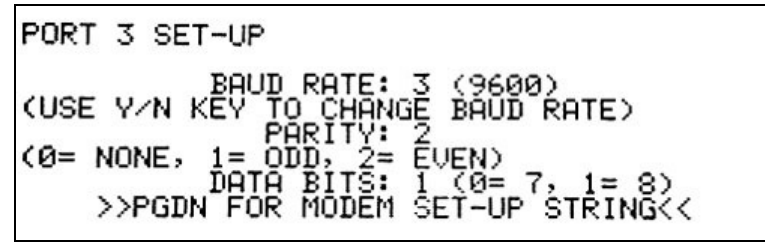

Figure 4-16 Communication: Local Controller Port 3 Configuration (MM, 3, 5, 4, 3, PgDn(2))

\subsection{Master Procedure:}

Main Menu $\rightarrow$ 3. Change Data $\rightarrow$ 1. Master Control

In the master control menu shown in Figure 4-17, the master identification number is set. This number shall be consistent with the one entered in the local controllers. Here, the master identification is 1 . Other parameters in this menu will not be configured at this point.

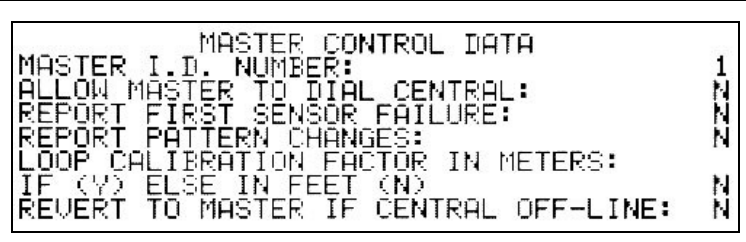

Figure 4-17 Communication: Master Controller Identification Configuration (MM, 3, 1) 
Main Menu $\rightarrow$ 3. Change Data $\rightarrow$ 8. Special $\rightarrow$ 3. Comm Set-up

As with the local controller, direct connect communication with a computer or laptop occurs through port 2. The port 2 setup screen shown in Figure 4-18 is found in the first screen of the comm set-up menu. The baud rate will also be set to $9600 \mathrm{bps}$, the parity is set to even, and the data bits are 8 .

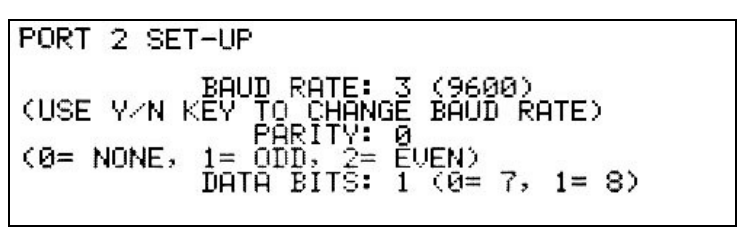

Figure 4-18 Communication: Master Controller Port 2 Configuration (MM, 3, 8, 3)

Again, port 3 is used for the fiber optic interconnect. The port 3 configuration menu shown in Figure 4-19 is located in the second screen of the comm set-up menu. The baud rate will again be set to 9600 bps. The parity will be even and data bits 8 .

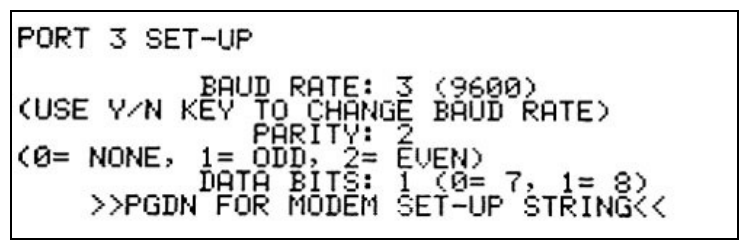

Figure 4-19 Communication: Master Controller Port 3 Configuration (MM, 3, 8, 3, PgDn)

Main Menu $\rightarrow$ 3. Change Data $\rightarrow$ 2. Master to Local

In the master to local menu shown in Figure 4-20, each local controller should be activated by changing the ' $N$ ' to ' $Y$ '. The zone number used here will be 1 . All five intersections will be in the same zone since they constitute one system.

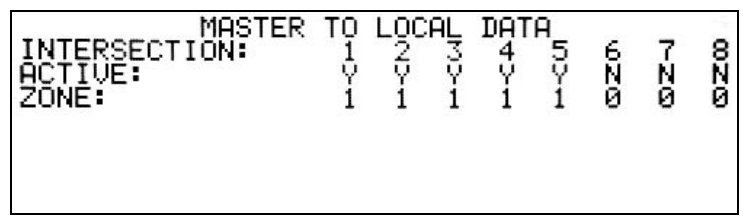

Figure 4-20 Communication: Master Local Enable (MM, 3, 2) 


\subsubsection{Telemetry Verification}

\subsection{Master Procedure:}

Main Menu $\rightarrow$ 1. Dynamic Displays $\rightarrow 1$. Sys Operation Menu $\rightarrow 2$. Intersections

The primary method of verifying communication is to check the intersection display shown in Figure 4-21 in the master. Under each intersection number, an ' $A$ ' indicates that communication between the master and that local is active, an ' $\mathrm{M}$ ' indicates the communication is marginal which usually means data is only traveling in one direction, and ' $L$ ' indicates that there is no communication occurring. Having marginal communication is sometimes a result of one of the fiber optic connectors plugged into the wrong port. If there is no communication at all, make sure the data rates, parity, and bits are correct and the intersection identification is correct.

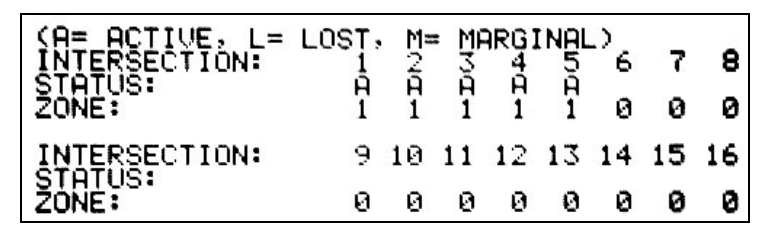

(a) Telemetry Active

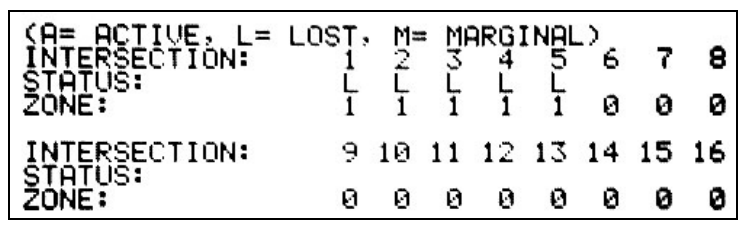

(b) Telemetry Lost

Figure 4-21 Communication: Master Telemetry Verification (MM, 1, 1, 2)

\subsubsection{Interconnected System Time Clock}

\subsection{Master Procedure:}

Main Menu $\rightarrow$ 3. Change Data $\rightarrow 3$. Time of Day $\rightarrow$ 8. Set Date and Time

One of the many advantages of interconnected systems is clock synchronization. The master can set all local controller time clocks. This is very important for coordinated systems. Without the same time references, the controller offsets are useless. To set the clock, go to the time of day menu screen shown in Figure 4-22. Enter must be pressed in order for the changes to take effect. This time will be downloaded to all local controllers. Also, the master will regularly synchronize the time of all local controllers. 


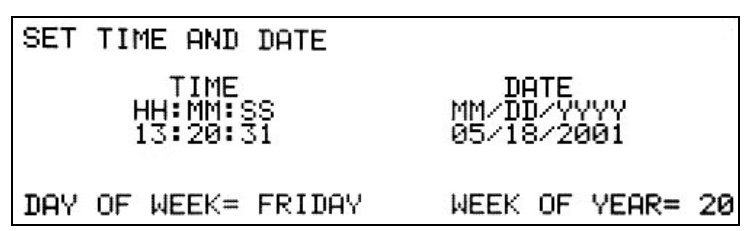

Figure 4-22 Master Time and Date Configuration (MM, 3, 3, 8)

\subsubsection{Non-interconnected System}

In general, an interconnected system is preferred because it is easier to ensure synchronization. However, situations arise where it is not practical to deploy communication infrastructure to interconnect traffic signal equipment. In those cases, it is critical that all controllers be configured to reference the identical time. When programming the time clocks, extra care must be taken to program the clocks to the nearest second. A device such as a handheld Global Positioning System (GPS) receiver is usually the most precise and accurate time reference to use for this procedure.

\subsubsection{Local Procedure:}

Main Menu $\rightarrow$ 3. Change Data $\rightarrow 3$. Time of Day $\rightarrow$. Set Date and Time

If a system with multiple controllers is running coordination plans, the time clock in each controller needs to be set. The clock in each controller needs to be as accurate as possible for optimal performance. Time clocks in the controllers will actually drift over time. Controllers in the field should be reset every year or after power outages to ensure synchronization. To set the time clock, go to the time of day menu screen shown in Figure 4-23. Enter must be pressed for the changes to take effect.

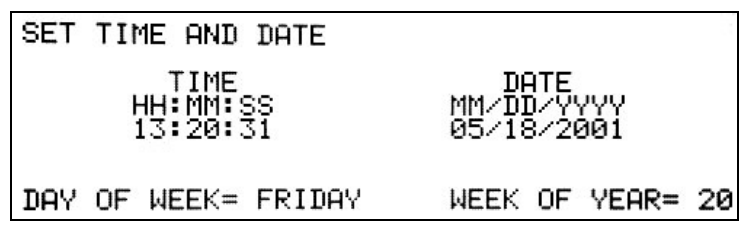

Figure 4-23 Local Controller Time and Date Configuration (MM, 3, 3, 9) 


\subsection{Closed Loop MATS: Computer Interface}

Once the communication has been established, assuming all controllers are linked via telemetry, configuration of the system can be performed with CLMats management software. The CLMats software has many benefits besides simplifying controller data entry. The typical sequence followed when using CLMats is to upload the current controller database to the computer, modify it, and download the new database back to the controller. Before the data is downloaded to the controller, it is saved on the computer. This backup copy is always available in case something happens to the controller and the data is erased. Instead of keying in all of the data by hand, which could take hours, the data is simply downloaded and the intersection is running again in a few minutes. Furthermore, with the modem plugged into a phone line, new timing plans can be entered into CLMats and downloaded to the controllers without leaving the office, although this practice is discouraged for safety reasons. Other CLMats features include green band analysis for showing coordination and real-time intersection displays that allow the user to assign phases and detectors to images.

Example screens in this section are from Intersection 1 State Street in the U.S. 231 system. Data for this system can be found in Appendix B. For each parameter configured, the CLMats screen will be displayed with the corresponding controller screen and the Synchro screen from which the data was taken.

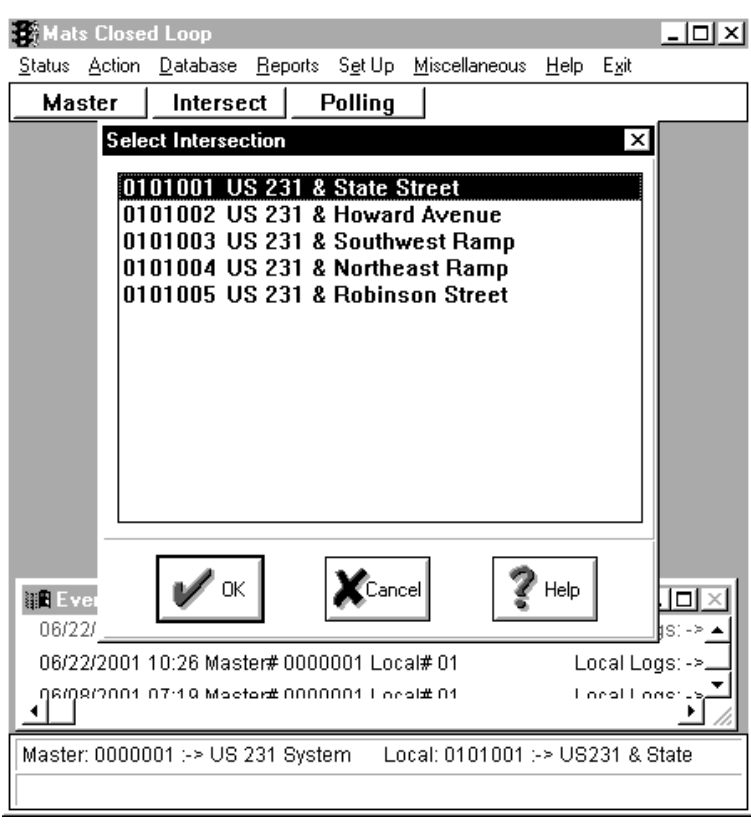

Figure 4-24 CLMats Computer Software 


\subsubsection{Preparing to Upload}

Although all submenus in the "Change Data" menu can be modified in CLMats, it is highly recommended that certain parameters be configured before the database is uploaded so a common procedure can be used for all closed loop systems in Indiana. These parameters include phase sequences, overlaps, and preemption.

\subsubsection{Controller Configuration}

Main Menu $\rightarrow$ 3. Change Data $\rightarrow 1$. Controller $\rightarrow 1$. Sequence Configuration

The controller sequence is used to configure the ring structure for the intersection. The ring structure for this intersection is shown in Figure 4-25a. In the first screen in the sequence configuration, default ring structures can be selected. The 8 phase quad left configuration corresponds to the NEMA ring structure and is used here. By selecting this choice in Figure $4-25 \mathrm{~b}$, the screen in Figure 4-25d will automatically be programmed. Ring 1 has vehicle phases 1-4 and pedestrian phases 2 and 4. Ring 2 has vehicle phases 5-8 and pedestrian phases 6 and 8. The barriers are located between phases $2 / 6$ and $3 / 7,4 / 8$ and $1 / 5$. In the Peek controller, phases on a common side of a barrier are in the same co-phase. Here, phases 1, 2, 5, and 6 are in co-phase 1 and phases $3,4,7$, and 8 are in co-phase 2 . The pedestrian and overlap phases are not exclusively timed phases, so they are not included in the ring structure. 


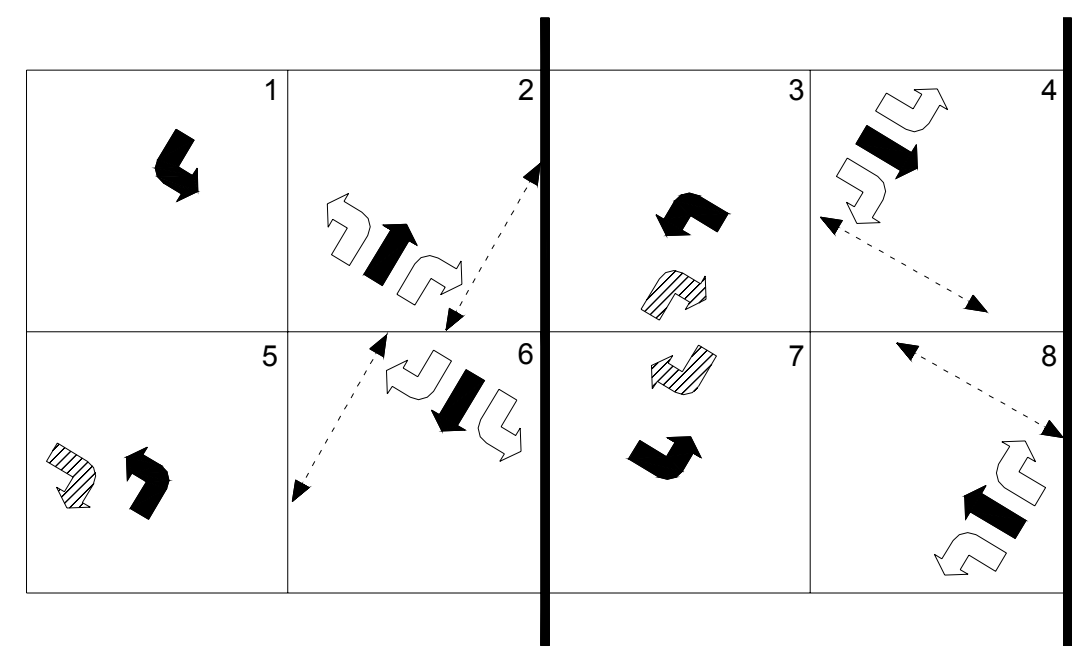

(a) Ring Structure
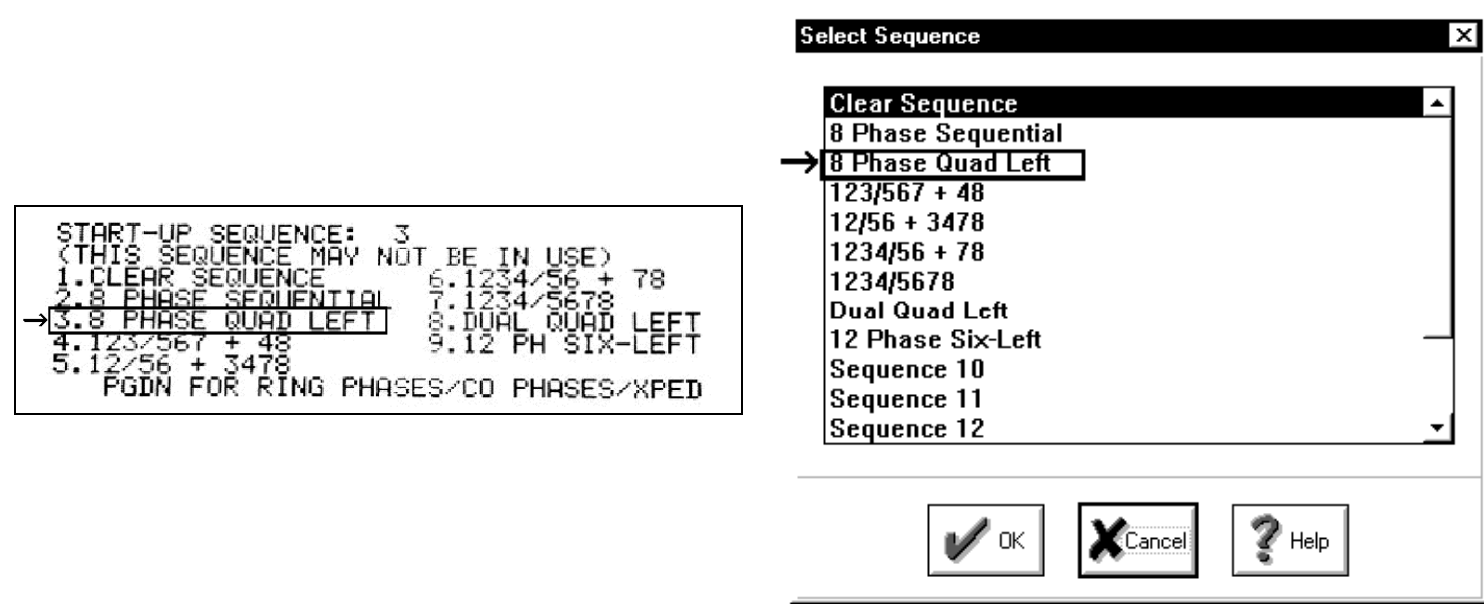

(b) Controller (MM, 3, 1, 1)

(c) CLMats (after upload)

\begin{tabular}{|c|c|}
\hline $\begin{array}{l}\text { UALUEY YES } \\
\text { FUNCPH } \\
\text { RING } 1 \\
\text { RING } 2 \\
\text { RING } 3 \\
\text { RING } 4\end{array}$ & 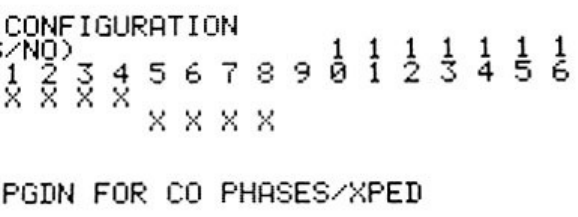 \\
\hline $\begin{array}{l}\text { RHA } \\
\mathrm{RINGS} \\
\mathrm{PH} \\
\mathrm{PH} \\
\mathrm{PH}\end{array}$ & 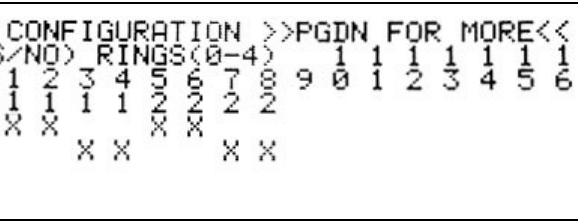 \\
\hline
\end{tabular}

(d) Controller (MM, 3, 1, 1, PgDn)

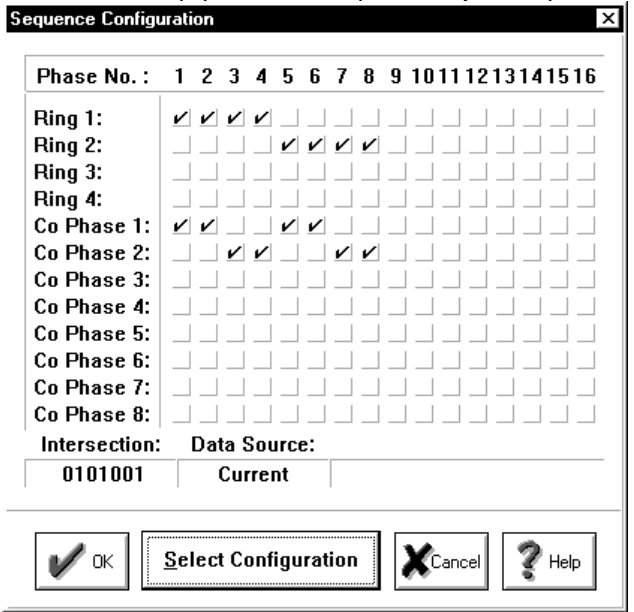

(e) CLMats (after upload)

Figure 4-25 Controller Sequence Configuration 


\subsubsection{Overlap Assignment}

Main Menu $\rightarrow$ 3. Change Data $\rightarrow$ 1. Controller $\rightarrow 6$. Overlaps $\rightarrow 1$. Standard Overlaps

Intersection 1 in this system has right-turn overlaps on three approaches. These overlaps will run concurrently with the appropriate left-turn phases as shown in Figure 4-25a. Overlap A, which is the phase 2 northbound right-turn movement, will run concurrently with phase 3 , the westbound left-turn movement. In the screen shown in Figure 4-26a, the parent phase of overlap A should be phase 3. Similarly, overlap B has parent phase 5 and overlap $C$ has parent phase 7. 
OUERLAP $\mathrm{A}(1 \mathrm{OF} 16) \quad F L A S H$ CODE $(0-7): 0$ MODE (Q GTD, 2=NDT FED, $3-6=$ TURN HD':

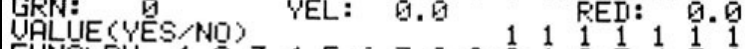

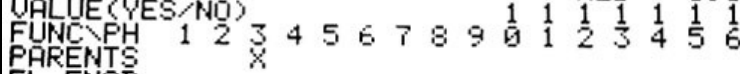

FL ENAB

(a) Controller Overlap A (MM, 3, 1, 6, 1)

Phase Programming:

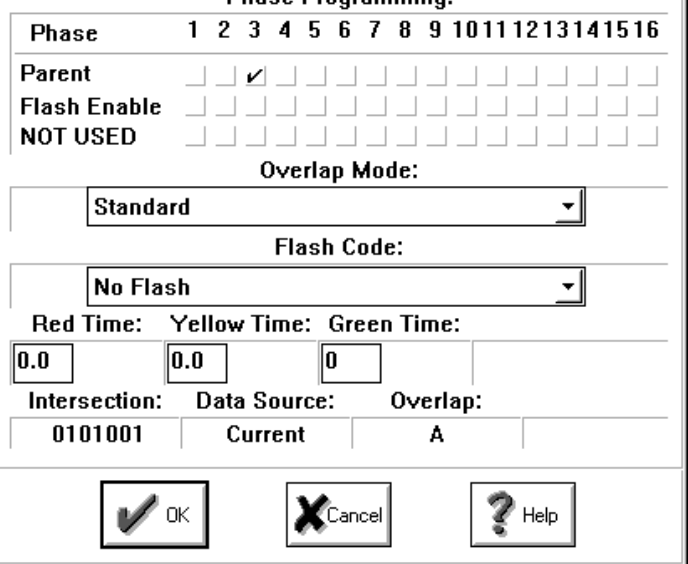

(b) CLMats Overlap A (after upload) Standard Overlap

Phase Programming:

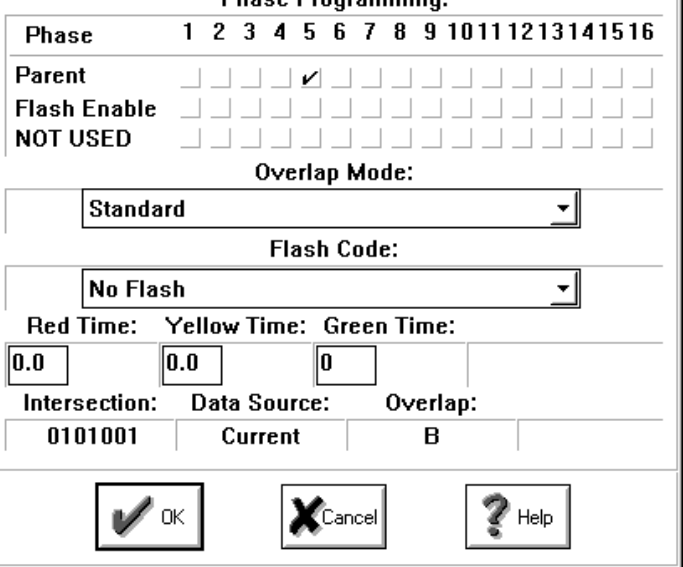

(c) Controller Overlap B (MM, 3, 1, 6, 1, PgDn)

(d) CLMats Overlap B (after upload)

Standard Overlap

OUERLAP E $(2$ OF 16 ) FLASH CODE (0-7):

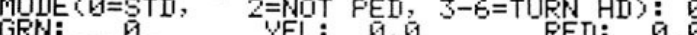

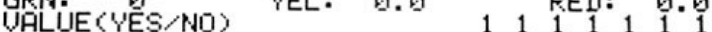
FUNC PH $123456789012 \frac{1}{3} 456$ FL ENAB

QUERLAP $C$ ( 3 OF 16 ) FLPSH COIE (0-7): MONE (Q $=S T D, \quad 2=N O T$ FEI, $3-6=T U R N$ HD): E. REII G.

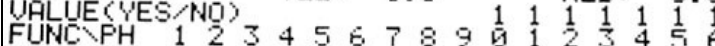
PARENTS FL ENAB

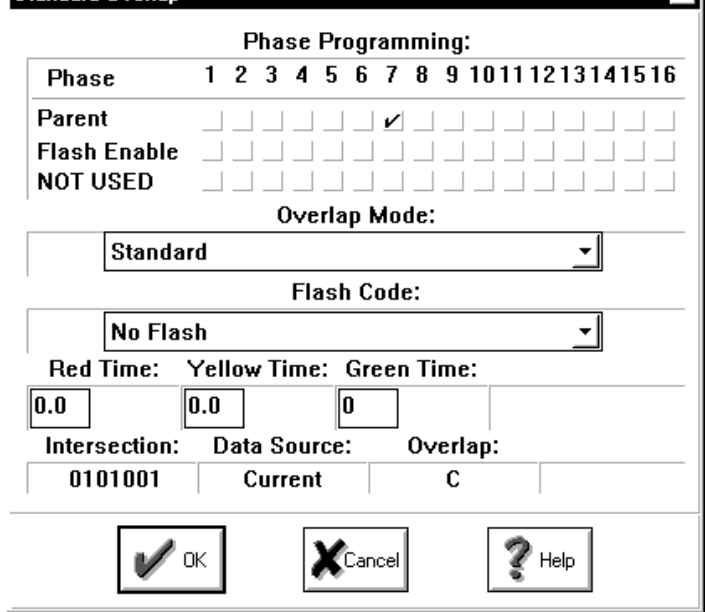

(e) Controller Overlap C (MM, 3, 1, 6, 1, PgDn (2))

(f) CLMats Overlap C (after upload)

Figure 4-26 Phase Overlap Assignment 


\subsubsection{Preemption}

Main Menu $\rightarrow$ 3. Change Data $\rightarrow$ 4. Preemption

If preemption is used at this intersection, it can be entered here. There are six preemptor sequences available in the controller. Each sequence can be programmed to serve the desired movements. Sample screens for priority preemptor 1 are shown in Figure 4-27. Since preemption is not commonly used in Indiana, it will not be discussed further. More information on configuration can be found in the Peek operating manual. ${ }^{23}$

${ }^{23}$ Peek 3000 Operating Manual Rev 5.0, Preemption. 


\subsubsection{Setting up a CLMats System}

\subsubsection{CLMats Procedure:}

Set Up $\rightarrow$ Define Master $\rightarrow$ Edit

A new master must be defined in CLMats before data can be uploaded. After CLMats has been launched on the computer, go to set up and define master. For this example, the master is number 1. Highlight No. 1 and press the edit button as shown in Figure 4-28a. If master number 1 is already used, select an available master number. This new master \# will have to be entered into the master and local controllers using the procedure documented in Section 4.3. Figure $4-28 \mathrm{~b}$ shows the screen to edit the master information. The master type is entered as $3800 \mathrm{EL}$ or M3000. The connection channel will vary based on which communication port the modem is installed on or which communication port is used for direct connection. The actual phone number to be dialed should be entered here. The master number on this screen is actually the master id. This number does not have to correspond to the master id number discussed earlier. This id is only used as a reference. The master name and short name should be entered to help identify the system. Notice that isolated local intersections can also be managed with CLMats by selecting that option in this screen (Figure 4-28).

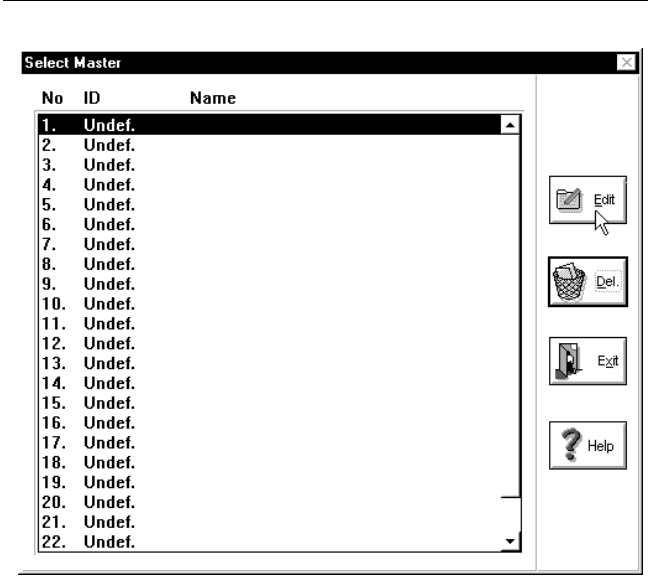

(a) Select Master

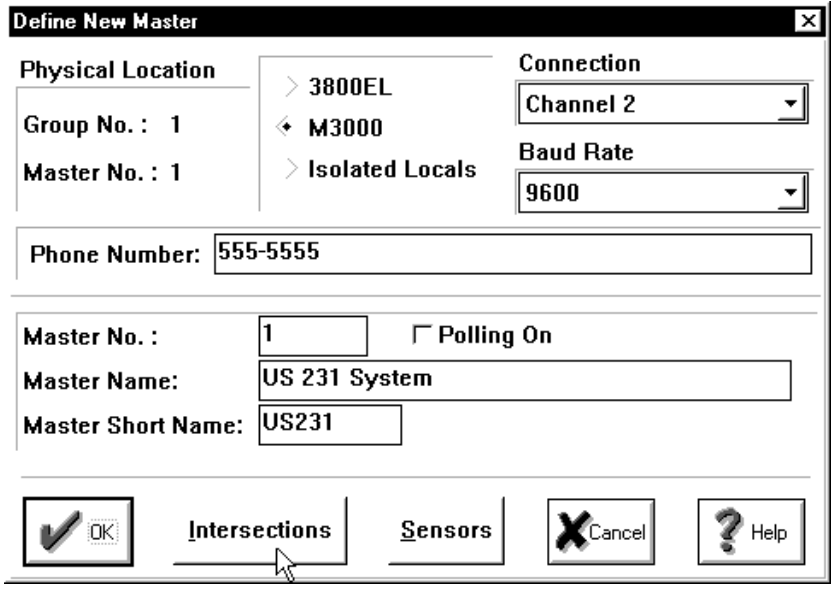

(b) Edit Master Information

Figure 4-28 CLMats: Define Master

Set Up $\rightarrow$ Define Master $\rightarrow$ Edit Master Information $\rightarrow$ Intersections

Intersections are added to each master within its edit screen shown in Figure 4-28b by clicking on the intersections button. 
1. The intersection numbers should match the identification numbers entered in the controllers. Here, intersection numbers 1-5 are used. Highlight the corresponding intersection number and press the edit button as shown in Figure 4-29a. The intersection information can be edited in the screen shown in Figure 4-29b.

2. The seven digit intersection number here is an identification number used only in the software. It does not have to correspond to the controller identification number. The numbering convention that is typically used is the first two digits are the zone number, the second two digits are the master number, and the last three digits are the intersection number. Here the identification number is 0101001 for zone 1 , master 1 , and intersection 1.

3. The local and regional names are entered to help identify the location. The main street is the name of the arterial of the system. The cross street is the name of the street crossing the main street at this intersection. The short names are six character names, usually abbreviations.

4. The through phase assignments are entered here for the main and cross streets. Using the NEMA numbering scheme, the arterial phases should be 2 and 6 , the cross street phases should be 4 and 8 .

5. The local controller type at this intersection is selected.

All intersections in the system should be entered before proceeding.

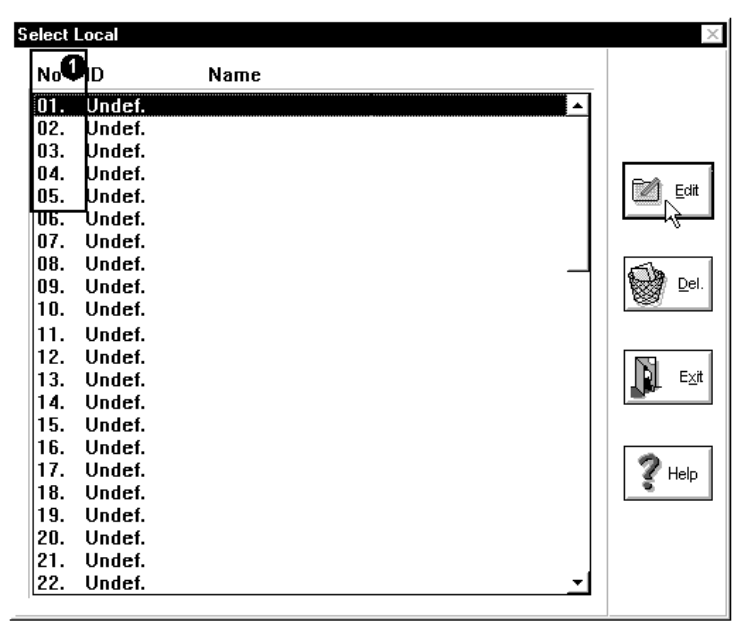

(a) Select Intersection

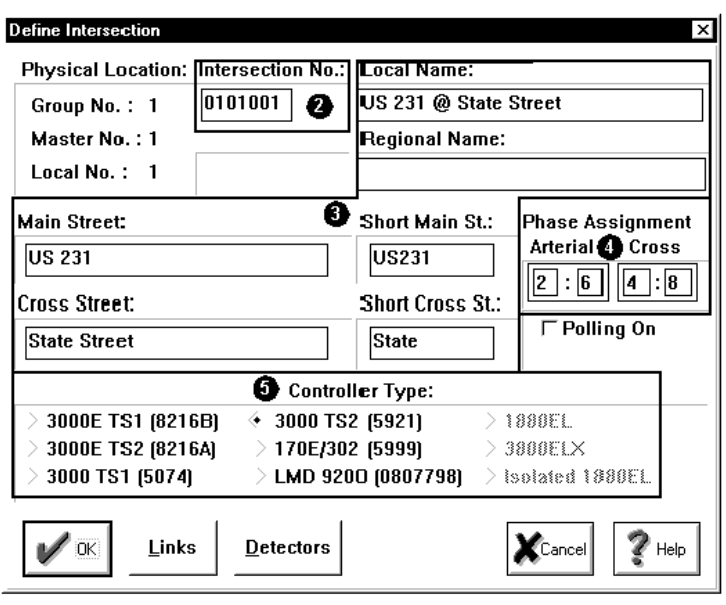

(b) Edit Intersection Information

Figure 4-29 CLMats: Define Intersections 


\subsubsection{Uploading to CLMats}

The controller databases are now ready to be uploaded to CLMats. In order to communicate with a particular master and local, they have to be active in the bottom of the CLMats window. To choose the active master, click on the Master button at the top of the CLMats window in Figure 4-30. Select the desired master. To choose an intersection, click on the Intersect button at the top of the CLMats window. The intersections can be sorted by system number, full main street name, or short main street name. This is where consistent intersection identification numbering is helpful. Select the desired intersection. Make sure that the intersection selected is in the same system as the master selected. The master and local selected should be displayed at the bottom of the window, as shown in Figure 4-30.

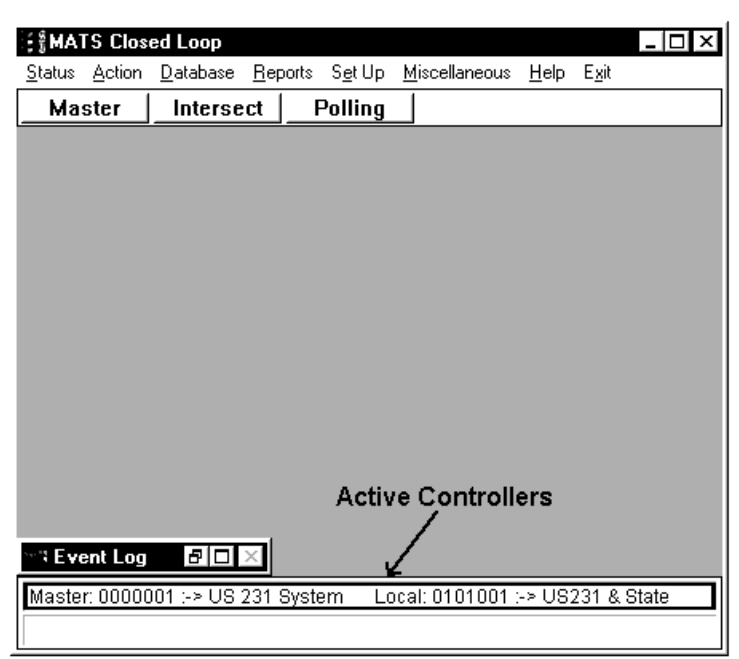

Figure 4-30 CLMats: Activate Master and Local

\subsubsection{Setting up Communication for CLMats}

\section{Set Up $\rightarrow$ Configure Channels $\rightarrow$ Configure Communications}

The computer port settings need to be configured before communicating with the controller. These settings will be specific to the computer being used. Recommended settings are given in Table 4-1. If CLMats sends a message and either receives no answer or a corrupted reply, then the quantity of retries is how many times CLMats will rebroadcast that message until it lists a communications failure. If CLMats sends a message and either receives no answer or a corrupted reply, then the amount of time in milliseconds between retries is the Time Out value. Tweak is the amount of time in milliseconds required for character definition and is proportional to the baud rate. After the settings are configured, click on the button "Use New Settings Now" 
shown in Figure 4-31a. These settings can also be configured in the communications server shown in Figure 4-31d. This window automatically opens when CLMats opens. While data is being uploaded and downloaded, the progress of the transmission can also be viewed here.

Table 4-1 Communication Settings

\begin{tabular}{|c|c|c|}
\hline Controller & M3000/3000 & $\mathbf{3 8 0 0 E L / 1 8 8 0 E L}$ \\
\hline Baud Rate & $<19,200$ & 1,200 \\
\hline Bits & 8 & 7 \\
\hline Stop Bits & 1 & 1 \\
\hline Parity & None & Even \\
\hline Retries & \multicolumn{2}{|c|}{5000} \\
\hline Timeout & \multicolumn{2}{|c|}{ 6 for a baud of 1,200} \\
\hline Tweak & 32 for a baud of 9,600 \\
& 64 for a baud of 19,200 \\
\hline
\end{tabular}




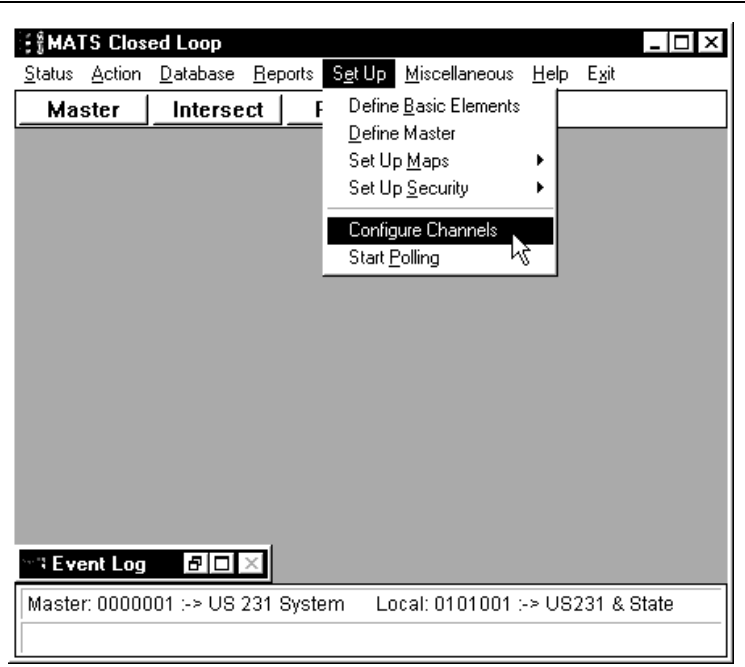

(a) Configure Menu

\begin{tabular}{|c|c|c|c|}
\hline \multicolumn{3}{|c|}{ Channel Setup } & $\bar{x}$ \\
\hline \multicolumn{3}{|c|}{ Channel Number $1 \exists$} & Exit \\
\hline \multicolumn{3}{|c|}{ Port Connection } & SAVE \\
\hline \multicolumn{2}{|l|}{ Baud Rate- } & & \\
\hline \multirow{2}{*}{$C_{19200}$} & $C_{4800}$ & c 9600 & C 14400 \\
\hline & $C 28800$ & C 38400 & $C_{56 \mathrm{~KB}}$ \\
\hline 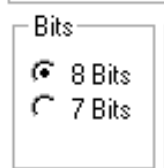 & $\begin{array}{l}\text { Stop Bits- } \\
\text { C } 1 \text { Stop } \\
C 2 \text { Stop }\end{array}$ & $\begin{array}{l}\text { Parity- } \\
\odot \text { NONE } \\
r \text { EVEN } \\
r\end{array}$ & $\begin{array}{c}C \text { MARK } \\
C \text { SPACE } \\
\text { DD }\end{array}$ \\
\hline \multirow{2}{*}{$\begin{array}{l}\text { Retrys } \\
5\end{array}$} & Out & & \\
\hline & & $\Gamma \mathrm{D}$ & able this Channel \\
\hline
\end{tabular}

(c) Settings Screen

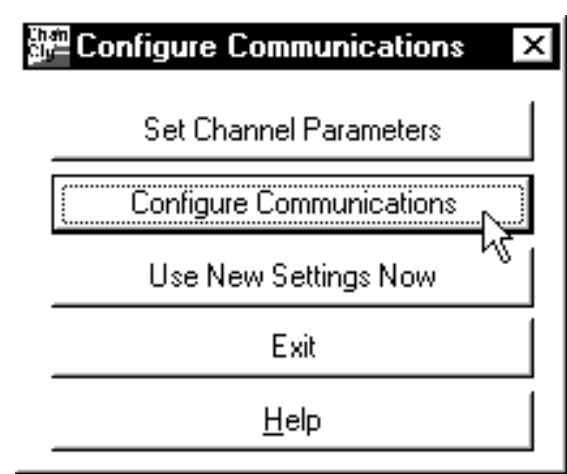

(b) Communication Settings

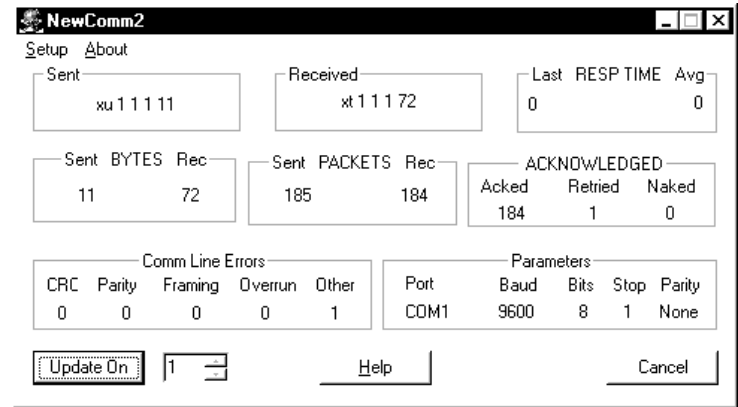

Figure 4-31 CLMats: Configure Communications

\section{Status $\rightarrow$ Intersection Status $\rightarrow$ Controller Front Panel}

The quickest way to determine if communication is active is to look at the local controller front panel via CLMats. This will connect to the active local controller and show the same display that is on the actual controller. If the display does not show up as it is shown in Figure 4-32, communication is not established and the communication settings need to be adjusted. 


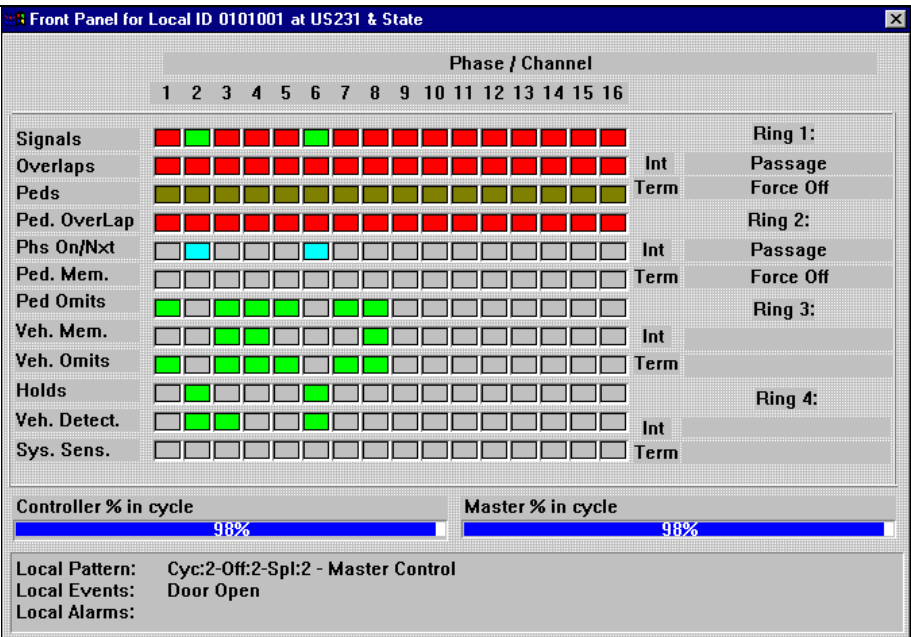

Figure 4-32 CLMats: Controller Front Panel

\subsubsection{Master Upload}

Action $\rightarrow$ Master $\rightarrow$ Upload/Download Settings

In the master, the same data can be modified in CLMats that was entered by the default data load discussed in Section 4.2. The data that is uploaded can be selected in the upload/download options menu shown in Figure 4-33.

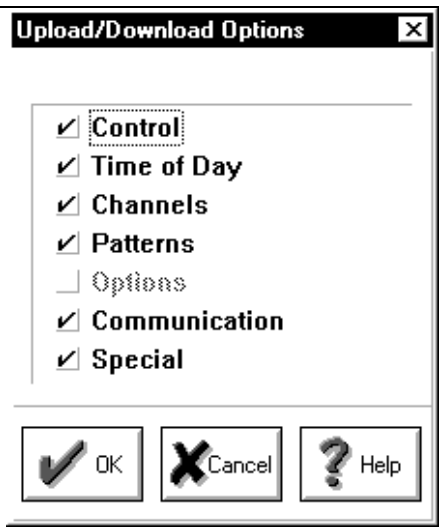

Figure 4-33 CLMats: Master Upload/Download Settings

Action $\rightarrow$ Master $\rightarrow$ Upload

The master database can now be uploaded. If an error occurs during the upload, check the communication settings. After the master database has been uploaded, it can be compared to 
the existing database in the computer. At this point, the database in the computer is not a valid one and should be overwritten.

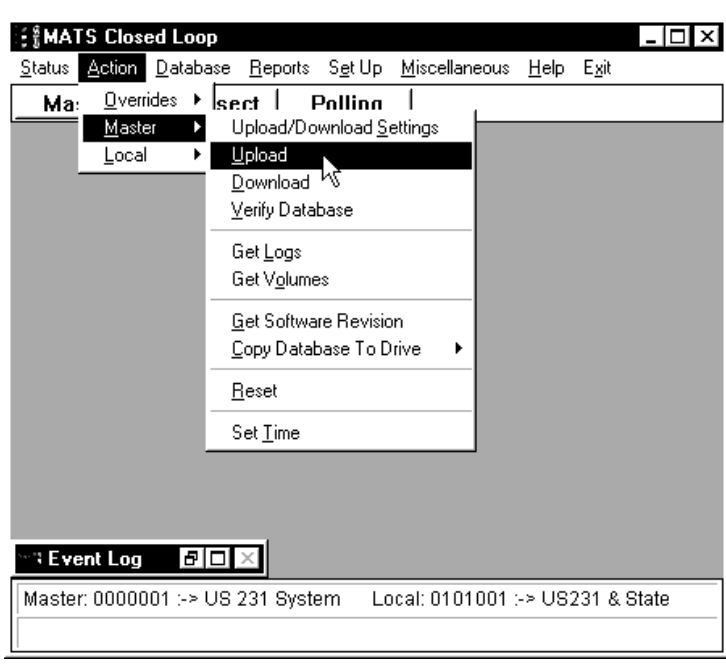

(a) Upload Master

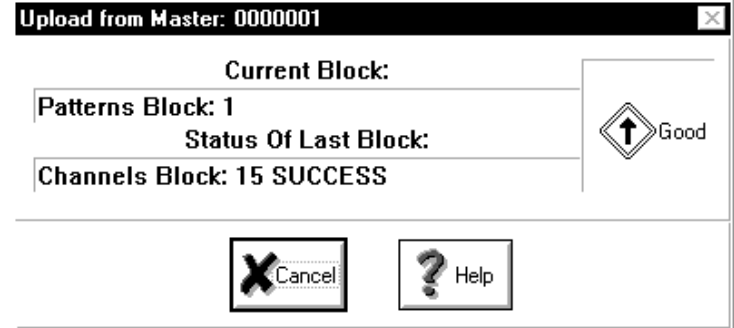

(b) Data Uploading

Figure 4-34 CLMats: Upload Master Database

\subsubsection{Local Upload}

Action $\rightarrow$ Local $\rightarrow$ Upload/Download Settings

In the local controller, all controller data found in the "change data" menu can be modified in

CLMats. However, this is not advised and the data described in Section 4.4.1 should be entered first. The data that is uploaded can be selected in the upload/download options menu shown in Figure 4-35. All data should be uploaded so that the database in CLMats is consistent with the database in the controller. 


\begin{tabular}{|c|c|c|}
\hline Upload/Download Options & \multicolumn{2}{|r|}{$\bar{x}$} \\
\hline Controller: & Coordination: & Time of Day: \\
\hline$\checkmark$ Sequence & \multirow{2}{*}{$\begin{array}{l}v \text { Operating Modes } \\
v \text { Cycle Lengths }\end{array}$} & $v$ TOD Events \\
\hline$\checkmark$ Funct Enable/Start-Up & & \multirow{2}{*}{\begin{tabular}{l|l}
$v$ & Circuit Plans \\
$v$ & Week Plans
\end{tabular}} \\
\hline$v$ Timing Plan Functions & $\checkmark$ Dwell/Min Cycles & \\
\hline$v$ Dynamic Omits & $v$ Offset/oos & \\
\hline$\checkmark$ Dynamic Recalls & & $\checkmark$ Exception Days \\
\hline$v$ Conditional Service & & \\
\hline$v$ Dual Entry & $v$ Permissives & \\
\hline \multirow{2}{*}{$v$ Timing-Init/Clearance } & V Split Matrix & $v$ Sync Reference \\
\hline & $V$ No Early Release & Preemption: \\
\hline \multirow{2}{*}{$\begin{array}{l}v \text { Density } \\
v \text { Overlap }\end{array}$} & \multirow{2}{*}{$\begin{array}{l}v \operatorname{COSF} \text { to TOD } \\
v \text { CS to Timing Plan }\end{array}$} & $v$ Preemption \\
\hline & & Special 0ptions: $\_$All \\
\hline$V$ Standard Overlaps & $v \cos$ to Lead/Lag & \multirow{3}{*}{$\begin{array}{l}\mid v \text { Port Setup / BIU } \\
v \text { Event Call In } \\
v \\
v \text { Output Steering }\end{array}$} \\
\hline \multirow{2}{*}{$\begin{array}{l}v \text { Double Clear Overlaps } \\
v \text { Pedestrian Overlaps }\end{array}$} & $v$ Sync Source & \\
\hline & Special: $\_$All & \\
\hline$v$ Detector Data & \multirow{2}{*}{$\begin{array}{l}v \text { Security Codes } \\
v \text { Intersection Name } \\
v \text { Phone Numbers }\end{array}$} & \begin{tabular}{l|l}
$v$ & Custom Menu \\
$v$ & Restricted Menu
\end{tabular} \\
\hline $\begin{array}{l}v \text { Dimming } \\
v \text { Soft Flash }\end{array}$ & & Select All \\
\hline$v$ Lead/Lag & $v$ EEPROM/Tone & $\underline{\text { Unselect All }}$ \\
\hline & X Cancel & \\
\hline
\end{tabular}

Figure 4-35 CLMats: Local Upload/Download Settings

Action $\rightarrow$ Local $\rightarrow$ Upload

The local database can now be uploaded. If the controller front panel was previously viewed, the upload should not have any errors. After the local database has been uploaded, it can be compared to the existing database in the computer. At this point, the database in the computer is not a valid one and should be overwritten.

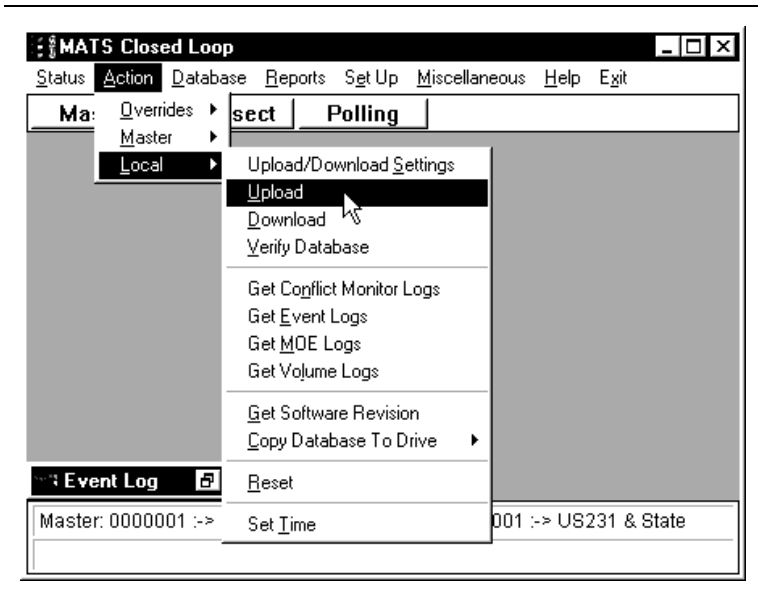

(a) Upload Local

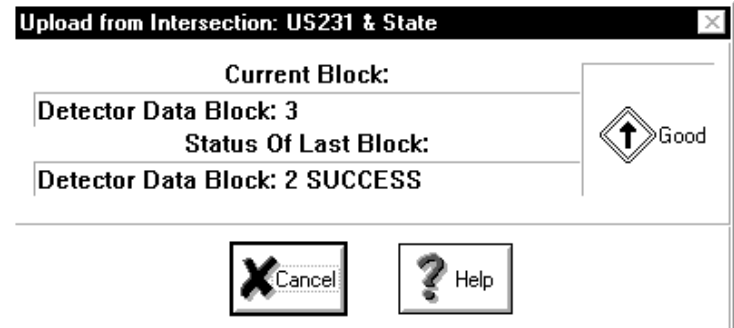

(b) Data Uploading

Figure 4-36 CLMats: Upload Local Database 


\subsubsection{Database Modification}

Database $\rightarrow$ Controller

The menu structure for modifying the database in CLMats is similar to the menu structure found in the controller. As shown in Figure 4-37, the menus correspond, with the exception of the utilities menu in the controller menu (item 6). The CLMats menu also has a few extra menus for computer specific features.

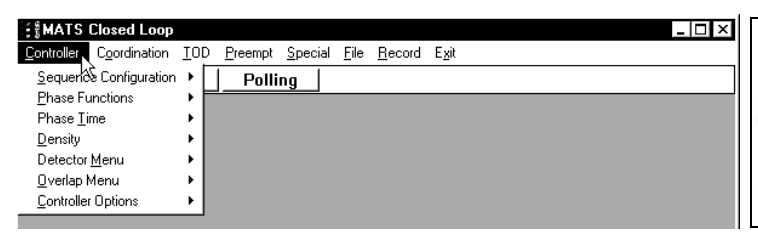

(a) CLMats Menu

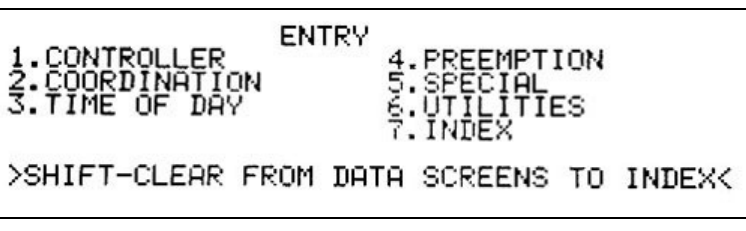

(b) Controller Menu (MM, 3)

Figure 4-37 Local Menu Comparison

\subsubsection{Controller Parameters}

\subsection{Timing Data}

The controller timing data includes the raw data to make the intersection run. The data in these menus should allow the controller to run the intersection in the event that coordination is lost, but the signals are still active. Parameters that must be entered for the intersection to run include minimum green time, maximum green time, yellow clearance, and red clearance. Be careful entering these parameters directly from a Synchro output. Phase green times and phase splits are not the same. Splits are usually entered in percents and therefore total 100 for each ring. Phase splits include green, yellow, and red times for that particular phase. The data calculated in Chapter 2 will be used and is shown in Figure 3-34.

Controller $\rightarrow$ Phase Time $\rightarrow$ Plan 1

In the Peek controller, up to four timing plans can be entered and enabled by a TOD plan. Here, only plan 1 will be used. Note that the table in Figure 4-38a is not in the same sequence as the one shown in Chapter 2. The table in Figure 4-38b outlines the differences in naming conventions for certain parameters between the Econolite controller, Peek controller, and the NEMA TS-2 convention. Figure 4-39 shows the CLMats and controller screens for entering the parameters. 
Item 1 to be entered into the controller is initial green. For each phase in the ring structure, the initial green time should be entered in the corresponding column. Follow the same procedure for items 2-15. Items $a$ and $b$ in Figure 4-39 should be set to 2, so that after 2 consecutive max outs, max 3 will be used and after 2 consecutive gap outs, $\max 1$ or max 2 will be used. Items $c$ and $d$ in Figure 4-40 should be checked to enable density plans and to enable it for certain phases.

\begin{tabular}{|c|c|c|c|c|c|c|c|c|c|c|}
\hline & INT & DESCRIPTION (SEC) & 1 & 2 & 3 & 4 & 5 & 6 & 7 & 8 \\
\hline \multirow{15}{*}{$\begin{array}{l}1 \\
2 \\
3 \\
4 \\
5 \\
6 \\
7 \\
6 \\
9 \\
10 \\
11 \\
12 \\
13 \\
14 \\
15\end{array}$} & 1 & INITIAL GREEN & 5 & 10 & 5 & $\overline{7}$ & 5 & 10 & 5 & 7 \\
\hline & 1 & PASSAGE TIME & 1.4 & 5.0 & 1.4 & 5.0 & 1.4 & 5.0 & 1.4 & 5.0 \\
\hline & 1 & YELLOW CLEARANCE & 3.2 & 4.0 & 3.2 & 4.0 & 3.2 & 4.0 & 3.2 & 4.0 \\
\hline & 1 & RED CLEARANCE & 2.0 & 1.5 & 2.0 & 1.5 & 2.0 & 1.5 & 2.0 & 1.5 \\
\hline & 1 & PEDESTRIAN WALK & -- & 7 & -- & 7 & -- & 7 & -- & 7 \\
\hline & 1 & PEDESTRIAN CLEARANCE & -- & 11 & -- & 11 & -- & 11 & -- & 11 \\
\hline & 1 & MAX1 GREEN & 15 & 46 & 15 & 67 & 12 & 48 & 16 & 66 \\
\hline & 1 & MAX2 GREEN & 15 & 46 & 15 & 67 & 12 & 48 & 16 & 66 \\
\hline & 1 & MAX3 GREEN & 17 & 53 & 17 & 78 & 14 & 56 & 19 & 76 \\
\hline & 1 & MAXIMUM EXTENSION & 1.0 & 3.5 & 1.0 & 5.5 & 1.0 & 4.0 & 1.5 & 5.0 \\
\hline & 1 & TIME BEFORE REDUCTION & -- & 15 & -- & 22 & -- & 18 & -- & 25 \\
\hline & 1 & TIME TO REDUCE & -- & 37 & -- & 54 & -- & 45 & -- & 61 \\
\hline & 1 & MINIMUM GAP TIME & -- & 3.0 & -- & 3.0 & -- & 3.0 & -- & 3.0 \\
\hline & 1 & SEC/ACTUATION & -- & -- & -- & -- & -- & -- & -- & -- \\
\hline & 1 & MAXIMUM INITIAL & -- & -- & -- & -- & -- & -- & -- & -- \\
\hline
\end{tabular}

(a) Controller Parameters from Chapter 2

\begin{tabular}{|c|c|c|}
\hline NEMA TS-2 & Econolite & Peek \\
\hline Minimum Green & Minimum Green & Initial \\
\hline Passage Time & Vehicle Extension & Passage Time \\
\hline
\end{tabular}

(b) Terminology Relations

Figure 4-38 Phase Timings 


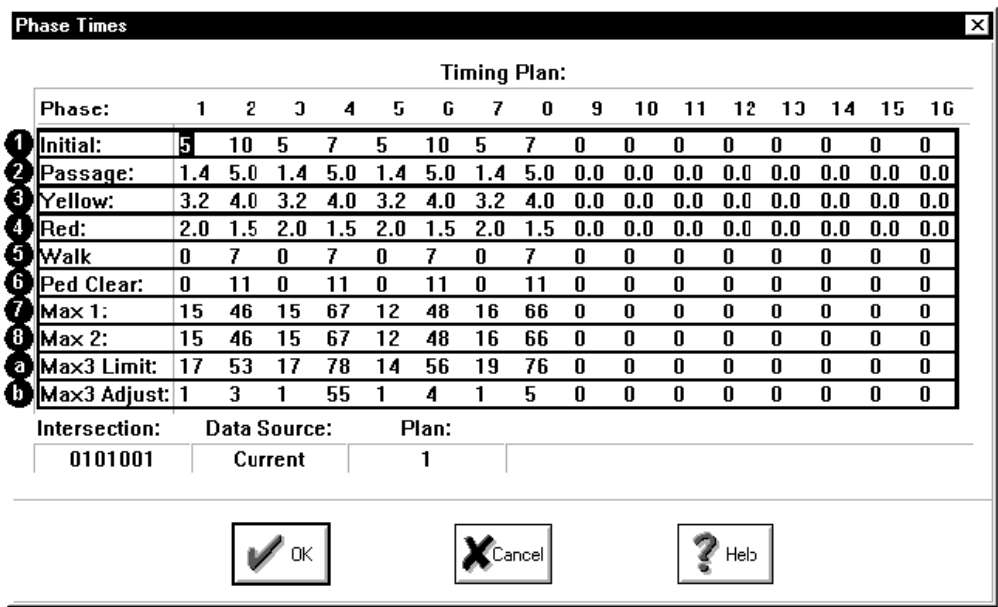

(a) CLMats Timing
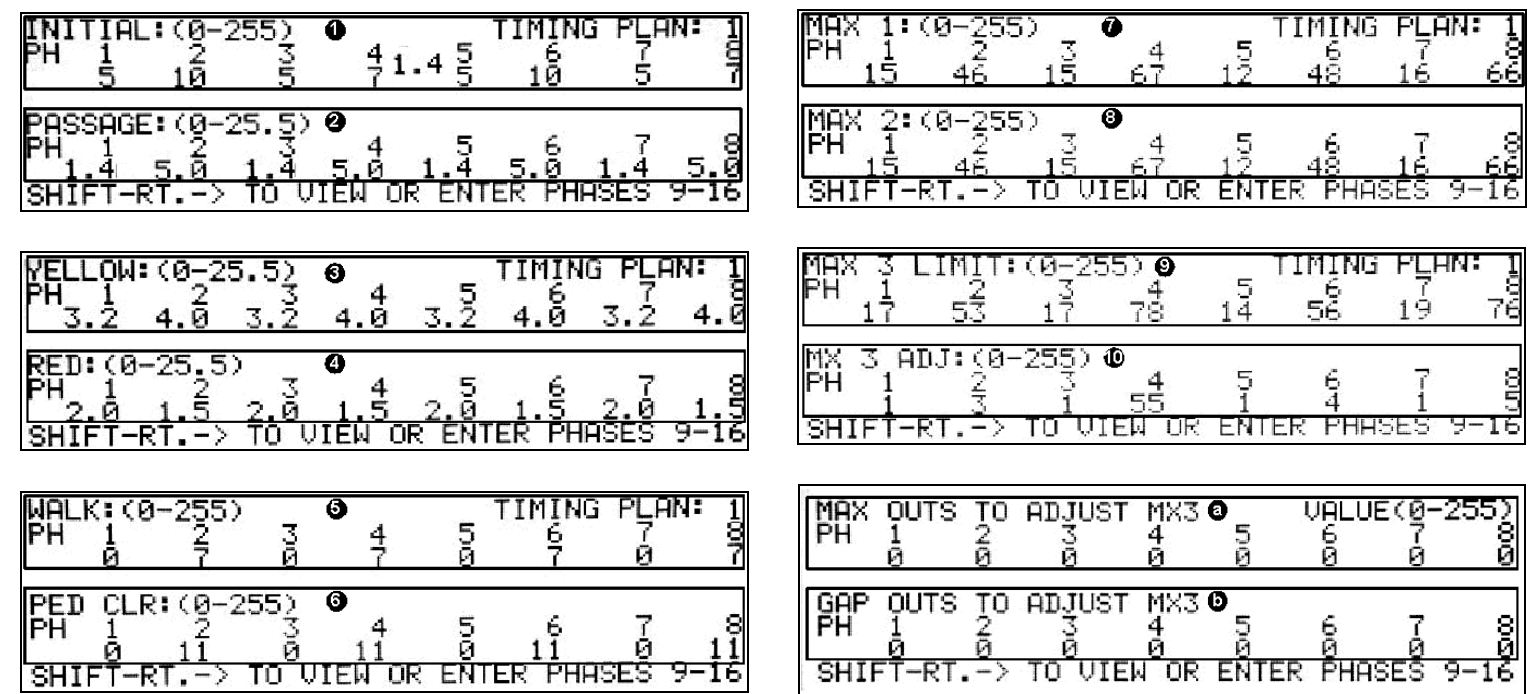

(b) Controller Timings (MM, 3, 1, 3)

Figure 4-39 Phase Timing

Controller $\rightarrow$ Density $\rightarrow$ Timing Plan 1

If gap reduction is used, it is configured in the density menu. Only approaches with speeds greater than 40 miles per hour have gap reduction. Up to four density timing plans can be entered. Figure 4-40 shows the density timing plan screens for intersection 1. Items $\mathrm{c}$ and d allow the user to enable density plans for certain phases. Since the left-turn phases have speeds less than $40 \mathrm{mph}$, density functions are not enabled for them. 


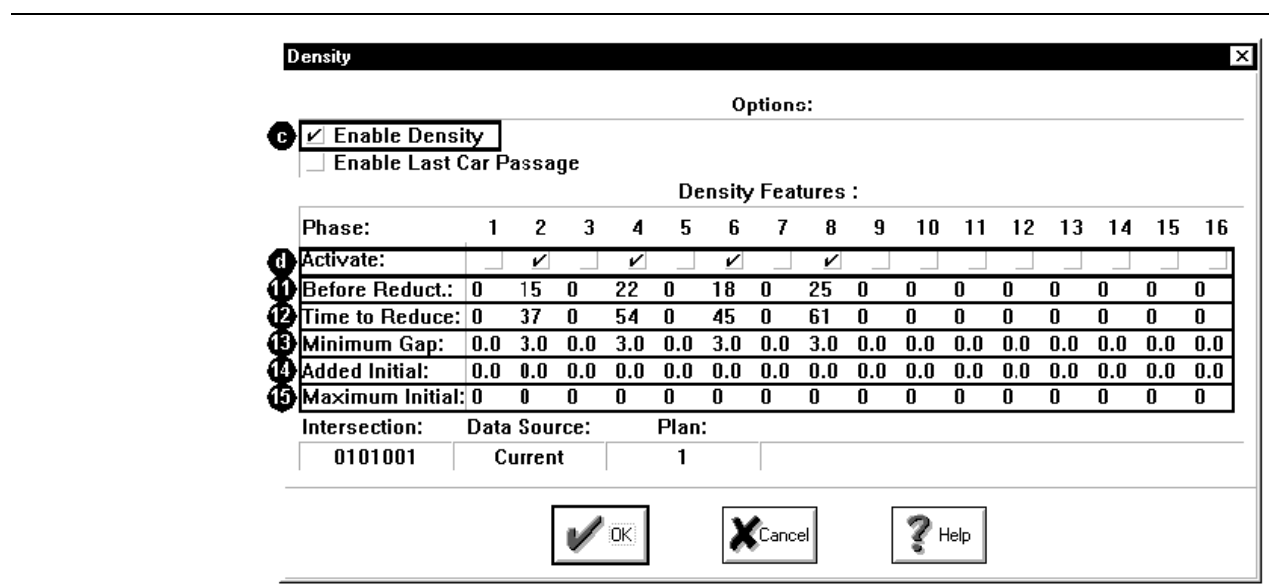

(a) CLMats
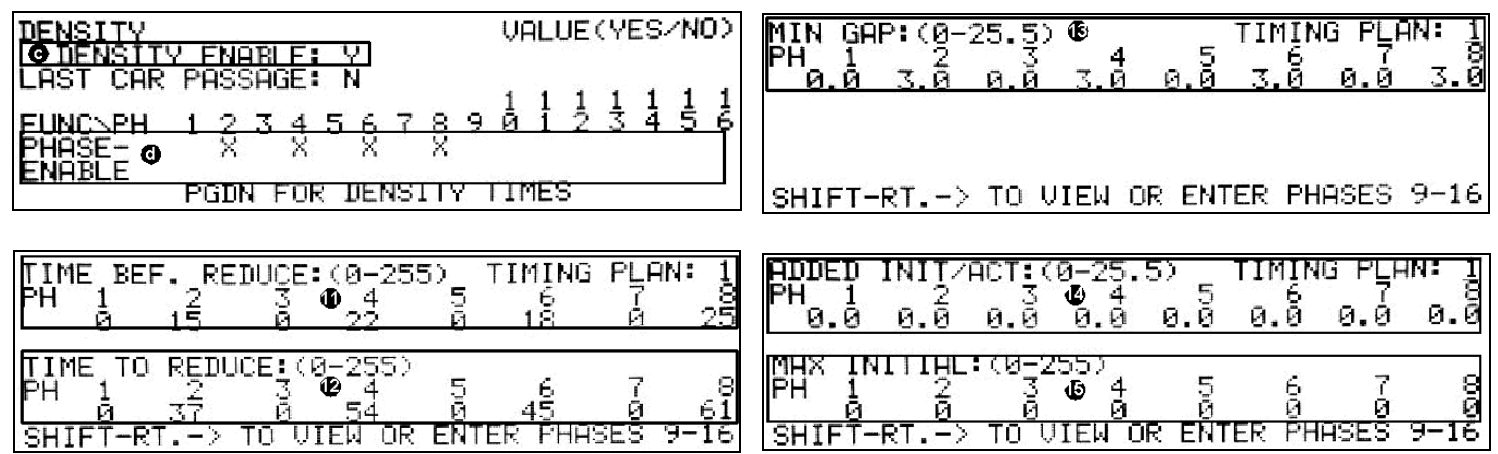

(b) Controller (MM, 3, 1, 4

Figure 4-40 Gap Reduction

\subsection{Recall Data}

Controller $\rightarrow$ Phase Functions $\rightarrow$ Recall Functions $\rightarrow$ Plan 1

Up to four recall plans can be programmed. They are enabled by TOD plans. Minimum recall will place a call on a phase while it is red and the initial green time will be served. Max recall will do the same thing, only once the phase is green, the call remains so that the maximum green time is served. If a movement at the intersection does not have detection, those phases are typically placed in max recall. Ped recall will place pedestrian calls so that the walk and clearance times are served. This might be used at an intersection when the pedestrian buttons are not working or are not installed. It is also used in areas with very heavy pedestrian traffic. If all approaches in the intersection have detection, the main street through phases should be placed in soft recall. This means that once calls are served on the side streets and through movements, the green indication will return to the main street and rest until opposing calls are received. Soft recall would obviously not be needed if the main street phases were in max recall because the green would be returned due to a call. Non-Lock prevents the controller from locking a vehicle call in memory. If a phase is locking, when a vehicle call is received, the call remains until that phase 
has been served, regardless if the vehicle is still actually there. Typically, all detectors should be non-locked. If max 2 times were entered in the timing menu, they could be enabled here.

Otherwise, max 1 times are used.

At intersection 1, phases 4 and 8 do not have detection, as will be seen in Figure 4-57; therefore they will be recalled to their max times. Figure 4-41 shows the recall data screens.

1. In the Max Recall row, place a check in the boxes under the desired phases.

2. In the Non-Lock row, place a check in the boxes under the desired phases.

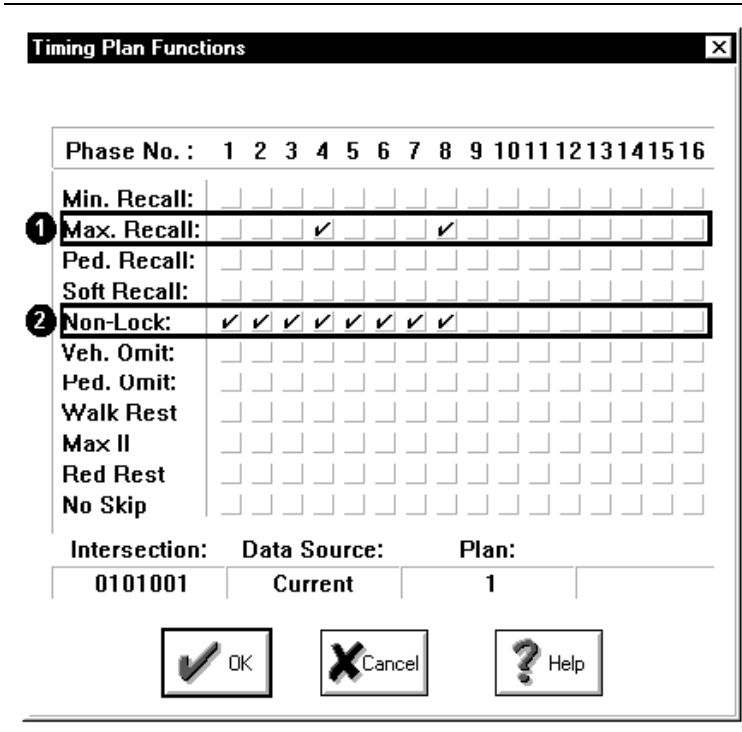

(a) CLMats

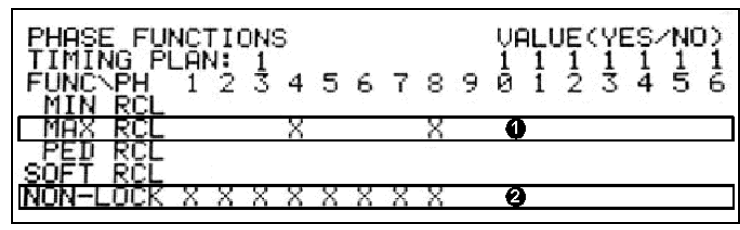

(b) Controller (MM, 3, 1, 2, 5)

Figure 4-41 Recall Data

\subsection{Dual Entry}

Controller $\rightarrow$ Phase Functions $\rightarrow$ Dual Entry $\rightarrow$ Phases 1-8

Dual entry can be configured by phase or in timing plans. The through phases on each approach $2,4,6$, and 8 should be programmed for dual entry. This will prevent the possibility of having only one phase in green. By programming phase 1 for dual entry with phase 6 when phase 1 has a call at an intersection, instead of only phase 1 turning green, phase 6 will also turn green. In the absence of a call in a ring, the dual entry phase in that ring will go green. Dual entry must also be enabled with the check box. The dual entry screens are shown in Figure 4-42. When the phase on the left of the screen is the one that will receive the call. The phases at the top of the screen will activate as the dual entry phase. Here, phase 6 will turn green with either phase 1 or 
2 and phase 2 will turn green with either phase 5 or 6 . This means that a through phase will always be the dual entry phase.

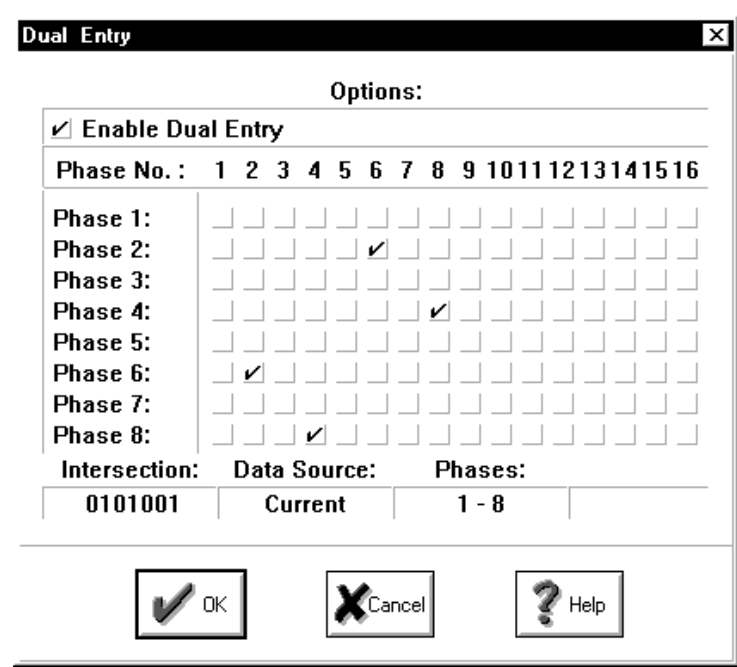

(a) CLMats
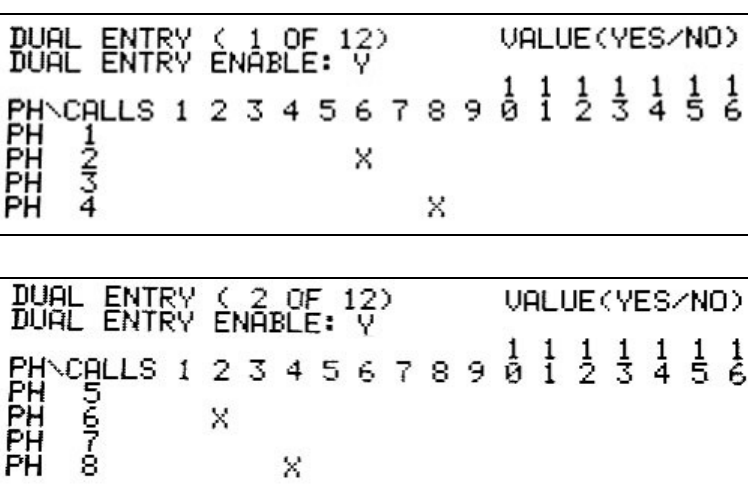

(b) Controller (MM, 3, 1, 2, 4)

Figure 4-42 Dual Entry

\subsection{Simultaneous Gap}

Simultaneous gap is typically enabled at all intersections in Indiana for safety reasons. This feature controls how the phases function when they gap out. Consider phases 2 and 6 in a standard ring structure that must both terminate in order to cross the barrier. If the phase 2 vehicle extension timer expires and phase 6 is still active, with simultaneous gap enabled, the phase 2 vehicle extension timer can be reactivated. Without it enabled, phase 2 cannot be extended again and will terminate when phase 6 gaps out. Simultaneous gap should be enabled for phases 2, 4, 6, and 8 (Figure 4-43). However, when intersections are heavily saturated, more efficient operation can be achieved if this feature is not enabled. 
Dynamic omits are used to omit a phase when another phase is green. Figure 4-44 shows the dynamic omit screen for phase 1 left-turn. Since the left-turn is leading, it will be serviced first, then phase 2. Therefore, phase 1 is omitted while phase 2 is green. Each phase to be omitted has to be programmed in a different plan. For this intersection, there will be a total of four plans one for each left-turn movement.

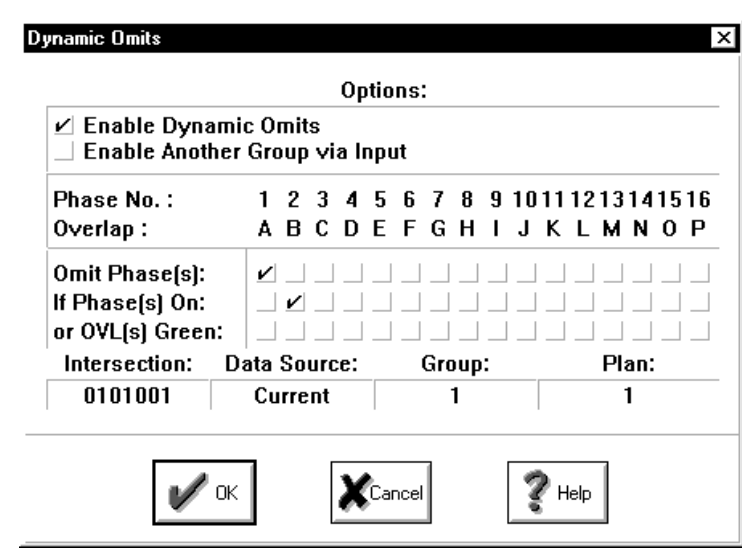

(a) CLMats

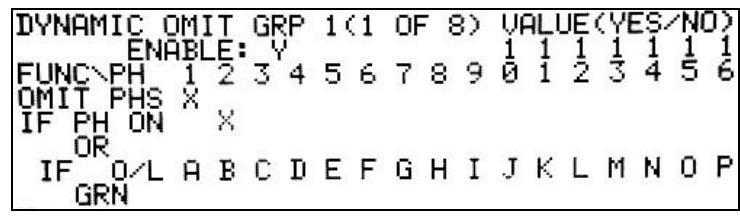
DYNAMIC OMIT GRP $1(1$ OF 8 ) UALUE(YES/NO)

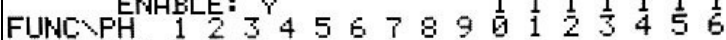
Mif $x$ IF

Figure 4-44 Dynamic Omits

Omitting the left-turn phase using a dynamic omit is not usually a problem, unless the left-turn is protected only, with a 3-section head. During times of very light side street traffic, a left-turning vehicle arriving at the intersection during phase 2 could get stranded because the call for phase 1 would never register because phase 1 is omitted. Generally, this would not be a problem if the left-turn phase were protected/permitted with a five-section head. The only way to service phase 1 again is to cross the barrier into phase 4 and 8 , where phase 1 is active. During light traffic conditions, there may not be any side street traffic to place a call on these phases. Therefore, detector copy can be used to transfer the phase 1 call to phase 4 or 8 while phase 2 is green.

Figure 4-45 shows the phase 1 call being copied to phase 4 while phase 2 is green. Since the call is only copied while phase 2 is green, the copied call will cease once phase 2 goes yellow, ensuring that the copied call cannot max out phase 4 . Since the call goes away on phase 4 , the minimum green time will be served for that phase and then phase 1 will be serviced. When phase 4 is active, phase 8 will also be active because of the dual entry program. If cars arrive while phases 4 and 8 are still green, they will still be able to extend the green. 


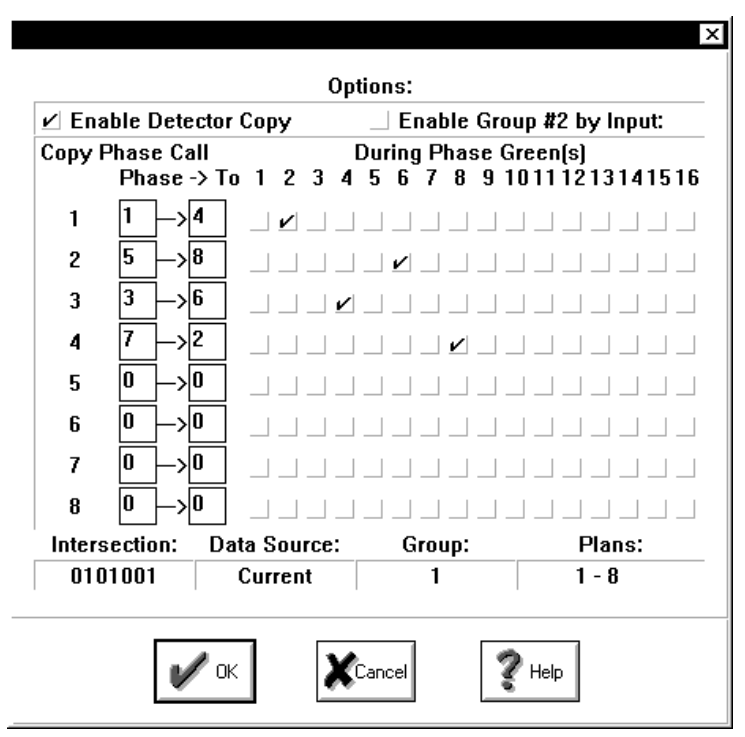

(a) CLMats

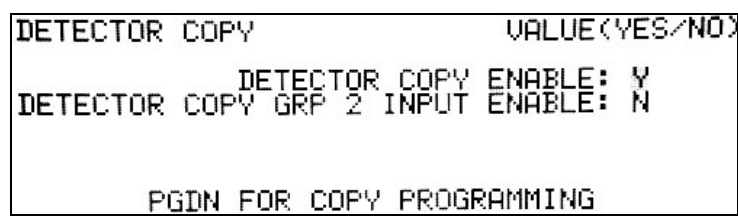

DETECTOR COPW GPF 1216 ON THE FOLLOWING FHASE DIREENS

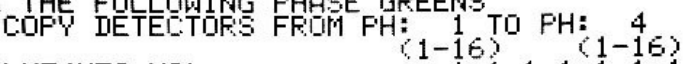
YHL GESES

(b) Controller (MM, 3, 1, 2, 5, 5)

Figure 4-45 Detector Copy

\subsubsection{Coordination}

Coordination plans in Indiana are typically generated by the Synchro design software. Synchro provides cycle lengths, splits, and offsets for given volumes. As was discussed in Chapter 2, a pattern should be generated for the six time periods shown in Table 2-5.

The Synchro outputs for three different coordination plans are shown in Figure 4-47. The CLMats and controller programming screens are shown in Figure 4-48 and Figure 4-49. Before these parameters are configured, the coordination operating modes should be configured as shown in Figure 4-46. The parameters that are not discussed here are not typically used. For more information on these parameters, see the Peek operating manual. ${ }^{24}$

1. Cycle, Split, Offset Source allows the user to decide how coordination plans are selected. If TOD (0) is used, the local controller uses its own TOD plan to place a coordination plan in effect. If Closed Loop (1) is used, the cycle, offset, and split will be selected by the master TOD via the fiber optic interconnect. Interconnect (2) is used for hardwire interconnects and is rarely used. The sources for the cycle, split and offset will be set to closed loop. In the event that the connection to the master is lost, the local controller will revert to its own TOD plan. The TOD plans will be discussed in the next section.

\footnotetext{
${ }^{24}$ Peek 3000 Operating Manual Rev 5.0, Coordination.
} 
2. Free, Flash Source has the same options as the cycle, split, and offset. The free source and flash source should also be set to closed loop.

3. Auto Permissives allows the permissive periods to be calculated automatically. Permissive periods are very important in coordination, however they are difficult to calculate manually. It is always recommended to enable auto permissive calculations, unless an unusual situation requires their adjustment.

4. End of Main Street refers to the offset reference point. If this is not enabled, the offsets are referenced to the latest start of main street green, which are the coordinated phases - usually 2 and 6 . If this is enabled, the offset is referenced from the end of the main street green. It is recommended that end of main street green is used because this point in the cycle is more predictable than the beginning of main street green, due to side street phases gapping out. However, in this example, the reference will be the beginning of main street green, so it should not be enabled.

5. Fixed Force Off determines whether the phase force off points are fixed within the cycle time. If this feature is turned on, extra time in the cycle will be allocated to the side street phases. If this feature is turned off, the extra time will be allocated to the main street. In coordinated systems, the main street usually has the heaviest traffic volumes and should receive the extra green time. However, scenarios exist where the side streets have higher volumes in coordinated systems. This is true for this intersection, so fixed force offs will not be enabled.

6. Offset Entry in \% allows offset data to be entered as percentages, as opposed to seconds. This is not commonly used, but is available.

7. Perm-PA in \% refers to permissives and phase allocations being entered as percentages. Entering phase splits as percentages has a big advantage over phase splits in seconds. If the splits are entered in seconds, a split plan can only be used with cycle length that has the same total time. If percentages are used, there are no problems using a split pattern with different cycle lengths.

8. 4 Splits/Cycle assigns 4 splits per cycle, rather than having 24 total splits that are available to all cycles. This also reduces the number of cycle lengths to 6 . This feature should be enabled.

9. Type of Perm refers to the type of auto permissive operation. The three types are yield, single, and multiple. INDOT typically uses multiple permissives. For more information on permissives, see the Peek Programming Manual ${ }^{25}$.

10. Offset Seeking refers to the transitioning mode when changing coordination patterns. The choices in the Peek controller are add only, dwell, and short route. Add only

\footnotetext{
${ }^{25}$ Peek 3000 Operating Manual Rev 5.0, Coordination.
} 
increases the cycle until the correct offset is obtained. Dwell allows the controller to rest in the coordinated phases until the correct offset is obtained. Short route is the common setting used. The cycle length can be lengthened or shortened to obtain the offset, depending on which would be quicker. Add only and short route are recommended for use.

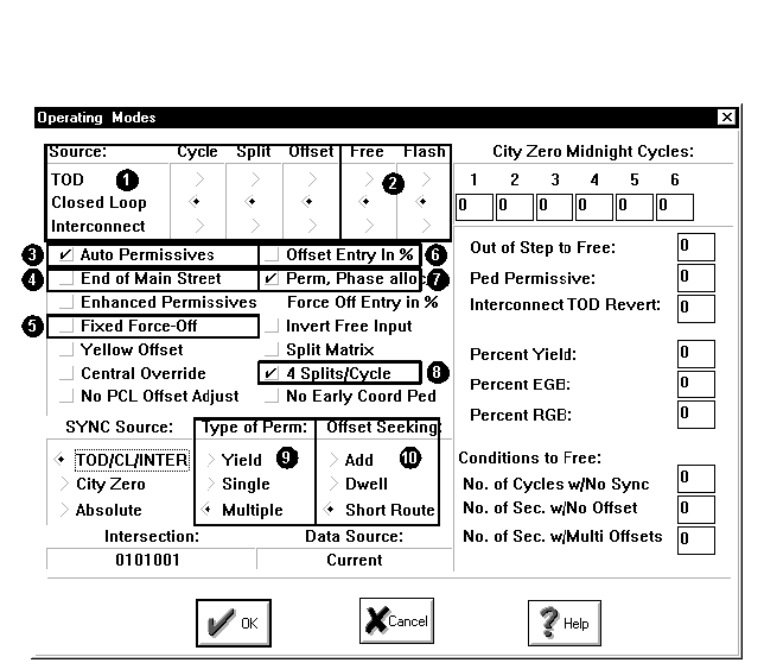

(a) CLMats

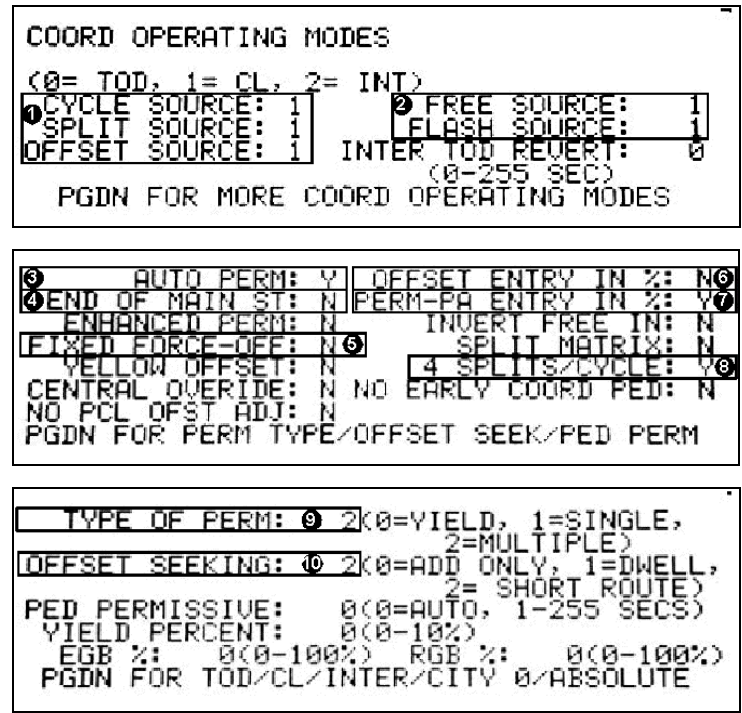

(b) Controller (MM, 3, 2, 1)

Figure 4-46 Coordination Operating Modes

Up to 4 phase split patterns and 5 offsets can be programmed for each of the 6 cycle lengths.

The cycle, offset, and split is enabled by a TOD plan. Typically, various cycles, offsets, and splits would be developed based on different volumes. These different coordination patterns can be selected during a certain time of the day, day of the week, or day of the year.

11. Coordinated Phases are the main street through phases, typically 2 and 6 . Coordinated phases are assigned to each cycle length. This allows the coordinated phases to be switched at different times of the day. Phases 2 and 6 should be configured for the cycles being used.

12. Phase Allocation is the phase splits. Up to 24 split patterns can be entered. If splits are entered in percentages, Ring 1 and Ring 2 phase splits must each total $100 \%$.

13. Cycle Times can be entered from $0-255$ seconds. Max dwell is only configured in this screen if dwell offset seeking was enabled.

14. Offsets can be programmed for each cycle. These numbers are entered as percents or seconds. 
15. $\mathrm{COS} / \mathrm{F}$ to TOD CKTS menu allows the configuration of certain TOD circuits to be active during a coordination plan. The TOD circuits enable timing plans, recall plans, and detector plans. If there is more than one timing plan configured, the desired one can be turned on during certain cycle/offset/split combinations. If a circuit is to be enabled during all coordination plans, a "wild card" value of $7 / 6 / 25 / 1$ is entered. The fourth number in that sequence controls when the circuit will be enabled - 0 for free, 1 for coordination, or 2 for either. If 2 is entered, then the circuit would be active at all times, regardless of coordination. The TOD circuit numbers can be obtained by pressing help in this menu or in the programming manual ${ }^{26}$. Older versions of the manual may not have all possible circuits listed; therefore the help menu screen is the best place to obtain the values. In order to inhibit the max times, two circuits have to be enabled during coordination. These are circuits 105 for ring 1 and 106 for ring 2 . Since the max times are inhibited, phases can only terminate due to a gap out or a force off. Also, TOD circuit 125 needs to be enabled that will log the detector data. This will be on at all times, so 2 is used as the fourth number. Detector logging will be discussed in more detail in Section 4.4.4.4.1.

${ }^{26}$ Peek 3000 Operating Manual, Rev. 5.0, Appendix A. 


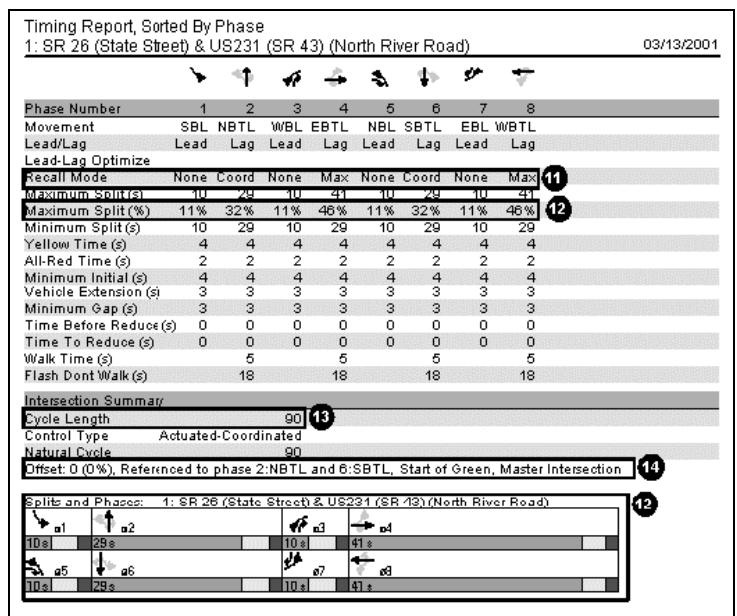

(a) Coordination Pattern 1

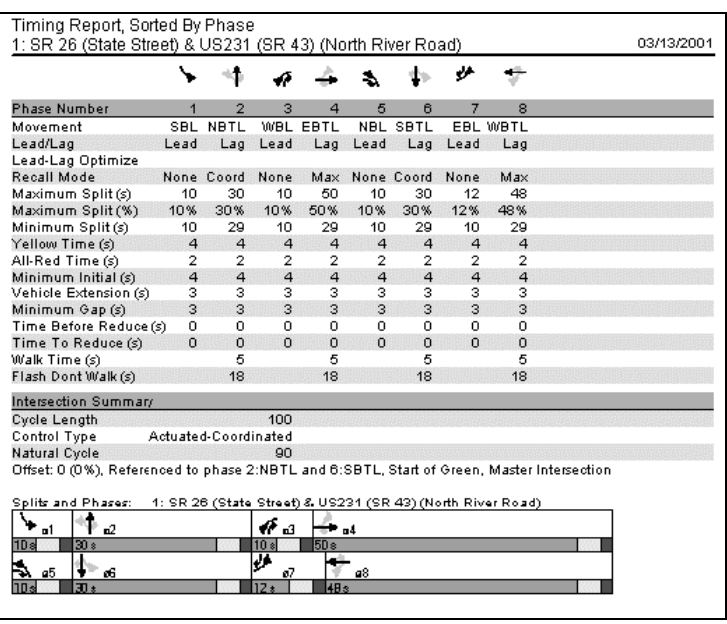

(b) Coordination Pattern 2

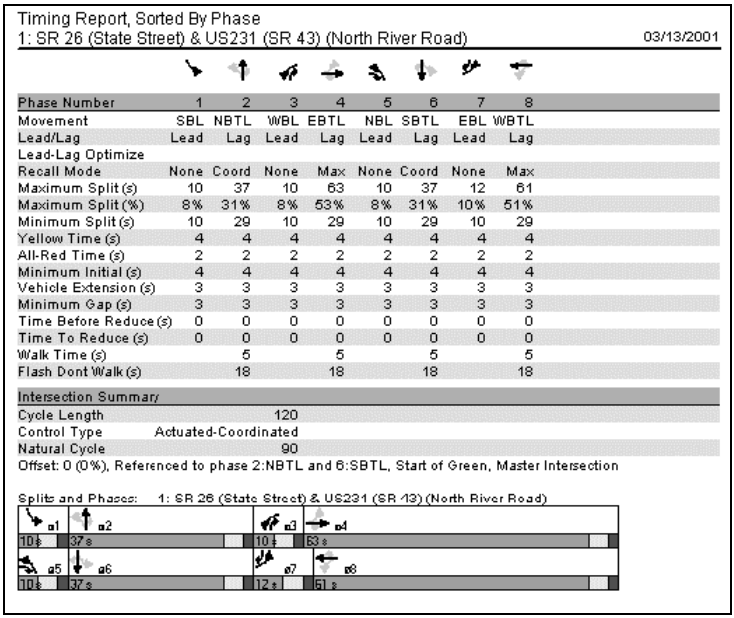

(c) Coordination Pattern 3

Figure 4-47 Synchro Coordination Patterns 


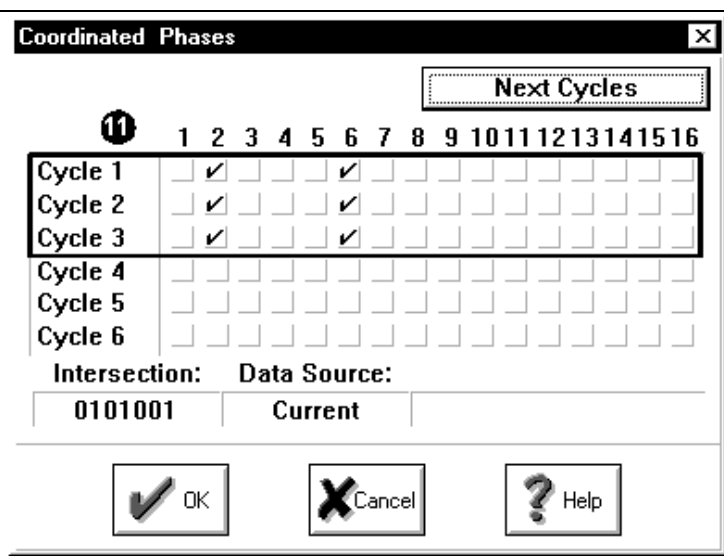

(a) CLMats

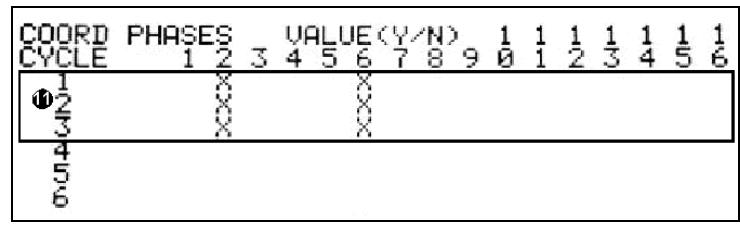

(b) Controller (MM, 3, 2, 2)

Figure 4-48 Coordinated Phases

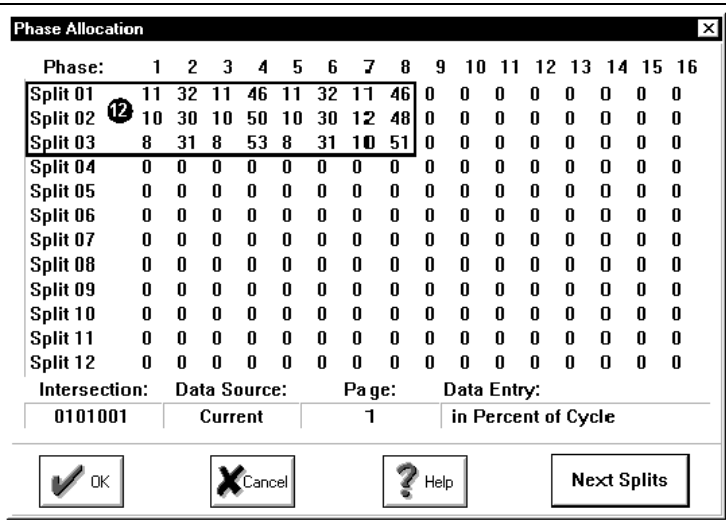

(a) CLMats
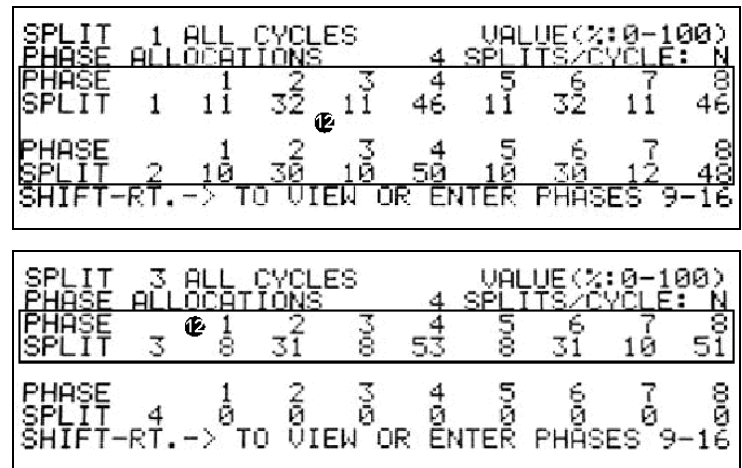

(b) Controller (MM, 3, 2, 3)

Figure 4-49 Phase Split Allocations 


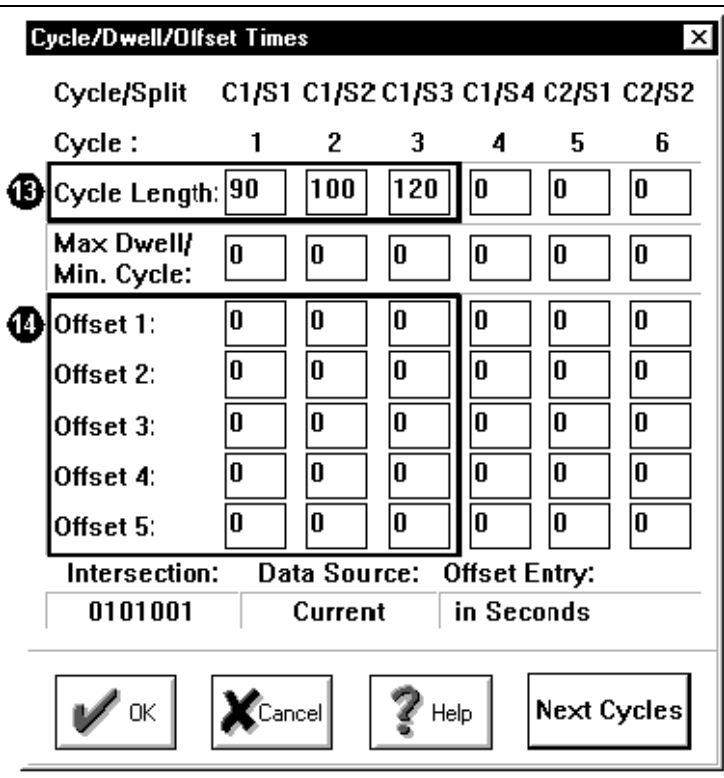

(a) CLMats
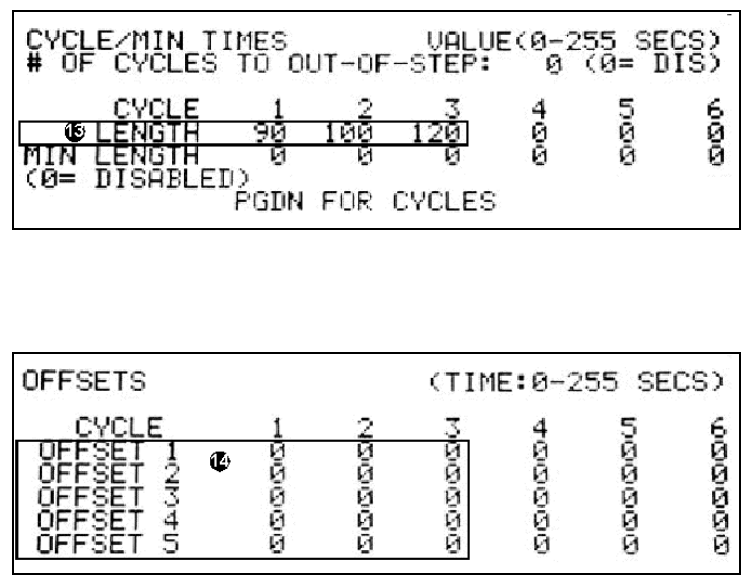

(b) Controller (MM, 3, 2, 5)

Figure 4-50 Cycles and Offsets

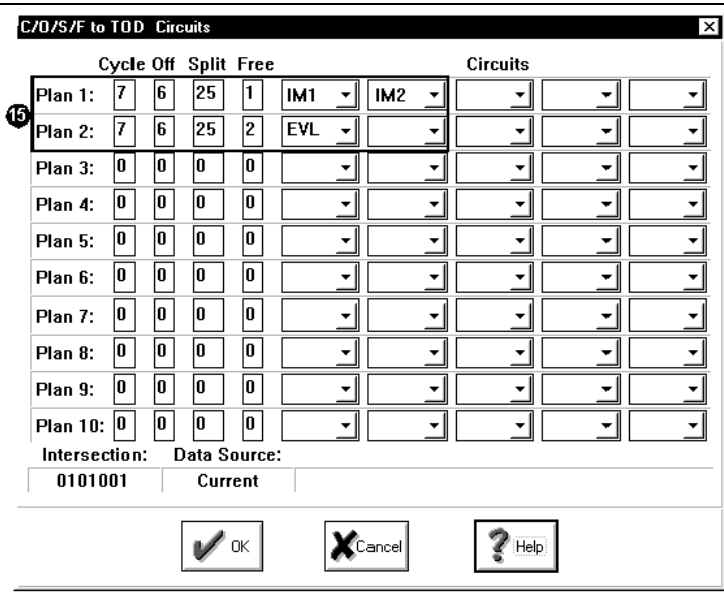

(a) CLMats

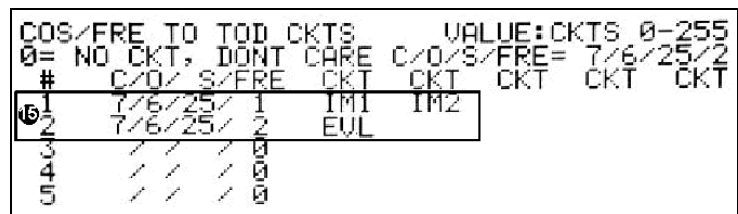

(b) Controller (MM, 3, 2, 8)

Figure 4-51 TOD Circuit in Coordination Plan

\subsection{Check Coordination Plans}

When running a coordinated system, the minimum green times may have to be recalculated.

Consider phase 1 with a minimum green time of 5 seconds. Compare this number to the shortest green time in the coordination patterns for this phase. Since the splits are percentages, the lowest percentage doesn't mean the shortest split in seconds since the cycle lengths vary. The shortest split for phase 1 is 9.6 seconds ( $8 \%$ of 120 seconds) from coordination pattern 3 in 
Figure 4-47. After the shortest split time is determined, the minimum green time can be calculated by subtracting the yellow and red clearance times.

$$
\begin{aligned}
& \text { Phase Minimum Green }=\text { Shortest Split }(\mathrm{s})-\text { Yellow }- \text { Red } \\
& \text { Phase1 Minimum Green }=(8 \% * 120 \mathrm{~s})-3.2 \mathrm{~s}-2.0 \mathrm{~s}=4.4 \mathrm{~s} \leq 5.0 \mathrm{~s} !
\end{aligned}
$$

Since the minimum green time required for coordination is less than the minimum green previously entered, either the minimum green must be decreased or the split increased.

Coordination plans can be checked in the controller. Figure 4-52 shows the controller screens for checking coordination plans 1,2 , and 3 . Plan 2 is ok, but plans 1 and 3 have minimum green errors.
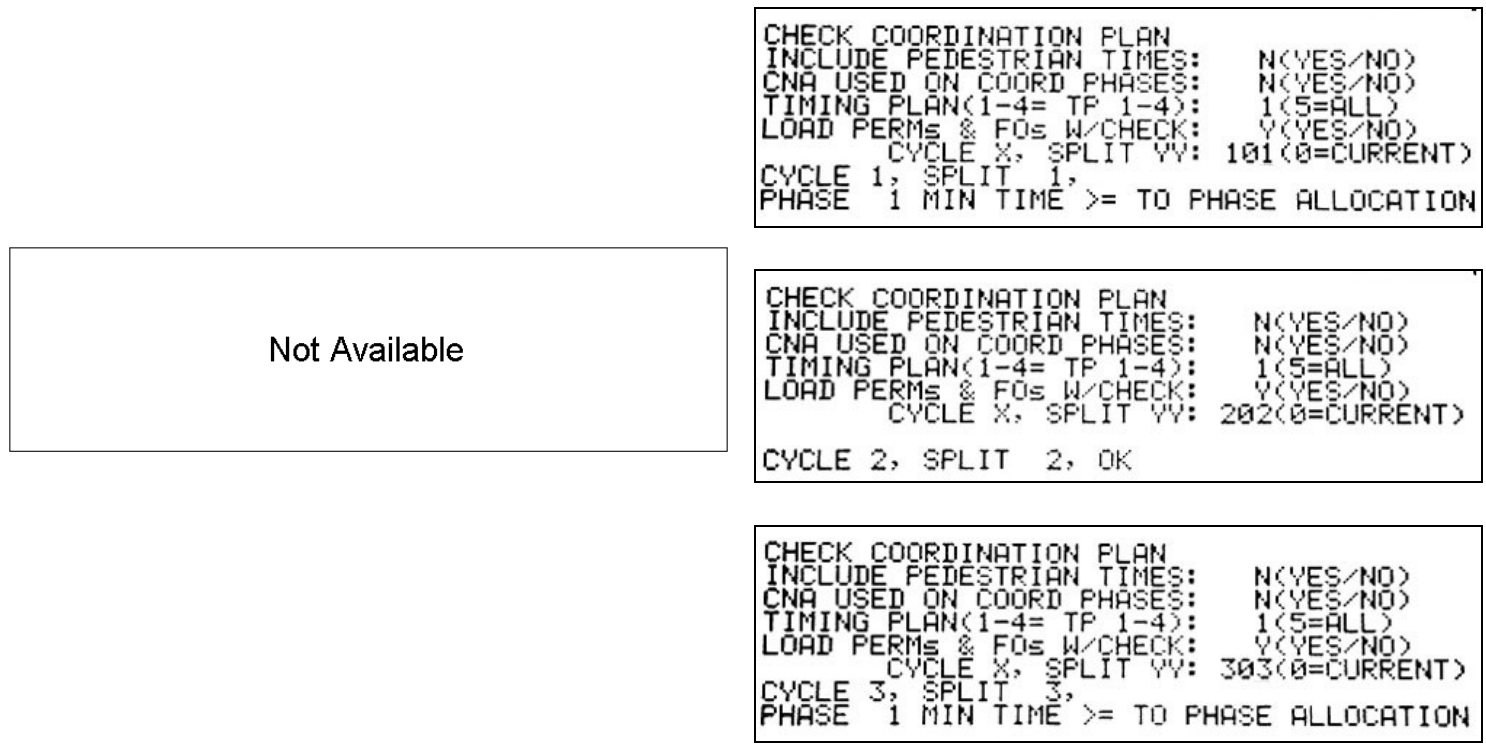

(a) CLMats

(b) Controller (MM, 3, 2, 0)

Figure 4-52 Check Coordination Plans

\subsubsection{Time-of-Day Programs}

Programs can be created in both the master and the local controller. The programs can be run at a certain time of the day, day of the week, or day of the year. Common program purposes include running a certain cycle length, offset, or phase split. If the coordination source is set to closed loop, the local controller will run the coordination plan that the master TOD program indicates. If the signal from the master is lost, the local controller will run the coordination plan 
indicated by its own TOD program. Table 4-2 is the program schedule that will be implemented in this example.

Table 4-2 Time of Day Program Schedule

\begin{tabular}{|c|c|c|c|c|c|c|c|}
\hline Day of Week & Sun & Mon & Tues & Wed & Thu & Fri & Sat \\
\hline Day Plan Number & 2 & 1 & 1 & 1 & 1 & 1 & 2 \\
\hline 6am-9am & \multirow{4}{*}{$2 / 2 / 2$} & $3 / 3 / 3$ & $3 / 3 / 3$ & $3 / 3 / 3$ & $3 / 3 / 3$ & $3 / 3 / 3$ & \multirow{4}{*}{$2 / 2 / 2$} \\
\hline 9am-3pm & & $2 / 2 / 2$ & $2 / 2 / 2$ & $2 / 2 / 2$ & $2 / 2 / 2$ & $2 / 2 / 2$ & \\
\hline $3 p m-6 p m$ & & $3 / 3 / 3$ & $3 / 3 / 3$ & $3 / 3 / 3$ & $3 / 3 / 3$ & $3 / 3 / 3$ & \\
\hline 6pm-10pm & & $1 / 1 / 1$ & $1 / 1 / 1$ & $1 / 1 / 1$ & $1 / 1 / 1$ & $1 / 1 / 1$ & \\
\hline 10pm-6am & Free & Free & Free & Free & Free & Free & Free \\
\hline
\end{tabular}

\subsection{Master Procedure:}

The master TOD programs should match the local TOD programs. Using the master to activate coordination plans is the best way to ensure that all local controllers in the system are running the same plan. If the local controllers do not run the same plans, the system is not coordinated.

1. There are two different day plans in this system. Figure 4-53a \& b show the step beginning at 6:00am in day plan 1 . Figure $4-53 c \& d$ show the step beginning at 10:00pm in day plan 2. The first day plan runs Monday-Friday. The second day plan runs Saturday and Sunday.

2. The hour and minute indicate when that particular step will take effect. In Figure 4-53a \& b, the step shown enables coordination pattern 333 at 6:00 am.

3. The steps in the day plans can be programmed to affect certain zones. Since only one zone is used in this example, all steps will have an ' $X$ ' under ' $Z 1$ '.

4. The cycle, offset, and split are selected corresponding to the cycle, offset, and split in the local controller. If the step enables free operation, like the one shown in Figure 4-53c \& $d$, the box is checked next to "free".

5. This display is only available in the controller and tells the user whether they are editing (Figure 4-53b) the current step or inserting (Figure 4-53d) a new one. To toggle between the two modes, use the SHIFT key. 


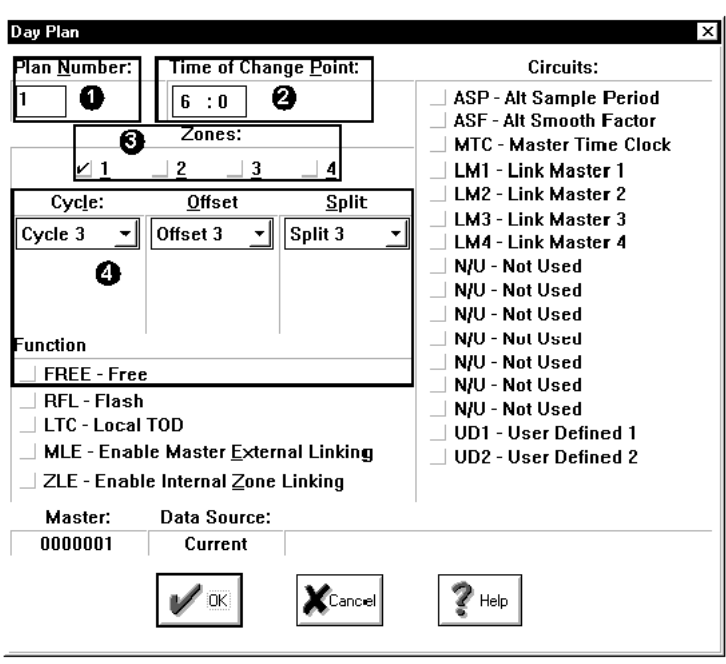

(a) CLMats Day Plan 1 Step

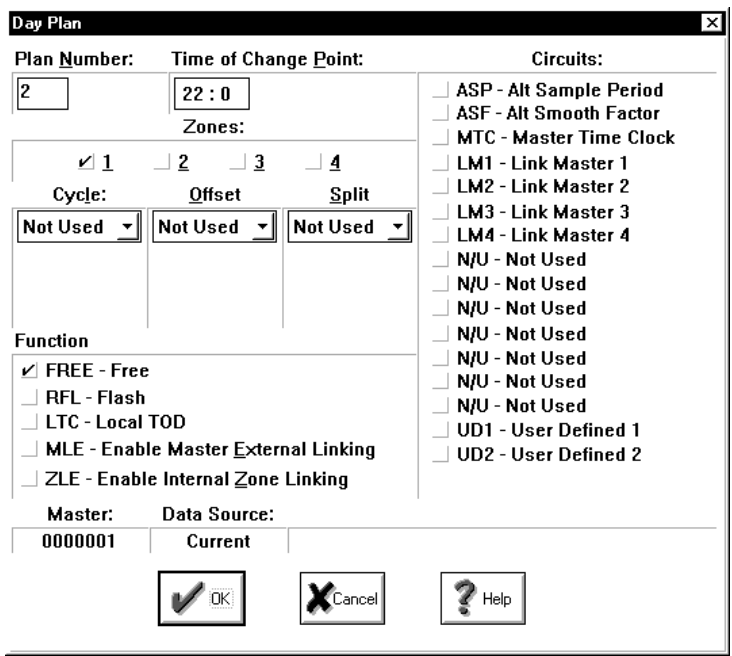

(c) CLMats Day Plan 2 Step

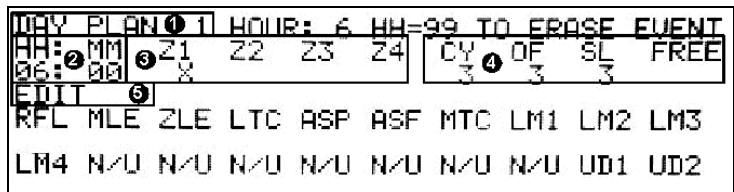

(b) Controller Day Plan 1 Step (MM, 3, 3, 2)

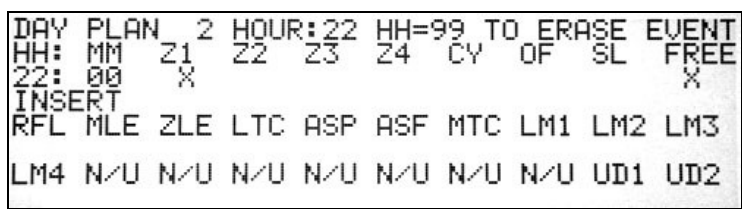

(d) Controller Day Plan 2 Step (MM, 3, 3, 2)

Figure 4-53 Master Time-of-Day Configuration

6. Programs can be enabled on a certain day of the week. In Figure 4-54a \& b, program 1 runs Monday - Friday and program 2 runs on Saturday and Sunday.

7. For each week of the year, a different weekly plan can be activated. The week program number can be enabled based on the week of the year in the menu shown in Figure $4-54 c$ \& d. Since only one week plan is used, nothing needs to be entered here. 


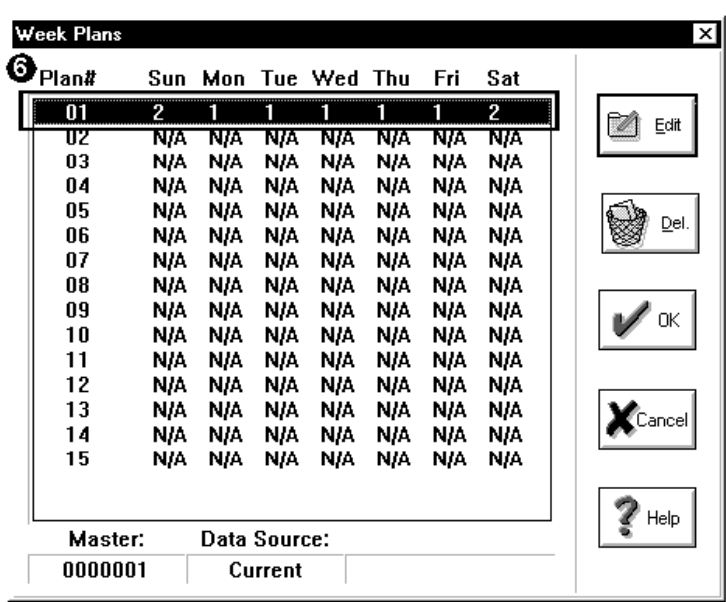

(a) CLMats Master TOD Week

\section{Assign Week Plans}

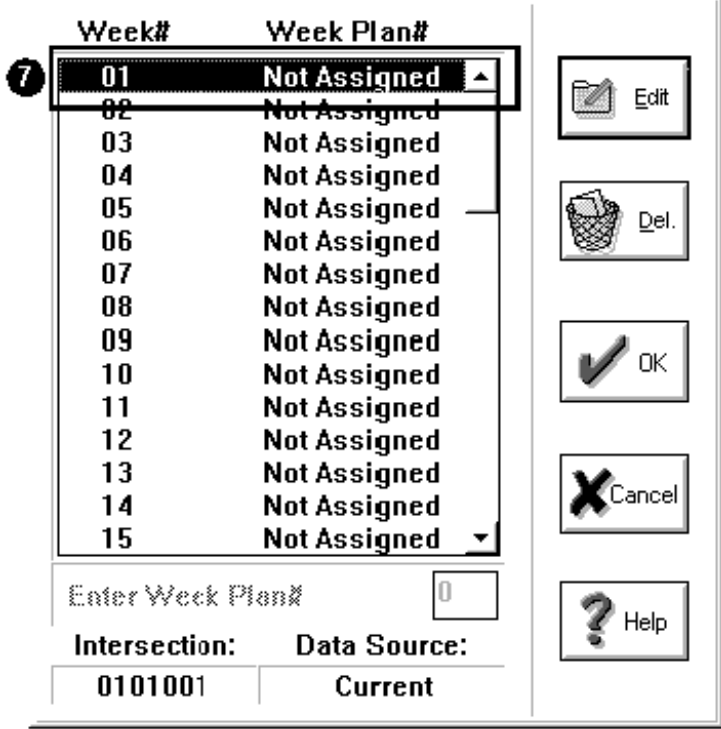

(c) CLMats Master TOD Year

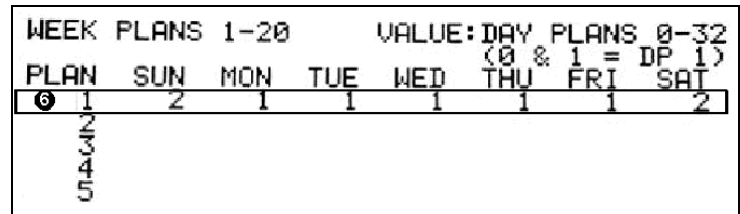

(b) Controller TOD Week (MM, 3, 3, 3)

\begin{tabular}{|c|c|c|c|}
\hline YEAR PLAN & & E: WEE & LANS $1-15$ \\
\hline 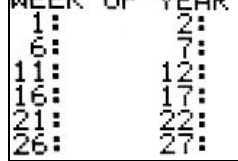 & $\begin{array}{l}3: \\
\frac{1}{3}: \\
28\end{array}$ & $\begin{array}{r}4: \\
9: \\
149 \\
24: \\
29:\end{array}$ & $\begin{array}{r}5: \\
10: \\
15: \\
25: \\
30:\end{array}$ \\
\hline
\end{tabular}

Figure 4-54 Master Time-of-Day Configuration

\subsection{Local Procedure:}

The local TOD program should match the TOD program in the master. In the event that communication is lost to the master, the local controller will still be running the same plan as the rest of the system. Figure 4-55 shows the assignments for the day plans and Figure 4-56 shows the weekly and yearly programs.

1. The day plan number is located at the top of the page. Up to 32 day plans can be configured. 
2. The hour and minute of the day (military time) is entered that the designated cycle/offset/split will become active. It is recommended to begin each day plan at midnight. The Peek controller resets all circuits at midnight (00:00). By beginning the first plan at midnight, possible errors are avoided.

3. The circuit plan generally does not need to be used here, especially if circuits were activated in the coordination submenu. In Circuit Plan TOD submenu (MM, 3, 3, 2), up to four circuits can be activated in a circuit plan. That circuit plan can then be activated by a TOD plan.

4. The cycle/offset/split that is to be activated at this time is entered here.

5. Individual circuits can be turned on or off in the last column according to the time of day.

6. This display is only available in the controller and tells the user whether they are editing the current step or adding a new one. To toggle between the two modes, use the SHIFT key.

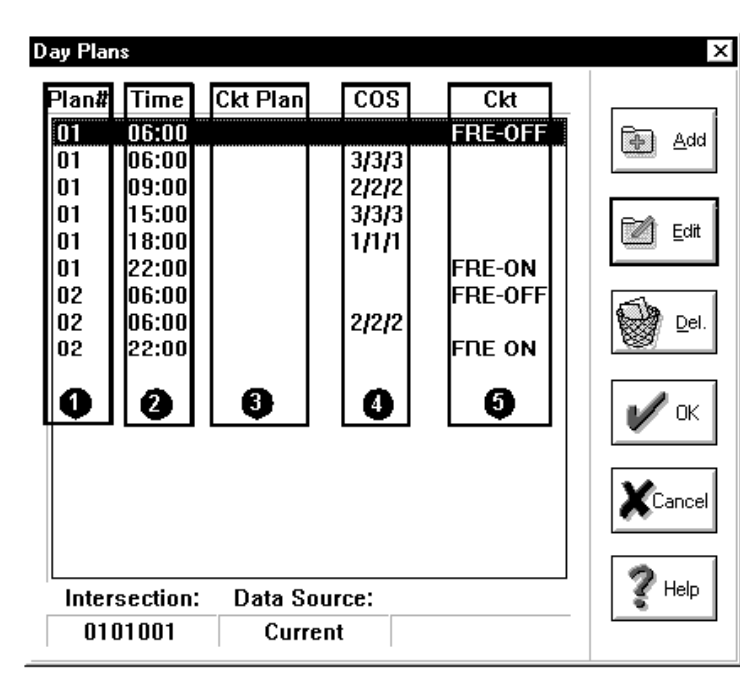

(a) CLMats Day Plans

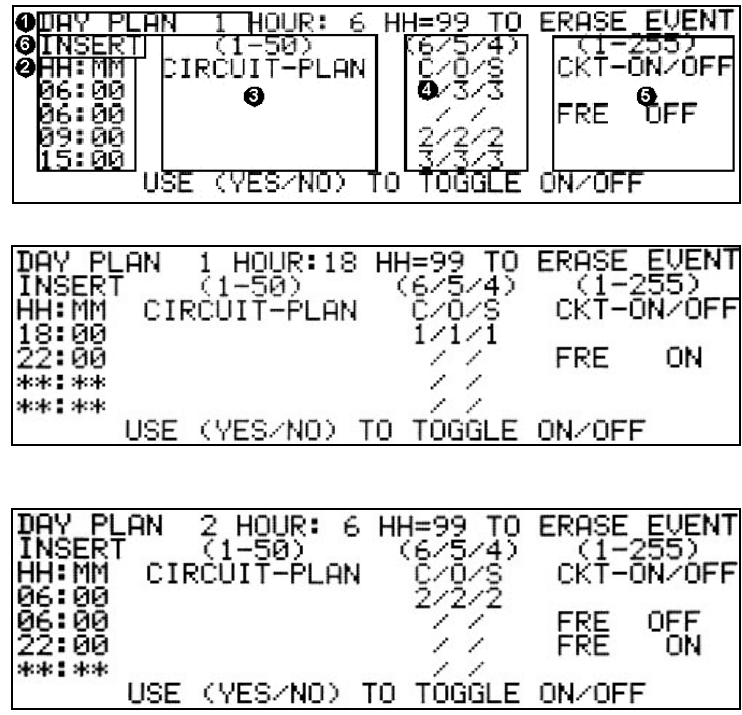

(b) Controller Day Plans (MM, 3, 3, 1)

Figure 4-55 Local Time of Day Configuration

7. For each week plan, the day plan that is in effect on each day of the week is entered. Here, Sunday and Saturday are set to run day program 2, while day program 1 runs Monday-Friday. Only one week program is used here.

8. For each week of the year, a different weekly plan can be activated. Since only one week plan is used, nothing needs to be entered here. 


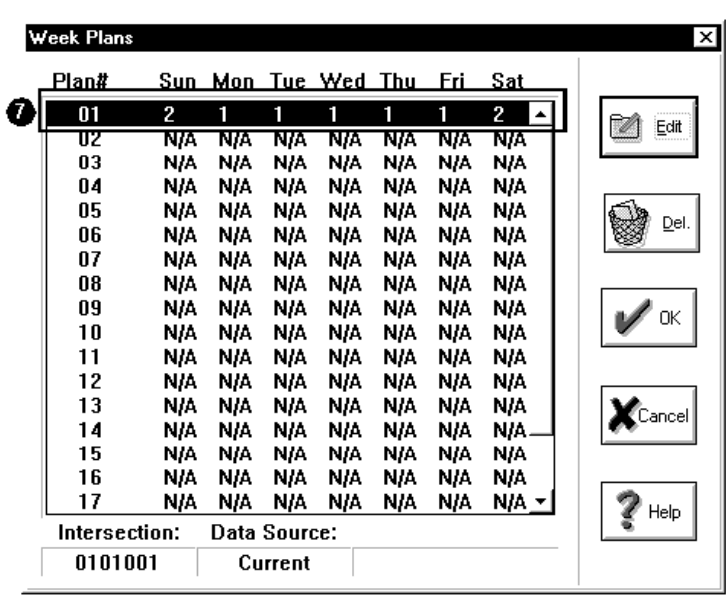

(a) CLMats TOD Week Plan

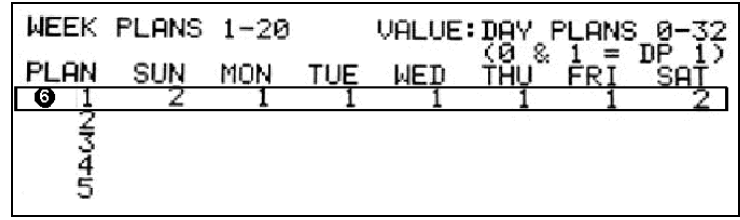

(b) Controller TOD Week Plan (MM, 3, 3, 3)

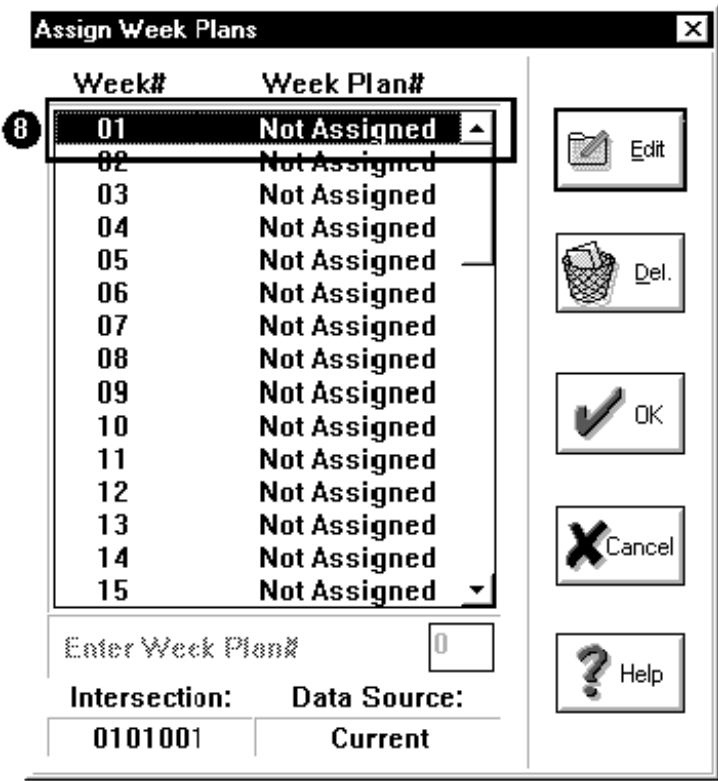

(c) CLMats TOD Year Plan

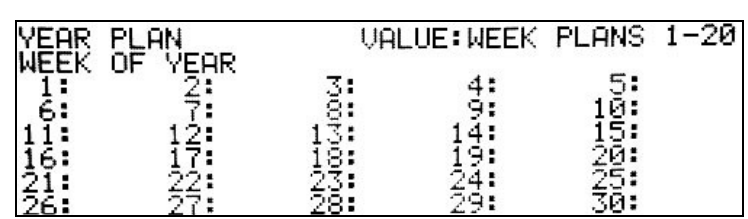

(d) Controller TOD Year Plan (MM, 3, 3, 4)

Figure 4-56 Local Time-of-Day Configuration

\subsubsection{Detectors}

As discussed in Chapter 2, the common detection layout is $3-6^{\prime} \times 6^{\prime}$ loops wired together starting at the stop bar for presence and a fourth loop wired separately for counting. In Figure 4-57a, in the northbound left-left turn lane, detector 5 is a presence detector and detector 15 is a count detector for phase 5 shown earlier in Figure 4-25a. The numbering scheme used here is not the conventional one discussed earlier in Chapter 2 because this is being set up for TS2 Type 2 and was limited to 20 detectors 
Controller $\rightarrow$ Detector Menu $\rightarrow$ Detectors 1-64 $\rightarrow$ Select Detector \#

In the Peek controller, all detectors will be assigned to a phase. All detector data is automatically logged. Logging was enabled by TOD circuit 125 (EVL) in Figure 4-51b. Detectors 18 and 20 are assigned as stretch/delay detectors, or mode 2 in the controller, and a delay time of ten seconds will be entered. These two detectors are in right-turn only lanes. In this situation, when a car activates one of these detectors during the red phase, the call is delayed for ten seconds, in case the car turns right on red. If the car has not accepted a gap within ten seconds, the call is placed. The rest of the detectors are call/extend detectors so that they can call a phase during the red interval and extend the phase during the green interval. In the controller, this is detector mode 0. Figure 4-57 depicts detector assignment in both CLMats and the controller. In CLMats, the detector number is selected, then all information about that detector can be configured.

1. The intersection detectors are assigned placing a check in the box next to the phase that is called. In the controller, detectors are assigned to their corresponding phase by placing an ' $x$ ' in the corresponding row and column. The detector numbers are on the vertical axis and the phase numbers are on the horizontal axis. For example, detectors $2,9,10,12,17$, and 18 are all assigned to phase 2 because that is the phase that they call. Make sure that these detectors are not assigned to any other phases. In general, there should only be one ' $x$ ' in each row.

2. In CLMats, the detector mode is configured in the same screen using a drop-down menu. In the controller, a different screen is used to assign the detector modes. All presence detectors at this intersection are mode 0 - call/extend detectors. However, the two presence detectors in the right-turn lanes, detectors 18 and 20, are assigned mode $2-$ stretch/delay, which enables the extend and delay settings.

3. Up to 4 timing plans can be configured for the delay and extend times. Only plan 1 will be used here. In CLMats, the delay time of 10 seconds can be entered in the appropriate box. In the controller, the screen shown in Figure 4-57e must be accessed to enter the delay times for detectors 18 and 20 . 


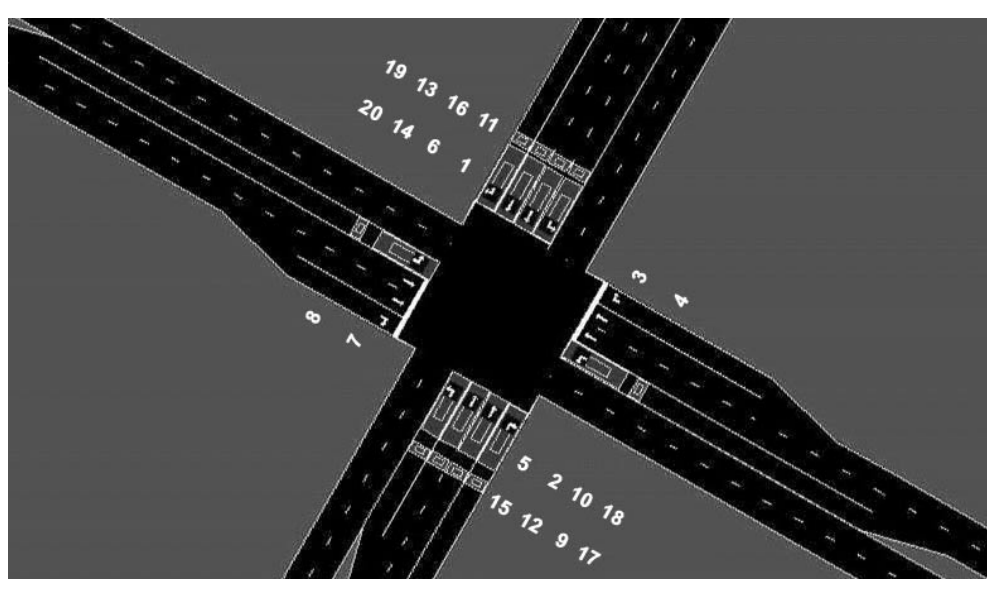

(a) Intersection Detector Layout

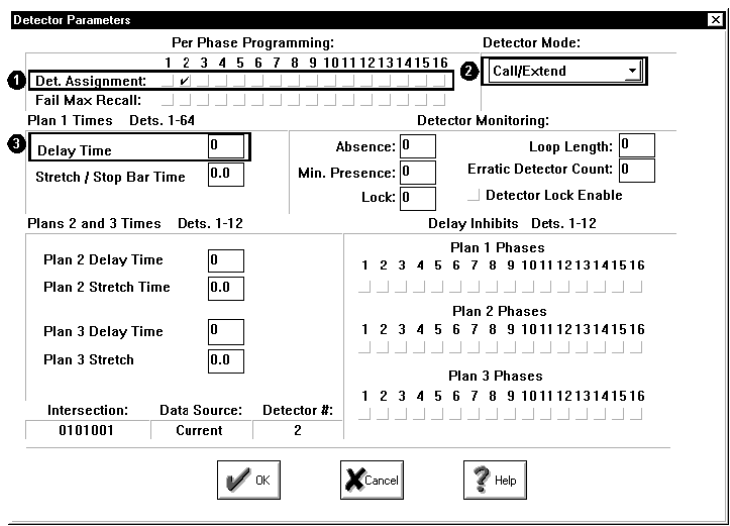

(b) CLMats Detector 2

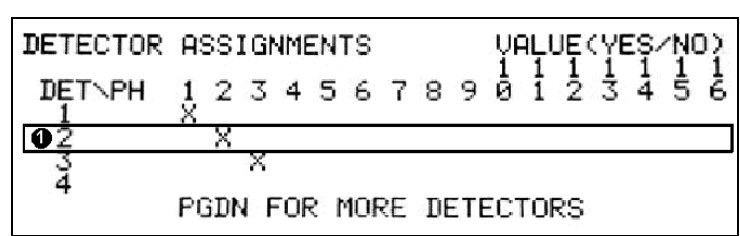

DETECTOR MODE CD-4=CHLL $\angle X X$, CALLING,

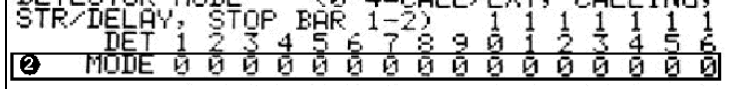

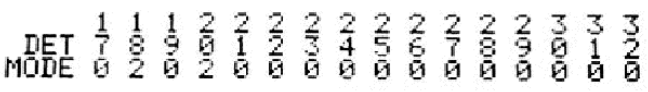

(c) Controller Detector $2(\mathrm{MM}, 3,1,5)$

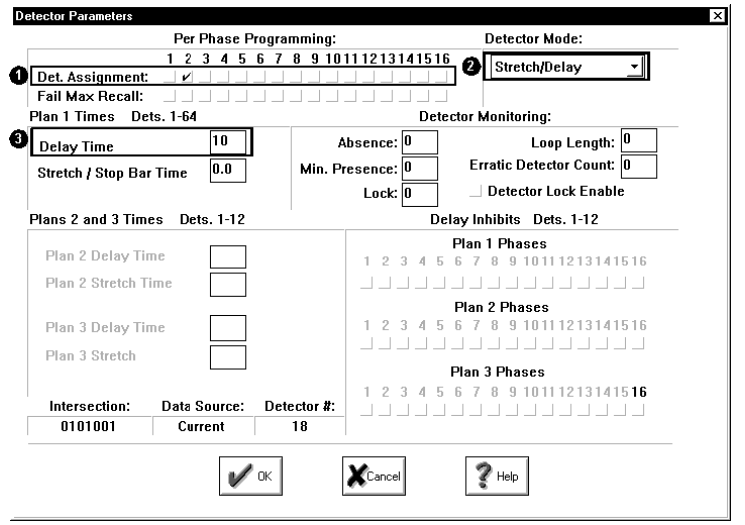

(d) CLMats Detector 18

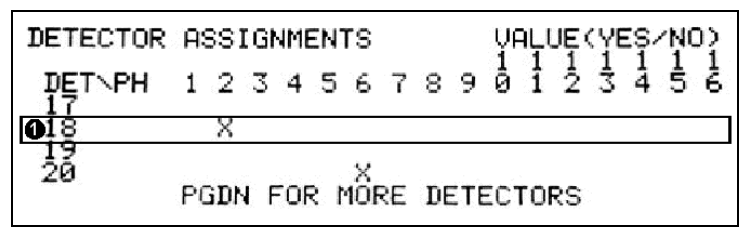
DETECTOR MONE STE

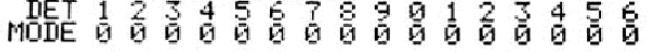

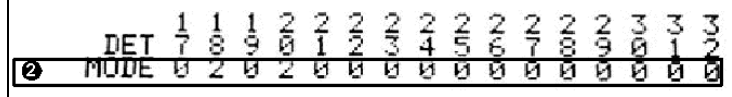

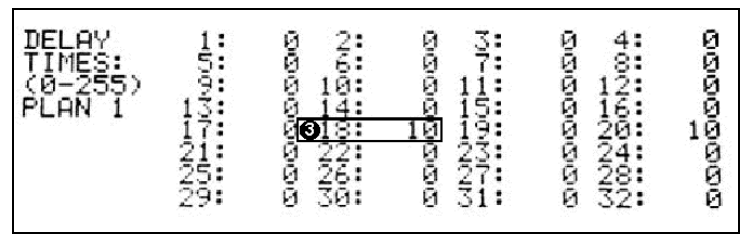

(e) Controller Detector 18 (MM, 3, 1, 5)

Figure 4-57 Detector Assignment 


\subsection{Logging}

Installing count detectors allows the local controller to collect volume data. Since presence detectors are used for counting, volume data as well as occupancy data is collected. Detector logging was enabled by a TOD circuit 125 in Figure 4-51. The data that is logged can either be viewed from the controller or downloaded to CLMats. In the local controller, the log interval needs to be configured as shown in Figure 4-58a \& b. The interval entered must be divisible into 60. Therefore, the interval could be 1, 2, 3, 4, 5, 6, 10, 12, 15, 20, 30, or 60. In Indiana, the interval is set to 60 minutes because hourly volumes are most beneficial for calculating timing plans.

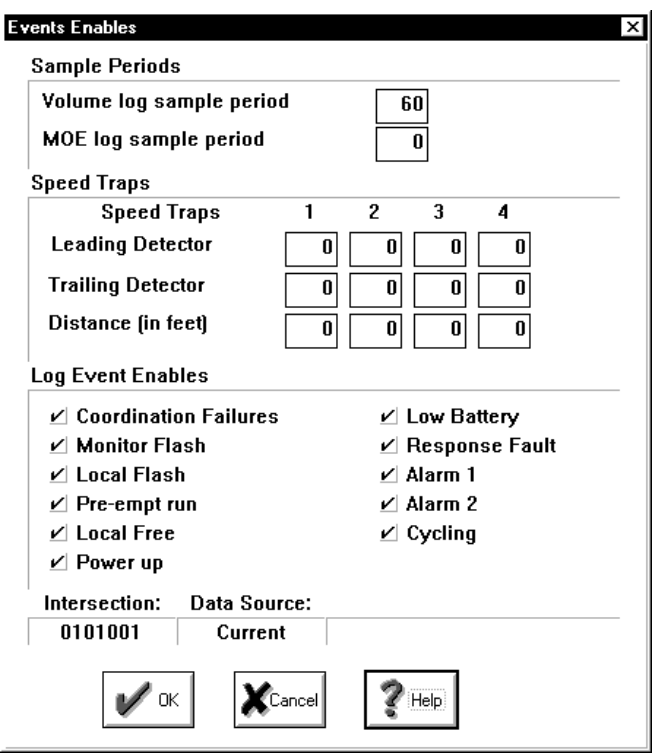

(a) CLMats Log Interval

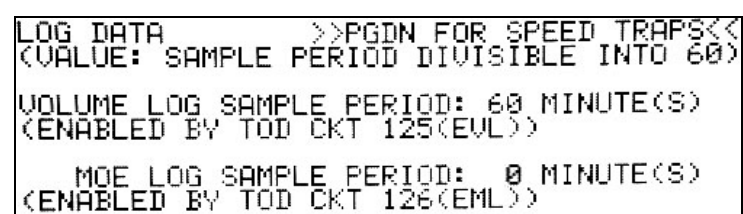

(b) Controller Log Interval (MM, 3, 5, 4, 4)

Figure 4-58 Detector Log Interval

Before downloading the log data, the "Define Basic Elements" screen shown in Figure 4-59a must be configured to avoid getting any errors. Enter the city and state in which the system is located. If this is not configured, the detector log transfer will not work. The procedure for downloading the logs is shown in Figure 4-59b. The data that is collected is stored in the controller memory buffer. This data should be downloaded on a regular basis, usually once a week. Once the buffer is full, previous log buffer data will be overwritten. 


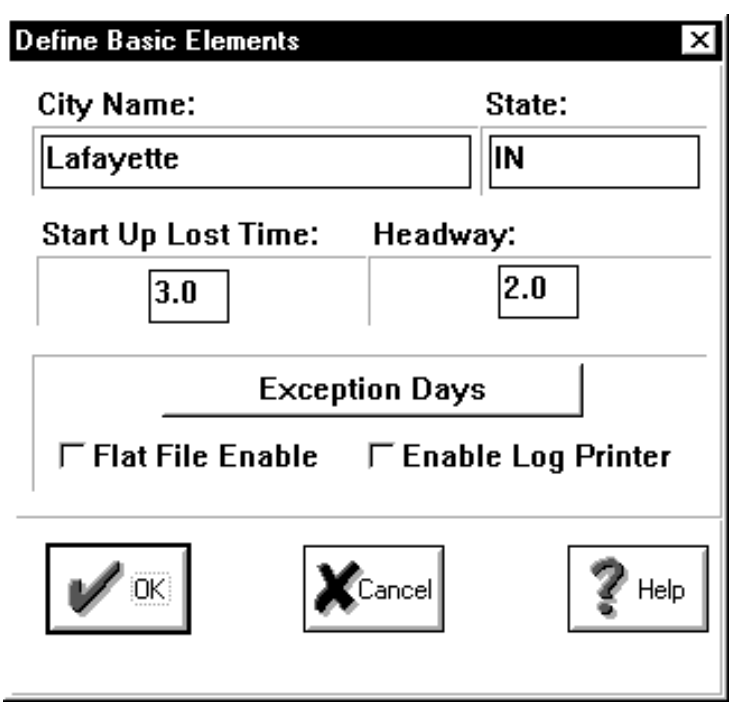

(a) Define Basic Elements Screen

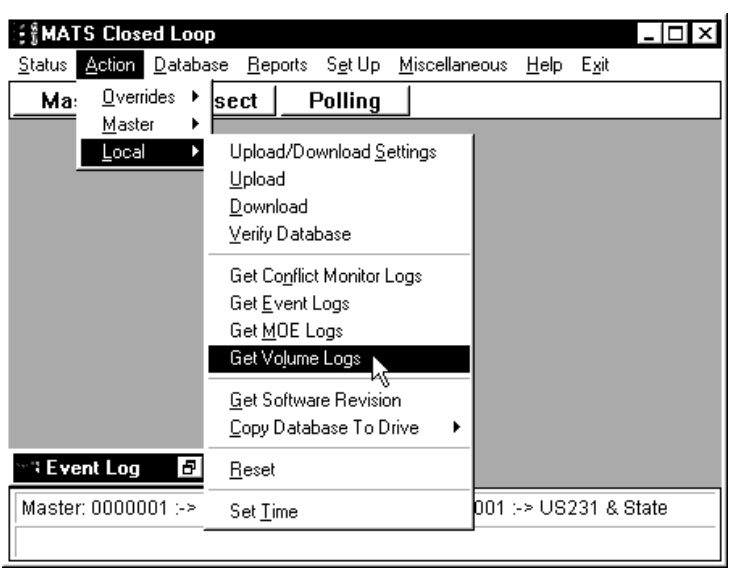

(b) CLMats Download Detector Log

Figure 4-59 Detector Log Transfer

Once the log data is transferred, it can be sorted by date (Figure 4-60b). Once sorted, it can be viewed as a table (Figure 4-60c) or exported to a file. The file exporting should be done from the table view, not the filter screen. The data can be exported in various file types including spreadsheet files and text files. CLMats will store data for each detector in each system every day of the year. The data can also be viewed in the controller as shown in Figure 4-60d. 


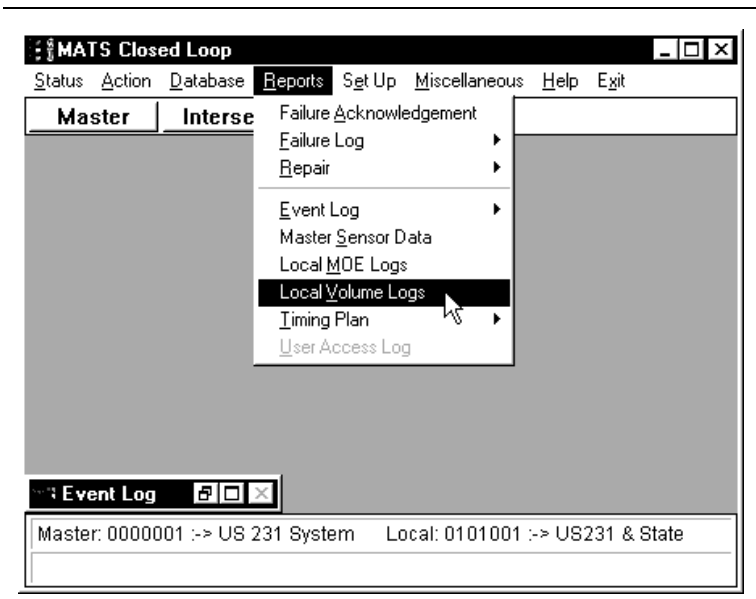

(a) CLMats Log Manager

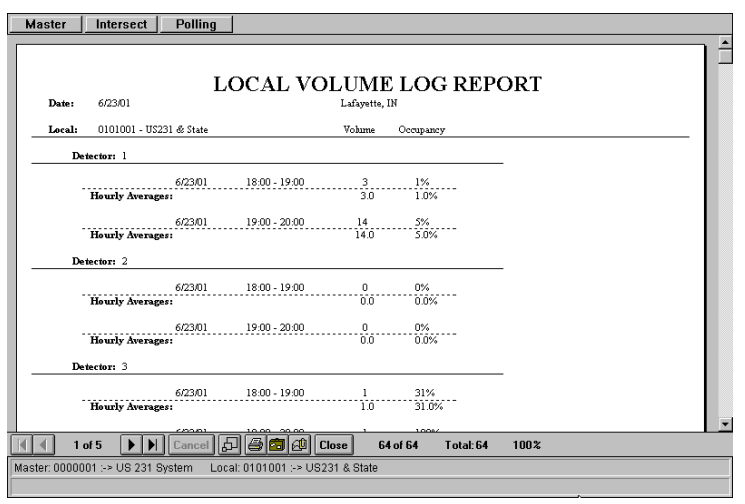

(c)CLMats Log Report

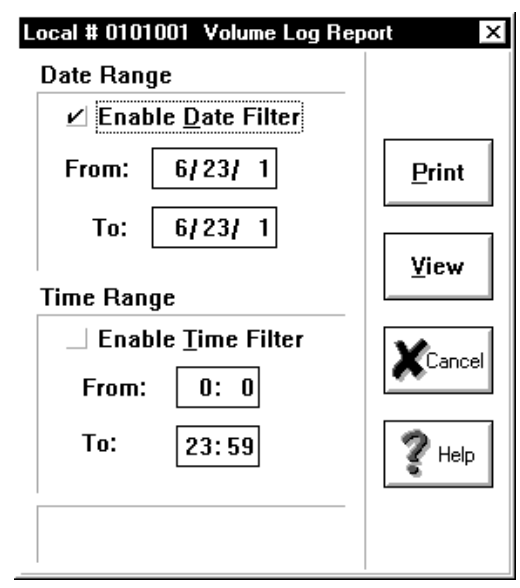

(b) CLMats Log Filter

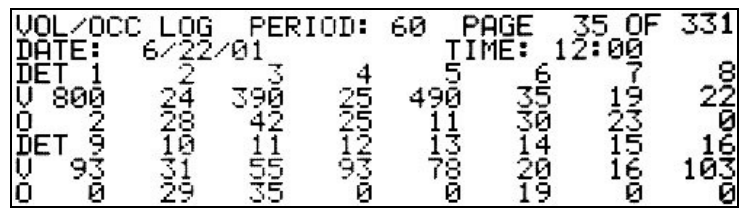

Figure 4-60 Detector Logging Output

\subsubsection{Downloading To Controller}

Once all data has been entered into CLMats, the database needs to be downloaded back to the controller. This needs to be done for both the master and the local controllers. 


Please Wait. Converting Databases...
\begin{tabular}{|l|} 
Detector Data \\
\hline Cancel
\end{tabular}

(a) Converting Databases

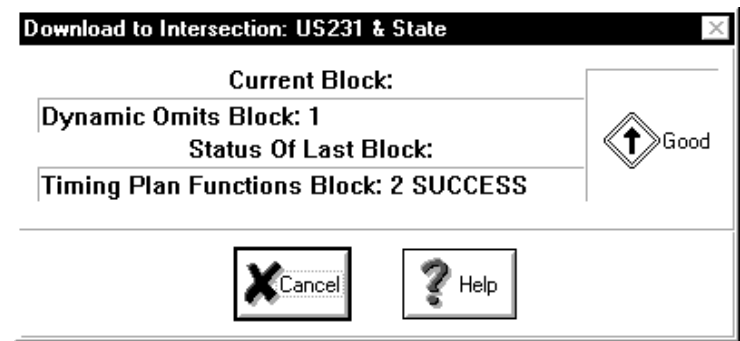

(b) Transferring Databases

Figure 4-61 Download Databases

\subsubsection{CLMats Graphics}

The controller front panel display has already been demonstrated in this section. Another very helpful feature of CLMats allows the user to see a graphic display of the controller state on the computer. Graphics can be drawn or actual pictures can be used like the one shown in Figure 4-62. They are overlaid with images in CLMats and the images are assigned to a phase or a detector. The signals display the phase indications and the colors of the detectors will turn blue when they are active.

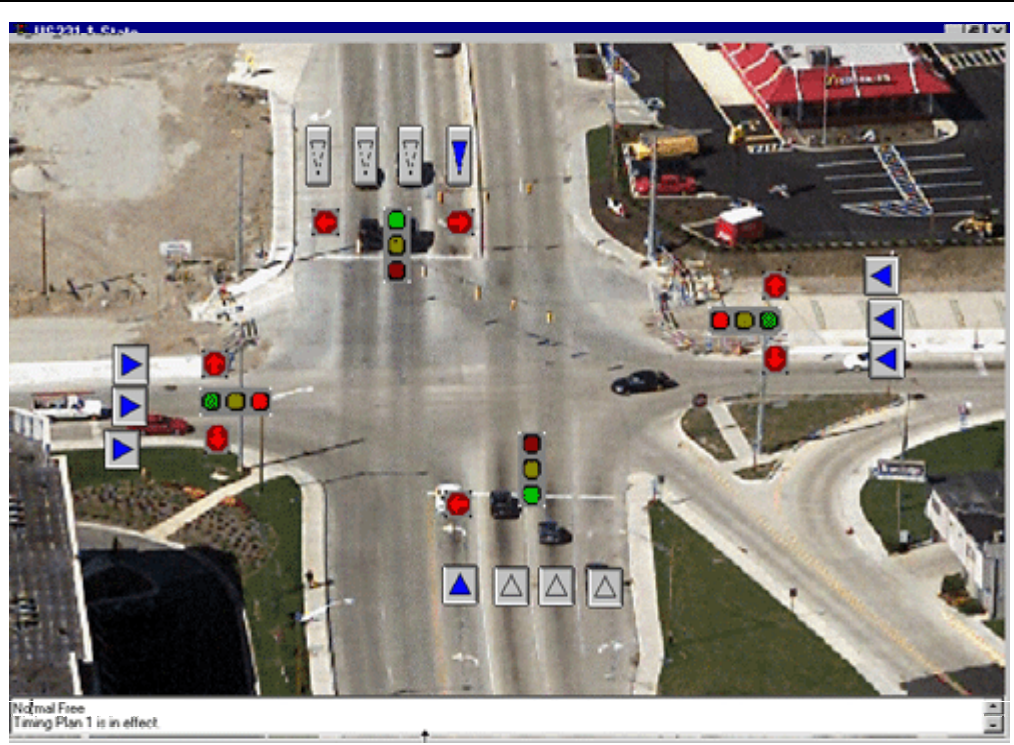

Figure 4-62 CLMats Graphics 


\subsubsection{Greenband Analysis}

The greenband analysis provides the user with a time-space diagram like the one shown in Figure 3-55. This allows the user to see the progression of vehicles through the system and makes it easier to visualize the effects of offsets. In order for the greenband analysis to work, the intersection links must be added. This is typically done after the intersections are added in Section 4.4.2.1. When the greenband analysis is launched, the display will not appear until the data for the display is collected. This usually lasts for a few controller cycles. The offsets can also be adjusted on the screen by clicking on the arrows in the display. The changes made in the offset here do not affect the offset in the controller. It is for testing purposes only.

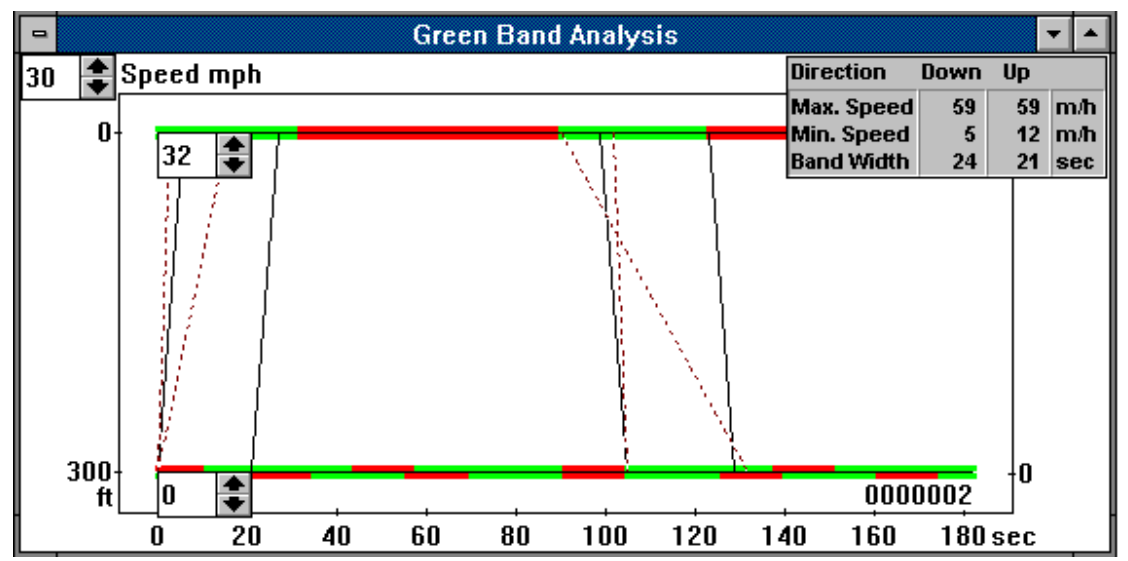

Figure 4-63 CLMats Greenband Analysis 


\title{
Chapter 5. Hardware-in-the-loop Simulation for Testing Controllers
}

\begin{abstract}
As was discussed in Chapter 1, it is very important to test the configuration of the controller and the timing parameters in lab setting. The parameters that were designed in Chapter 2 were based on general practices and standards. They need to be tested under simulated traffic conditions to ascertain their effectiveness. Testing the parameters using a hardware-in-the-loop simulation also serves as a check to ensure that the controller is configured correctly. After the configuration has been checked in the lab environment, the system can be implemented in the field and tuned. Field tuning procedures are outlined in Chapter 6.
\end{abstract}




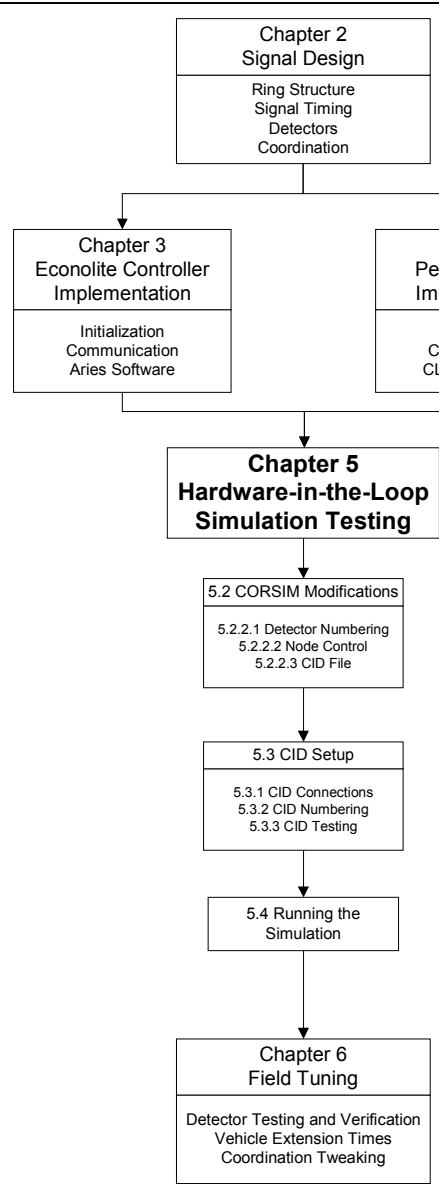

Figure 5-1 Chapter Concepts 


\subsection{Background}

Traditionally, the verification process in the lab has been to use a suitcase tester like the one shown in Figure 5-2a. The suitcase tester shows the phase indications and allows the user to place detector calls on phases. An alternative to the suitcase tester is to use a controller interface device (CID) shown in Figure 5-2b. The CID provides an interface between a controller and a computer. Various computer software programs can be used to test detector inputs and to monitor phase indications. The CID can also be used in conjunction with CORSIM to perform a hardware-in-the-loop simulation, where the simulation software and the controller interact in real time.

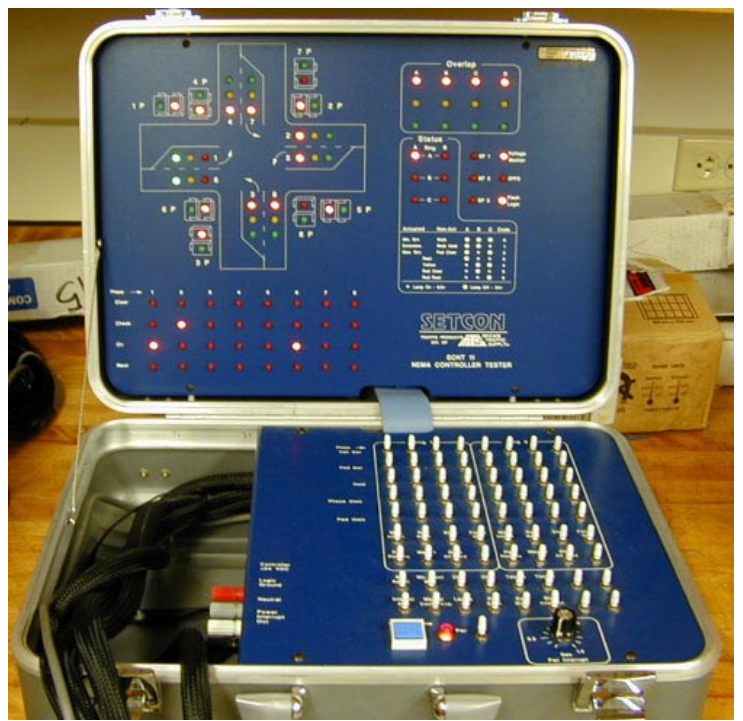

(a) Suitcase Tester

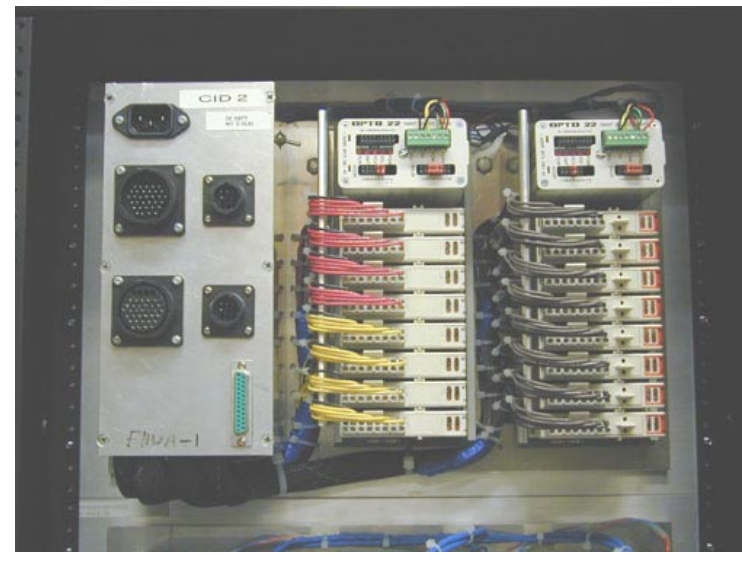

(b) Controller Interface Device (CID)

Figure 5-2 Controller Testers

\subsubsection{Suitcase Testers}

Suitcase testers provide a very quick and simple method of testing controllers. The LEDs show what phases, overlaps, and detectors are active. The detectors can be turned on and off, as well as other parameters, such as max times and force offs. Other features are also available, depending on how old the tester is. The major limitation of the traditional suitcase tester is the inability to simulate real traffic scenarios. It is very difficult to keep turning detectors on and off to emulate traffic, while watching the phase timers and transitions. There is no way to get a good picture of how the controller would perform under actual traffic conditions. With a CID and real- 
time simulation, all of the detectors can be tested and simulated traffic can be used to assess the controller performance.

\subsubsection{CID Testers}

The CID is interfaced to the controllers' A, B, and C connectors. The interface to the computer is through a serial cable. Figure 5-3 depicts the architecture of a hardware-in-the-loop simulation. There are six basic components of the setup. Items A, B, and C represent the controller side of the setup and items $D, E$, and $F$ represent the simulation side of the setup.

(A) The local controllers are used to control the intersections in the simulation. The local controller receives the vehicle calls and generates the phase states. One local controller is needed for each intersection in the simulation.

(B) The master controller communicates with the local controllers to set their time clocks and monitor other activities. The master also provides an interface for the central computer to monitor all of the local controllers with a single connection.

(C) The central computer running Aries or CLMats is used to monitor the system. The monitoring of local controllers is typically done through the master controller.

(D) A CID provides the interface from the local controller to the computer running a simulation model. The CID monitors the controller phase states and the simulation detector states.

(E) The simulation software is responsible for moving vehicles through a defined network and tabulating output data. The simulation does not implement any control logic. It uses the signal states obtained from the CID and sends the detector calls to the CID for the controller to use.

(F) A software interface module provides the linkage between the CID and the simulation program.

Since standard signal controllers are required to have the same configuration for detector inputs and phase outputs, the real-time simulation with a CID is compatible with a variety of controllers. 


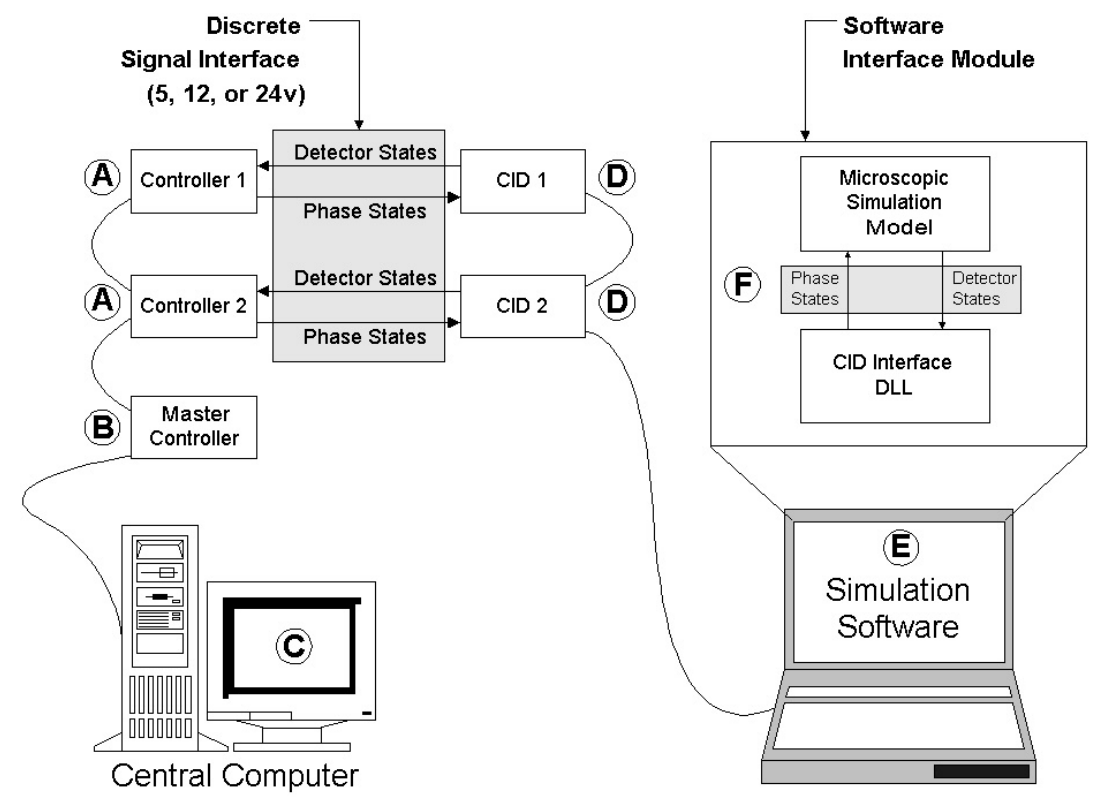

Figure 5-3 Hardware-in-the-Loop Architecture

The layout of the CID is shown in Figure 5-4.

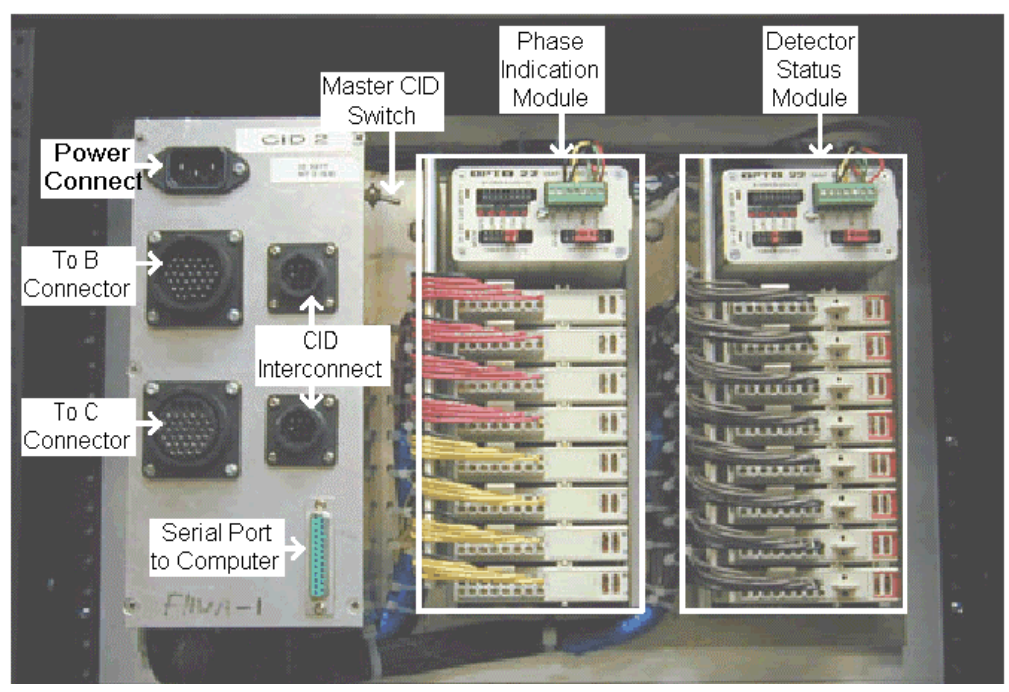

Figure 5-4 CID Layout 


\subsection{Simulation Modifications}

\subsubsection{Simulation Specifics}

Since the simulation is being controlled externally, the only data that is important in the simulation file is the detector information and the volume information. If a simulation file has to be created for the hardware-in-the-loop simulation, not all parameters need to be configured for the system. The nodes and link locations are important, as well as the entry volumes and turning statistics. The nodes should be set up as actuated controllers. The phases should be assigned to their corresponding turning movements. The proper detectors also need to be configured. If a simulation file already exists, only a few modifications need to be made for it to be used for a hardware-in-the-loop simulation.

\subsubsection{Simulation File Modifications}

\subsubsection{Detector Numbering}

Two different types of detectors can be configured in ITRAF. They are detectors for actuated control in record 46 and surveillance detectors in record 42. The surveillance detectors are the ones used in the hardware-in-the-loop simulation. The actuated control detectors are configured in the node control screens. Having these detectors configured will not affect the hardware-inthe-loop simulation. The surveillance detectors are configured in the link edit screens shown in Figure 5-5. Figure 5-5a shows the screen in the CORSIM editor and Figure 5-5b shows the simulation text file.

The surveillance detectors can be placed very precisely and set as presence or pulse. The detectors can also be assigned four digit numbers. These numbers shall correspond to the node number and the detector number in the controller. The first two digits indicate the number of the upstream node that is controlled by the same controller as the current detector. The last two digits of the number are the number of the detector in the controller. In Figure 5-5, this link detector is a 36 foot long presence detector at node 1 that is detector number 2 . In the controller that is linked to node 1 , detector number 2 would be enabled and assigned to phase 2 . 


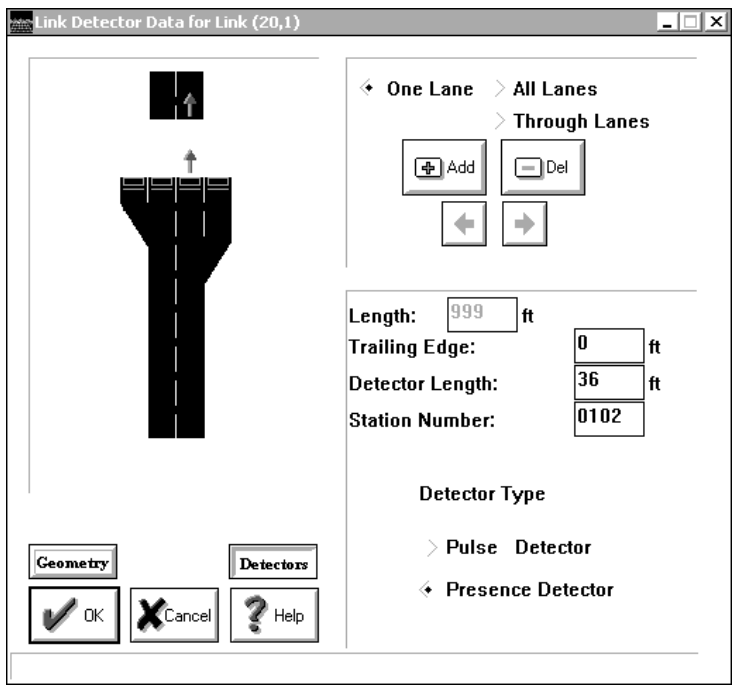

(a) CORSIM Screen

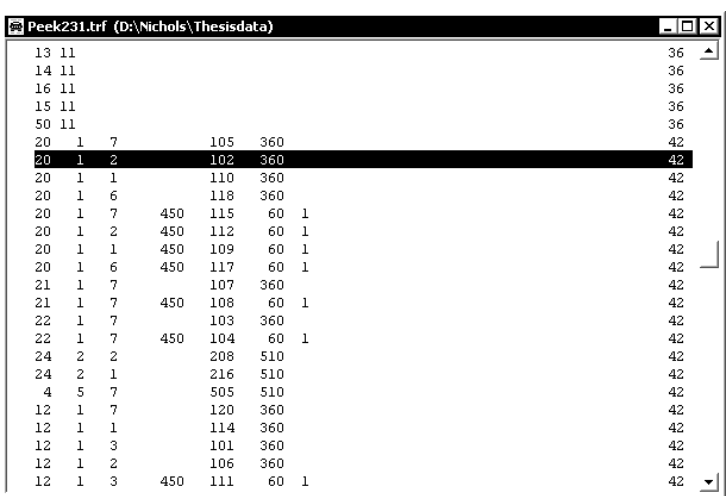

(b) Text File

Figure 5-5 CID Detector Configuration

\subsubsection{Node Control}

In record 43, placing a 2 in column 77 , as opposed to nothing, will flag this node for external control. Here external control refers to the controller. Without this number here, CORSIM will not know to look for an external controller and will perform a standard simulation.

\subsubsection{CID File Generation}

When CORSIM looks for the external controller for a node, it opens a file with extension ".CID" and the same root name as the "TRF" file. One CID file shall be created for each simulation. The template for the CID file is shown in Figure 5-6. The first lines of the file are used to assign the nodes to CIDs. First, the CIDs are assigned numbers, and then the nodes are assigned to the CID numbers. Next, the approaches have to be mapped to the proper phase numbers. The approach numbers can be found in the simulation file in record type 43. The first number in the column is the node number. The second and third numbers are the link that is approach 1 . The fourth and fifth numbers are approach 2, and so on. Once the approaches are numbered, the movements are assigned to the phase numbers. If the movement is powered by an overlap, the overlaps are phases $9,10,11$, and 12 for A, B, C, and D. Finally, the movement is designed protected, permitted, or both. All movements will be assigned as protected. The only exception is a left-turn that can be any of the three. 


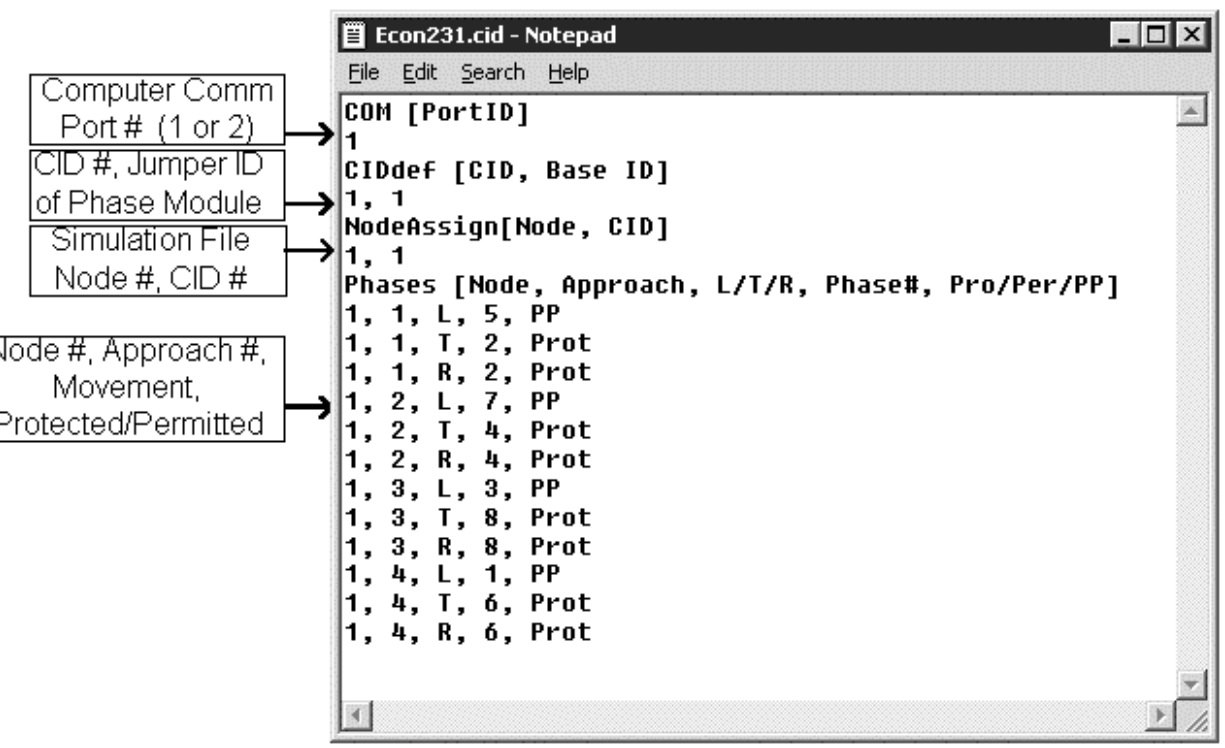

Figure 5-6 CID File Template 


\subsection{Controller Interface Device Setup}

\subsubsection{CID Connections}

One CID is required for every controller in the system. The CIDs should be connected to their controllers with the proper cables and plugged into a power supply. The CIDs must be interconnected with the appropriate 9-pin cable. There are two 9-pin ports on the front of the CID. It does not matter which port is used because they function similarly. All of the CIDs should be daisy-chained as shown in Figure 5-7. Once the CIDs are interconnected, one has to be connected to the simulation computer with a serial cable. On this CID, the toggle switch on the inside of the panel must be UP. The toggle switch on the other CIDs must be in the DOWN position. This denotes the CID that controls the communication between the computer and the rest of the CIDs.

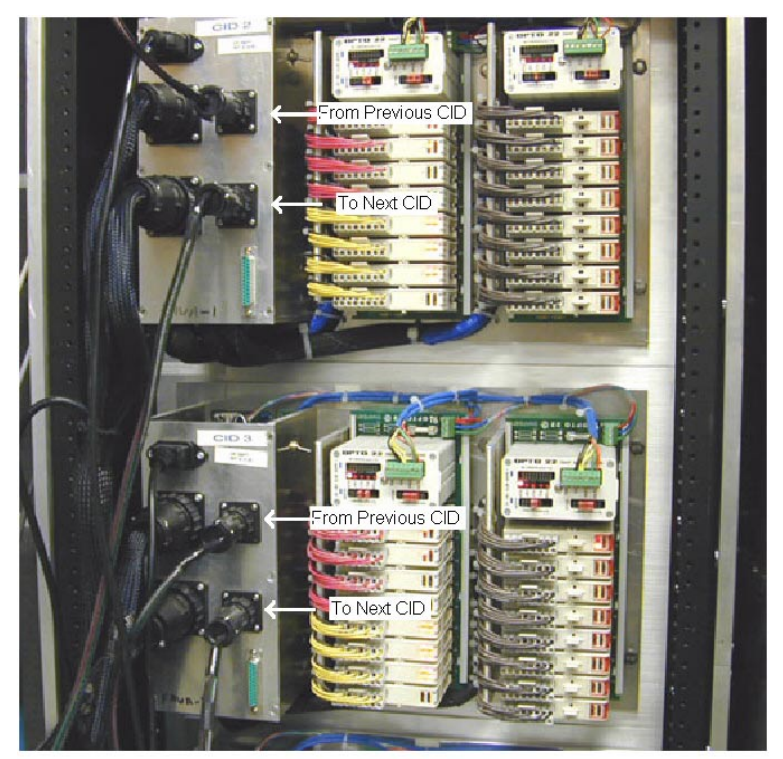

Figure 5-7 CID Interconnect

\subsubsection{CID Numbering}

The CIDs must all have a unique identification. In the CID file, the CIDs were numbered for each node. Each CID has two modules - one for the detectors and one for the phases. On CID 1, the phase module has ID 1 and the detector module has ID 2. On CID 2, the phase module has ID 3 and the detector module has ID 4. This numbering scheme is used for all of the CIDs in the system. These numbers should correspond to the numbers assigned in the CID file. The module numbers are configured on each module as shown in Figure 5-8. Jumpers must be placed on the 
prongs to form the addresses. The numbering of the prongs is binary from right to left $(1,2,4,8$, 16,32 ). To form address 5 for the phase module of $\operatorname{CID} 3$, a jumper would be placed on the rightmost prong and on the third prong from the right end for 1 and 4. Table 5-1 shows the jumper configuration for five CIDs. Likewise, the detector module would have jumpers on the second and third prongs from the right end to form address 6 .

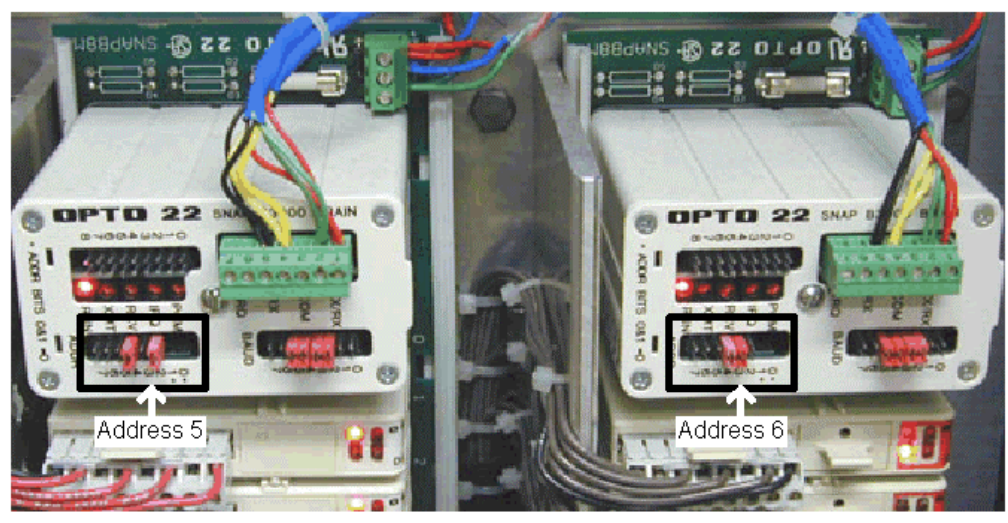

Figure 5-8 CID Module Jumpers

\section{Table 5-1 CID Numbering}

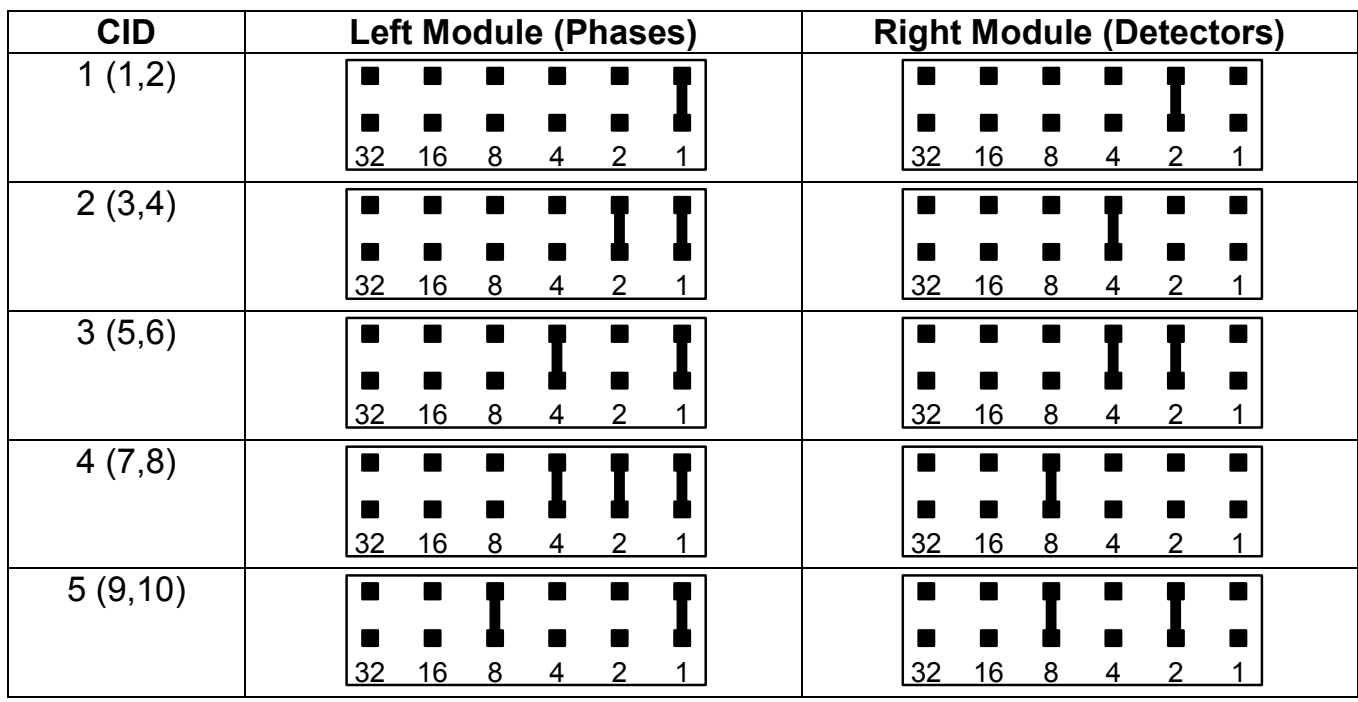

\subsubsection{CID Testing}

Once the CIDs have been connected to the controllers, interconnected, and numbered, they need to be tested. The phases can be confirmed by looking at the controller status display and the phase indications on the phase module. The green phase indications show up on the top 3 
modules in the phase indication module. If a phase is green, the LED corresponding to that phase will be on. A utility known as intsect2 has been developed to test the detectors. When the utility begins, the comm. port number must be assigned, as well as the phase module and detector module numbers for the desired CID. If the software connects to the CID, the screen shown in Figure 5-9 will appear. Detectors can be turned on and off by clicking in the boxes. The numbering scheme for the detectors in this window correspond to the numbering on the detector module, with 1 being in the top left corner and the numbers increasing vertically, then horizontally. The detectors should not only be confirmed on the CIDs, but also in the controller. This is easily done in a detector status screen that shows each individual detector input. All CIDs in the system should be tested. If the software cannot connect to a CID, check to make sure the address is correct and it does not conflict with any other addresses. Also check the toggle switches and the interconnect cables.

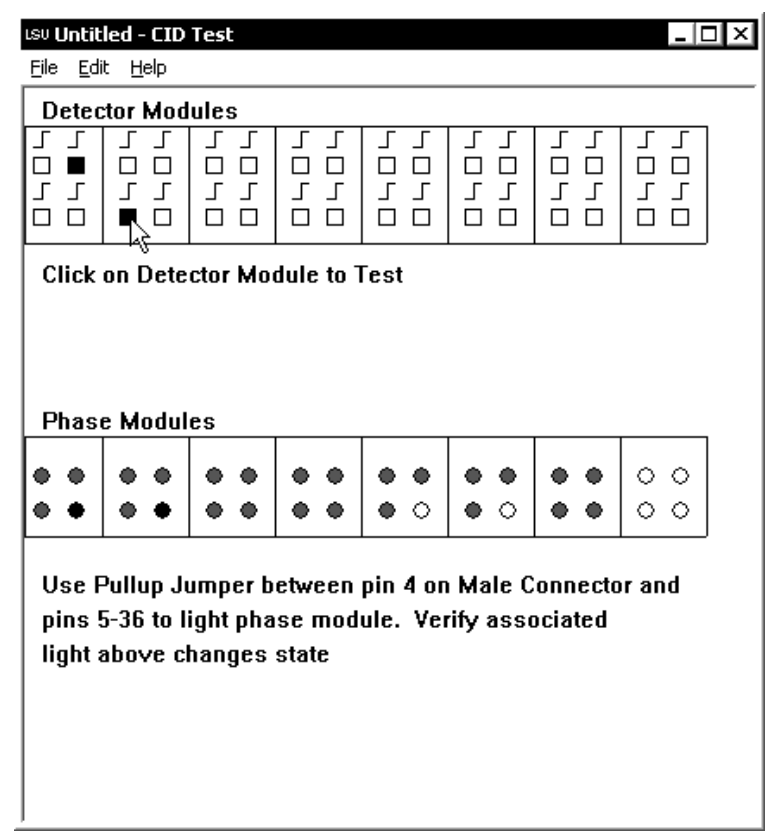

Figure 5-9 CID Testing Utility 


\subsection{Running the Simulation}

After all of the CIDs have been connected and the simulation files modified, the simulation can be executed. The simulation can only be run with the RTContrl plug in. This file, along with RTTrafvu to view the simulation can be installed onto the computer. Once the simulation begins, the CID file is opened up and the phases are assigned to the CIDs. It is important to note that since the simulation is in real-time, it takes one minute on a clock to perform one minute of simulation. This is very important while the simulation is running. CORSIM displays the computer time that it took to perform one minute of simulation. The computer time should be exactly one minute. If it takes the computer longer to run one minute than the controller, the simulation is not valid. For example, if a 60 second simulation moves vehicles over an 85 second period, the controller could give more green or red time to particular phases, leading to erroneous measures of efficiency. Figure 5-10a shows ideal scenario with the computer time matching the simulation time. Figure 5-10b shows an undesirable scenario with the computer time varying. Other programs running on the computer at the same time generally causes this.

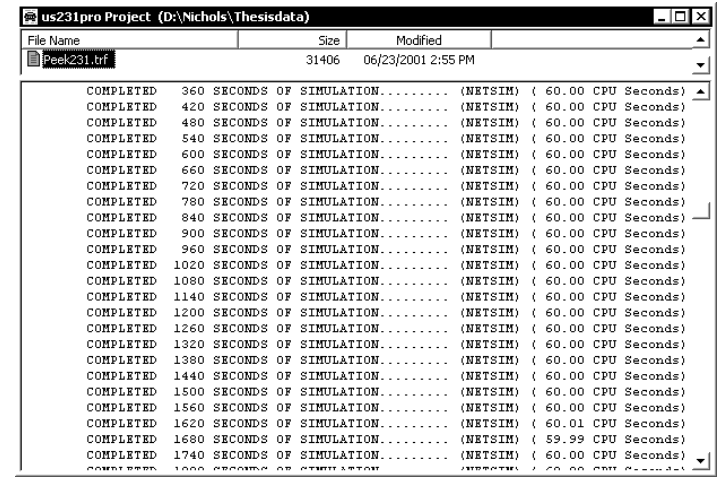

(a) Ideal Simulation

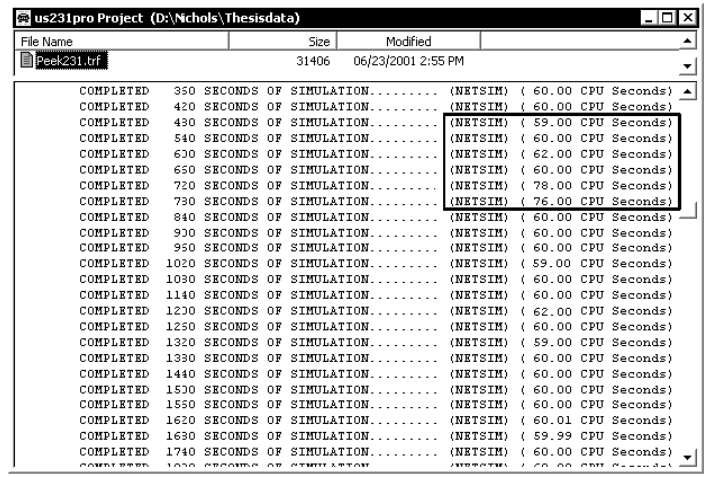

(b) Invalid Simulation

Figure 5-10 Simulation Time Periods

After the initialization period has ended and the simulation is running, it can be opened in RTTrafvu. Again, the simulation is being viewed in real time, so the animation will be somewhat sluggish.

After the simulation is finished, the output file from the simulation can be viewed and processed. This data can be used to produce very helpful tables and graphs that provide insight about how the system is performing. 


\section{Chapter 6. Field Tuning Closed Loop Systems}

After a closed loop system has been implemented in the field, it is very important to monitor the system performance and continually update the programs. Traffic patterns constantly change due to economic development and other, subtler factors. Citizens usually report serious problems in a system before a technician discovers them because citizens use the system every day. However, technicians should examine issues related to the efficiency of the system at least once a year.

The field tuning practices discussed in this chapter cover both immediate checks that deal primarily with safety and functionality and future checks that affect the efficiency and performance of the system. Immediately after the system is implemented, the detectors should be tested to make sure that they are working and are mapped properly. Errors in detector configuration and mapping are one of the main implementation problems, due to the fact that they are not easy to test. After the detectors are tested, the timing parameters should be validated to make sure they are not too short. Parameters that are too short can have safety implications, whereas parameters that are too long typically only decrease system efficiency. Finally, the coordination in the system should be checked on a yearly basis. This check is very subjective and imprecise because the coordination will never be perfect. Problems at one intersection in the system can affect the progression through the rest of the system. Fixing the problem can be very complex and have other implications. The fine-tuning of the system is an ongoing process. 


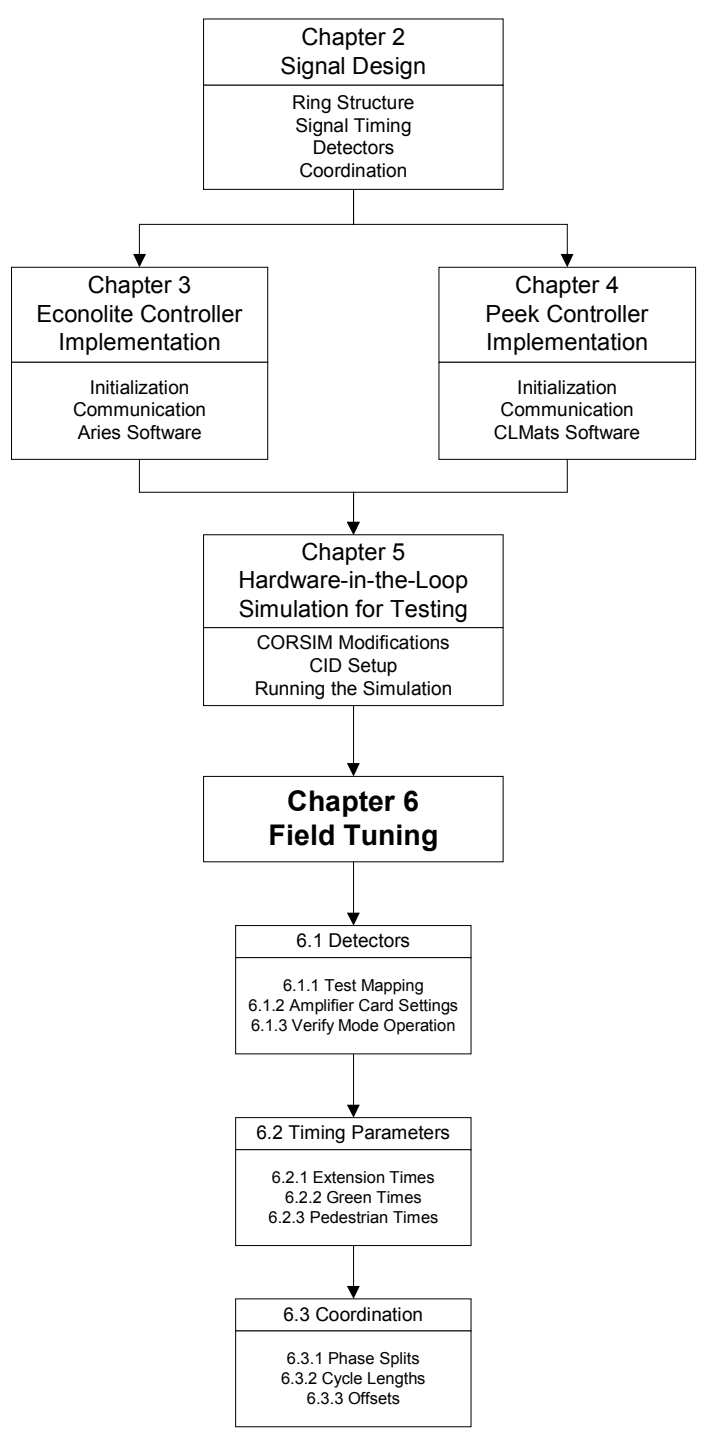

Figure 6-1 Chapter Concepts 


\subsection{Detectors}

\subsubsection{Detector Mapping}

Each intersection should be tested to ensure that all detectors are calling their proper phases.

This requires a map depicting which detectors are assigned to which lanes. It is recommended that the testing be done during low volume conditions because fewer detectors are active simultaneously. Loops can be triggered either by a second person driving a vehicle or by dragging a 2-foot in diameter copper wire loop like the one shown in Figure 6-2a. The status display screen will allow the technician to see which phases are being called when a detector is active. However, in order to determine if the detector is mapped to the correct lane, the detector input display in Figure 6-2b must be used. This is important when detectors are configured to extend/delay or count. Knowing which lanes the counts are for is very important, especially for turning lanes.

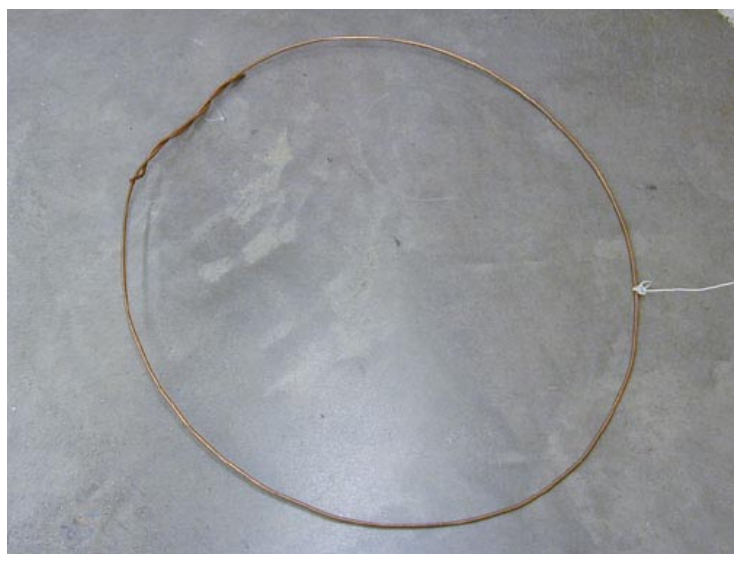

(a) Copper Loop

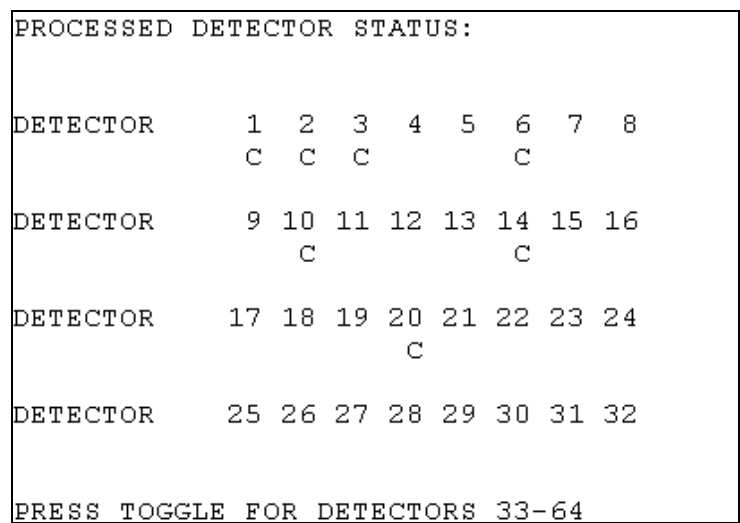

(b) Detector Input Display (MM, 7, 6)

Figure 6-2 Detector Field Testing

If the detectors are not mapped correctly, the easiest and quickest way to fix the problem is in the controller. Determine the channel of the detector that is wrong and reassign it to the correct phase. This procedure is outlined in Section 3.4.4.4.1 for Econolite controllers and Section 4.4.4.4 for Peek controllers. 


\subsubsection{Amplifier Card Settings}

The detector amplifier cards in the cabinet should be checked to make sure the settings are correct. They should be configured for presence mode. The sensitivity should also be checked. The sensitivity adjusts how far from the center of the loop a vehicle is still detected. This will have to be adjusted to ensure that vehicles in an adjacent lane do not activate the detector.

\subsubsection{Detector Modes}

Make sure that any detector features that are enabled in the controller are working properly. These include extend and delay times and detector logging. Check to make sure that data is being logged either by viewing the screens in the controller or by downloading the data to a computer. 


\subsection{Timing Parameters}

\subsubsection{Vehicle Extension Times}

Verify that vehicle extension times are not too long or too short, particularly in the left-turn lanes. Long extension times lead to decreased capacity because of the amount of time not used by a vehicle at the intersection. It is wasted while the phase is waiting on a vehicle to arrive that may not arrive. Short extension times can cause a phase to gap out too early for trucks and other slow moving vehicles because they have a longer startup time. If a truck is 5 or 6 vehicles back from the stopbar, the vehicles in front of it that can accelerate quicker will all extend the green and pass through the intersection. However, if the extension time is too short, the phase may gap out before the truck can reach the detector.

\subsubsection{Green Times}

From a safety standpoint, the minimum greens should be checked to make sure they are not too short. This is very important on high-speed approaches that have detection because approaches without detection are in recall. Also, the maximum green times should be checked while the intersection is in free operation since the maximum times are inhibited during coordination. If the green times were calculated based on the guidelines in Section 2.2.4, they should be acceptable.

\subsubsection{Pedestrian Times}

The pedestrian walk and clearance times should be adequate for the pedestrian movements. They should not be too short because that will affect the safety of the intersection. If they are too long, that will only decrease the efficiency. Check to make sure the times were calculated in accordance with Sections 2.2.3 and 2.2.3.2. 


\subsection{Coordination}

\subsubsection{Phase Splits}

Verify that all phase splits are long enough. If splits are not long enough, excessive queuing may occur on particular movements. If one split is increased, another split has to be decreased because the cycle length cannot change.

\subsubsection{Cycle Length}

If the intersection is running under saturated conditions on multiple approaches, the cycle length may need to be increased. This decision should not be made without prior planning due to the fact that this modification will affect the entire system. All intersections have to run a common cycle length for coordination. The intersection could be double-cycled to maintain coordination, but a new offset will have to be obtained with optimization software.

\subsubsection{Offsets}

Verify that offsets are appropriate by driving the system. Generally, one cannot expect perfect progression in both directions as shown in Figure 2-18a. Consequently, the system offsets are typically tweaked to provide good progression in one direction and moderate progression in the other - similar to that shown in Figure 2-18b. 


\section{Chapter 7. Conclusions}

Chapter 2 established the procedure for designing the different areas of the traffic signal control for a system. Intersection-specific parameters were discussed and calculated that included ring structures, phases, timing data, detector data, and coordination. By following these standards and recommendations, traffic signal control will be much safer with less parameters being configured without any support.

The parameters calculated in Chapter 2 were incorporated into the system in Chapter 3 and Chapter 4. These chapters outlined the configuration of Econolite and Peek traffic signal controllers for a closed loop system. The primary steps included controller initialization, communication between the controllers, data entry, and remote monitoring software. Traffic signal controllers have many menus with many parameters to configure. It is very easy to forget about a menu or leaving out something. If followed correctly, these steps will ensure proper configuration of a successful system.

Chapter 5 introduced the procedure for testing the controllers after they were configured. The procedure involved using a hardware-in-the-loop simulation in a lab for testing the controllers in real-time. The key component that makes the simulation possible, a controller interface device (CID), was discussed in detail, along with how to set up the simulation. By testing the controllers in a lab setting before they are deployed in the field, many problems can be avoided that may possibly jeopardize the safety of the public.

Chapter 6 covered the final step in the deployment process - tuning the system after it is in the field. Procedures and checks were provided for the detectors, timing parameters, and coordination plans. It is essential to fine tune the system after it has been deployed. Traffic 
patterns constantly change and the signal systems must adapt to these changes to remain effective.

By following the procedure outlined in this manual, the process of deploying closed loop systems will be greatly facilitated. If deployed correctly, the closed loop systems will increase the performance of traffic signal control and ultimately create a safer, more reliable network of highways. 


\section{Appendix A. Glossary}

AB3418 - communication protocol used in California.

Actuation - placing a call to the traffic signal controller by activating a loop detector. This call lets the controller know that a vehicle is present and where it is located.

Amplifier Card - installed in the detector rack in the signal cabinet for inductive loop detectors. The cards have 2 or 4 channels and convert the signal from the detector into a signal that the controller can understand. Typically, 2 channel cards are used in Indiana.

Barrier - in a ring structure, the barrier separates phases that cannot be on concurrently. Two phases that are located on different sides of a barrier will never be on at the same time.

Coordination - relationship between controllers in a closed loop system to provide progression through the system along the main street.

Extension - act of extending the green indication of a phase by activating a detector.

Fiber optic cable - high speed data cable that can be used to interconnect controllers in a closed loop system. The data rates can reach 19,200 bits per second with a fiber interconnect.

Inductance - electrical property of a circuit with units of Henries. 
Local Controller - provides traffic signal control at a single intersection, and sometimes multiple intersections such as diamond interchanges.

Master Controller - does not provide traffic signal control, only provides control of interconnected local controllers. The master is used to monitor a closed loop system when accessed from a remote computer running management software.

NEMA - (National Electrical Manufacturer's Association) writes standards for traffic signal controllers.

NEMA TS1 - original traffic controller standard released in 1989. Does not address coordination, preemption, or more use of more than eight detectors.

NEMA TS2 Type I - traffic control standard released in 1992 that addressed coordination, preemption, and multiple detectors using an SDLC communication bus.

NEMA TS2 Type 2 - traffic control standard released in 1992 that used the legacy 24volt interface of TS1 instead of the SDLC interface.

NIC - (Non-interconnect) refers to the time-of-day schedule in the Econolite local controller that calls coordination plans.

Overlap - can be programmed to allow a phase outside of the ring structure or another phase in the ring structure to be green concurrently with the programmed phase.

Phase - assigned to each vehicle and pedestrian movement at an intersection and makes up the ring structure.

Preemption - special sequence of phases and timings that allows a vehicle placing the preemption call (usually an emergency vehicle, bus, or train) to have priority in the intersection.

Recall Mode - places a call on a phase so that the minimum green or maximum green time is served regardless of the actual vehicle presence.

Ring Structure - the map of the sequence of phases.

Telemetry - communication between controllers in a closed loop system. 
TOD - (Time-of-Day) refers to the time-of-day schedule that calls the coordination plans

Zone - the Peek master controller, can control up to four zones, calling separate TOD plans for each zone. 
Appendix B. U.S. 231 System 


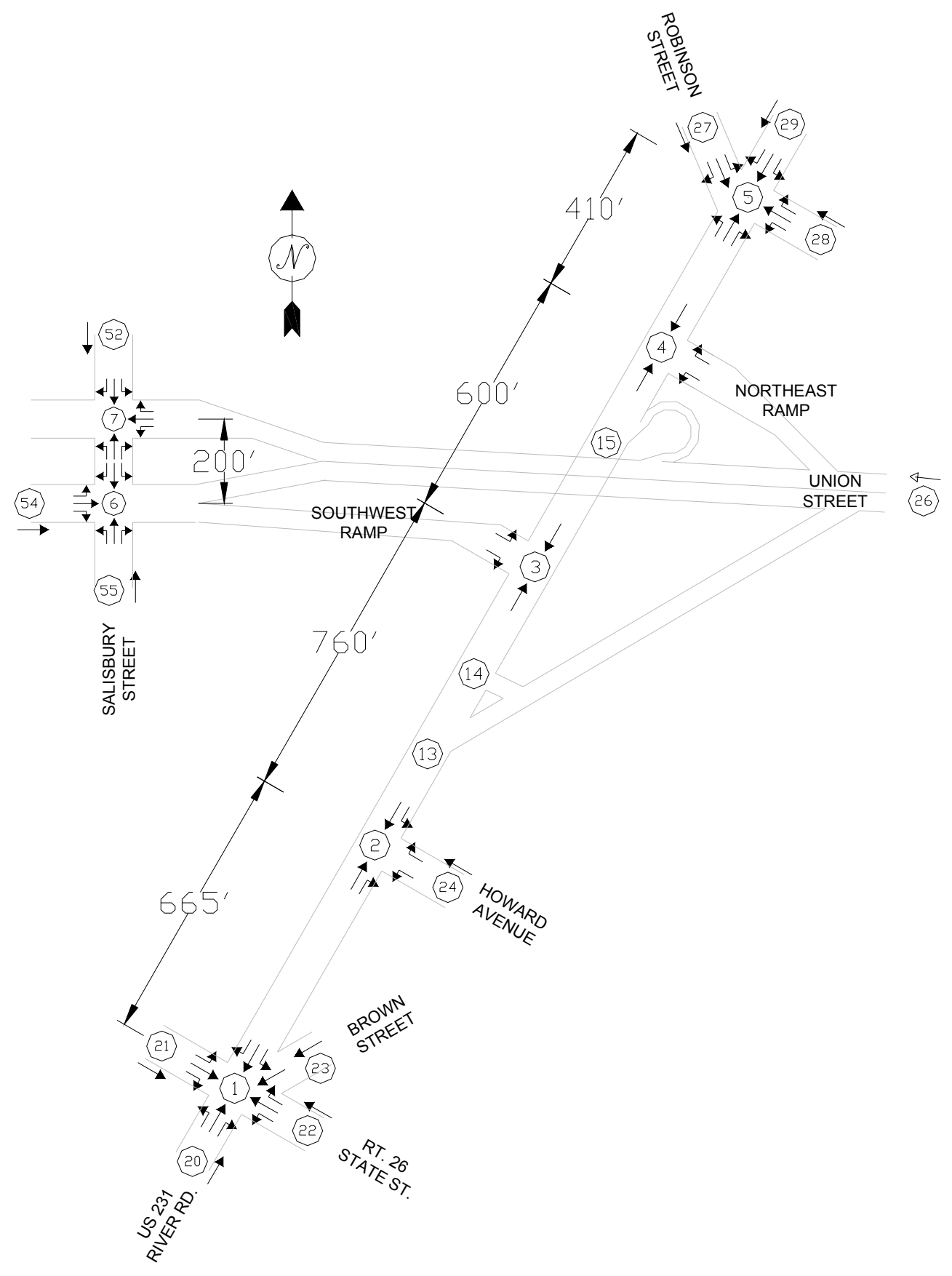

Figure B-1 US 231 (River Road) Network - State St. to Robinson St. 


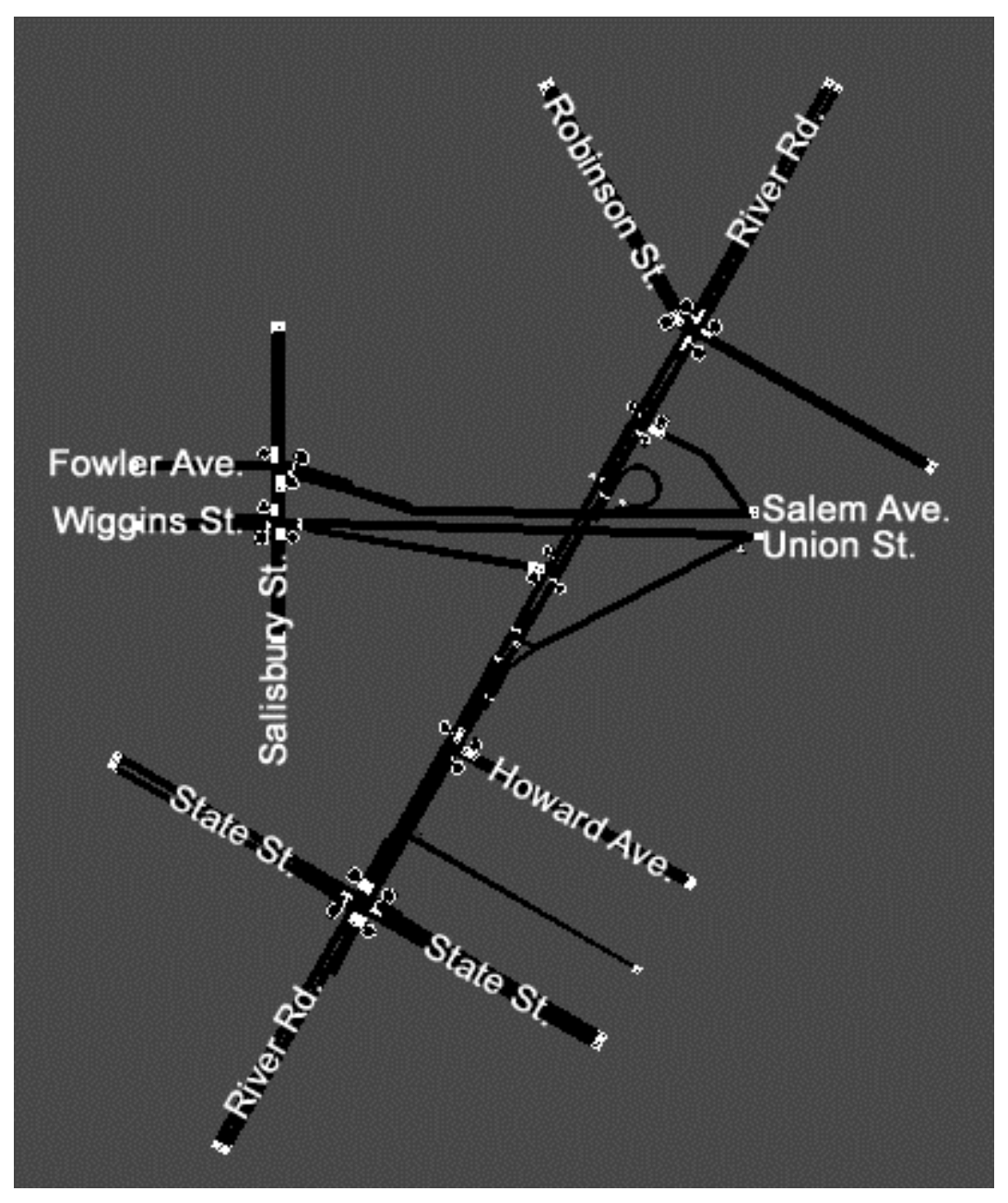

Figure B-2 US 231 (River Road) Network - State St. to Robinson St. (Screen Capture) 


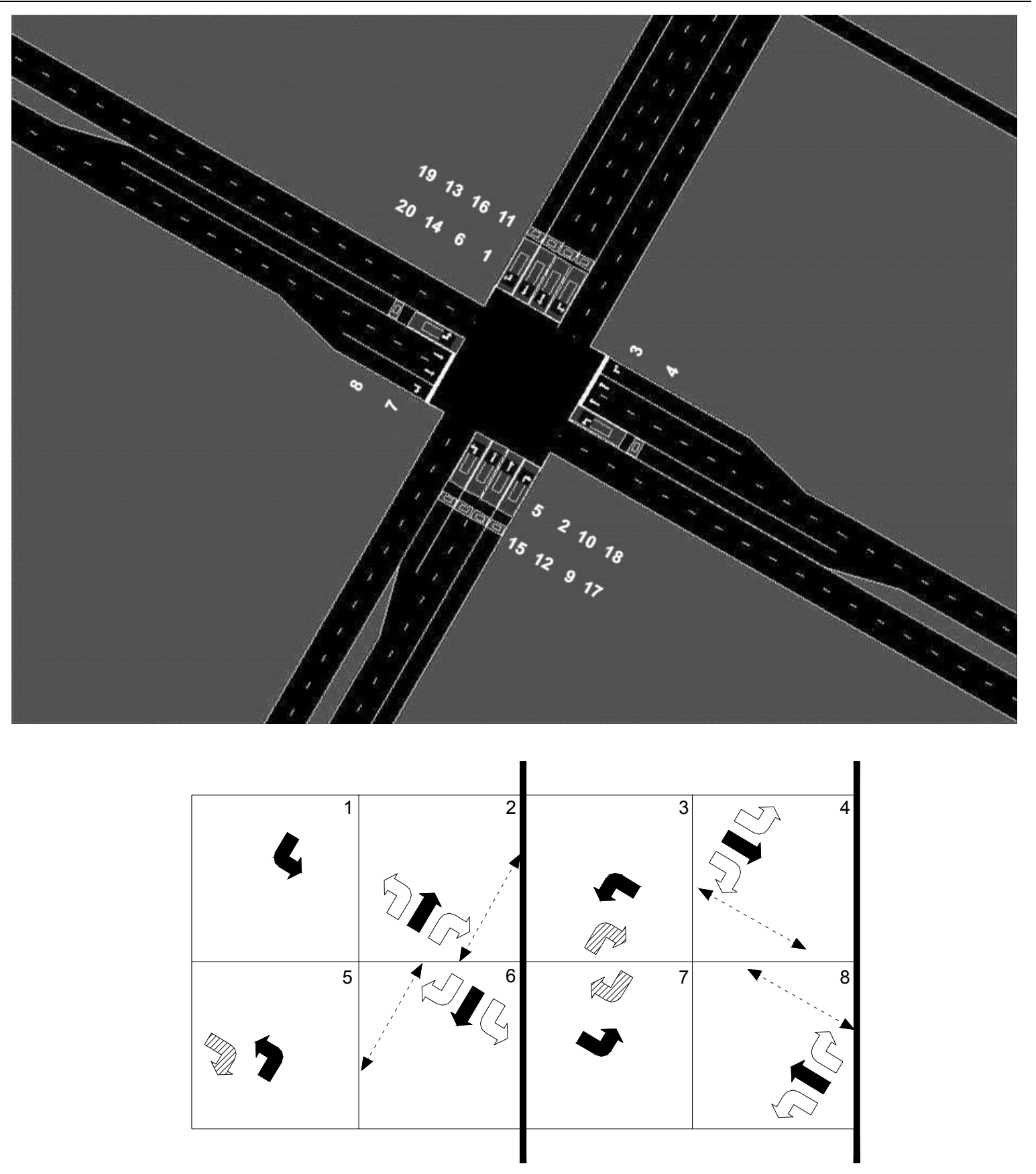

Figure B-3 Intersection 1: River Rd. \& State St. 


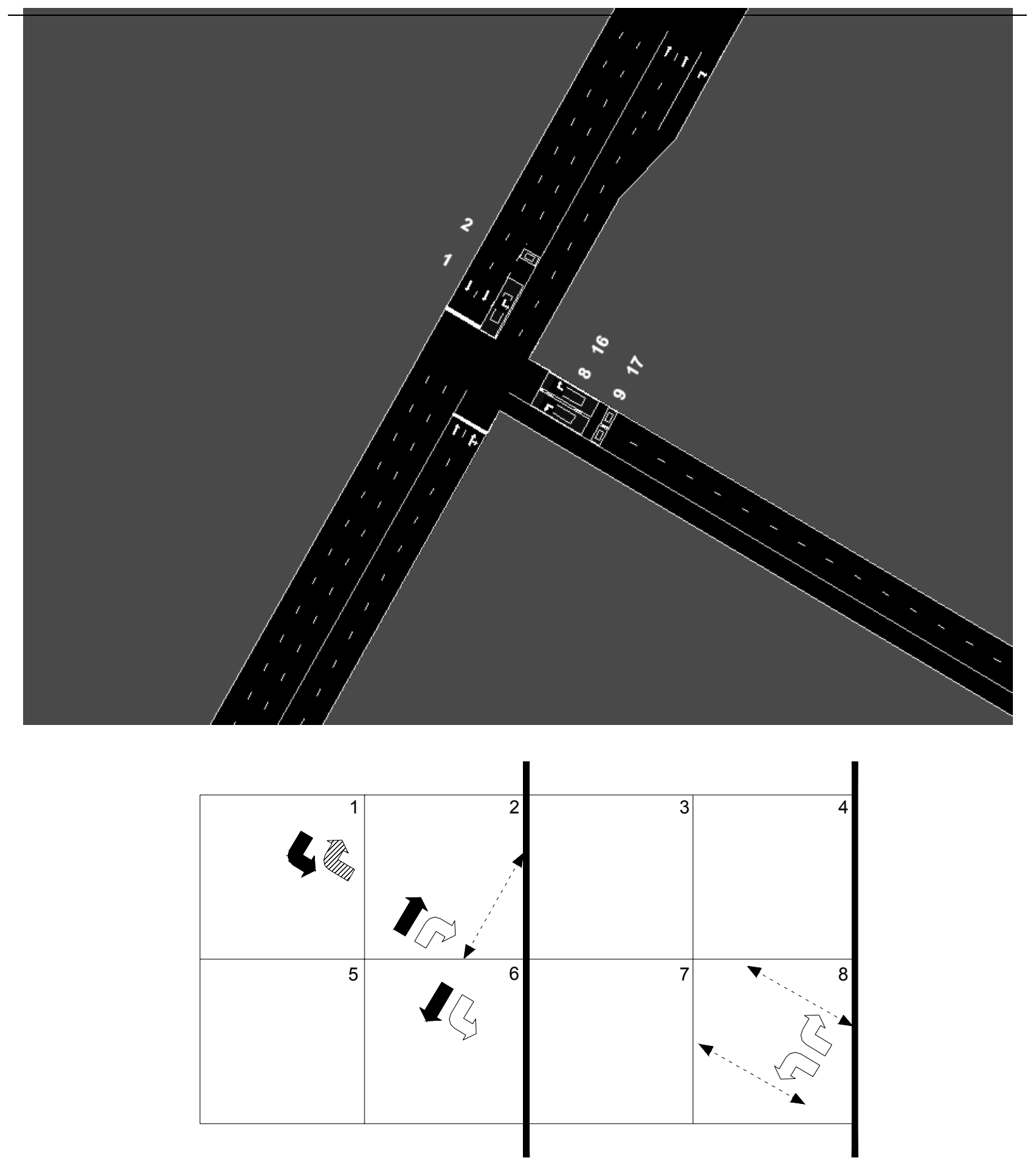

Figure B-4 Intersection 2: River Rd. \& Howard Ave. 

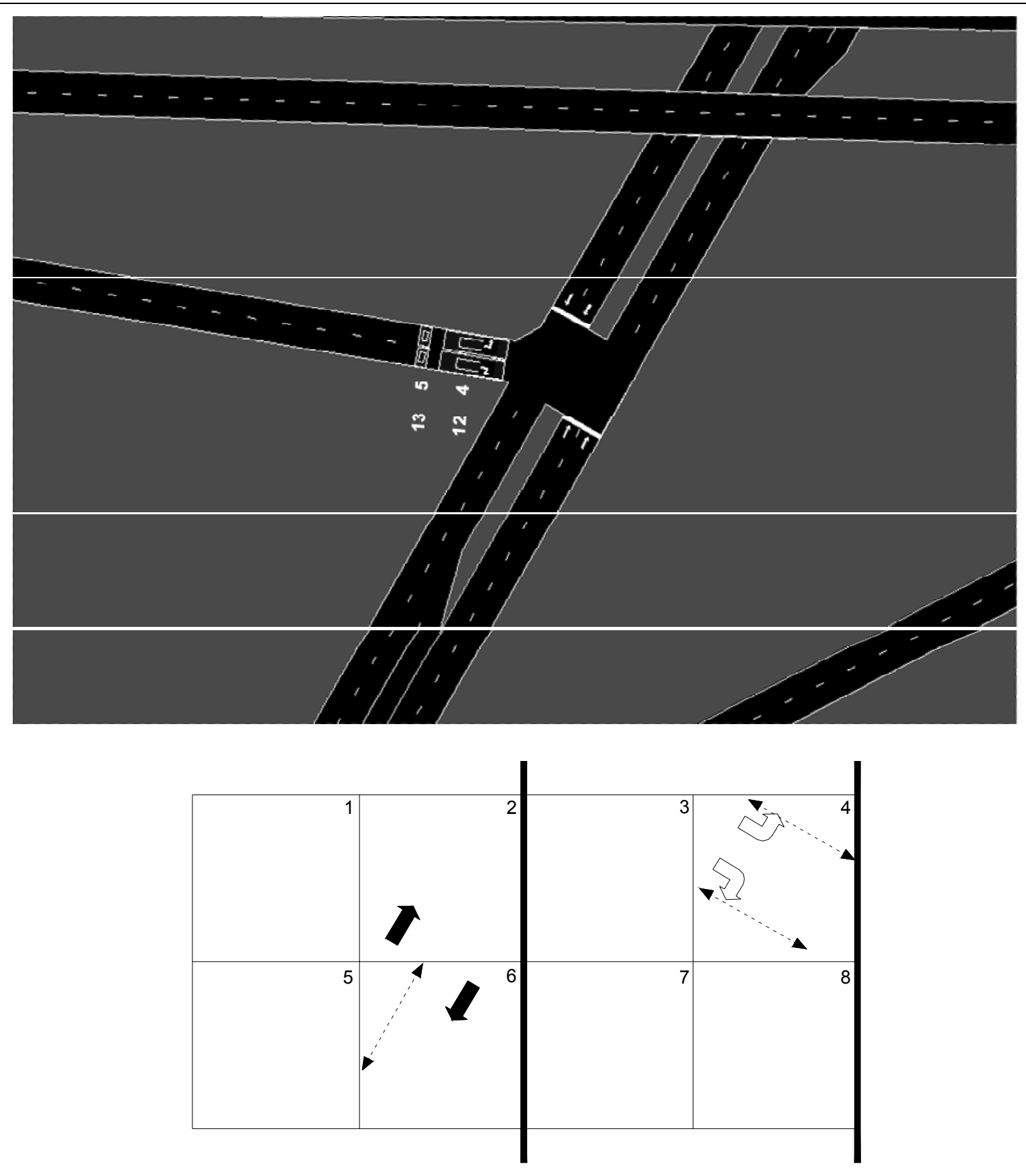

Figure B-5 Intersection 3: River Rd. \& Southwest Ramp 

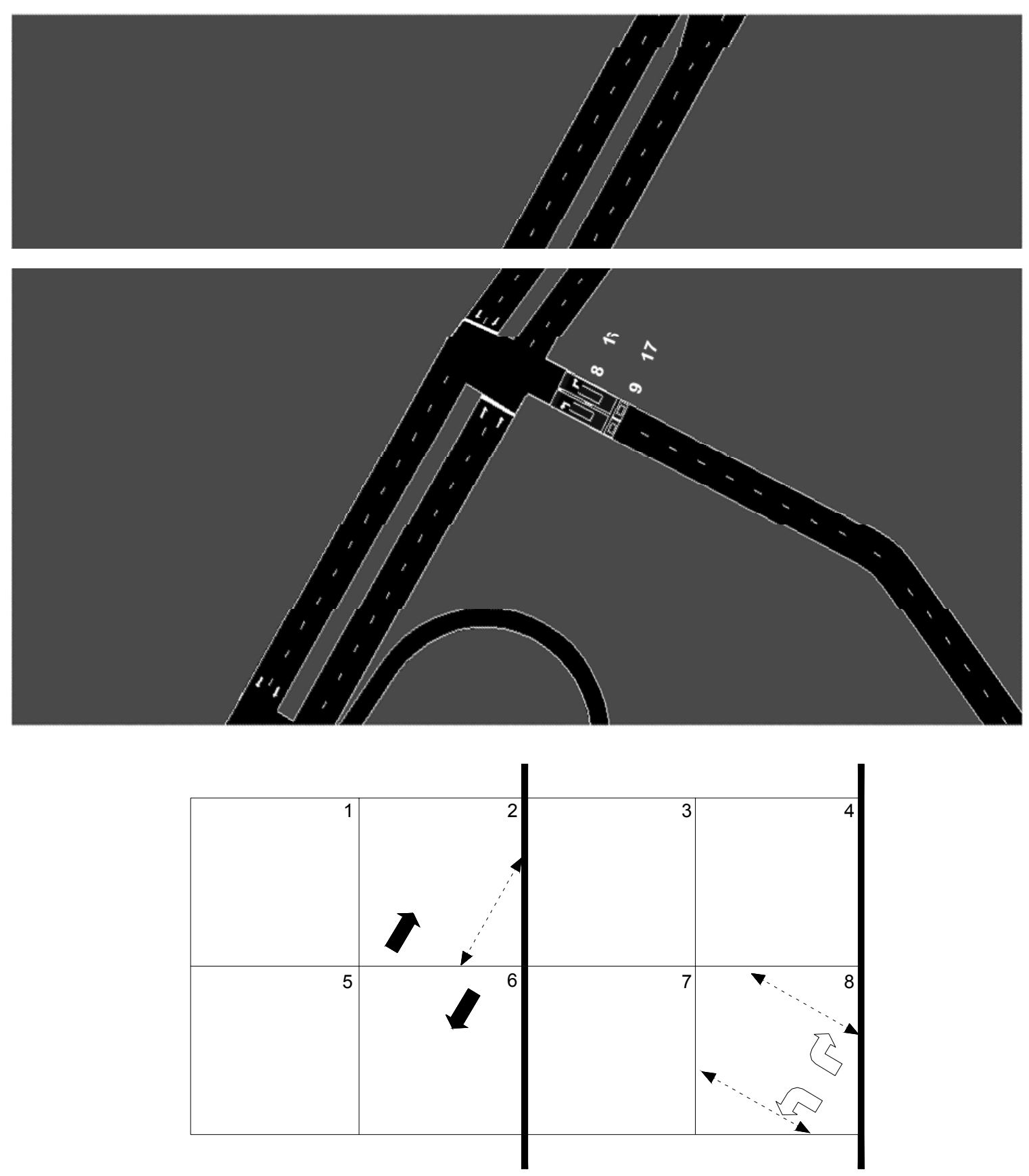

Figure B-6 Intersection 4: River Rd. \& NE Ramp 

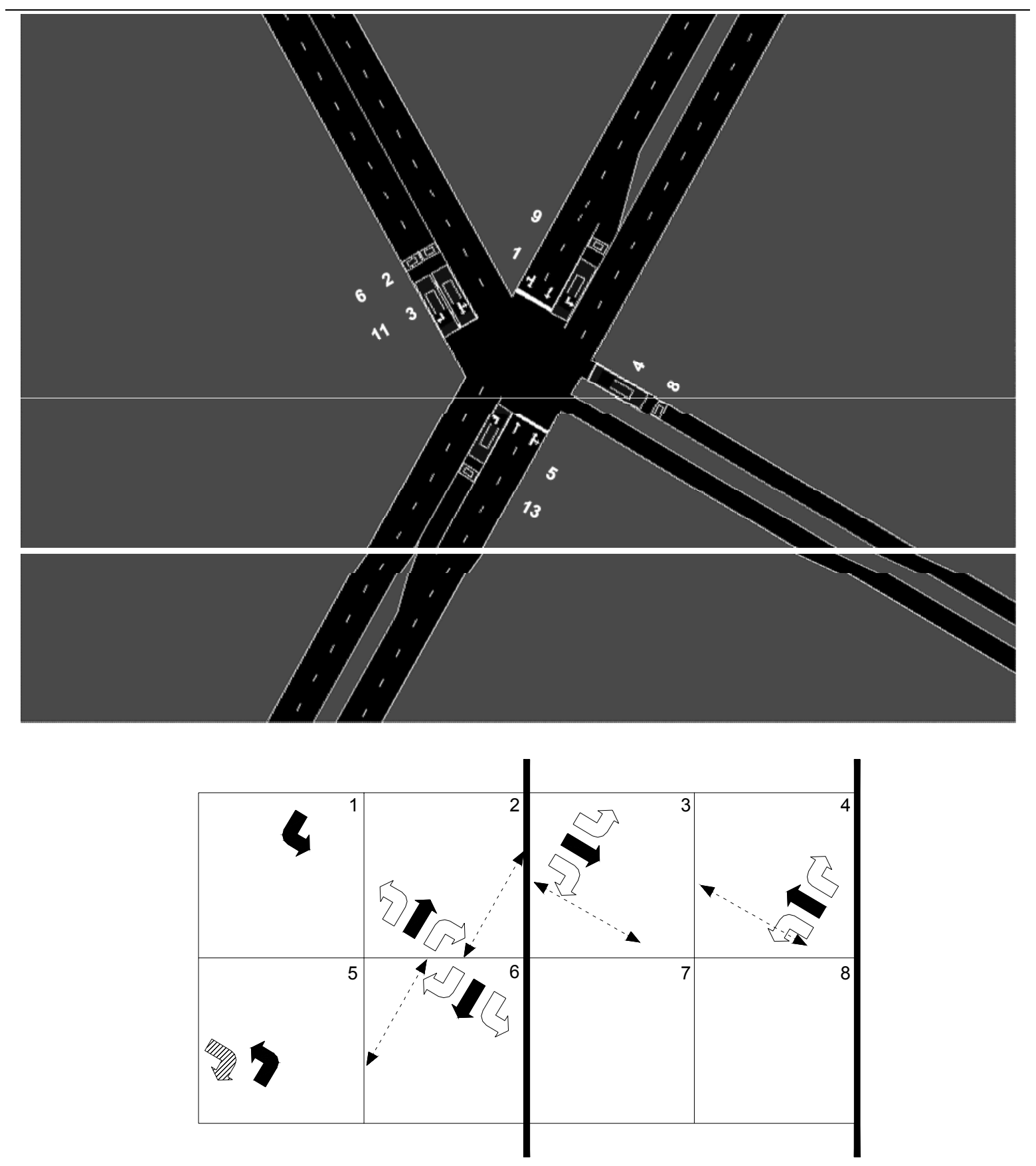

Figure B-7 Intersection 5: River Rd. \& Robinson St. 
Table B-1 System Entry Volumes

\begin{tabular}{|c|c|c|c|c|c|c|c|c|c|c|c|c|}
\hline ORIGIN & 8020 & 8021 & 8022 & 8023 & 8024 & 8026 & 8027 & 8028 & 8029 & 8052 & 8054 & 8055 \\
\hline MOVEMENT & NB & EB & WB & WB & WB & WB & EB & WB & SB & SB & WB & NB \\
\hline NODE & 1 & 1 & 1 & 12 & 2 & 4,7 & 5 & 5 & 5 & 7 & 6 & 6 \\
\hline PM PEAK & 800 & 1600 & 1300 & 0 & 488 & 1500 & 900 & 50 & 500 & 200 & 800 & 200 \\
\hline
\end{tabular}

Table B-2 System Turning Percentages

\begin{tabular}{|c|c|c|c|c|}
\hline NODE & APP & LEFT \% & THRU \% & RIGHT \% \\
\hline 1 & NB & 7 & 81 & 12 \\
\hline 1 & SB & 12 & 79 & 9 \\
\hline 1 & EB & 6 & 89 & 5 \\
\hline 1 & WB & 8 & 82 & 10 \\
\hline 2 & NB & -- & 80 & 20 \\
\hline 2 & SB & 25 & 75 & -- \\
\hline 2 & WB & 40 & -- & 60 \\
\hline 3 & NB & -- & 100 & -- \\
\hline 3 & SB & -- & 100 & -- \\
\hline 3 & EB & 50 & -- & 50 \\
\hline 4 & NB & -- & 100 & -- \\
\hline 4 & SB & -- & 100 & -- \\
\hline 4 & WB & 20 & -- & 80 \\
\hline 5 & NB & 15 & 80 & 5 \\
\hline 5 & SB & 2 & 83 & 15 \\
\hline 5 & EB & 35 & 5 & 60 \\
\hline 5 & WB & 40 & 40 & 20 \\
\hline 13 & NB & -- & 78 & 22 \\
\hline 14 & SB & 20 & 80 & -- \\
\hline 15 & NB & -- & 77 & 23 \\
\hline
\end{tabular}


Table B-3 Intersection Pocket Lengths and Detector Locations

\begin{tabular}{|c|c|c|c|c|c|c|c|c|}
\hline \multicolumn{2}{|l|}{} & \multicolumn{2}{c|}{$\begin{array}{c}\text { POCKET } \\
\text { LENGTHS (FT) }\end{array}$} & \multicolumn{3}{c|}{$\begin{array}{c}\text { STOP BAR DETECTOR } \\
\text { LENGTHS (FT) }\end{array}$} & \multicolumn{2}{c|}{$\begin{array}{c}\text { RACEWAY } \\
\text { DETTORS } \\
\text { (FT) }\end{array}$} \\
\hline INT & MOVE & LEFT & RIGHT & LEFT & THRU & RIGHT & THRU & DIST \\
\hline 1 & NB & 100 & 240 & 36 & 36 & 36 & -- & -- \\
\hline 1 & SB & 665 & 240 & 36 & 36 & 36 & -- & -- \\
\hline 1 & EB & 250 & 70 & 36 & -- & -- & -- & -- \\
\hline 1 & WB & 200 & 112 & 36 & -- & -- & -- & -- \\
\hline 2 & NB & -- & -- & -- & -- & -- & -- & -- \\
\hline 2 & SB & 250 & -- & 36 & -- & -- & -- & -- \\
\hline 2 & WB & -- & -- & 36 & -- & 36 & -- & -- \\
\hline 3 & NB & -- & -- & -- & -- & -- & -- & -- \\
\hline 3 & SB & -- & -- & -- & -- & -- & -- & -- \\
\hline 3 & EB & -- & -- & 36 & -- & 36 & -- & -- \\
\hline 4 & NB & -- & -- & -- & -- & -- & -- & -- \\
\hline 4 & SB & -- & -- & -- & -- & -- & -- & -- \\
\hline 4 & WB & -- & -- & 36 & -- & 36 & -- & -- \\
\hline 5 & NB & 100 & -- & 36 & -- & -- & -- & -- \\
\hline 5 & SB & 60 & -- & 36 & -- & -- & -- & -- \\
\hline 5 & EB & -- & -- & 36 & 36 & -- & -- & -- \\
\hline 5 & WB & -- & -- & -- & 36 & -- & -- & -- \\
\hline
\end{tabular}


Table B-4 Controller Interface Device File (used for Hardware-in-the-Loop)

\begin{tabular}{|c|c|}
\hline COM [PortID] & 1, 4, R, 11, Prot \\
\hline 1 & $2,1, L, 8$, Prot \\
\hline CIDdef [CID, Base ID] & 2, 1, R, 8, Prot \\
\hline 1,1 & $2,2, \mathrm{~T}, 2$, Prot \\
\hline 2,3 & 2, 2, R, 2, Prot \\
\hline 3,5 & $2,3, L, 1, P P$ \\
\hline 4,7 & $2,3, \mathrm{~T}, 6$, Prot \\
\hline 5,9 & 3, 1, L, 4, Prot \\
\hline NodeAssign[Node, CID] & 3, 1, R, 4, Prot \\
\hline 1,1 & $3,2, \mathrm{~T}, 2$, Prot \\
\hline 2,2 & $3,3, T, 6$, Prot \\
\hline 3,3 & 4, 1, T, 6, Prot \\
\hline 4,4 & 4, 2, L, 8, Prot \\
\hline 5,5 & 4, 2, R, 8, Prot \\
\hline Phases [Node, Approach, & 4, 3, T, 2, Prot \\
\hline L/T/R, Phase\#, Pro/Per/PP] & $5,1, L, 5, P P$ \\
\hline $1,1, L, 5, P P$ & $5,1, T, 2$, Prot \\
\hline 1, 1, T, 2, Prot & 5, 1, R, 2, Prot \\
\hline 1, 1, R, 9, Prot & $5,2, L, 1, P P$ \\
\hline 1, 2, L, 7, PP & $5,2, T, 6$, Prot \\
\hline 1, 2, T, 4, Prot & 5, 2, R, 6, Prot \\
\hline 1, 2, R, 10, Prot & 5, 3, L, 4, Prot \\
\hline 1, 3, L, 3, PP & $5,3, T, 4$, Prot \\
\hline 1, 3, T, 8, Prot & 5, 3, R, 4, Prot \\
\hline 1, 3, R, 12, Prot & 5, 4, L, 3, Prot \\
\hline 1, 4, L, 1, PP & $5,4, T, 3$, Prot \\
\hline 1, 4, T, 6, Prot & 5,2 \\
\hline
\end{tabular}


Table B-5 Intersection Detector Configuration

\begin{tabular}{|c|c|c|c|c|c|c|}
\hline INT & $\begin{array}{l}\text { MOVE- } \\
\text { MENT }\end{array}$ & $\begin{array}{l}\text { DET } \\
\text { NUM }\end{array}$ & $\begin{array}{l}\text { LOCK I } \\
\text { N-LCK }\end{array}$ & $\begin{array}{c}\text { DELAY } \\
\text { (SEC) }\end{array}$ & $\begin{array}{c}\text { CALL } \\
\text { PHASE }\end{array}$ & DETECTOR TYPE \\
\hline 1 & NBLT & 105 & $\mathrm{~N}-\mathrm{LCK}$ & $\overline{--}$ & 5 & STANDARD (PRESENCE) \\
\hline 1 & NBLT & 115 & $\mathrm{~N}-\mathrm{LCK}$ & $\overline{--}$ & 5 & (PRESENCE) \\
\hline 1 & NBTH & 102 & $\mathrm{~N}-\mathrm{LCK}$ & -- & 2 & STANDARD (PRESENCE) \\
\hline 1 & NBTH & 112 & $\mathrm{~N}-\mathrm{LCK}$ & -- & 2 & (PRESENCE) \\
\hline 1 & NBTH & 110 & $\mathrm{~N}-\mathrm{LCK}$ & -- & 2 & STANDARD (PRESENCE) \\
\hline 1 & NBTH & 109 & N-LCK & -- & 2 & (PRESENCE) \\
\hline 1 & NBRT & 118 & $\mathrm{~N}-\mathrm{LCK}$ & 10 & 2 & STANDARD (PRESENCE) \\
\hline 1 & NBRT & 117 & $\mathrm{~N}-\mathrm{LCK}$ & -- & 2 & (PRESENCE) \\
\hline 1 & SBLT & 101 & N-LCK & -- & 1 & STANDARD (PRESENCE) \\
\hline 1 & SBLT & 111 & $\mathrm{~N}-\mathrm{LCK}$ & -- & 1 & (PRESENCE) \\
\hline 1 & SBTH & 106 & $\mathrm{~N}-\mathrm{LCK}$ & -- & 6 & STANDARD (PRESENCE) \\
\hline 1 & SBTH & 116 & $\mathrm{~N}-\mathrm{LCK}$ & -- & 6 & (PRESENCE) \\
\hline 1 & SBTH & 114 & $\mathrm{~N}-\mathrm{LCK}$ & -- & 6 & STANDARD (PRESENCE) \\
\hline 1 & SBTH & 113 & $\mathrm{~N}-\mathrm{LCK}$ & -- & 6 & (PRESENCE) \\
\hline 1 & SBRT & 120 & $\mathrm{~N}-\mathrm{LCK}$ & 10 & 6 & STANDARD (PRESENCE) \\
\hline 1 & SBRT & 119 & $\mathrm{~N}-\mathrm{LCK}$ & -- & 6 & (PRESENCE) \\
\hline 1 & EBLT & 107 & N-LCK & -- & 7 & STANDARD (PRESENCE) \\
\hline 1 & EBLT & 108 & $\mathrm{~N}-\mathrm{LCK}$ & -- & 7 & (PRESENCE) \\
\hline 1 & WBLT & 103 & $\mathrm{~N}-\mathrm{LCK}$ & -- & 3 & STANDARD (PRESENCE) \\
\hline 1 & WBLT & 104 & $\mathrm{~N}-\mathrm{LCK}$ & -- & 3 & (PRESENCE) \\
\hline 2 & SBLT & 201 & $\mathrm{~N}-\mathrm{LCK}$ & -- & 1 & STANDARD (PRESENCE) \\
\hline 2 & SBLT & 202 & N-LCK & -- & 1 & (PRESENCE) \\
\hline 2 & WBLT & 208 & $\mathrm{~N}-\mathrm{LCK}$ & -- & 8 & STANDARD (PRESENCE) \\
\hline 2 & WBLT & 209 & $\mathrm{~N}-\mathrm{LCK}$ & -- & 8 & (PRESENCE) \\
\hline 2 & WBRT & 216 & $\mathrm{~N}-\mathrm{LCK}$ & -- & 8 & STANDARD (PRESENCE) \\
\hline 2 & WBRT & 217 & $\mathrm{~N}-\mathrm{LCK}$ & -- & 8 & (PRESENCE) \\
\hline 3 & EBLT & 304 & $\mathrm{~N}-\mathrm{LCK}$ & -- & 4 & STANDARD (PRESENCE) \\
\hline 3 & EBLT & 305 & $\mathrm{~N}-\mathrm{LCK}$ & -- & 4 & (PRESENCE) \\
\hline 3 & EBRT & 312 & $\mathrm{~N}-\mathrm{LCK}$ & -- & 4 & STANDARD (PRESENCE) \\
\hline 3 & EBRT & 313 & $\mathrm{~N}-\mathrm{LCK}$ & -- & 4 & (PRESENCE) \\
\hline 4 & WBLT & 408 & $\mathrm{~N}-\mathrm{LCK}$ & $\overline{--}$ & 8 & STANDARD (PRESENCE) \\
\hline 4 & WBLT & 409 & $\mathrm{~N}-\mathrm{LCK}$ & -- & 8 & (PRESENCE) \\
\hline 4 & WBRT & 416 & $\mathrm{~N}-\mathrm{LCK}$ & -- & 8 & STANDARD (PRESENCE) \\
\hline 4 & WBRT & 417 & N-LCK & -- & 8 & (PRESENCE) \\
\hline 5 & NBLT & 505 & $\mathrm{~N}-\mathrm{LCK}$ & -- & 5 & STANDARD (PRESENCE) \\
\hline 5 & NBLT & 513 & N-LCK & -- & 5 & (PRESENCE) \\
\hline 5 & SBLT & 501 & $\mathrm{~N}-\mathrm{LCK}$ & -- & 1 & STANDARD (PRESENCE) \\
\hline 5 & SBLT & 509 & $\mathrm{~N}-\mathrm{LCK}$ & -- & 1 & (PRESENCE) \\
\hline 5 & EBLTH & 503 & $\mathrm{~N}-\mathrm{LCK}$ & -- & 3 & STANDARD (PRESENCE) \\
\hline 5 & EBLTH & 502 & $\mathrm{~N}-\mathrm{LCK}$ & -- & 3 & (PRESENCE) \\
\hline 5 & EBRT & 511 & $\mathrm{~N}-\mathrm{LCK}$ & -- & 3 & STANDARD (PRESENCE) \\
\hline 5 & EBRT & 506 & $\mathrm{~N}-\mathrm{LCK}$ & -- & 3 & (PRESENCE) \\
\hline 5 & WBTH & 504 & $\mathrm{~N}-\mathrm{LCK}$ & -- & 4 & STANDARD (PRESENCE) \\
\hline 5 & WBTH & 508 & $\mathrm{~N}-\mathrm{LCK}$ & -- & 4 & (PRESENCE) \\
\hline
\end{tabular}


Table B-6 General Phase Parameters for Intersection 1

\begin{tabular}{|c|c|c|c|c|c|c|c|c|c|}
\hline INT & DESCRIPTION (SEC) & $\mathbf{1}$ & $\mathbf{2}$ & $\mathbf{3}$ & $\mathbf{4}$ & $\mathbf{5}$ & $\mathbf{6}$ & $\mathbf{7}$ & $\mathbf{8}$ \\
\hline 1 & MINIMUM GREEN & 5 & 10 & 5 & 7 & 5 & 10 & 5 & 7 \\
\hline 1 & YELLOW CLEARANCE & 3.2 & 4.0 & 3.2 & 4.0 & 3.2 & 4.0 & 3.2 & 4.0 \\
\hline 1 & RED CLEARANCE & 2.0 & 1.5 & 2.0 & 1.5 & 2.0 & 1.5 & 2.0 & 1.5 \\
\hline 1 & PEDESTRIAN CLEARANCE & -- & 11 & -- & 11 & -- & 11 & -- & 11 \\
\hline 1 & PEDESTRIAN WALK & -- & 7 & -- & 7 & -- & 7 & -- & 7 \\
\hline 1 & MAX1 GREEN & 15 & 46 & 15 & 67 & 12 & 48 & 16 & 66 \\
\hline 1 & MAX2 GREEN & 15 & 46 & 15 & 67 & 12 & 48 & 16 & 66 \\
\hline 1 & MAX3 GREEN & 17 & 53 & 17 & 78 & 14 & 56 & 19 & 76 \\
\hline 1 & MAXIMUM EXTENSION & 1.0 & 3.5 & 1.0 & 5.5 & 1.0 & 4.0 & 1.5 & 5.0 \\
\hline 1 & VEHICLE EXTENSION & 1.4 & 5.0 & 1.4 & 5.0 & 1.4 & 5.0 & 1.4 & 5.0 \\
\hline 1 & TIME BEFORE REDUCTION & -- & 15 & -- & 22 & -- & 18 & -- & 25 \\
\hline 1 & TIME TO REDUCE & -- & 37 & -- & 54 & -- & 45 & -- & 61 \\
\hline 1 & MINIMUM GAP TIME & -- & 3.0 & -- & 3.0 & -- & 3.0 & -- & 3.0 \\
\hline 1 & SEC/ACTUATION & -- & -- & -- & -- & -- & -- & -- & -- \\
\hline 1 & MAXIMUM INITIAL & -- & -- & -- & -- & -- & -- & -- & -- \\
\hline
\end{tabular}

Table B-7 General Phase Parameters for Intersection 2

\begin{tabular}{|c|c|c|c|c|c|c|c|c|c|}
\hline INT & DESCRIPTION (SEC) & $\mathbf{1}$ & $\mathbf{2}$ & $\mathbf{3}$ & $\mathbf{4}$ & $\mathbf{5}$ & $\mathbf{6}$ & $\mathbf{7}$ & $\mathbf{8}$ \\
\hline 2 & MINIMUM GREEN & 5 & 10 & -- & -- & -- & 10 & -- & 7 \\
\hline 2 & YELLOW CLEARANCE & 3.0 & 4.0 & -- & -- & -- & 4.0 & -- & 3.5 \\
\hline 2 & RED CLEARANCE & 1.0 & 1.0 & -- & -- & -- & 1.0 & -- & 1.5 \\
\hline 2 & PEDESTRIAN CLEARANCE & -- & 3 & -- & -- & -- & -- & -- & 9 \\
\hline 2 & PEDESTRIAN WALK & -- & 7 & -- & -- & -- & -- & -- & 7 \\
\hline 2 & MAX1 GREEN & 18 & 34 & -- & -- & -- & 52 & -- & 26 \\
\hline 2 & MAX2 GREEN & 18 & 34 & -- & -- & -- & 52 & -- & 26 \\
\hline 2 & MAX3 GREEN & 21 & 39 & -- & -- & -- & 60 & -- & 30 \\
\hline 2 & MAXIMUM EXTENSION & 1.5 & 2.5 & -- & -- & -- & 4.0 & -- & 2.0 \\
\hline 2 & VEHICLE EXTENSION & 1.4 & -- & -- & -- & -- & -- & -- & 1.6 \\
\hline 2 & TIME BEFORE REDUCTION & -- & -- & -- & -- & -- & -- & -- & -- \\
\hline 2 & TIME TO REDUCE & -- & -- & -- & -- & -- & -- & -- & -- \\
\hline 2 & MINIMUM GAP TIME & -- & -- & -- & -- & -- & -- & -- & -- \\
\hline 2 & SEC/ACTUATION & -- & -- & -- & -- & -- & -- & -- & -- \\
\hline 2 & MAXIMUM INITIAL & -- & -- & -- & -- & -- & -- & -- & -- \\
\hline
\end{tabular}


Table B-8 General Phase Parameters for Intersection 3

\begin{tabular}{|c|c|c|c|c|c|c|c|c|c|}
\hline INT & DESCRIPTION (SEC) & $\mathbf{1}$ & $\mathbf{2}$ & $\mathbf{3}$ & $\mathbf{4}$ & $\mathbf{5}$ & $\mathbf{6}$ & $\mathbf{7}$ & $\mathbf{8}$ \\
\hline 3 & MINIMUM GREEN & -- & 10 & -- & 7 & -- & 10 & -- & -- \\
\hline 3 & YELLOW CLEARANCE & -- & 4.0 & -- & 3.5 & -- & 4.0 & -- & -- \\
\hline 3 & RED CLEARANCE & -- & 1.0 & -- & 1.5 & -- & 1.0 & -- & -- \\
\hline 3 & PEDESTRIAN CLEARANCE & -- & -- & -- & 9 & -- & 0 & -- & -- \\
\hline 3 & PEDESTRIAN WALK & -- & -- & -- & 7 & -- & 7 & -- & -- \\
\hline 3 & MAX1 GREEN & -- & 43 & -- & 35 & -- & 43 & -- & -- \\
\hline 3 & MAX2 GREEN & -- & 43 & -- & 35 & -- & 43 & -- & -- \\
\hline 3 & MAX3 GREEN & -- & 50 & -- & 40 & -- & 50 & -- & -- \\
\hline 3 & MAXIMUM EXTENSION & -- & 3.5 & -- & 2.5 & -- & 3.5 & -- & -- \\
\hline 3 & VEHICLE EXTENSION & -- & -- & -- & 1.6 & -- & -- & -- & -- \\
\hline 3 & TIME BEFORE REDUCTION & -- & -- & -- & -- & -- & -- & -- & -- \\
\hline 3 & TIME TO REDUCE & -- & -- & -- & -- & -- & -- & -- & -- \\
\hline 3 & MINIMUM GAP TIME & -- & -- & -- & -- & -- & -- & -- & -- \\
\hline 3 & SEC/ACTUATION & -- & -- & -- & -- & -- & -- & -- & -- \\
\hline 3 & MAXIMUM INITIAL & -- & -- & -- & -- & -- & -- & -- & -- \\
\hline
\end{tabular}

Table B-9 General Phase Parameters for Intersection 4

\begin{tabular}{|c|c|c|c|c|c|c|c|c|c|}
\hline INT & DESCRIPTION (SEC) & $\mathbf{1}$ & $\mathbf{2}$ & $\mathbf{3}$ & $\mathbf{4}$ & $\mathbf{5}$ & $\mathbf{6}$ & $\mathbf{7}$ & $\mathbf{8}$ \\
\hline 4 & MINIMUM GREEN & -- & 10 & -- & -- & -- & 10 & -- & 7 \\
\hline 4 & YELLOW CLEARANCE & -- & 4.0 & -- & -- & -- & 4.0 & -- & 3.5 \\
\hline 4 & RED CLEARANCE & -- & 1.0 & -- & -- & -- & 1.0 & -- & 1.5 \\
\hline 4 & PEDESTRIAN CLEARANCE & -- & 0 & -- & -- & -- & -- & -- & 9 \\
\hline 4 & PEDESTRIAN WALK & -- & 7 & -- & -- & -- & -- & -- & 7 \\
\hline 4 & MAX1 GREEN & -- & 35 & -- & -- & -- & 35 & -- & 43 \\
\hline 4 & MAX2 GREEN & -- & 35 & -- & -- & -- & 35 & -- & 43 \\
\hline 4 & MAX3 GREEN & -- & 40 & -- & -- & -- & 40 & -- & 50 \\
\hline 4 & MAXIMUM EXTENSION & -- & 2.5 & -- & -- & -- & 2.5 & -- & 3.5 \\
\hline 4 & VEHICLE EXTENSION & -- & -- & -- & -- & -- & -- & -- & 1.6 \\
\hline 4 & TIME BEFORE REDUCTION & -- & -- & -- & -- & -- & -- & -- & -- \\
\hline 4 & TIME TO REDUCE & -- & -- & -- & -- & -- & -- & -- & -- \\
\hline 4 & MINIMUM GAP TIME & -- & -- & -- & -- & -- & -- & -- & -- \\
\hline 4 & SEC/ACTUATION & -- & -- & -- & -- & -- & -- & -- & -- \\
\hline 4 & MAXIMUM INITIAL & -- & -- & -- & -- & -- & -- & -- & -- \\
\hline
\end{tabular}


Table B-10 General Phase Parameters for Intersection 5

\begin{tabular}{|c|c|c|c|c|c|c|c|c|c|}
\hline INT & DESCRIPTION (SEC) & $\mathbf{1}$ & $\mathbf{2}$ & $\mathbf{3}$ & $\mathbf{4}$ & $\mathbf{5}$ & $\mathbf{6}$ & $\mathbf{7}$ & $\mathbf{8}$ \\
\hline 5 & MINIMUM GREEN & 5 & 10 & 7 & 7 & 5 & 10 & -- & -- \\
\hline 5 & YELLOW CLEARANCE & 3.0 & 4.0 & 3.5 & 3.5 & 3.0 & 4.0 & -- & -- \\
\hline 5 & RED CLEARANCE & 1.5 & 1.0 & 1.5 & 1.5 & 1.5 & 1.0 & -- & -- \\
\hline 5 & PEDESTRIAN CLEARANCE & -- & 0 & 9 & 9 & -- & 6 & -- & -- \\
\hline 5 & PEDESTRIAN WALK & -- & 7 & 7 & 7 & -- & 7 & -- & -- \\
\hline 5 & MAX1 GREEN & 13 & 68 & 42 & 34 & 23 & 57 & -- & -- \\
\hline 5 & MAX2 GREEN & 13 & 68 & 42 & 34 & 23 & 57 & -- & -- \\
\hline 5 & MAX3 GREEN & 15 & 78 & 48 & 39 & 27 & 66 & -- & -- \\
\hline 5 & MAXIMUM EXTENSION & 1.0 & 5.0 & 3.0 & 2.5 & 2.0 & 4.5 & -- & -- \\
\hline 5 & VEHICLE EXTENSION & 1.4 & -- & 1.6 & 1.6 & 1.4 & -- & -- & -- \\
\hline 5 & TIME BEFORE REDUCTION & -- & -- & -- & -- & -- & -- & -- & -- \\
\hline 5 & TIME TO REDUCE & -- & -- & -- & -- & -- & -- & -- & -- \\
\hline 5 & MINIMUM GAP TIME & -- & -- & -- & -- & -- & -- & -- & -- \\
\hline 5 & SEC/ACTUATION & -- & -- & -- & -- & -- & -- & -- & -- \\
\hline 5 & MAXIMUM INITIAL & -- & -- & -- & -- & -- & -- & -- & -- \\
\hline
\end{tabular}

Table B-11 Coordination Plans and Splits

\begin{tabular}{|c|c|c|c|c|c|c|c|c|c|c|c|}
\hline INT & COS & CYC & OFF & $\mathbf{1}$ & $\mathbf{2}$ & $\mathbf{3}$ & $\mathbf{4}$ & $\mathbf{5}$ & $\mathbf{6}$ & $\mathbf{7}$ & $\mathbf{8}$ \\
\hline 1 & 111 & 90 & 0 & 11 & 32 & 11 & 46 & 11 & 32 & 11 & 46 \\
\hline 2 & 111 & 90 & 22 & 29 & 43 & -- & -- & -- & 72 & -- & 28 \\
\hline 3 & 111 & 90 & 44 & -- & 59 & -- & 41 & -- & 59 & -- & -- \\
\hline 4 & 111 & 45 & 13 & -- & 49 & -- & -- & -- & 49 & -- & 51 \\
\hline 5 & 111 & 90 & 63 & 11 & 31 & 29 & 29 & 16 & 27 & -- & -- \\
\hline 1 & 222 & 100 & 0 & 10 & 30 & 10 & 50 & 10 & 30 & 12 & 48 \\
\hline 2 & 222 & 100 & 24 & 28 & 44 & -- & -- & -- & 72 & -- & 28 \\
\hline 3 & 222 & 100 & 43 & -- & 60 & -- & 40 & -- & 60 & -- & -- \\
\hline 4 & 222 & 50 & 8 & -- & 46 & -- & -- & -- & 46 & -- & 54 \\
\hline 5 & 222 & 100 & 68 & 10 & 38 & 26 & 26 & 18 & 30 & -- & -- \\
\hline 1 & 333 & 120 & 0 & 8 & 31 & 8 & 53 & 8 & 31 & 10 & 51 \\
\hline 2 & 333 & 60 & 14 & 23 & 43 & -- & -- & -- & 67 & -- & 33 \\
\hline 3 & 333 & 60 & 52 & -- & 55 & -- & 45 & -- & 55 & -- & -- \\
\hline 4 & 333 & 60 & 39 & -- & 45 & -- & -- & -- & 45 & -- & 55 \\
\hline 5 & 333 & 120 & 33 & 8 & 43 & 27 & 22 & 15 & 37 & -- & -- \\
\hline
\end{tabular}




\section{List of References}

Econolite Control Products, Inc., "Advanced System Controller ASC/2 Programming Manual, 1996.

Econolite Control Products, Inc., "ASC/2M-1000 Zone Master Programming Manual”, 1993.

Greenshields, B.D. "A Study in Highway Capacity." Highway Research Board, Volume 14, Page 458, 1935.

Kell, J.H. and I.J. Fullerton. "Manual of Traffic Signal Design," Institute of Transportation Engineers, Prentice Hall, 1991.

Manual on Uniform Traffic Control Devices, Federal Highway Administration, U.S. Department of Transportation, 2000.

National Electrical Manufacturers Association, Standards Publication No. TS 1, Washington, DC, 1989.

National Electrical Manufacturers Association, Standards Publication No. TS 2, Washington, DC, 1992.

Peek Traffic - Transyt, “3000 TS1/TS2 Controller Operating Manual, Revision 5.0”, 1996.

Peek Traffic - Transyt, “M3000 Series On-street Master Operating Manual, Revision 2.1”, 1996.

"Transportation Infrastructure, Benefits of Traffic Control Signal Systems Are Not Being Fully Realized," GAO/RCED-94-105, Report to the Chairman, Committee on Energy and Commerce, House of Representatives, United States General Accounting Office, Washington, DC, March 1994. 